\title{
Palaearctic species of Charipinae (Hymenoptera, Figitidae): two new species, synthesis and identification key
}

\author{
Mar FERRER-SUAY ${ }^{1, *}$, Jesús SELFA $^{2}$ \& Juli PUJADE-VILLAR ${ }^{3}$ \\ ${ }^{1,2}$ Universitat de València, Facultat de Ciències Biològiques, Departament de Zoologia. Campus de \\ Burjassot-Paterna, Dr. Moliner 50, 46100 Burjassot (València), Spain. \\ ${ }^{3}$ Universitat de Barcelona, Facultat de Biologia, Departament de Biologia Animal, Avda. Diagonal \\ 645, 08028-Barcelona, Spain. \\ ${ }^{*}$ Corresponding author: mar.ferrer.suay@gmail.com \\ ${ }^{2}$ Email: jesus.selfa@uv.es \\ ${ }^{3}$ Email: jpujade@ub.edu

\begin{abstract}
${ }^{1}$ urn:lsid:zoobank.org:author:B7059757-51CD-45DD-A182-C64FA5EF63C8
${ }^{2}$ urn:lsid:zoobank.org:author:C01B4FA6-6C5C-4DDF-A114-2B06D8FE4D20

${ }^{3}$ urn:lsid:zoobank.org:author:94C497E0-C6A1-48BD-819D-FE5A8036BECD
\end{abstract}

\begin{abstract}
The Charipinae Dalla Torre \& Kieffer, 1910 present in the Palaearctic region are revised; 2410 specimens have been identified, belonging to 75 species: 52 to Alloxysta, one to Apocharips, six to Dilyta and 16 to Phaenoglyphis. For 33 species, new country-level distribution records are provided. Two new species are here described: Alloxysta palearctica Ferrer-Suay \& Pujade-Villar sp. nov. and Alloxysta pascuali Ferrer-Suay sp. nov. A diagnosis for these species is included and their diagnostic features are shown in different figures. A key to identify all the species of Charipinae in the Palaearctic region is also given.
\end{abstract}

Keywords. Charipinae, Palaearctic, key, Alloxysta, Apocharips, Dilyta, Phaenoglyphis.

Ferrer-Suay M., Selfa J. \& Pujade-Villar J. 2018. Palaearctic species of Charipinae (Hymenoptera, Figitidae): two new species, synthesis and identification key. European Journal of Taxonomy 427: 1-110. https://doi.org/10.5852/ejt.2018.427

\section{Introduction}

Charipinae Dalla Torre \& Kieffer, 1910 is one subfamily of the family Figitidae Thomson, 1862 (Hymenoptera Linnaeus, 1758: Cynipoidea Billberg, 1820). This subfamily is economically very important because its members act as secondary parasitoids of aphids via Aphidiinae Haliday, 1833 (Hymenoptera: Braconidae Nees, 1811) and Aphelininae Thomson, 1876 (Hymenoptera: Aphelinidae Thomson, 1876) and of psyllids via Encyrtidae Walker, 1837 (Hymenoptera: Chalcidoidea Latreille, 1817) (Menke \& Evenhuis 1991). The efficiency of primary parasitoids on aphid biological control management is generally impaired by the activity of different hyperparasitoids (Sullivan 1987). The species of Charipinae are considered to be the most common and specialized aphid hyperparasitoids (Carver 1992). 
In this subfamily, eight genera are distinguished: Alloxysta Förster, 1869 (cosmopolitan), Apocharips Fergusson, 1986 (Eastern Palaearctic and Neotropics), Dilapothor Paretas-Martínez \& Pujade-Villar, 2006 (Australia), Dilyta Förster, 1869 (cosmopolitan, except Australia), Lobopterocharips Paretas-Martínez \& Pujade-Villar, 2007 (Nepal), Lytoxysta Kieffer, 1909 (North America), Phaenoglyphis Förster, 1869 (cosmopolitan) and Thoreauana Girault, 1930 (Australia). Charipinae is a widely distributed group of insects that have diversified along with their primary host parasitoids and secondary hosts.

Morphologically, the Charipinae are characterized by a very small $(0.8-2.0 \mathrm{~mm})$, shiny and smooth body, filiform antenna and venation of the fore wing that is reduced to only a small radial cell. These features make identification at species level a very difficult task and thus taxonomy has been problematic. Until now, few diagnostic characters useful for species identification have been established. As part of a worldwide revision of Charipinae, those from the Nearctic, Neotropical, African, Asian and Australian regions have been studied; in this work we focus on the species present in the Palaearctic region. This way, a complete worldwide revision will be established.

Types from the following collections have been examined previously: the Vladimir I. Belizin collection deposited at the Zoological Institute of the Russian Academy of Sciences, Saint Petersburg, Russia (Ferrer-Suay et al. 2012d); the Mihail A. Ionescu collection deposited at the "Grigore Antipa" National Museum of Natural History, Bucharest, Romania (Ferrer-Suay et al. 2012e); the Carl G. Thomson and Johan W. Zetterstedt collections deposited at the Lund Museum of Zoology, Sweden (Ferrer-Suay et al. 2013d); the Theodor Hartig collection deposited at the Zoologische Staatssammlung Museum, Munich, Germany (Ferrer-Suay et al. 2015b); the Wolter Hellén collection deposited at the Finnish Museum of Natural History, Helsinki, Finland (Ferrer-Suay et al. 2014c); the John Curtis collection deposited at the National Museum of Victoria, Melbourne, Australia (Ferrer-Suay et al. 2013e); the Fred G. Andrews, William H. Ashmead and Charles F. Baker collections deposited at the United States National Museum of Natural History (Smithsonian Institution), Washington DC, USA and at the Canadian National Collection of Insects, Ottawa, Canada (Ferrer-Suay et al. 2013h); and the Peter Cameron and Nigel D.M. Fergusson collections deposited in the Natural History Museum, London (Ferrer-Suay et al. 2013c). We have examined the fauna of the Oriental (Ferrer-Suay et al. 2013i), Australian (Ferrer-Suay et al. 2014d), African (Ferrer-Suay et al. 2013j), Neotropical (Ferrer-Suay et al. 2013k) and Nearctic (Ferrer-Suay et al. 2014a) regions.

Several works have focused on the Charipinae of different areas within the Palaearctic, but, until now, none has focused on this region as a whole. Fergusson (1986) focused on the Charipinae of Great Britain; at different levels Thomson (1862) did the same for Sweden; Hellén (1963) for Finland; Hartig (1840, 1841) for Germany; Ionescu (1959) for Romania; and Belizin (1962, 1966, 1968, 1973) for Russia. Kieffer $(1900,1902 a, 1902 b, 1909)$ described species from different localities within the Palaearctic and European species were included in Dalla Torre \& Kieffer's catalogue. Finally, a catalogue of the world's Charipinae species has recently been published (Ferrer-Suay et al. 2012a); nevertheless, in the last few years the knowledge of Charipinae has been substantially modified, which justifies this study.

The authors of this work have already revised material of Charipinae from different Palaearctic countries: Andorra (Ferrer-Suay et al. 2011), Catalonia (Ferrer-Suay et al. 2012c), Iran (Ferrer-Suay et al. 2013a), the Balkan Peninsula (Ferrer-Suay et al. 2013b), Italy (Ferrer-Suay et al. 2014b) and south-eastern France (Ferrer-Suay et al. 2015a). With this revision, we intend to group all this information as well as improve it with the study of new material.

After studying the material of Charipinae present at several institutions (the Natural History Museum London, Canadian National Collection of Insects and the United States National Museum of Natural History - Smithsonian Institution) and taking into account the previous works focused on this region, we 
found that only four of the eight genera of Charipinae are present in the Palaearctic region: Alloxysta with 52 species, Apocharips with only one species, Dilyta with five and Phaenoglyphis with 16. Diagnoses of all species are given, with images. New country-level distribution records are presented for 33 species in this study and two new species are here described: Alloxysta palearctica Ferrer-Suay \& Pujade-Villar sp. nov. and Alloxysta pascuali Ferrer-Suay sp. nov.

\section{Material and methods}

Specimens were studied using a stereo microscope (NIKON SMZ-1) and environmental scanning electron microscope (FEI Quanta 200 ESEM) belonging to the scientific technical services of the University of Barcelona. The field-emission gun environmental scanning electron microscope was used for high-resolution imaging without gold-coating of the specimens.

Material studied is deposited in the following institutions:

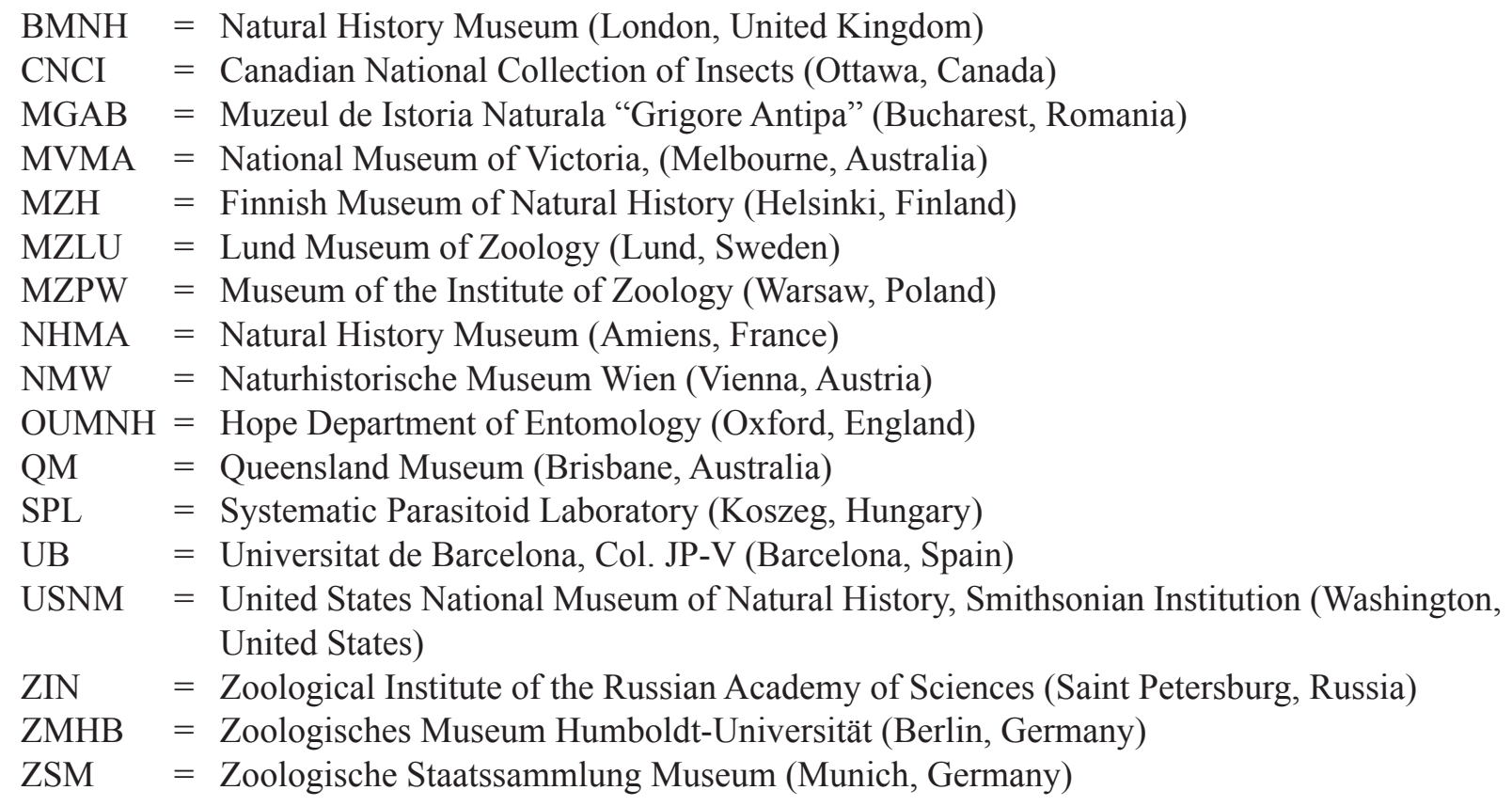

Identification of these taxa has been very difficult and confused in the past. For this reason, country records are separated in uncertain and certain records: country records that have been confirmed by the authors themselves after having observed specimens (including type specimens and type specimens of synonyms) are separated from those that are merely cited from literature and thus in practice uncertain.

Morphological terms used are taken from Paretas-Martínez et al. (2007a). The term 'club shape' is used in this manuscript to refer to the part of the antenna where the flagellomeres are wider than the previous one, and this expansion, more or less clear, continues until the end of the antenna. Usually, but not always, the beginning of the 'club shape' appears together with 'rhinaria' (placoidea sensilla) on the antenna.

Measurements and abbreviations include F1-F12, for the first and subsequent flagellomeres. The width of the fore wing radial cell is measured from the margin of the wing to the beginning of vein Rs. The transfacial line is measured as the distance between the inner margins of the compound eyes, measured across the face through the antennal sockets, divided by the height of the eye. The malar space is measured by the distance from the lower part of the gena from the mouthparts to the ventral margin of the compound eye, divided by the height of the eye. Females and males have the same characters, except where indicated. 
Figure 1 shows the types of mesopleuron and metasoma present in Charipinae from the Palaearctic region. For a better comparison, the Figures have been grouped in the following order: antenna of species of Alloxysta in Figs 2-3, the radial cell of species of Alloxysta in Figs 4-5, antenna of Apocharips and Phaenoglyphis in Fig. 6, mesoscutum in Fig. 7, different types of propodeum in Fig. 8, different types of pronotum in Fig. 9 and different types of fore wing in Fig. 10. Figures 11-12 show the characteristic features of Alloxysta palearctica Ferrer-Suay \& Pujade-Villar sp. nov. and Alloxysta pascuali FerrerSuay sp. nov., respectively.

Labels are literally quoted between straight brackets in the 'Material examined' sections.

\section{Results}

Class Hexapoda Blainville, 1816

Order Hymenoptera Linnaeus, 1758

Suborder Apocrita Latreille, 1810

Superfamily Cynipoidea Billberg, 1820

Family Figitidae Thomson, 1862

Subfamily Charipinae Dalla Torre \& Kieffer, 1910

\section{Key of Palaearctic Charipinae species}

1. Metasoma with a single tergal plate, or if two, then basal tergite much shorter than second along middorsal line (Fig. 1.4)

- Metasoma with two large visible terga, subequal in length along middorsal line, but basal tergite $1 / 4-1 / 3$ smaller than second in lateral view (Fig. 1.3)

2. Basal tergite much shorter than second along middorsal line Radial cell short, 1.2 times as long as wide, with R1 and Rs parallel

Apocharips Fergusson, 1986 Apocharips trapezoidea (Hartig, 1841)

- Metasoma with a single tergal plate 3 (Dilyta Förster, 1869)

3. Metasoma without punctures Dilyta aleevae Pujade-Villar \& Paretas-Martínez, 2011

- Metasoma with a punctate area on distal part

4. Male: F1 very long, wide and arched, much longer than pedicel (almost double) and longer than F2 and $\mathrm{F} 3$ together; $\mathrm{F} 2$ slightly shorter or subequal to F3; F4 longer than F2 and F3; F4-F12 wider than previous segments; antenna slightly clavate from F4; sensilla beginning on F4 (Fig. 6.5). Female unknown ...

Dilyta sinica Ferrer-Suay \& Paretas-Martínez, 2011

- Male, when known, with different features than given above ..................................5

5. Female: F1 very long, thin, almost twice as long as pedicel, longer than F2-F5 and nearly as long as F2+F3+F4 combined (Fig. 6.2). Male unknown

Dilyta japonica Paretas-Martínez \& Ferrer-Suay, 2011

- F1 subequal to pedicel and shorter or subequal to F2 and F3 combined .........................6

6. Female: F1 slightly shorter or subequal than pedicel; F2 subequal to F3; F4 slightly shorter than F1, but longer than F2 or F3; F1 subequal to F5; F6 longer than F5 (Fig. 6.6). Male: F1 slightly longer than pedicel; F2 or F3 each shorter than F1; F1 subequal to F4; F4-F12 wider than previous flagellomeres; antenna slightly clavate from F4; sensilla beginning on F4

Dilyta subclavata Förster, 1869 
- Female: F1 subequal to pedicel or slightly longer; F2 shorter than F3; F3 shorter than F4; F4 shorter than F5; F1 subequal to F5 (Fig. 6.3). Male: F1 subequal to pedicel; F2 shorter than F1 or F3; F3 subequal to F1; F3-F12 wider than previous flagellomeres; antenna slightly clavate from F3; sensilla beginning on F3 ......Dilyta longinqua Paretas-Martínez \& Pujade-Villar, 2011

7. Lower part of mesopleuron with horizontal sulcus (Fig. 1.1) ...8 (Phaenoglyphis Förster, 1869)

- Mesopleuron lacks horizontal sulcus (Fig. 1.2) ......................23 (Alloxysta Förster, 1869)

8. Notauli present, at least in the posterior half of mesoscutum and/or scutum sculptured ......9

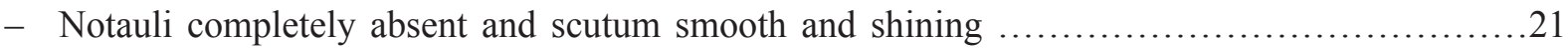

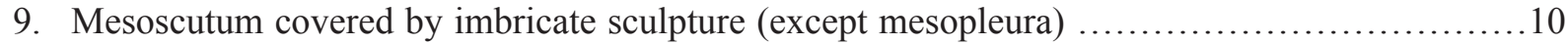

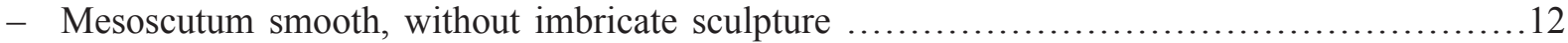

10. Mesoscutum mostly smooth, with a few wrinkles on the distal side of the notauli; rhinaria and club shape begin on the last three quarters of F1 (Fig. 6.18). Male antenna: rhinaria and club shape begin on F1; F1 curved; F1 longer than pedicel and F2; F2-F11 subequal ......................

.Phaenoglyphis ruficornis (Förster, 1869)
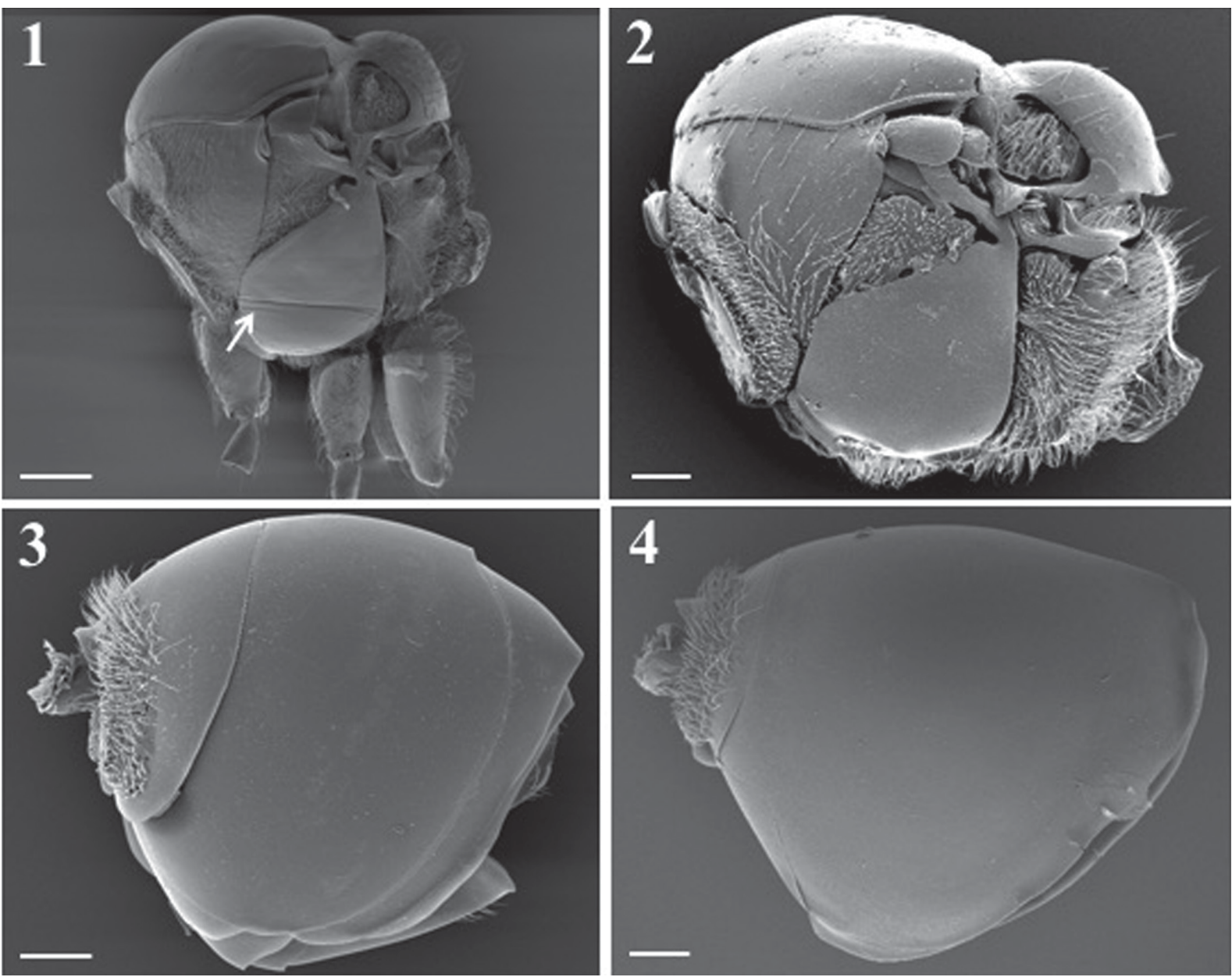

Fig. 1. Types of mesosoma and metasoma of the Charipinae Dalla Torre \& Kieffer, 1910. 1. Mesosoma of Phaenoglyphis spp. 2. Mesosoma of Alloxysta spp. 3. Metasoma of Alloxysta spp. 4. Metasoma of Apocharips spp. Scale bars: $50 \mu \mathrm{m}$. 
- With distinctive imbricate sculpture on all surfaces; rhinaria and club shape begin on different

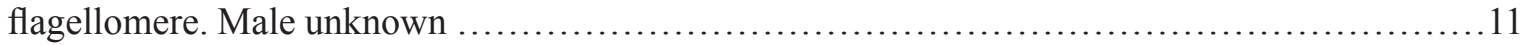

11. Rhinaria and club shape begin on F1 (Fig. 6.17); thick pronotal carinae not reaching the mesoscutum; mesoscutum enterely covered by many setae; notauli present, only indicated anteriorly and well-marked posteriorly; scutellar foveae open posteriorly (Fig. 7.11); central part of metascutellum smooth, only with a central carina; fore wing with long marginal setae. Male unknown

Phaenoglyphis pubicollis (Thomson, 1877)

- Rhinaria and club shape begin on F4 (Fig. 6.8); long pronotal carinae reaching the mesoscutum; mesoscutum with few scattered setae; notauli only indicated; scutellar foveae completely defined and with a transverse posterior carina inside (Fig. 7.2); central part of metascutellum with imbricate sculpture; fore wing without marginal setae (Fig. 10.1). Male unknown

Phaenoglyphis evenhuisi Pujade-Villar \& Paretas-Martínez, 2006

12. Head, mesosoma and metasoma yellowish brown; notauli deeply excavate, rounded scutellar foveae, but with interior side straight (Fig. 7.16). Male unknown

Phaenoglyphis xanthochroa Förster, 1869

- Head, mesosoma and metasoma dark brown; notauli present but not deeply excatave; different shape

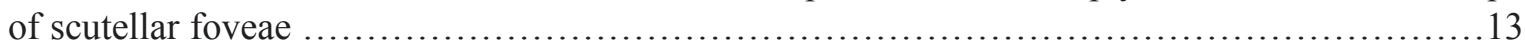

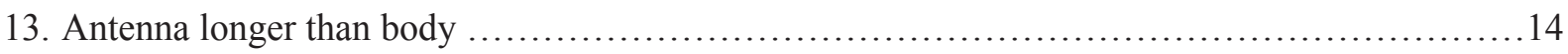

- Antenna subequal to or shorter than body ................................................ 18

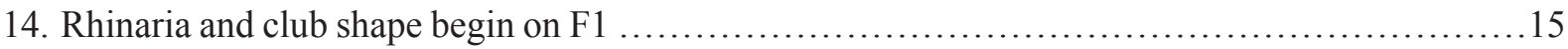

- Rhinaria and club shape begin on other flagellomeres ................................... 17

15. Rhinaria and club shape begin at the proximal end of F1; F2 subequal to F3 and F3 shorter than F4 (Fig. 6.13); Rs slightly curved. Male unknown ...Phaenoglyphis longicornis (Hartig, 1840)

- Rhinaria and club shape begin on the last three-quarters of F1; different proportions between flagellomeres; Rs straight .............................................................. 16

16. Last flagellomere 2.5 times as long as wide (Fig. 6.20); scutellar foveae with straight sides and open anteriorly and posteriorly (Fig. 7.14); radial cell 2.4 times as long as wide. Male unknown ...

Phaenoglyphis stricta (Thomson, 1877)

- Last flagellomere 4.3 times as long as wide (Fig. 6.11); rounded scutellar foveae, slightly open posteriorly (Fig. 7.5); radial cell 2.9 times as long as wide. Male unknown

Phaenoglyphis insperatus Belizin, 1973

17. Rhinaria and club shape not beginning on the same flagellomere, rhinaria begin on F1 and club shape begins on F3, F1 longer than pedicel (Fig. 6.9). Male antenna: club shape begins on F3, rhinaria on F5, F2 subequal to F3, F3 shorter than F4

Phaenoglyphis fuscicornis (Thomson, 1877)

- Rhinaria and club shape both begin on F3, F1 shorter than pedicel (Fig. 6.10). Male antenna: rhinaria and club shape begin on F3, F2 shorter than F3, F3 subequal to F4

Phaenoglyphis heterocera (Hartig, 1841)

18. F1 longer than pedicel, 1.1 times as long as pedicel and longer than F2; F2 shorter than F3; F3F4 subequal in length (Fig. 6.19); mesoscutum with a line of setae next to each notaulus; notauli weakly present (Fig. 7.13); scutellar foveae completely defined and with two lines anteriorly; propodeum with two short and straight carinae reaching the base independently. Male unknown 
- F1 subequal to or shorter than pedicel; different combination of features

19. Beginning of rhinaria in F2; F1 longer than F2; F2-F4 subequal in length; radial cell 2.7 times as long as wide

Phaenoglyphis proximus Belizin, 1966

- Beginning of rhinaria in F3; different proportions between flagellomeres; different size of radial cell

20. Female: F1 subequal to F2; F2-F4 subequal in length (Fig. 6.14); scutellar foveae rounded and separated by a thin carina (Fig. 7.8); with abundant setae on apex of scutellum; propodeum with narrow carinae (sometimes difficult to see). Male antenna: rhinaria and club shape begin on F3; F1 not curved, longer than pedicel and F2; F2 shorter than F3; F3-F4 subequal

Phaenoglyphis moldavica Ionescu, 1969

- Female: F1 longer than F2, but shorter than F3; F3 subequal to F4 (Fig. 6.7); scutellar foveae completely defined (Fig. 7.1). Male unknown ......Phaenoglyphis abbreviata (Thomson, 1877)

21. Scutellar foveae not present Phaenoglyphis nigripes (Thomson, 1877)

- Scutellar foveae present, sometimes superficially .22

22. Radial cell partially open along anterior margin; F1 and F2 subequal; F1 curved in males (Fig. 6.21)

Phaenoglyphis villosa (Hartig, 1841)

- Radial cell closed; F1 longer than F2 (Fig. 6.12). Male unknown

Phaenoglyphis insularis (Belizin, 1973)

23. Brachypterous species

- Fully winged species, wing usually longer than mesosoma and metasoma .......................31

24. Fore wing reaching the end of the metasoma; visible radial cell

- Fore wing reaching the beginning of the metasoma; without radial cell visible

25. Completely open radial cell (Fig. 4.22)

Alloxysta marshalliana (Kieffer, 1900)

- Closed radial cell

26. Pronotal carinae absent

Alloxysta glebaria Hellén, 1963

- Pronotal carinae present

27. Propodeal carinae absent

Alloxysta pseudoconsobrina Ferrer-Suay, 2017

- Propodeal carinae present Alloxysta curta Ferrer-Suay, 2017

28. Pronotal carinae present; propodeal carinae absent. Male always and female sometimes brachypterous Alloxysta halterata (Thomson, 1862)

- Pronotal carinae absent; propodeal carinae absent or present

29. Propodeal carinae well defined, joining posteriorly. Male: F1 shorter than pedicel (Fig. 2.8). Female unknown

Alloxysta brachyptera (Hartig, 1840)

- Propodeal carinae absent. F1 shorter or longer than pedicel

30. Fore wing reaching the beginning of metasoma. Female: F1 longer than pedicel and F2; F2 subequal to F3 (Fig. 3.1). Male: F1 subequal or slightly longer than pedicel, F1-F4 subequal in length Alloxysta pedestris (Curtis, 1838)

- Fore wing very short, practically absent. Female: F1 shorter than pedicel, F1 longer than F2, F2 subequal to F3 (Fig. 2.3). Male unknown Alloxysta apteroidea Hellén, 1963 


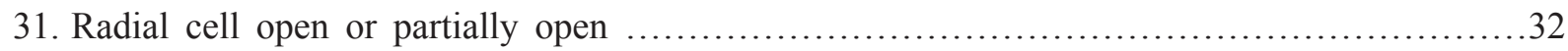

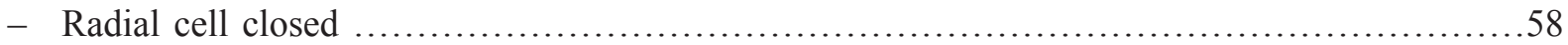

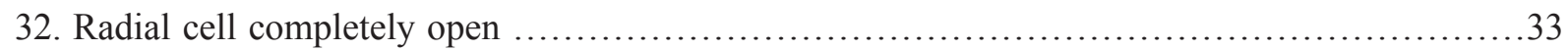

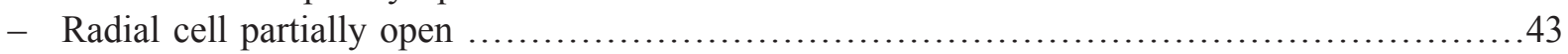

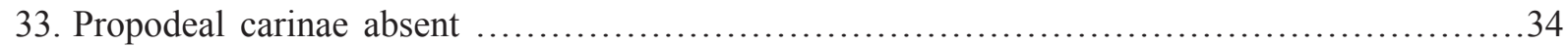

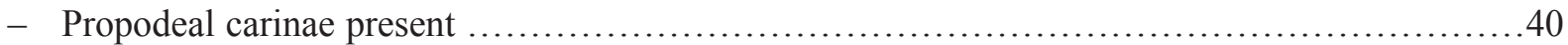

34. Male: F1 and F2 clearly bumped; F1 subequal to F2; F2 longer than F3, but shorter than F4 (Fig. 11.2-3); radial cell 2.3 times as long as wide (Fig. 11.4). Female unknown ...... Alloxysta palearctica Ferrer-Suay \& Pujade-Villar sp. nov.

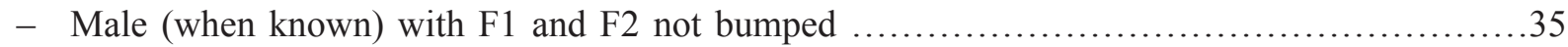

35. F2 longer than F1 and F3 (Fig. 3.6); radial cell 2.3 times as long as wide (Fig. 5.10)

Alloxysta proxima Belizin, 1962

- F2 shorter than or subequal to F1; radial cell longer or shorter than 2.3 times as long as wide ...36

36. Female: F2 subequal to F1; F2 longer than F3 (Fig. 2.6); radial cell 3.0 times as long as wide (Fig. 4.5). Male unknown ..................................Alloxysta basimacula (Cameron, 1886)

- Female: F2 shorter than F1, F2 longer, shorter than or subequal to F3; radial cell longer or shorter

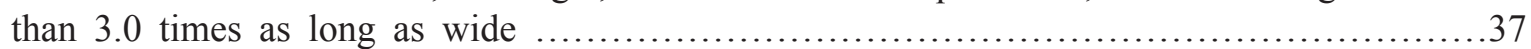

37. F2 longer than F3 (Fig. 2.7); radial cell 2.7 times as long as wide (Fig. 4.6) ........... Alloxysta brachycera Hellén, 1963

- F2 shorter than or subequal to F3; radial cell shorter or longer than 2.7 times as long as wide ...38

38. F2-F4 subequal in length (Fig. 2.15); radial cell 2.8 times as long as wide (Fig. 4.13)

- F2-F4 not subequal in length; radial cell shorter than 2.8 times as long as wide

39. F1 4.4 times as long as wide; F3 longer than F4 (Fig. 2.30); radial cell 2.9 times as long as wide, Rs and R1 reaching the costal margin (Fig. 5.2) ...........Alloxysta nigrita (Thomson, 1862)

- F1 1.4 times as long as wide, F3 subequal to F4 (Fig. 3.2); radial cell 2.8 times as long as wide; Rs and R1 not reaching the costal margin (Fig. 5.6) ...Alloxysta piceomaculata (Cameron, 1883)

40. Female: rhinaria and club shape begin on F4; F1 shorter than pedicel and slightly longer than F2; F2 subequal to F3; F3 shorter than F4 (Fig. 12.3); radial cell 2.0 times as long as wide (Fig. 12.1). Male unknown Alloxysta pascuali Ferrer-Suay sp. nov.

- Specimens without the same combination of features ...41

41. Female: rhinaria and club shape begin on F3; F1 2.9 times as long as wide; F2 shorter than F3; F3 subequal to F4 (Fig. 3.19). Male unknown ...Alloxysta xanthopa (Thomson, 1862)

- Rhinaria and club shape begin on F2, different proportion between flagellomeres ........42

42. Female: F1 5.2 times as long as wide; F2-F4 subequal in length (Fig. 2.32). Male: F2 slightly longer than F3; propodeum with two carinae well defined and separated on the anterior half with setae present, joining forming a plate in the posterior half; radial cell 2.6 times as long as wide (Fig. 5.4) .....Alloxysta pallidicornis (Curtis, 1838)

- Female: F1 4.1 times as long as wide; F2 longer than F3; F3 longer than F4 (Fig. 2.1). Male: F2 shorter than F3; propodeum with two carinae forming a thick plate, with setae on top and curved sides; radial cell 2.2 times as long as wide (Fig. 4.1) ...Alloxysta abdera Fergusson, 1986 


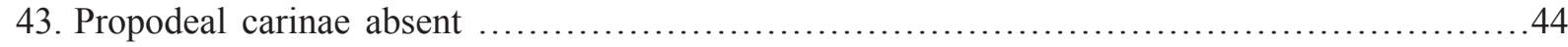

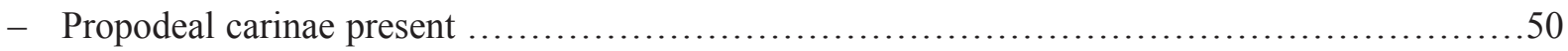

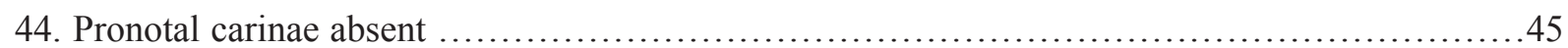

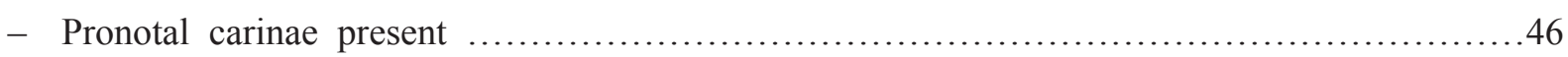

45. F1 longer than pedicel, F1 3.0 times as long as wide (Fig. 3.15); radial cell 2.3 times as long as wide; Rs reaching the costal margin (Fig. 5.19) Alloxysta soluta Hellén, 1963

- F1 subequal to pedicel and longer than F2; F2 subequal to F3 (Fig. 2.33); radial cell 2.8 times as long as wide, Rs not reaching the costal margin (Fig. 5.5) ...Alloxysta patens Hellén, 1963

46. Rhinaria and club shape begin on F4

- Rhinaria and club shape begin on F3

47. F2-F4 subequal in length

Alloxysta fuscipes (Thomson, 1862)

- F2-F4 not subequal in length

48. Female: F1 subequal to F2; F2 longer than F3; F3 subequal to F4 (Fig. 4.21). Male: F2 and F3 curved; F1 subequal to F2; F3 longer than F4; radial cell very big, 3.0 times as long as wide in both male and female (Fig. 8.6) ........Alloxysta macrophadna (Hartig, 1841)

- Female: F1 longer than F2; F2 shorter or subequal to F3; F3 shorter than F4 (Fig. 2.31). Male: without any flagellomere curved; F1 longer than F2; F3 shorter than F4; radial cell 2.7 times as long as wide in both male and female (Fig. 5.3) ......Alloxysta obscurata (Hartig, 1840)

49. F1 6.3 times as long as wide, F2 4.6 times as long as wide (Fig. 3.11); radial cell 2.6 times as long as wide (Fig. 5.15). Male unknown .......................Alloxysta salicicola Belizin, 1973

- F1 3.5 times as long as wide; F2 2.1 times as long as wide (Fig. 3.13); radial cell 2.7 times as long as wide (Fig. 5.17). Male: rhinaria and club shape begin on F4; F1 longer than F2; F2 longer than F3; F3 shorter than F4

Alloxysta semiaperta Fergusson, 1986

50. Propodeal carinae not protruding; F1 subequal to pedicel in both sexes; rhinaria and club shape begin on F4 (Fig. 2.13); radial cell 2.1 times as long as wide (Fig. 4.11) ...Alloxysta citripes (Thomson, 1862)

- Propodeal carinae well defined and protruding; without the same combination of features ...51

51. Propodeum with two carinae well defined, reaching the posterior part of the propodeum independently, thick and with the sides curved; rhinaria and club shape begin on F3 in female (Fig. 3.4); F1-F3 slightly curved in male; radial cell small with Rs vein straight (Fig. 5.8) ....

Alloxysta pleuralis (Cameron, 1879)

- Propodeum with two carinae forming a plate or only joining at the base; without the same

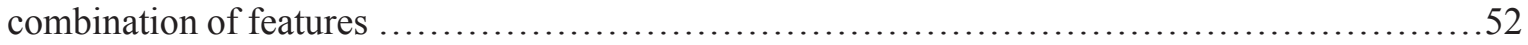

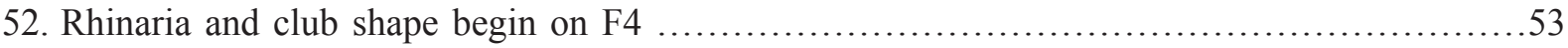

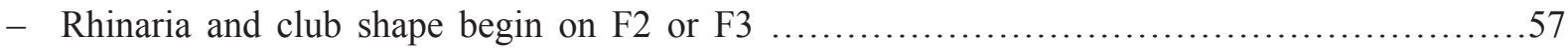

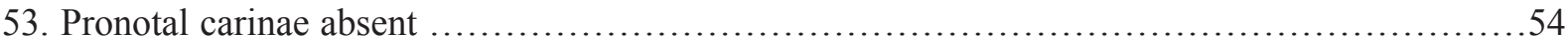

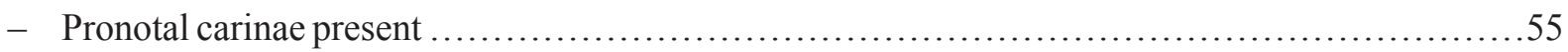

54. Female: pedicel-F3 subequal in length (Fig. 3.10); propodeum with two carinae forming a plate with straight sides Alloxysta rufiventris (Hartig, 1840)

- Female: pedicel-F3 not subequal in length (Fig. 3.14); propodeum with two carinae forming a plate with only slightly curved sides ......Alloxysta slovenica Ferrer-Suay \& Pujade-Villar, 2013 
55. F1 subequal to pedicel

Alloxysta postica (Hartig, 1841)

- F1 longer than pedicel

56. Female: F1 4.6 times as long as wide; F2 3.3 times as long as wide; F3 3.1 times as long as wide; F2 subequal to F3 (Fig. 2.11); radial cell 2.3 times as long as wide (sometimes the club shape begins on F3) (Fig. 4.9) ................................Alloxysta castanea (Hartig, 1841)

- F1 5.7 times as long as wide; F2 5.0 times as long as wide; F3 5.7 times as long as wide; F2 shorter than F3 (Fig. 2.5); radial cell 3.0 times as long as wide (Fig. 4.4). Male unknown...... Alloxysta aurata Belizin, 1968

57. Female: pedicel-F3 subequal in length (Fig. 2.27). Male: F1 longer than pedicel and F2; F2 subequal in F3; radial cell 2.0 times as long as wide (Fig. 4.23) ...Alloxysta melanogaster (Hartig, 1840)

- Female: F1 longer than pedicel and F2; radial cell 2.6 times as long as wide. Male unknown Alloxysta longipennis (Hartig, 1841)

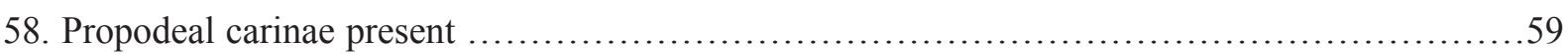

- Propodeal carinae absent ........................................................... 70

59. Propodeal carinae independent, slightly fused posteriorly

Alloxysta barbotini Ferrer-Suay \& Pujade-Villar, 2016

- Propodeal carinae fused forming a plate .....................................................60

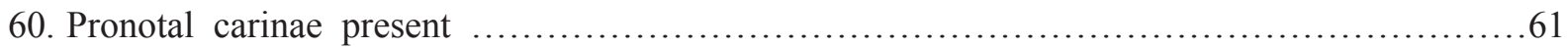

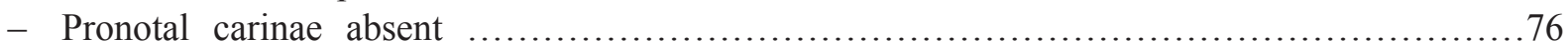

61. Female unknown. Male: radial cell 1.8 times as long as wide; club shape begins on F2 and rhinaria on F3; F1 longer than pedicel and F2; F2 longer than F3 (Fig. 2.10); all flagellomeres straight; propodeal carinae with curved sides .......................Alloxysta brevitarsis (Thomson, 1862)

- Radial cell longer than 1.8 times as long as wide; without the same combination of features ...62

62. Rhinaria and club shape begin on different flagellomeres; club shape begins on F2 and rhinaria on F1: F2 subequal to F3 (Fig. 3.18). Male unknown ......Alloxysta xanthocera (Thomson, 1862)

- Rhinaria and club shape begin on the same flagellomeres ................................63

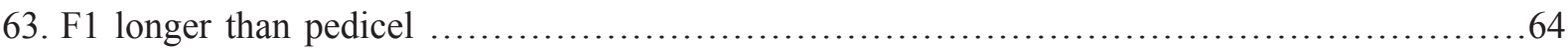

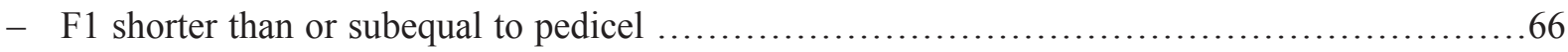

64. Female: rhinaria and club shape begin on F4; radial cell 2.7 times as long as wide (Fig. 5.1). Male unknown. Propodeal carinae forming a complete plate with few setae anteriorly and with divergent peaks posteriorly ................................Alloxysta nigricans Hellén, 1963

- Female: rhinaria and club shape begin on F3; with different combination of features ...65

65. Female: F2 shorter than F3 (Fig. 3.7). Male: rhinaria begin on F1; pedicel-F3 subequal and slightly curved; radial cell 2.7 times as long as wide in female (Fig. 5.11) and 2.4 times in male Alloxysta pusilla (Kieffer, 1902)

- Female: F2 subequal to F3 (Fig. 3.3). Male: rhinaria begin on F2; pedicel-F3 not subequal, without any flagellomere curved; radial cell 2.4 times as long as wide (Fig. 5.7) ........ Alloxysta pilipennis (Hartig, 1840)

66. F1 shorter than pedicel Alloxysta heptatoma Hellén, 1963

- F1 subequal to pedicel 
67. Rhinaria and club shape begin on F5; propodeal carinae narrow and well defined in the anterior half, wide and forming a plate in posterior half with sharp margins

Alloxysta sawoniewiczi Kierych, 1988

- Rhinaria and club shape begin on F3 or F4; propodeal carinae form a complete plate with curved

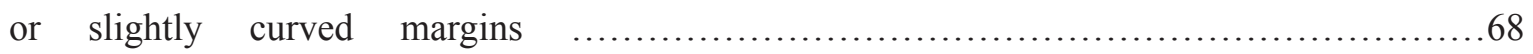

68. Rhinaria and club shape begin on F4; F1 longer than F2; F2 subequal to F3; with two small carinae on the pronotum, sometimes difficult to see (Fig. 3.9); propodeum with two carinae separed by setae on the anterior third and forming a plate in the posterior two thirds; radial cell 2.0 times as long as wide (Fig. 5.13) ......Alloxysta ramulifera (Thomson, 1862)

- Rhinaria and club shape begin on F3; without the same combination of features .....69

69. Female: F3 shorter than F4 (Fig. 2.4). Male: F1 longer than pedicel and subequal to F2; F2 slightly curved and shorter than F3; radial cell 2.3 times as long as wide (Fig. 4.3)

Alloxysta arcuata (Kieffer, 1902)

- Female: F3-F4 subequal in length (Fig. 2.16); radial cell 2.6 times as long as wide (Fig. 4.14). Male unknown .......................................Alloxysta crassicornis (Thomson, 1862)

70. Head yellow; F1 longer than F2, F2-F4 subequal (Fig. 3.17); radial cell 3.0 times as long as wide (Fig. 5.21); propodeum without setae where they are usually present in other species (Fig. 9.2) ......

Alloxysta victrix (Westwood, 1833)

- Head brown; without the same combination of features .................................. 71

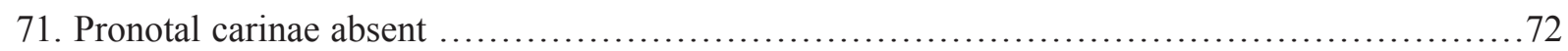

- Pronotal carinae present ........................................................... 74

72. F1-F3 not subequal in length ...........Alloxysta kovilovica Ferrer-Suay \& Pujade-Villar, 2013

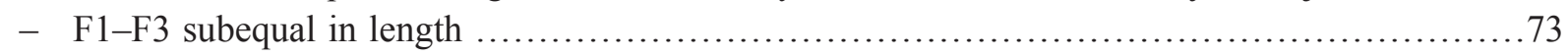

73. Female: rhinaria and club shape begin on F4; F1 longer than pedicel (Fig. 2.2); radial cell 2.4 times as long as wide (Fig. 4.2). Male with antenna unknown ...Alloxysta aperta (Hartig, 1841)

- Female unknown. Male: rhinaria and club shape begin on F3; F1 subequal to pedicel (Fig. 3.8); radial cell 2.5 times as long as wide (Fig. 5.12) ........Alloxysta quedenfeldti (Kieffer, 1909)

74. Female: rhinaria and club shape begin on F3; F1 longer than pedicel and subequal to F2; F2 shorter than or subequal to F3 (Fig. 2.12). Male: F1-F3 not curved

Alloxysta circumscripta (Hartig, 1841)

- Rhinaria and club shape begin on other flagellomere; without the same combination of features ...75

75. Female: rhinaria and club shape begin on F3 or F4; F1 longer than pedicel and F2; F2 subequal to F3 (Fig. 2.14). Male: F1-F3 curved; radial cell 2.7 times as long as wide (Fig. 4.12)

Alloxysta consobrina (Zetterstedt, 1838)

Alloxysta tscheki (Giraud, 1860)

- Female: rhinaria and club shape begin on F2; F1 longer than pedicel and F2; F2 shorter than F3 (Fig. 2.23). Male: F1 curved; radial cell 2.0 times as long as wide (Fig. 4.19)

76. F1 longer than pedicel

A. fracticornis (Thomson, 1862)

- F1 shorter than or subequal to pedicel 
77. Male and female: F1 subequal to pedicel and longer than F2; F2 subequal to F3 (Fig. 2.28) ... Alloxysta mullensis (Cameron, 1883)

- F1 shorter than pedicel, F1-F3 subequal in length, sometimes F1 slightly longer ...............78

78. Antenna shorter than body length; fore wing with normal marginal setae (Fig. 10.2)

Alloxysta brevis (Thomson, 1862)

- Antenna subequal or longer than body length; fore wing with long marginal setae (Fig. 10.3) ... Alloxysta darci (Girault, 1933)

\section{Alloxysta abdera Fergusson, 1986}

Figs 2.1, 4.1

Alloxysta abdera Fergusson, 1986: 10. Type: deposited in BMNH (examined).

\section{Diagnosis}

Alloxysta abdera is mainly characterized by a completely open radial cell that is 2.2 times as long as wide in both males and females (Fig. 4.1); the presence of pronotal and propodeal carinae; female antenna with rhinaria beginning on F4, F1 longer than the pedicel and subequal to F2, F2 longer than F3, and F3 slightly longer than F4 (Fig. 2.1); male antenna with rhinaria beginning on F2, F2 curved, F1 longer than pedicel and F2, F2 shorter than F3, and F3 subequal to F4. It is similar to Alloxysta pallidicornis (Curtis, 1838), but can be differentiated by the flagellomere proportions in females (F2 is longer than F3 and F3 is slightly longer than F4 in A. abdera (Fig. 2.1), but F2-F4 are subequal in length in A. pallidicornis (Fig. 2.32)), the shape of the propodeal carinae (they form a plate with setae on top and sides slightly curved in A. abdera, while the two carinae are well-defined, separated anteriorly, and joined at the base in A. pallidicornis), and the size of the radial cell (2.2 times as long as wide in A. abdera (Fig. 4.1) but 2.6 times in A. pallidicornis (Fig. 5.4)).

\section{Material examined}

\section{Holotype}

UNITED KINGDOM: England: đ [Lancs. woodland, Belmont, 25 Sep. 1981, Holotype (round label with red margins)], [§]], [Holotype of Alloxysta abdera det. N.D.M. Fergusson, 1984, B.M. Type Hym. 7. 175] (BMNH B.M. Type Hym. 7. 175).

\section{Paratype}

UNITED KINGDOM: England: 1 q [Lancs., woodland, Belmont, 25 Sep. 1981, Alloxystinae (handwritten)], [Paratype (round label with yellow margins), A. abdera det. N.D.M. Fergusson] (BMNH).

\section{Distribution}

Europe.

Certain records: England (Fergusson 1986: 19), France (Ferrer-Suay et al. 2015a).

Alloxysta aperta (Hartig, 1841)

Figs 2.2, 4.2

Xystus apertus Hartig 1841: 353. Type: deposited in ZSM (examined).

Allotria aperta - Thomson 1862: 410.

Allotria apertus - Taschenberg 1866: 129. 

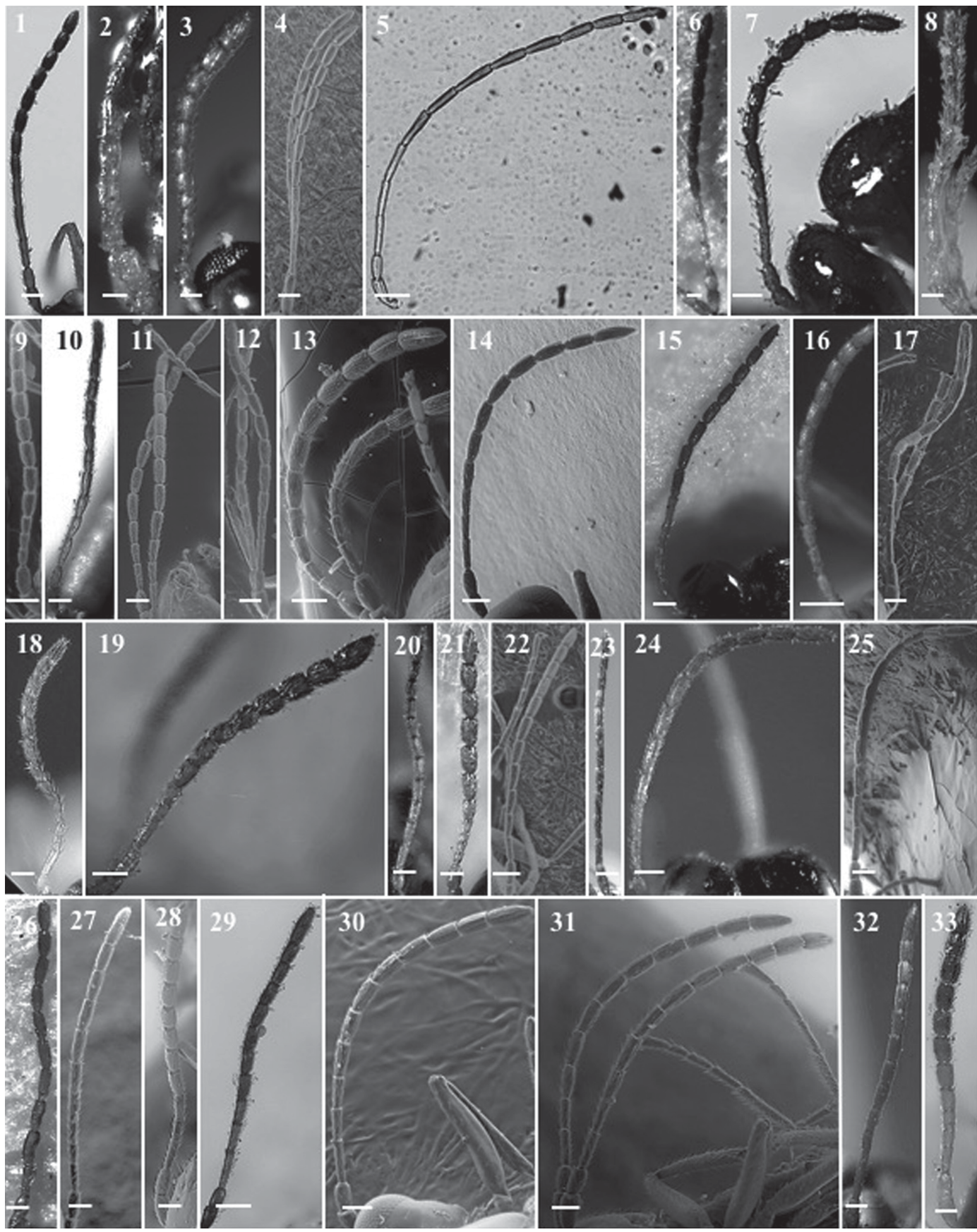

Fig. 2. Types of antenna of Alloxysta Förster, 1869. 1. A. abdera Fergusson, 1986. 2. A. aperta (Hartig, 1841). 3. A. apteroidea Hellén, 1963. 4. A. arcuata (Kieffer, 1902). 5. A. aurata Belizin, 1968. 6. A. basimacula (Cameron, 1886). 7. A. brachycera Hellén, 1963. 8. A. brachyptera (Hartig, 1840). 9. A. brevis (Thomson, 1862). 10. A. brevitarsis (Thomson, 1862). 11. A. castanea (Hartig, 1841). 12. A. circumscripta (Hartig, 1841). 13. A. citripes (Thomson, 1862). 14. A. consobrina (Zetterstedt, 1838). 15. A. crassa (Cameron, 1889). 16. A. crassicornis (Thomson, 1862). 17. A. fracticornis (Thomson, 1862). 18. A. fuscipes (Thomson, 1862). 19. A. glebaria Hellén, 1963. 20. A. halterata (Thomson, 1862). 21. A. heptatoma Hellén, 1963. 22. A. kovilovica Ferrer-Suay \& Pujade-Villar, 2013. 23. A. leunisii (Hartig, 1841). 24. A. longipennis (Hartig, 1841). 25. A. macrophadna (Hartig, 1841). 26. A. marshalliana (Kieffer, 1900). 27. A. melanogaster (Hartig, 1840). 28. A. mullensis (Cameron, 1883). 29. A. nigricans Hellén, 1963. 30. A. nigrita (Thomson, 1862). 31. A. obscurata (Hartig, 1840). 32. A. pallidicornis (Curtis, 1838). 33. A. patens Hellén, 1963. Scale bars: $50 \mu \mathrm{m}$. 


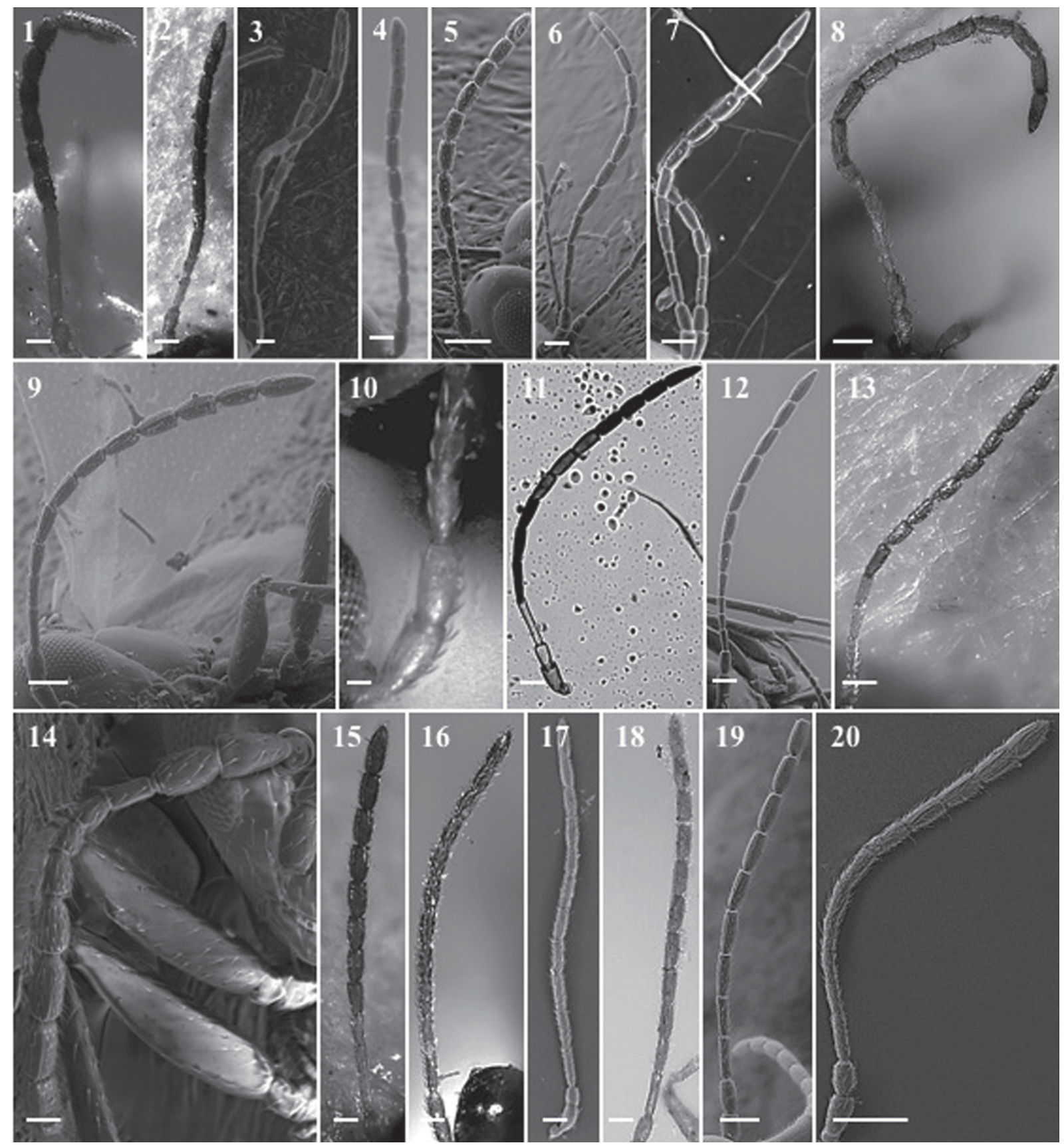

Fig. 3. Types of antenna of Alloxysta Förster, 1869. 1. A. pedestris (Curtis, 1838). 2. A. piceomaculata (Cameron, 1883). 3. A. pilipennis (Hartig, 1840). 4. A. pleuralis (Cameron, 1879). 5. A. postica (Hartig, 1841). 6. A. proxima Belizin, 1962. 7. A. pusilla (Kieffer, 1902). 8. A. quedenfeldti (Kieffer, 1909). 9. A. ramulifera (Thomson, 1862). 10. A. rufiventris (Hartig, 1840). 11. A. salicicola Belizin, 1973. 12. A. sawoniewiczi Kierych, 1988. 13. A. semiaperta Fergusson, 1986. 14. A. slovenica Ferrer-Suay \& Pujade-Villar, 2013. 15. A. soluta Hellén, 1963. 16. A. tscheki (Giraud, 1860). 17. A. victrix (Westwood, 1833). 18. A. xanthocera (Thomson, 1862). 19. A. xanthopa (Thomson, 1862). 20. A. trapezoidea (Hartig, 1841). Scale bars: $50 \mu \mathrm{m}$. 

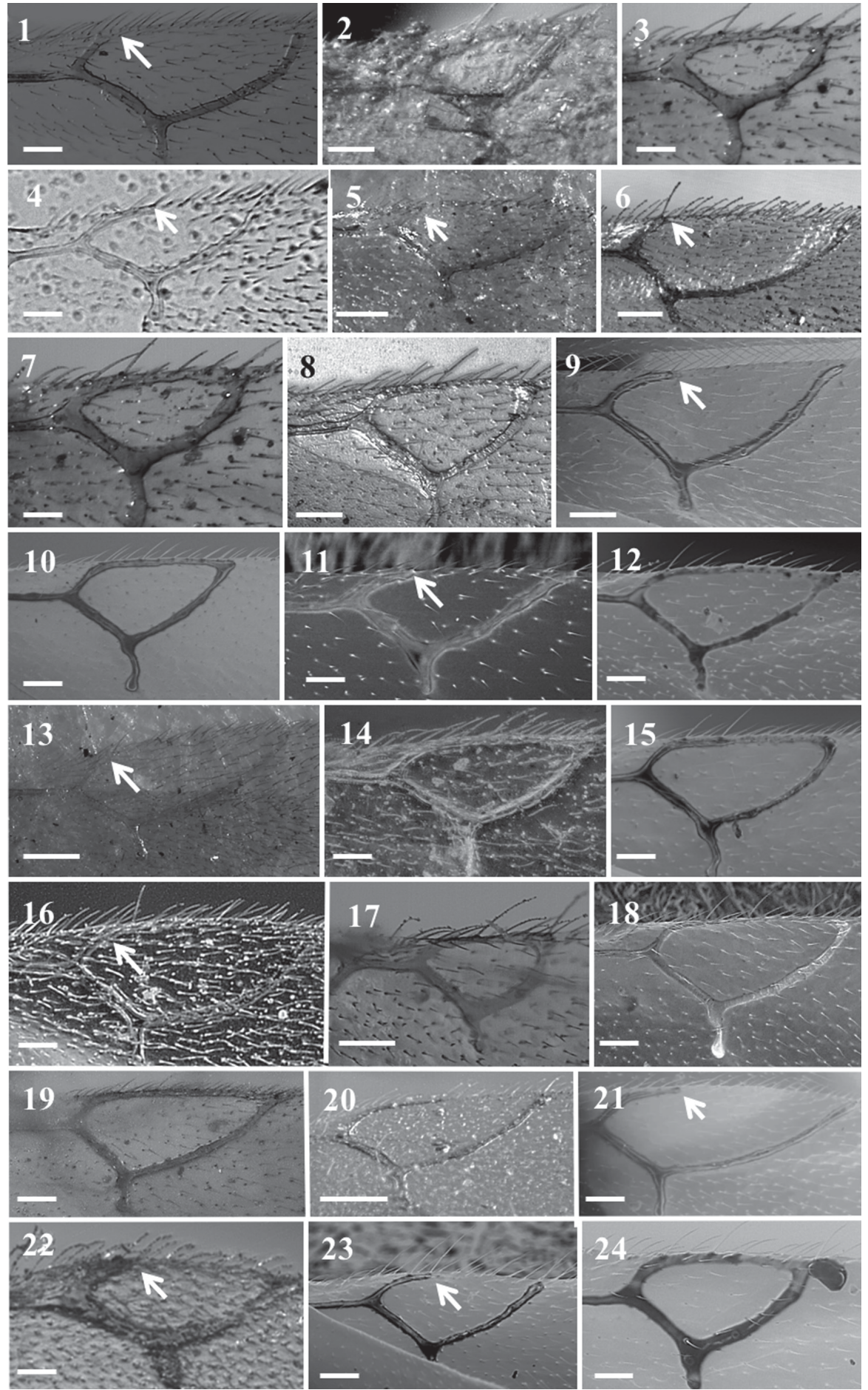

Fig. 4. Types of radial cell of Alloxysta Förster, 1869. 1. A. abdera Fergusson, 1986. 2. A aperta (Hartig, 1841). 3. A. arcuata (Kieffer, 1902). 4. A. aurata Belizin, 1968. 5. A. basimacula (Cameron, 1886). 6. A. brachycera Hellén, 1963. 7. A. brevis (Thomson, 1862). 8. A. brevitarsis (Thomson, 1862). 9. A. castanea (Hartig, 1841). 10. A. circumscripta (Hartig, 1841). 11. A. citripes (Thomson, 1862). 12. A. consobrina (Zetterstedt, 1838). 13. A. crassa (Cameron, 1889). 14. A. crassicornis (Thomson, 1862). 15. A. fracticornis (Thomson, 1862). 16. A. fuscipes (Thomson, 1862). 17. A. heptatoma Hellén, 1963. 18. A. kovilovica Ferrer-Suay \& Pujade-Villar, 2013. 19. A. leunisii (Hartig, 1841). 20. A. longipennis (Hartig, 1841). 21. A. macrophadna (Hartig, 1841). 22. A. marshalliana (Kieffer, 1900). 23. A. melanogaster (Hartig, 1840). 24. A. mullensis (Cameron, 1883). Scale bars: $50 \mu \mathrm{m}$. 

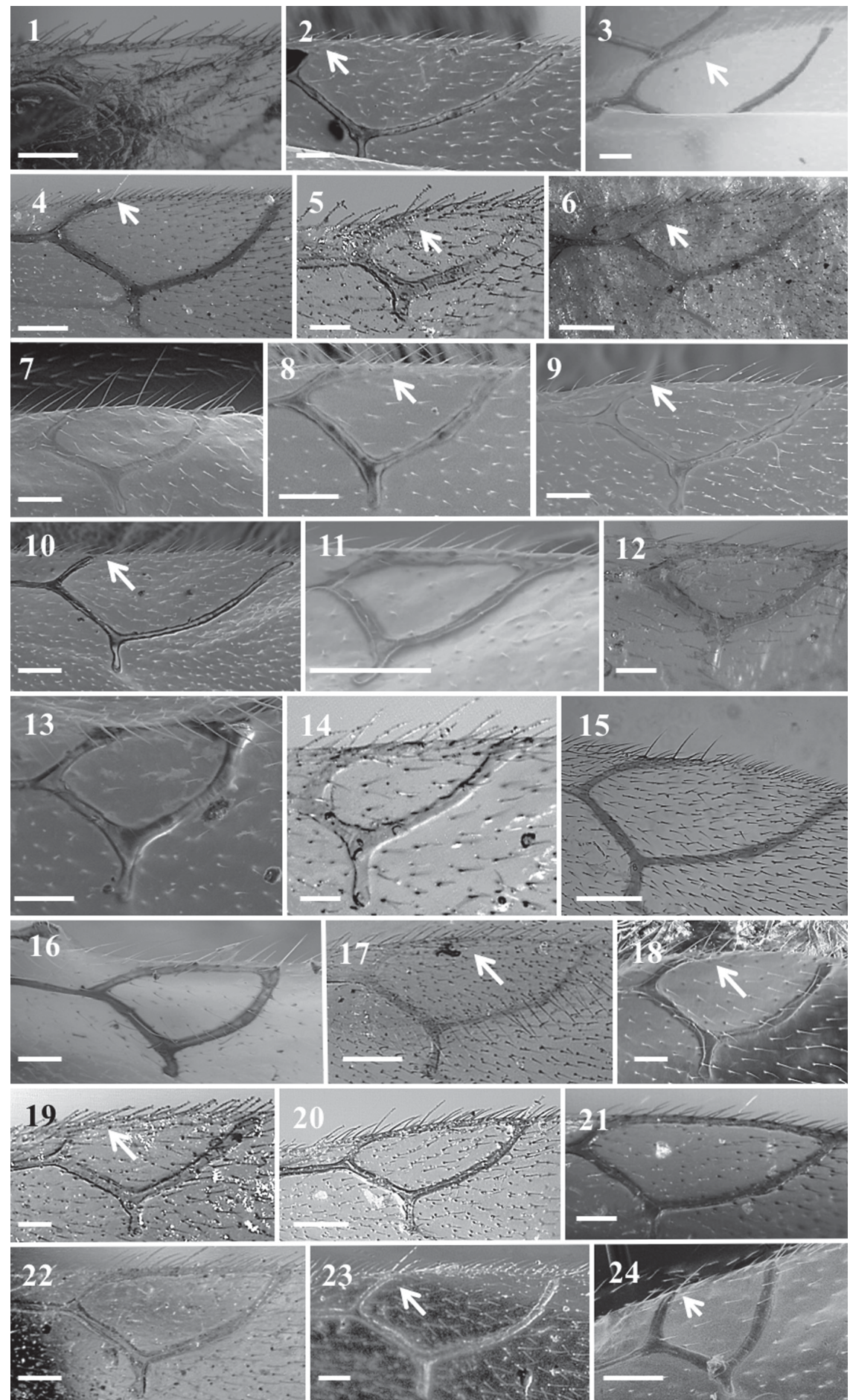

Fig. 5. Types of radial cell of Alloxysta Förster, 1869. 1. A. nigricans Hellén, 1963. 2. A. nigrita (Thomson, 1862). 3. A. obscurata (Hartig, 1840). 4. A. pallidicornis (Curtis, 1838). 5. A. patens Hellén, 1963. 6. A. piceomaculata (Cameron, 1883). 7. A. pilipennis (Hartig, 1840). 8. A. pleuralis (Cameron, 1879). 9. A. postica (Hartig, 1841). 10. A. proxima Belizin, 1962. 11. A. pusilla (Kieffer, 1902). 12. A. quedenfeldti (Kieffer, 1909). 13. A. ramulifera (Thomson, 1862). 14. A. rufiventris (Hartig, 1840). 15. A. salicicola Belizin, 1973. 16. A. sawoniewiczi Kierych, 1988. 17. A. semiaperta Fergusson, 1986. 18. A. slovenica Ferrer-Suay \& Pujade-Villar, 2013. 19. A. soluta Hellén, 1963. 20. A. tscheki (Giraud, 1860). 21. A. victrix (Westwood, 1833). 22. A. xanthocera (Thomson, 1862). 23. A. xanthopa (Thomson, 1862). 24. A. trapezoidea (Hartig, 1841). Scale bars: $50 \mu \mathrm{m}$. 

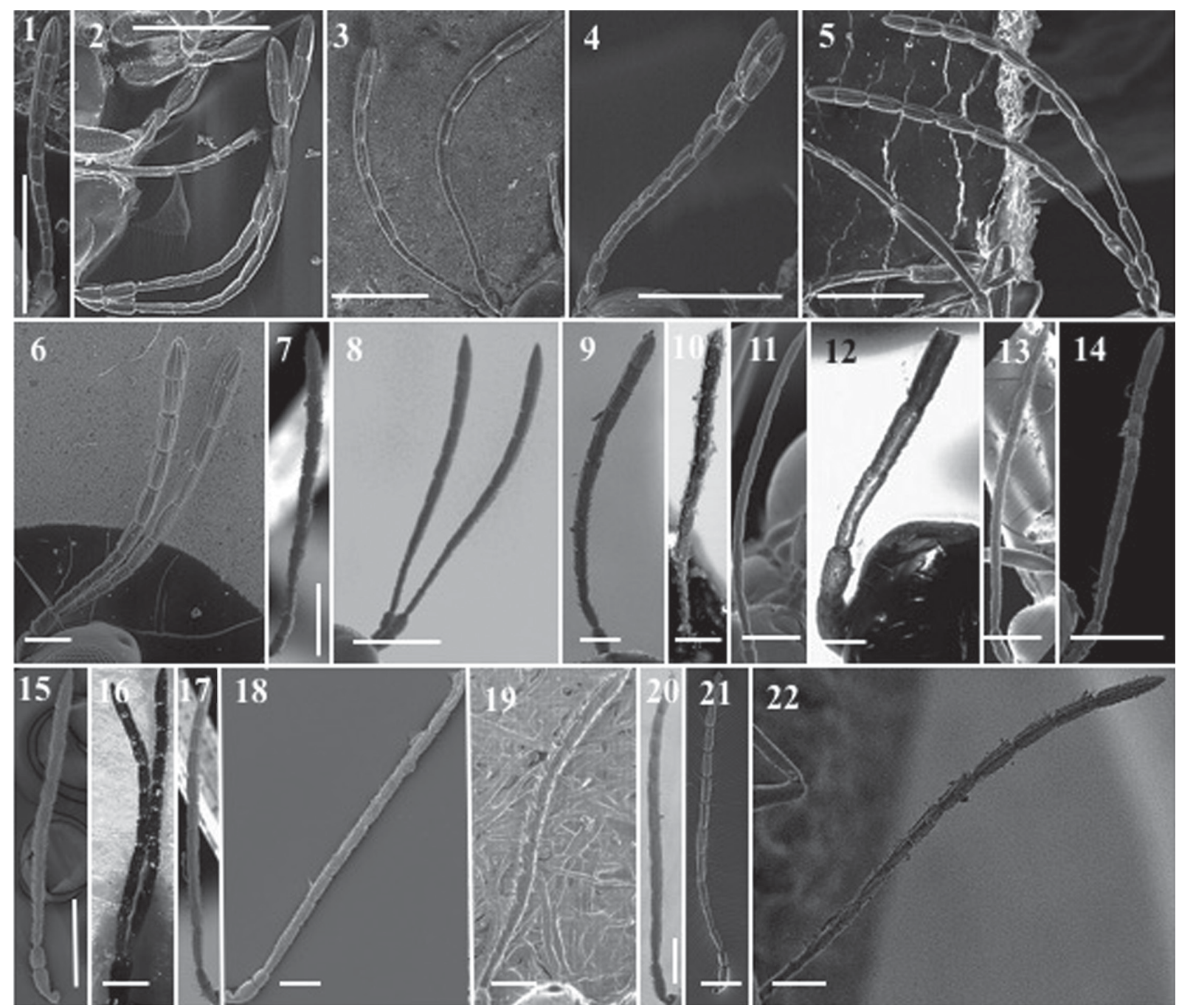

Fig. 6. Types of antenna of Dilyta Förster, 1869 and Phaenoglyphis Förster, 1869. 1. D. aleevae PujadeVillar \& Paretas-Martínez, 2011. 2. D. japonica Paretas-Martínez \& Ferrer-Suay, 2011. 3. D. longinqua Paretas-Martínez \& Pujade-Villar, 2011. 4. D. rathmanae. 5. D. sinica Ferrer-Suay \& Paretas-Martínez, 2011. 6. D. subclavata Förster, 1869. 7. P. abbreviata (Thomson, 1877). 8. P. evenhuisi PujadeVillar \& Paretas-Martínez, 2006. 9. P. fuscicornis (Thomson, 1877). 10. P. heterocera (Hartig, 1841). 11. P. insperatus Belizin, 1973. 12. P. insularis (Belizin, 1973). 13. P. longicornis (Hartig, 1840). 14. P. moldavica Ionescu, 1969. 15. P. nigripes (Thomson, 1877). 16. P. proximus Belizin, 1966. 17. P. pubicollis (Thomson, 1877). 18. P. ruficornis (Förster, 1869). 19. P. salicis (Cameron, 1883). 20. P. stricta (Thomson, 1877). 21. P. villosa (Hartig, 1841). 22. P. xanthochroa Förster, 1869. Scale bars: $50 \mu \mathrm{m}$. 

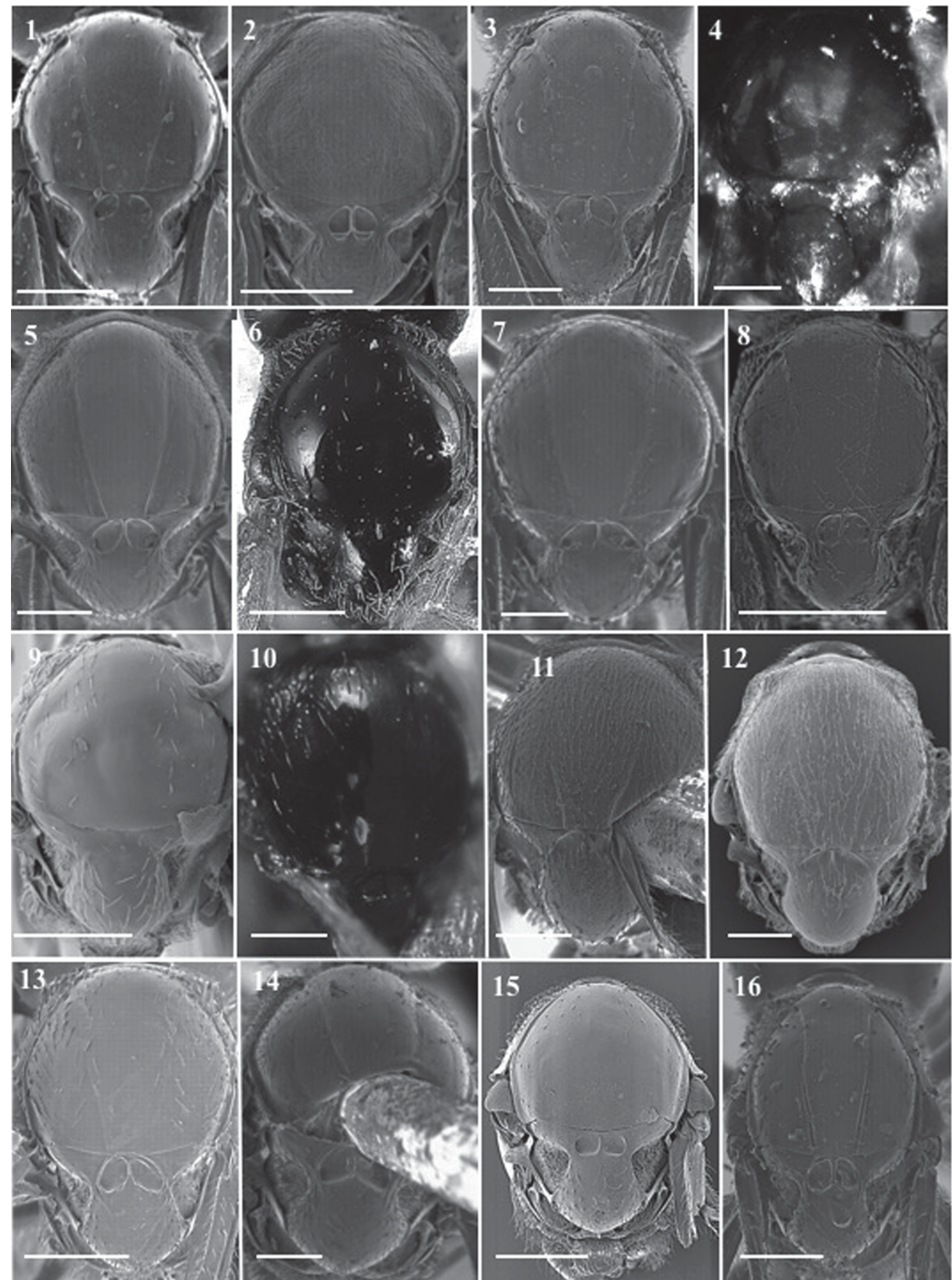

Fig. 7. Types of mesoscutum of Phaenoglyphis Förster, 1869. 1. P. abbreviata (Thomson, 1877). 2. P. evenhuisi Pujade-Villar \& Paretas-Martínez, 2006. 3. P. fuscicornis (Thomson, 1877). 4. P. heterocera (Hartig, 1841). 5. P. insperatus Belizin, 1973. 6. P. insularis (Belizin, 1973). 7. P. longicornis (Hartig, 1840). 8. P. moldavica Ionescu, 1969. 9. P. nigripes (Thomson, 1877). 10. P. proximus Belizin, 1966. 11. P. pubicollis (Thomson, 1877). 12. P. ruficornis (Förster, 1869). 13. P. salicis (Cameron, 1883). 14. P. stricta (Thomson, 1877). 15. P. villosa (Hartig, 1841). 16. P. xanthochroa Förster, 1869. Scale bars: $50 \mu \mathrm{m}$. 

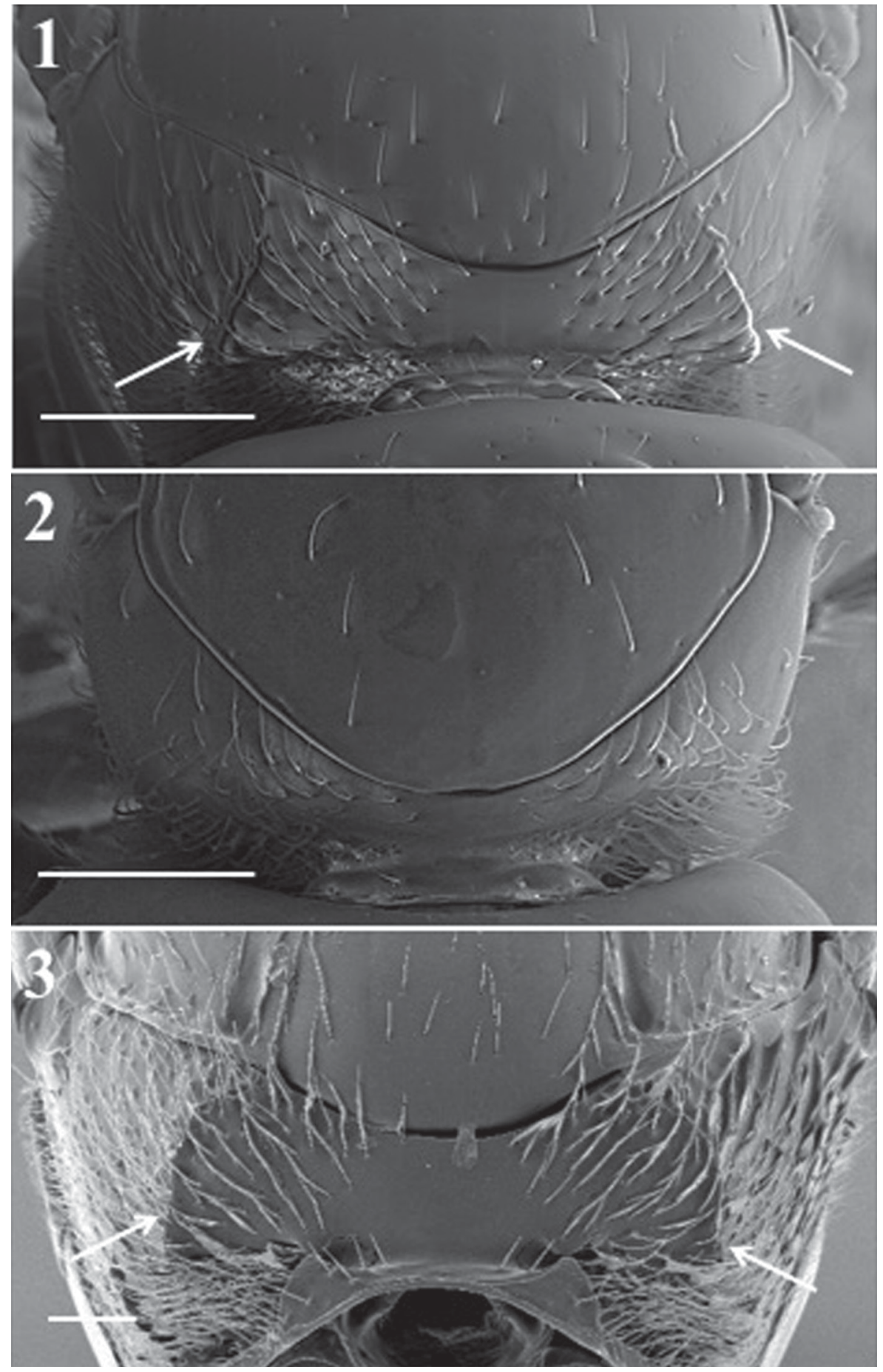

Fig. 8. Types of pronotum. 1. Alloxysta arcuata (Kieffer, 1902). 2. A. brevis (Thomson, 1862). 3. Phaenoglyphis americana Baker, 1896. Scale bars: $50 \mu \mathrm{m}$. 

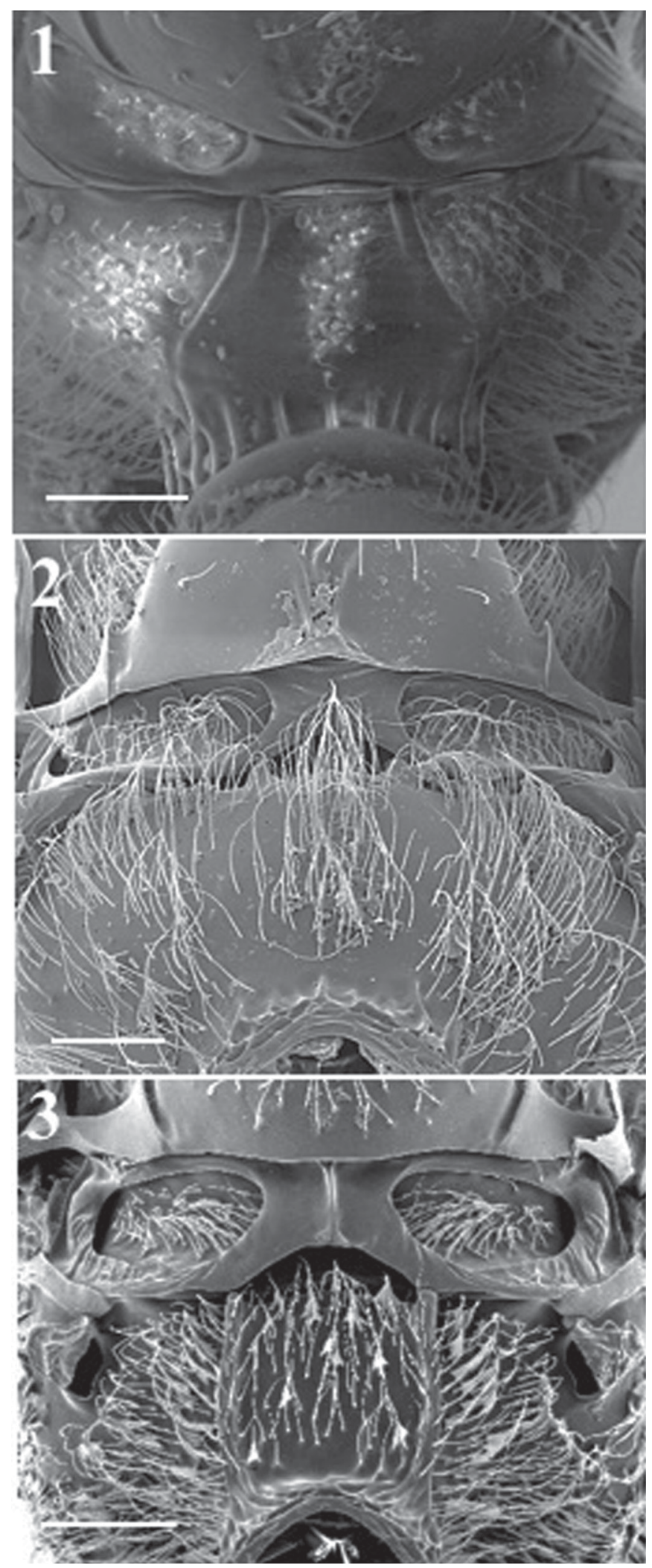

Fig. 9. Types of propodeum. 1. Alloxysta arcuata (Kieffer, 1902). 2. A. victrix (Westwood, 1833). 3. Phaenoglyphis americana Baker, 1896. Scale bars: $50 \mu \mathrm{m}$. 

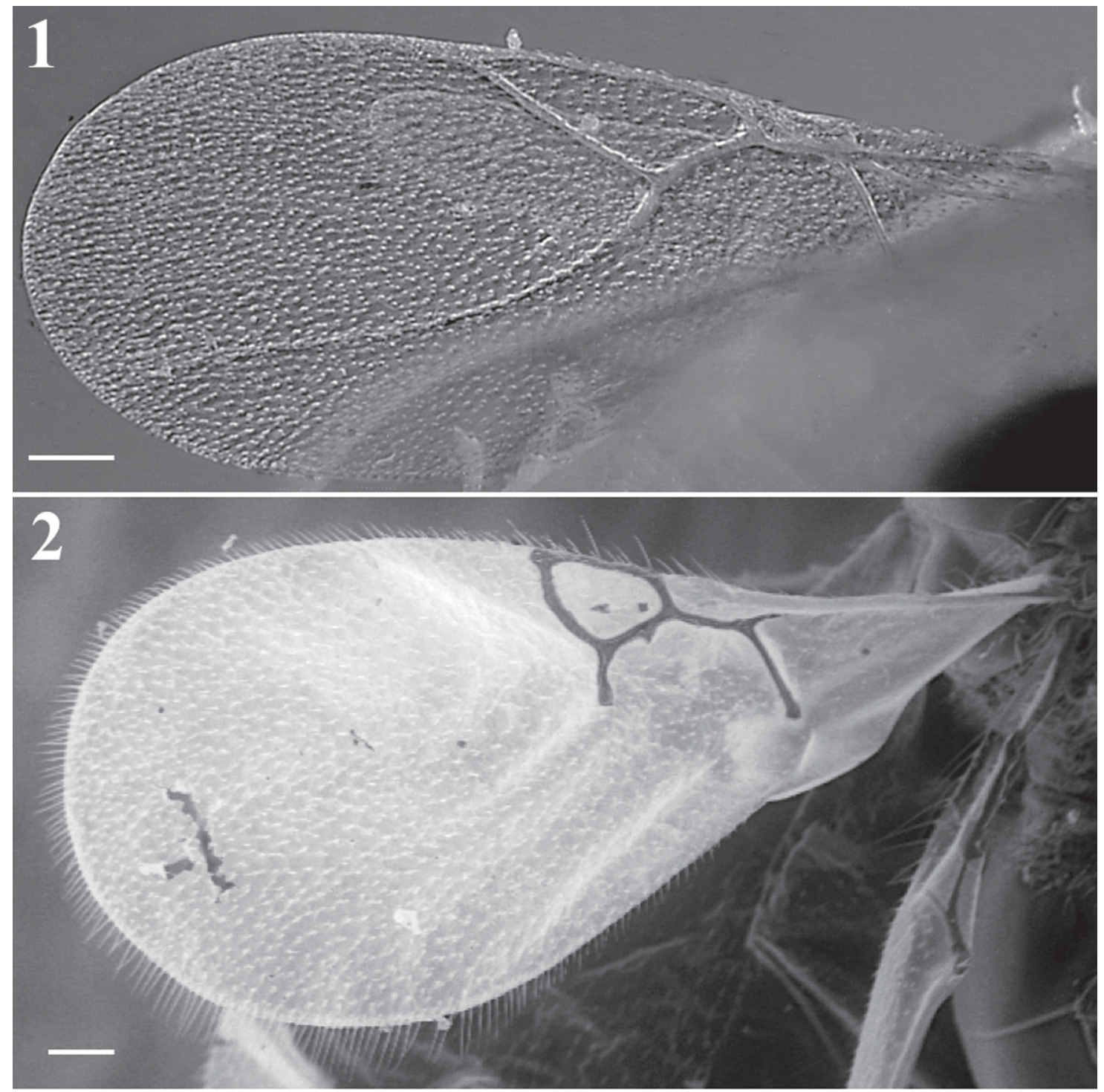

3

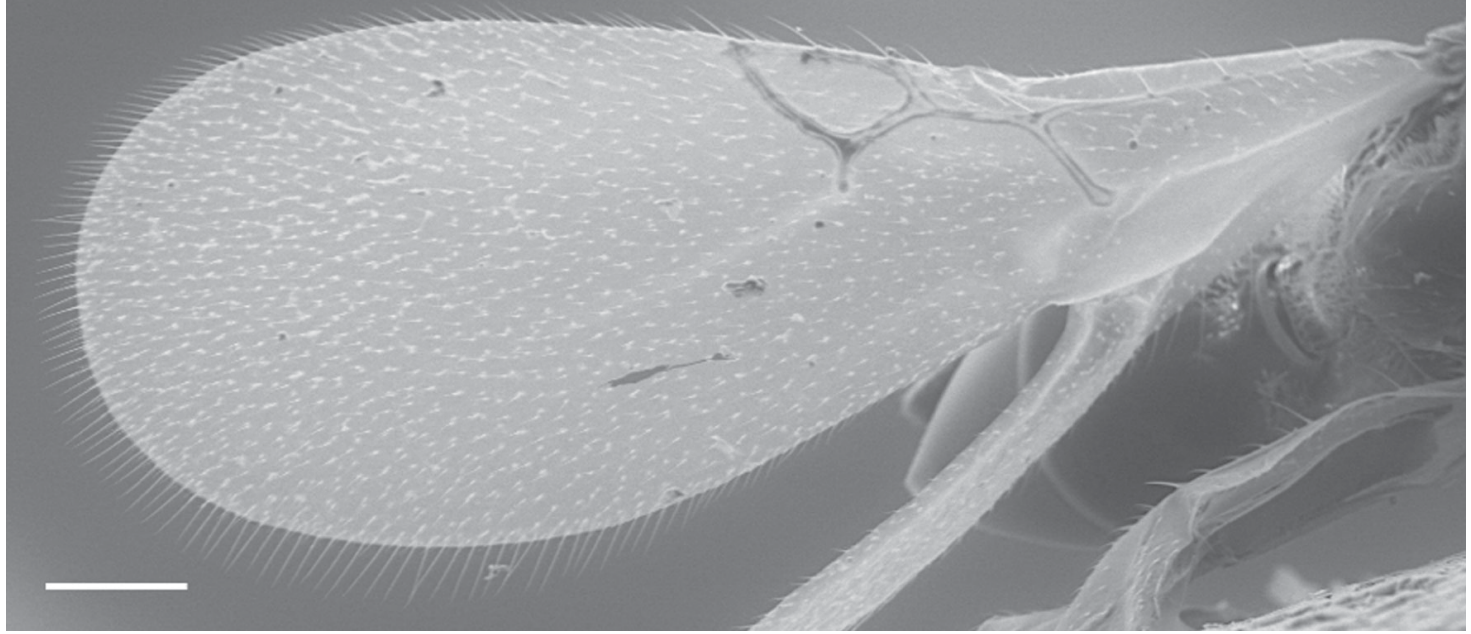

Fig. 10. Types of fore wing. 1. Phaenoglyphis evenhuisi Pujade-Villar \& Paretas-Martínez, 2006. 2. Alloxysta brevis (Thomson, 1862). 3. A. darci (Girault, 1933). Scale bars: $50 \mu \mathrm{m}$. 
Dilyta aperta - Kieffer 1900: 114.

Alloxysta (Alloxysta) aperta - Dalla Torre \& Kieffer 1902: 38.

Alloxysta aperta - Hellén 1931: 4.

\section{Diagnosis}

Alloxysta aperta is mainly characterized by a closed radial cell that is 2.4 times as long as wide (Fig. 4.2); the absence of pronotal and propodeal carinae; and female antenna with rhinaria beginning on F4, F1 slightly longer than pedicel, and F1-F3 subequal in length (Fig. 2.2). It is similar to A. quedenfeldti, but they differ in the proportion of pedicel-F1 (F1 is longer than pedicel in A. aperta (Fig. 2.2), but subequal to pedicel in A. quedenfeldti (Fig. 3.8)) and where rhinaria begin (on F4 in A. aperta (Fig. 2.2), but on $\mathrm{F} 3$ in A. quedenfeldti (Fig. 3.8)).

\section{Material examined}

\section{Lectotype}

GERMANY: + [lectotype H.H. Evenhuis (orange label), Xystus apertus Hartig det. H.H. Evenhuis 1980], [X. apertus Hart. $\widehat{\jmath}$ det. E. Kierych 1985], [Alloxysta aperta (Hartig, 1841) ㅇ M. Ferrer-Suay det. 2011] (ZSM).

\section{Distribution}

Europe.

Certain records: France (Evenhuis 1982: 21), Germany (Hartig 1841: 353).

Uncertain records: England (Andrews 1978: 78), Sweden (Thomson 1862: 410).

\section{Alloxysta apteroidea Hellén, 1963}

Fig. 2.3

Alloxysta apteroidea Hellén, 1963: 23. Type: deposited in MZH (examined).

\section{Diagnosis}

Alloxysta apteroidea is mainly characterized as a brachypterous species and by the absence of a visible radial cell; the fore wing being practically absent; the absence of pronotal and propodeal carinae; and antenna with F1 shorter than pedicel and F1 longer than F2 and subequal to F3 (Fig. 2.3). It is similar to $A$. pedestris, but can be differentiated by the length of the fore wings (which are very short and practically absent in A. apteroidea, while they reach the beginning of the metasoma in A. pedestris) and the proportion of F1-pedicel (F1 is shorter than pedicel in A. apteroidea (Fig. 2.3), but F1 is longer than pedicel in A. pedestris (Fig. 3.1)).

\section{Material examined}

\section{Holotype}

FINLAND: + [Runsala, Hellén, 157, apteroidea (handwritten)], [Typus Alloxysta apteroidea Hellén (handwritten) (red label)], [Mus. Zool. Helsinki, Loan No. HY 2012 - 1834 (yellow label)], [Alloxysta apteroidea Hellén, 1963 q M. Ferrer-Suay det. 2012] (MZH).

\section{Distribution}

Europe.

Certain records: Finland (Hellén 1963: 23). 
Alloxysta arcuata (Kieffer, 1902)

Figs $2.4,4.3,8.1,9.1$

Alloxysta minuta (Hartig, 1840) det. Cameron (misidentification). Type: deposited in BMNH (examined). Allotria (Allotria) arcuata Kieffer, 1902: 12.

Charips (Charips) arcuatus - Dalla Torre \& Kieffer 1910: 277.

Alloxysta arcuata - Evenhuis \& Barbotin 1977: 189.

\section{Diagnosis}

Alloxysta arcuata is mainly characterized by a small closed radial cell that is 2.3 times as long as wide (Fig. 4.3); the presence of pronotal carinae (Fig. 8.1); propodeal carinae that form a plate (Fig. 9.1); female antenna with rhinaria beginning on F3, F1 subequal to pedicel and longer than F2, and F2 subequal to F3 (Fig. 2.4); male antenna with rhinaria beginning on F2, F2 slightly curved, F1 longer than pedicel, F1 subequal to F2, and F2 shorter than F3. It is similar to A. ramulifera, but can be differentiated by the origin of the rhinaria (on F3 in A. arcuata (Fig. 2.4), but on F4 in A. ramulifera (Fig. 3.9)), the shape of the pronotal carinae (well-defined and visible in A. arcuata but small and sometimes difficult to see under the pubescence in $A$. ramulifera), the size of the radial cell (2.3 times as long as wide in A. arcuata (Fig. 4.3), but 2.0 in A. ramulifera (Fig. 5.13)), and the shape of the propodeal carinae (with curved sides in $A$. arcuata but with straight sides in $A$. ramulifera).

\section{Material examined}

\section{Lectotype}

UNITED KINGDOM: + [Lectotype (round label with blue in the margin)], [Cameron. 96-76., Clober Wood, Clober (handwritten)], [Allotria arcuata Kieffer nec Xystus minutus Hartig (handwritten, orange label)], [LECTOTYPE o of Allotria arcuata Kieffer. det. J. Quinlan, 1977 (white label), B. M. TYPE HYM (white label)], [Lectotype Allotria arcuata Kieffer, 1902 q design. Ferrer-Suay and J.P-V 2011 (red label)], [Alloxysta arcuata (Kieffer) , , Ferrer-Suay and J.P-V det. 2011 (white label)] (BMNH B.M. Type Hym.).

Additional specimens $(16 \hat{\partial} \hat{\partial}, 47 \stackrel{+}{\phi}$ 早)

CZECH REPUBLIC: 1 q [central Bohemia, 30 Jul. 1991, J. Macek] (CNCI C-278).

EGYPT: 1 [ [Helwan, 13.3.35, Egitto W. Wittmer, Received in exchange from G. Soika] (BMNH B.M. 1948-144).

FRANCE: 1 đ [Pyrénées Orientales nr. Arles-sur-Tech, D. and J. Clark, 22 May 1961, ð] (BMNH B.M.

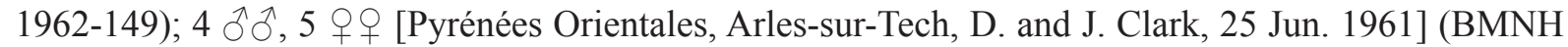
B.M. 1962-149); 1 q [Hérault, St. Vincent de Barbeyrargues, 4342'18" N, 353' E, 5-12 Jun. 1993, P.G. Mason, garrigue sauvage, YPT] (CNCI C-303) (handwritten).

GERMANY: 1 q [Munich, 23 Jul. 1984, R. Wharton: 1 đ; C-229, Mainz, 4-17 Sep. 1965, A.W. Stefan] (CNCI C-239); 1 o [Ingelheim am Rhein, ??.??.1968, MT in orchard, A.W. Steffan] (CNCI C-238);

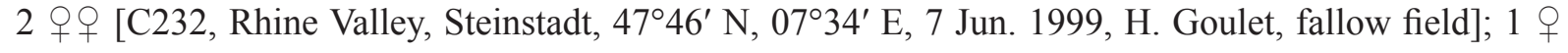
[Schwarzwald, Todtmoos environs, 6 Aug. 1984, L. Masner, screen sweeping] (CNCI C-235).

IRAN: 2 ふึ, 3 우 [Tehran Prov., Shahdasht, 25 Jun. 1978, J.T. Huber] (CNCI C-317).

ITALY: 2 우 [Abruzzo (AQ), L'Aquila, Aterno River, sweeping riparian veg. and Salix, 20 Jun. 1992, J.D. Pinto] (CNCI C-253). 
JAPAN: 1 [Ibaraki, Tsukuba, NIAES, 14-21 Jul. 1989, M.J. Sharkey, PT] (CNCI C-191); 1 [Ibariki, Tsukuba, Matsushiro, 26 Jul.-4 Aug. 1989, pan traps, M.J. Sharkey] (CNCI C-153); 1 \& [Hokkaido, Sapporo, Jozankei, 350 m, 20--31 Jul. 1989, K. Maeto and M. Sharkey] (CNCI C-151); 1 \& [Hokkaido,

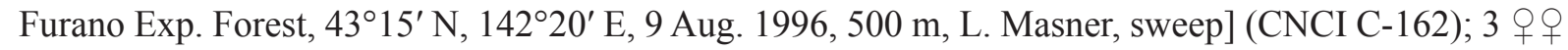
[Aichi, Nagoya, Ogawa, 11 Jun. 1984, R. Baczyushi] (CNCI C-179); 1 q [Hokkaido, Furano, Exp. Forest, $43^{\circ} 15^{\prime} \mathrm{N}, 142^{\circ} 20^{\prime} \mathrm{E}, 9$ Aug. 1996, $500 \mathrm{~m}$, L. Masner, sweep] (CNCI C-163); 1 ○ [Aichi, Shitara, Uradani, 900 m, 9-15 May 1994, K. Yamagishi, EMT, beech forest] (CNCI C-218); 2 우 [Hokkaido, Sapporo, Jozankei, 350 m, 10-21 Aug. 1989, K. Maeto and M. Sharkey, MT] (CNCI C-206).

MOROCCO: 1 [Marrakech, Ouirgane, 1000 m, 31 $08^{\circ}$ N, $8^{\circ} 05^{\prime}$ W, 19-25 Mar. 1997, C. Kassebeer, MT] (CNCI C-281).

POLAND: 1 q [Borowki, N.W. Poland, 16-28 Sep. 1934, G.J. Kerrich, A. brevis det. N.D.M. Fergusson] (BMNH) (handwritten).

RUSSIA: 1 ㅇ [Primoskiy Kray Ussyriysk District, Gomotayozhnoye, 11-15 Aug. 2003, Malaise trap, M.V. Michailovskaya] (USNM); 1 đ̊, 1 + [C-297, Russia: Primorskiy Kray, Vladivostok environs, ??. ??.1992, A. Okulov] (USNM).

SLOVENIA: 1 [Slov. Bohinj Bela, 5 Jun. 1979, P.H. and S.L. Ward] (handwritten) (BMNH B.M. 1979-263); 1 [Slovenia Postojne, 18 Jul. 1958, R.L. Coe, Wooded hill N.W. of town] (handwritten) (BMNH B.M. 1958-417); 1 q [Slovenia Postojne, 1 Aug. 1958, R.L. Coe, Wooded hill N.W. of town] (BMNH B.M. 1958-417).

SPAIN: 1 đ̊, 2 q $q$ [Madrid, Cercedilla, 22 Oct. 1978, J.S. Noyes] (BMNH B.M. 1978-488) (handwritten).

SWEDEN: 1 đ, 1 9 [sk. (Skåne) kivik, 19 Jul. 1938, d.m.s.p. (D.M.S. Perkins) and j.f.p. (J.F. Perkins)] (handwritten) (BMNH B.M. 1938-414); 1 \& [Sk., Silvakra, Stensoffa RN-1351/6176, T.H. and J.Q., Aug. 1976] (BMNH). 1 \& [Uppsala, Hogadalen, 16-10 Aug. 1990, Malaise trap, F. Ronquist] (CNCI C-307).

SWITZERLAND: 4 ふぇ, 4 우 [Dielsdorf, 650 m, 17 Aug. 1984, L. Masner, sweep] (CNCI C-290); 1 ㅇ [Turgau, Unterwasser environs, 1440 m, 4 Aug. 1984, L. Masner sweeping] (CNCI C-293).

UNITED KINGDOM: England: 1 [Surrey, Box Hill (near Dorking), 28 Aug. 1982, E.E. Grissell herbaceous vegetation] (USNM).

\section{Distribution}

Palaearctic, Oriental and Neotropical.

Certain records: Andorra (Ferrer-Suay et al. 2011: 240), Colombia (Ferrer-Suay et al. 2012f: 321), Corsica (Ferrer-Suay et al. 20131), France (Ferrer-Suay et al. 2015a), Italy (Ferrer-Suay et al. 2014b), Madeira (Ferrer-Suay et al. 2012g: 9), Mexico (Ferrer-Suay et al. 2013m: 30), Netherlands (Evenhuis, 1976: 143), Iran (Ferrer-Suay et al. 2013a: 32), Serbia and Slovenia (Ferrer-Suay et al. 2013b: 351), Spain (Kieffer 1902: 12), Taiwan and Thailand (Ferrer-Suay et al. 2013i).

Uncertain records: Romania (Ionescu 1969: 245, 268; Prelipcean et al. 2004: 60).

New records: Czech Republic, Egypt, England, France, Germany, Morocco, Poland, Russia, Sweden and Switzerland. 
Alloxysta aurata Belizin, 1968

Figs $2.5,4.4$

Alloxysta aurata Belizin, 1968: 716. Type: deposited in ZIN (examined).

\section{Diagnosis}

Alloxysta aurata is mainly characterized by a partially open radial cell that is 3.0 times as long as wide (Fig. 4.4); the presence of pronotal and propodeal carinae; and male and female antenna with rhinaria beginning on F4, F1 longer than F2, F2 shorter than F3, and F3 subequal to F4 (Fig. 2.5). It is similar to A. castanea, but can be differentiated by the $\mathrm{F} 2-\mathrm{F} 3$ proportion (F2 is shorter than $\mathrm{F} 3$ in A. aurata (Fig. 2.5), but subequal to $\mathrm{F} 3$ in $A$. castanea (Fig. 2.11)) and the size of the radial cell (3.0 times as long as wide in A. aurata (Fig. 4.4) but 2.4 times in A. castanea (Fig. 4.9)).

\section{Material examined}

\section{Holotype}

RUSSIA: + [Vladivostok, Akademgorodok, M. Kozlov, 9 Aug. 1961], [Holotypus Alloxysta aurata m

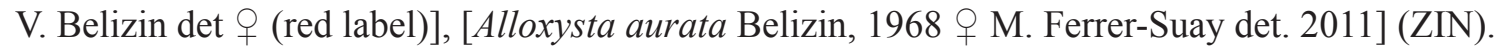

\section{Distribution}

Eastern Palaearctic.

Certain records: Siberia (Russia) (Belizin 1968: 716).

Alloxysta basimacula (Cameron, 1886)

Figs 2.6, 4.5

Allotria basimacula Cameron, 1886: 87. Type: deposited in BMNH (examined).

Dilyta basimacula - Kieffer 1900: 114.

Alloxysta (Alloxysta) basimacula - Dalla Torre \& Kieffer 1902: 38.

Alloxysta basimacula - Andrews 1978: 78.

\section{Diagnosis}

Alloxysta basimacula is mainly characterized by a completely open radial cell that is 3.0 times as long as wide (Fig. 4.5); the presence of pronotal carinae; the absence of propodeal carinae; and antenna with rhinaria beginning on F4, F1 longer than pedicel and subequal to F2, F2 longer than F3, and F3 shorter than F4 (Fig. 2.6). It is similar to A. brachycera, but can be differentiated by the F1-F2 proportion (F1 is subequal to F2 in A. basimacula (Fig. 2.6), but longer than F2 in A. brachycera (Fig. 2.7)) and the size of the radial cell (3.0 times as long as wide in A. basimacula (Fig. 4.5) but 2.7 times in A. brachycera (Fig. 4.6)).

\section{Material examined}

\section{Lectotype}

UNITED KINGDOM: Scotland: $q$ [Lectotype (round label with blue margin)], [Mugdock, Glasgow, basimacula] (handwritten), [Cameron 96 76. Mugdoch], [Lectotype + Allotria basimacula C. det. J. Quinlan, 1973] (handwritten), [B.M. TYPE HYM. 7.120], [Alloxysta basimacula (Cameron, 1886) det. M. Ferrer-Suay 2012] (BMNH B.M. Type Hym. 7.120). 
Additional specimens $(10$ q )

GERMANY: 1 [ [Schwarzwald, Todtmoos environs, 6 Aug. 1984, L. Masner, screen sweeping]; 4 q $q$ [C-235, Schwarzwald, Todtmoos environs, 6 Aug. 1984, L. Masner, screen sweeping] (CNCI C-235).

JAPAN: 2 우 [Hokkaido, Sapporo, Jozankei, 350 m, Aug. 1989, K. Maeto and M. Sharkey, MT] (CNCI

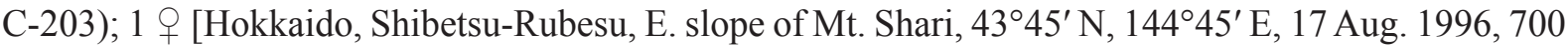
m, L. Masner, sweep] (CNCI C-164).

SWEDEN: 2 우 [Lappland, Riksgränsen, 500 m, 2 Aug. 1960, W.R.M. Mason] (CNCI C-311).

\section{Distribution}

Palaearctic.

Certain records: Scotland (Cameron 1886: 87-88; 1889: 58).

New records: Germany, Japan and Sweden.

Alloxysta barbotini Ferrer-Suay \& Pujade-Villar, 2016

Alloxysta barbotini Ferrer-Suay \& Pujade-Villar. Type: deposited in UB.

\section{Diagnosis}

Alloxysta barbotini is similar to Alloxysta australiae (Ashmead, 1900) because both species have a closed radial cell, pronotal and propodeal carinae present, and independent propodeal carinae. They can be differentiated by the antenna (rhinaria and club shape begin on F2 (female) or F1 (male) in A. barbotini, but on F4 in A. australiae (only female known); F2 is slightly longer than F3 and F3 is subequal to F4 in A. barbotini, but F2-F4 are subequal in length in A. australiae), propodeal carinae (wide and not defined posteriorly in A. barbotini but thin and straight in A. australiae), and the size of the radial cell (2.1 (female) and 2.0 (male) times as long as wide in A. barbotini but 2.4 times in A. australiae).

\section{Material examined}

\section{Holotype}

MOROCCO: + [Ifrane, 27 May 1977] (UB).

\section{Paratypes}

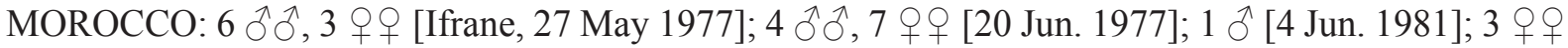

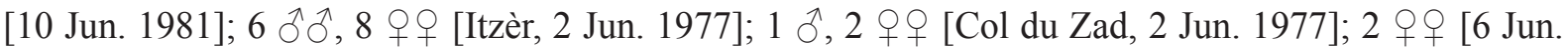
1981]; 4 우 [7 Jun. 1981]; 1 ô, 2 우 [9 Jun. 1981]; 1 ô, 2 우 [Aïn Kellah, 5 Jun. 1981] (UB).

\section{Distribution}

Palaearctic.

Certain records: Morocco (Ferrer-Suay et al. 2016).

\section{Alloxysta brachycera Hellén, 1963}

Figs 2.7, 4.6

Alloxysta brachycera Hellén, 1963: 14. Type: deposited in MZH (examined). 


\section{Diagnosis}

Alloxysta brachycera is mainly characterized by a completely open radial cell that is 2.7 times as long as wide (Fig. 4.6); the presence of pronotal carinae; the absence of propodeal carinae; and female antenna with rhinaria beginning on F4, F1 longer than pedicel and F2, F2 longer than F3, and F3 shorter than F4 (Fig. 2.7). It is similar to A. nigrita, but can be differentiated by the F2-F3 proportion (F2 is longer than F3 in A. brachycera (Fig. 2.7), but shorter than F3 in A. nigrita (Fig. 2.30)) and the size of the radial cell (2.7 times as long as wide in A. brachycera (Fig. 4.6), but 2.9 in A. nigrita (Fig. 5.2)).

\section{Material examined}

\section{Holotype}

FINLAND: + [Nystad, Hellén, 61, brachycera (handwritten)], [typus (red label)], [Mus. Zool. Helsinki, Loan No. HY 2012 - 1830 (yellow label)], [Alloxysta brachycera Hellén, 1963 q M. Ferrer-Suay det. 2012] (MZH).

\section{Distribution}

Europe.

Certain records: Finland (Hellén 1963: 14), France (Ferrer-Suay et al. 2015a).

Alloxysta brachyptera (Hartig, 1840)

Fig. 2.8

Xystus brachypterus Hartig, 1840: 200. Type: deposited in ZSM (examined).

Allotria brachyptera - Giraud 1860: 131.

Pezophycta brachyptera - Förster 1869: 339.

Pezophycta brachyptera brachyptera - Dalla Torre \& Kieffer 1910: 292.

Alloxysta brachyptera - Hellén 1931: 5.

\section{Diagnosis}

Alloxysta brachyptera is mainly characterized as a brachypterous species with pronotal carinae absent, propodeal carinae present, and F1 shorter than pedicel (Fig. 2.8). It is similar to A. pedestris, but can be easily differentiated by the presence of propodeal carinae (present in A. brachyptera but absent in A. pedestris).

\section{Material examined}

\section{Lectotype}

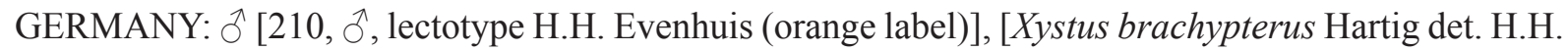
Evenhuis 1980], [Alloxysta brachyptera (Hartig, 1841) ô M. Ferrer-Suay det. 2011] (ZSM).

\section{Paralectotype}

GERMANY: 1 ठै [Paralectotype Xystus brachypterus Hartig, 1840 ${ }^{\lambda}$ (red label)], [Alloxysta brachyptera (Hartig, 1841) § M. Ferrer-Suay det. 2011] (ZSM).

\section{Additional specimens $(6 \hat{\jmath} \widehat{\jmath}, 3$ 우)}

FRANCE: 1 đ̊ [Pyrénées Orientales, Arles-Corsavy Road, D AND J Clark, 19 May 1961, A. Brachyptera Det. N.D.M. Fergusson, 19] (BMNH B.M. 1962-149). 
GERMANY: 2 つð, 2 우 [Göttingen, 29 Aug. 1959, H. Prilop Ex Dorilas fabae, C.I.E., coll. no. 16782] (BMNH).

JAPAN: $1 \lesssim$ [Kyushu, Fukuoka, Mt. Hiko, 700 m, 9-10 May 1989, M.J. Sharkey, sweep] (CNCI C-138).

NORWAY: $1 \hat{\jmath}$ [VE Tonsberg, Frodeasen, 19 Jul. 82, FJELOSA (handwritten)], [A. Brachyptera Det. N.D.M. Fergusson] (BMNH).

SWEDEN: 1 \& [SK. Kivik, 19 Jul. 1938, D.M.S.P. AND J.F.P.] (BMNH B.M. 1938-414).

SWITZERLAND: 1 ○े [Dielsdorf, 650 m, 17 Aug. 1984, L. Masner, sweep] (CNCI) C-290.

\section{Distribution}

Palaearctic.

Certain records: England (Dalla Torre \& Kieffer 1910: 292; Fergusson 1986: 18; Müller et al. 1999), Germany (Hartig 1840: 200).

Uncertain records: Austria (Giraud 1860: 131), Belgium (Lameere 1907), Finland (Hellén 1931: 5), France (Dalla Torre \& Kieffer 1910: 292), Ireland (Fergusson 1986: 18), Romania (Ionescu 1969: 236), Scotland (Fergusson 1986: 18), Sweden (Thomson 1862: 410).

New records: Japan, Norway and Switzerland.

Alloxysta brevis (Thomson, 1862)

Figs $2.9,4.7,8.2,10.2$

Allotria brevis Thomson, 1862: 408. Type: deposited in MZLU (examined).

Allotria (Allotria) brevis - Dalla Torre \& Kieffer 1902: 40.

Charips (Charips) brevis - Dalla Torre \& Kieffer 1910: 276.

Alloxysta brevis - Andrews 1978: 79.

\section{Diagnosis}

Alloxysta brevis is mainly characterized by a small closed radial cell that is 2.1 times as long as wide (Fig. 4.7); the absence of pronotal carina (Fig. 8.2); the presence of propodeal carinae that form a plate; and female and male antenna with rhinaria beginning on F4, F1 shorter than pedicel, and F1-F3 subequal in length (Fig. 2.9). It is similar to $A$. darci, but can be differentiated by the length of antenna (shorter than the body in A. brevis, but longer in $A$. darci) and the marginal setae of the fore wing (shorter in $A$. brevis than A. darci).

\section{Material examined}

\section{Lectotype}

SWEDEN: + [Ld (Lund) 8/6 (white label, handwritten)], [brevis (white label, handwritten)], [Lectotype Allotria brevis + Thomson, 1862 design. Ferrer-Suay and J.P-V 2011 (red label)], [Alloxysta brevis (Thomson) $\rightarrow$, M. Ferrer-Suay and J.P-V det.] (MZLU).

\section{Paralectotype}

SWEDEN: + [L-d (Lund) (white label)], [8-toma (white label, handwritten)], [1984/405 (green label, loan code)], [Paralectotype Allotria brevis $\bigcirc$ Thomson, 1862 (red label)], [Alloxysta brevis (Thomson) , M. Ferrer-Suay and J.P-V det. (white label)] (MZLU). 
Additional specimens $(13 \hat{\partial} \hat{\partial}, 135$ q $q)$

CROATIA: 1 + [Yugoslavia, Dalmatia, Drvenik nr. Makarska, 9.8.79, Boucek].

CZECH REPUBLIC: 1 [Moravia: Kobyli, Jul. 1970, M. Kocourek] (BMNH); 5 + $q$ [Wooden hill, N.W. of town, Moravia: Kobyli, Jul. 1970, M. Kocourek] (BMNH); 1 \& [Moravia, Zidlochovice, 20 Nov. 84, N.D. Springate] (BMNH); 1 q [central Bohemia, 30 Jul. 1991, J. Macek] (CNCI C-274); 1 ㅇ [central Bohemia, 30 Jul. 1991, J. Macek] (CNCI C-278); 1 ㅇ [Moravia, Lanzhot-Ranspurk, 7-9 Aug. 1991, L. Masner, climax flood forest] (CNCI C-265); 1 ô [Moravia, Tvrdonice, 7 Aug. 91, L. Masner, fraxinetum] (CNCI C-262); 1 \& [Praha-Troja, 6-9 Sep. 1999, YPT, L. Masner, steppe] (CNCI C-284).

FRANCE: 2 우 [Lot La Paylle Basse Sovillac, c 1200 ft, 28 May-1 Jun. 1979, R.S. George] (BMNH).

GERMANY: 1 ㅇ [Mainz, 18 Sep.-1 Oct. 1965, A.W. Steffan] (CNCI C-236); 1 q [Rhine Valley, Steinstadt, $47^{\circ} 46^{\prime}$ N, $07^{\circ} 34^{\prime}$ E, 7 Jun. 1999, H. Goulet, fallow field] (CNCI C-232); 1 \& [Rhine Valley near Hügelheim, 47 50'10" N, 7³7'9" E, 15 Jun. 1999, H. Goulet, sweeping old alfalfa] (CNCI C-230); 1 J , 3 우 [Schwarzwald, Todtmoos environs, 6 Aug. 1984, L. Masner, screen sweeping] (CNCI C-235).

GREECE: 2 q+ [Thessalia, Kalambaka (hillside meadow), 14-20 Jul. 1979, M.C. Day, G.R. Else, D. Morgan] (BMNH BM 1979-312); 1 \& [Greece: Ilia Olympia, 4-11 Jul. 1979, M.C. Day, G.R. Else, D. Morgan] (BMNH BM 1979-312).

HUNGARY: 1 q [Baranya Beremend, 24 Apr.-8 May 1963, L. Horacsek] (BMNH B.M. 1964-373).

IRAN: 3 우 [Karaj, 40 km W Tehran, 25-28 Jun. 1978, J.T. Huber, YPT] (CNCI C-316); 4 + $q$ [Karaj, 40 km W Tehran, 3 Jul. 1978, J.T. Huber sweep] (CNCI C-315).

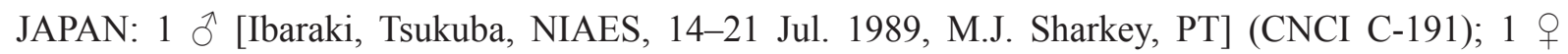
[Kyushu, 700 m, Fukuoka, MT. Hiko, 10-21 Jul. 1989, MT, K. Takeno and M. Sharkey] (CNCI C-171); 1 ठ [Aichi, 900 m, Shitara, Uradani, 20-26 Jun. 1994, K. Yamagishi, YPT, beech forest] (CNCI C-199); 1 ㅇ [Hokkaido, Aizan, 800 m, 4 Jul. 1989, sweep, M.J. Sharkey] (CNCI C-186); 1 ㅇ [Fukuoka, Mt. Hiko, 700 m, 9-10 May 1989, sweep, M.J. Sharkey] (CNCI C-183); 1 ठ [Ibaraki, Tsukuba, NIAES, 14-25 Jul. 1989, M. Sharkey, FIT and MT] (CNCI C-146); 1 ㅇ [Ibaraki, Tsukuba, NIAES, 1-3 Mar. 1990, M. Sharkey, FIT and MT] (CNCI C-149); 1 \& [Fukuoka, Mt. Hiko, 12-29 May 1989, Takeno and Sharkey] (CNCI C-145); 8 우 [Hokkaido, Sapporo, Jozankei, 350 m, 12-21 Sep. 1989, K. Maeto and M. Sharkey, MT] (CNCI C-205); 2 우 [Ibariki, Tsukuba, NIAES, 13 Nov.-22 Dec. 1989, M.J. Sharkey, PT] (CNCI C-142); 1 q [Aichi, Nagoya, Ogawa, 11 Jun. 1984, R. Baczyushi] (CNCI C-179); 2 우 [Hokkaido, Sapporo, Jozankei, 21-28 Sep. 1989, K. Maeto and M. Sharkey] (CNCI C-193); 1 ㅇ

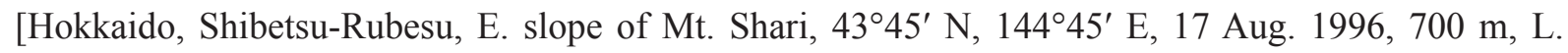
Masner, sweep] (CNCI C-164); 2 우 [Gifu, Kasagawa, Ikeda-cho, 24 May 1984, R. Baczyuski] (CNCI C-181); 1 q [Hokkaido, Sapporo, Jozankei, 350 m, 20-31 Jul. 1989, MT, K. Maeto and M. Sharkey] (CNCI C-204); 1 ठै [Aichi, Shitara, Uradani, 900 m, 11-17 Jul. 1994, K. Yamagishi, EMT, beech forest] (CNCI C-222); 10 우 [Hokkaido, Sapporo, Jozankei, 350 m, 10-21 Aug. 1989, K. Maeto and M. Sharkey, MT] (CNCI C-206); 1 \&Hokkaido, Nukabira, 600 m, 5 Jul. 1989, sweep, M.J. Sharkey] (CNCI C-215); 14 우 [Hokkaido, Sapporo, Jozankei, 350 m, 29 Aug.-12 Sep. 1989, K. Maeto and M. Sharkey, MT] (CNCI C-207).

MOROCCO: 1 ㅇ [Marrakech, Ouirgane, 1000 m, 31 $1^{\circ} 08^{\prime}$ N, $8^{\circ} 05^{\prime}$ W, 13-21 Mar. 1996, C. Kassebeer, MT] (CNCI C-280); 3 우우 [Marrakech, Ouirgane, $1000 \mathrm{~m}, 31^{\circ} 08^{\prime} \mathrm{N}, 8^{\circ} 05^{\prime} \mathrm{W}, 4-11$ Oct. 1996, C. Kassebeer, MT] (CNCI C-279). 
RUSSIA: 4 $q+$ [Russian Far East, Primorski Krai Lazovski Zapovednik, c.170Km, E. Vladivostok, Ta-Chingousa, $43^{\circ} 00^{\prime} 42^{\prime \prime}$ N, $134^{\circ} 07^{\prime} 34^{\prime \prime}$ E, Om 28 Aug.-16 Sep. 2001, sandy coast, Malaise trap 494', M. Quest coll. BMNH(E) 2009-59, Alloxysta spp-indet dwarfs and intermediates] (BMNH 200959); 15 우 [Primoskiy Kray Ussyriysk District, Gomotayozhnoye, 11-15 Aug. 2003, Malaise trap, M.V. Michailovskaya] (USNM); 1 q [Primorskiy Kray Ussyriysk District, Gomotayozhnoye, 200 m, $43.66^{\circ} \mathrm{N}, 132.25^{\circ} \mathrm{E}, 1-10$ Aug. 2002, M.V. Michailovskaya, MT] (USNM); 3 q $ᄋ$ [Primorskiy Kray, Vladivostok environs, ??. ??.1992, A. Okulov] (CNCI C-297).

SERBIA: 1 [Srbija Drazevac, nr. Belgrade, 27-28 Jun. 1981, M. Day and M. Fitton] (BMNH); 1 ㅇ [Srbija Drazevac, nr. Belgrade 27-28 Jun. 1981, M. Day and M. Fitton] (BMNH).

SPAIN: 1 ð̊, 2 우 [Madrid, Cercedilla, 22 Oct. 1978, J.S. Noyes, B.M. 1978-488] (BMNH).

SLOVAKIA: 1 q [C.S.S.R., Koš̀ice, 14-17 Sep. 84, N.D. Springate] (BMNH).

SLOVENIA: 3 q $q$ [Radovljica, 3 Aug. 1978, L. Huggert swept on dry meadow] (CNCI C-276); 4 $\widehat{\jmath}$, 6 우 [Slatna, Radovljica, 6 Aug. 1978, L. Huggert, swept on open wooded (deciduous) meadow] (CNCI C-275).

SWEDEN: 3 ㅇ [sk. kivik, 19 Jul. 1938, d.m.s.p. and j.f.p.] (BMNH B.M. 1938-414); 1 [ [Uppsala, Hogadalen, 16 Sep.-2 Oct. 1990, Malaise trap F. Ronquist] (CNCI C-304).

SWITZERLAND: 2 ふึ่, 15 우 [Dielsdorf, 650 m, 17 Aug. 1984, L. Masner, sweep] (CNCI C-290);

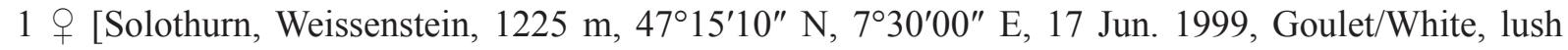
meadow] (CNCI C-285).

\section{Distribution}

Eastern Palaearctic and Holartic.

Certain records: Andorra (Ferrer-Suay et al. 2011: 350), Corsica (Ferrer-Suay et al. 20131: 5), England (Müller et al. 1999: 346), India (Ferrer-Suay et al. 2013i: 5), Iran (Ferrer-Suay et al. 2013a: 36), Italy (Ferrer-Suay et al. 2014e: 6), Mexico (Ferrer-Suay et al. 2013m: 32), Serbia and Slovenia (Ferrer-Suay et al. 2013b: 351), Sweden (Thomson 1862: 408), Thailand (Ferrer-Suay et al. 2013i: 5).

Uncertain records: Canada (Andrews 1978: 68), Finland (Hellén 1963: 22), France (Kieffer 1902b: 602; De Gaulle 1908: 26), Germany (Hübner et al. 2002: 507), Hawaii (Beardsley 1985: 50), Hungary (Fülöp et al. 2010: 54), Ireland (O’Connor \& Nash 1997), Japan (Takada \& Nakamura 2010: 269), Madeira (Borges et al. 2008), Poland (Barczak 1991: 87), Romania (Ionescu 1969: 245-246; Prelipcean et al. 2004: 60; Ceballos 1941: 226; Tizado \& Núñez-Pérez 1993: 97; Bertolaccini et al. 2004: 42), USA (California) (Oatman et al. 1983: 1714; Zuparko \& Dahlsten 1995: 730), USA (Florida) (Ashmead 1887: 19; Evans \& Stange 1997: 1), USA (Idaho) (Weld 1920: 15).

New records: Czech Republic, Greece, Moravia, Morocco, Russia, Spain, Switzerland.

Alloxysta brevitarsis (Thomson, 1862)

Figs 2.10, 4.8

Allotria brevitarsis Thomson, 1862: 409. Type: deposited in MZLU (examined).

Dilyta brevitarsis - Kieffer 1900: 114.

Alloxysta (Alloxysta) brevitarsis - Dalla Torre \& Kieffer 1902: 38.

Alloxysta brevitarsis - Hellén 1963: 14-15. 


\section{Diagnosis}

Alloxysta brevitarsis is mainly characterized by a closed radial cell that is 1.8 times as long as wide (Fig. 4.8); the presence of pronotal carinae; propodeal carinae that form a plate with very curved sides; and antenna with club shape beginning on F2, rhinaria beginning on F3, F1 longer than pedicel and F2, and F2 longer than F3 (Fig. 2.10). According to these features, there is no other species of Alloxysta similar to $A$. brevitarsis.

\section{Material examined}

\section{Lectotype}

SWEDEN: $\widehat{\alpha}$ [brevitarsis (gray label, handwritten)], [brevitarsis (handwritten)], [Lectotype Allotria brevitarsis Thomson, $1862 \precsim$ desig. M. Ferrer-Suay 2011 (red label)], [Alloxysta brevitarsis (Thomson, 1862) $ð$ M. Ferrer-Suay det. 2011, ZML.2011 061 (green label)] (MZLU).

\section{Distribution}

Europe.

Certain records: Sweden (Thomson 1862: 409).

Uncertain records: Finland (Hellén 1963: 15), France (Kieffer 1902b: 34; De Gaulle 1908: 26).

\section{Alloxysta castanea (Hartig, 1841)}

Figs 2.11, 4.9

Xystus castaneus Hartig, 1841: 352. Type: deposited in ZSM (examined).

Allotria castanea - Cameron 1890: 233.

Dilyta castanea - Kieffer 1900: 114.

Alloxysta (Alloxysta) castanea - Dalla Torre \& Kieffer 1902: 38.

Alloxysta castanea - Hellén 1963: 14.

\section{Diagnosis}

Alloxysta castanea is mainly characterized by a partially open radial cell that is 2.4 times as long as wide (Fig. 4.9); the presence of pronotal and propodeal carinae; and male and female antenna with rhinaria beginning on F3, F2-F4 subequal in length (Fig. 2.11), and slightly curved F1 and F2 in males. It is similar to A. aurata, but can be differentiated by the F2-F3 proportion (F2 is subequal to F3 in A. castanea (Fig. 2.11), but shorter than F3 in A. aurata (Fig. 2.5)) and the size of the radial cell (2.3 times as long as wide in A. castanea (Fig. 4.9), but 3.0 times in A. aurata (Fig. 4.4)).

\section{Material examined}

\section{Lectotype}

GERMANY: + [lectotypus Xystus castaneus Htg, Zoologische Staatssammlg. München (pink label)], [Lectotype design. Evenhuis, according to Evenhuis (1982) (red label)], [Alloxysta castanea (Hartig, 1841) ㅇ M. Ferrer-Suay det. 2011] (ZSM).

\section{Additional specimens $(89 \widehat{\jmath}, 287$ 우 $)$}

AUSTRIA: 1 [Tirol Kitzbuhel, 18-29 Aug. 62, A.H. Hayes, B.M. 1962-481]; 1 [Leibnitz, R. Mur, 3 Jun. 1969, B.H. and M.C. Cogan, R.I. and R. Vane-Wright] (BMNH B.M. 1970-152). 


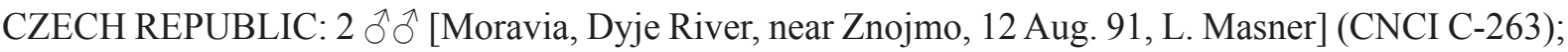

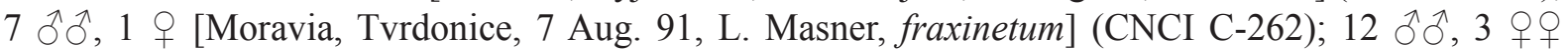
[Moravia, Lanzhot-Ranspurk, 7-9 Aug. 1991, L. Masner, sweep, climax flood forest] (CNCI C-268); 1 [ [Moravia, 16 km N Blansko, 8 Aug. 1991, L. Masner, sweep. Tilia-Acer forest] (CNCI C-271); 2 ऽో ${ }^{\lambda}, 3$ 우우 [Moravia, Lanzhot-Ranspurk, 7-9 Aug. 1991, L. Masner sweep, climax flood forest]

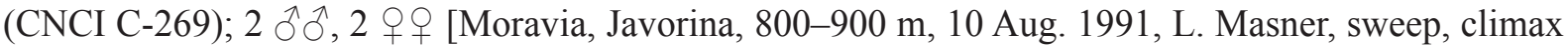
deciduous forest] (CNCI C-266); 1 § , 2 우 [Moravia, Ladnice environs 7-9 Aug. 1991, L. Masner riparian, forest] (CNCI C-272); 5 우 [Bohemia central, Řevnice, environs, YPT, 20-21 Aug. 1999, L. Masner, creek] (CNCI C-292); 3 우 우 [Moravia, Lanzhot-Ranspurk, 9-12 Aug. 1991, L. Masner, climax hardwood forest, PT] (CNCI C-270).

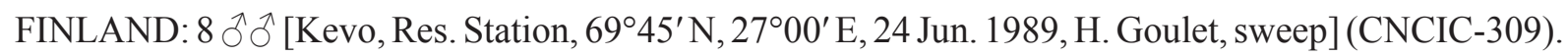

FRANCE: 2 $\precsim, 4 q q$ [Pyrénées Orientales, nr. Arles-sur-Tech, D. and J. Clark, 22 May 1961] (BMNH B.M. 1962-149); 1 + [Pyrénées Orientales, Arles-sur-Tech, D. and J. Clark, 25 Jun. 1961] (BMNH B.M. 1962-149); 2 우 [Vendée Longeville, 19-26 Sep. 1965, J.A.J. Clark] (BMNH B.M. 1965-489).

GERMANY: 3 우 [Mainz, 18 Sep.-1 Oct. 1965, A.W. Steffan] (CNCI C-236); 1 q [Mainz, 4-17 Sep. 1965, A.W. Steffan] (CNCI C-229); 2 우 [Ingelheim am Rhein, ??.??.1968, MT in orchard, A.W. Steffan] (CNCI C-238); 1 q [Ingelheim am Rhein, 1-30 Sep. 1968, MT, A.W. Steffan, orchard,

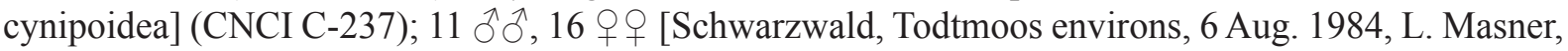
screen sweeping] (CNCI C-235).

HUNGARY: $1 \precsim$ [Baranya Beremend, 24 Apr.-9 May 1963, L. Horacsek] (BMNH B.M. 1964-373).

IRAN: 2 우 [Karaj, 40 km W Tehran, 3 Jul. 1978, J.T. Huber sweep] (CNCI C-315).

ITALY: $1 \hat{\partial}$ [Cortina D’Ampezzo, 24 Jul. 1969, M.C. Day, swept in grall] (BMNH).

JAPAN: 4 우 [Aichi Shitara, Uradani, $900 \mathrm{~m}$, beech forest, 23-29 May 1994, K. Yamagishi, YPT] (CNCI C-187); 1 9 [Kyushu, Fukuoka, Mt. Hiko, 700 m, 15 Nov.-1 Dec. 1989, K. Takeno, M. Sharkey, MT] (CNCI C-196); 1 9 [Kyushu, 700 m, Fukuoka, MT. Hiko, 10-21 Jul. 1989, MT, K. Takeno and M. Sharkey] (CNCI C-171); 4 우 ㅇ [Japan; Aichi, 900 m, Shitara, Uradani, 20-26 Jun. 1994, K. Yamagishi, YPT, beech forest] (CNCI C-199); 1 O̊, 2 오 [Hokkaido, 20 km N. Akkeshi, marsh, 100 m, 15 Jul. 1996, L. Masner, sweep] (CNCI C-160); 1 \& [Hokkaido, Aizan, 800 m, 4 Jul. 1989, sweep, M.J. Sharkey] (CNCI C-186); 1 q [Ibaraki, Tsukuba, NIAES, 22 Dec. 89-30 Jan. 1990, M. Sharkey, FIT and MT] (CNCI C-147); 1 [Kyushu, 700 m, Fukuoka, Mt. Hiko, 25 Jul.-4 Sep. 1989, MT, K. Takeno and M. Sharkey] (CNCI C-176); 1 §, 2 우 [Fukuoka, Mt. Hiko, 700 m, 9-10 May 1989, sweep, M.J. Sharkey] (CNCI C-183); 2 q + [Kyushu, 700 m, Fukuoka, Mt. Hiko, 9-19 Jun. 1989, MT, K. Takeno and M. Sharkey] (CNCI C-170); 1 [ [Ibaraki, Tsukuba, NIAES, 14-25 Jul. 1989, M. Sharkey, FIT and MT] (CNCI C-146); 2 우 [Fukuoka, Mt. Tachibana, 18-28 Jun. 1979, K. Yamagishi, PT] (CNCI C-143); 1 q [Ibaraki, Tsukuba, Expo site, 11-18 Sep. 1989, M.J. Sharkey, PT] (CNCI C-134); 2 q $q$ [Kyushu, Fukuoka, Mt. Hiko, 700 m, 18-25 Aug. 1989, MT, K. Takeno and M. Sharkey] (CNCI C-185); 1 ㅇ [Hokkaido, Sapporo, Jozankei, 10-21 Aug. 1989, 350 m, K. Maeto and M. Sharkey, MT] (CNCI C-197); 2 우 [Aichi, Mt. Chausu (SSW), 1300 m, 9 Jul. 1995, K. Yamagishi] (CNCI C-156); 6 우 [Hokkaido, Sapporo, Jozankei, 350 m, 20-31 Jul. 1989, K. Maeto and M. Sharkey] (CNCI C-151); 2 우 [Hokkaido, Sapporo, Jozankei, 350 m, Aug. 1989, K. Maeto and M. Sharkey, MT] (CNCI C-203); 2 우우 [Hokkaido, Sapporo, Jozankei, 350 m, 30 Jun.-11 Jul. 89, MT, K. Maeto and M. Sharkey] (CNCI C-184); 1 + [Ibaraki, Tsuchiura City, Shishizuka-oike, 13-25 Jul. 1989, MT, M.J. Sharkey, marsh] (CNCI C-175); 1 ㅇ [Aichi, Shitara, Uradani, 900 m, 18-24 Jul. 1994, K. Yamagishi, MT, beech forest] (CNCI C-167); 1 \& [Honshu, Iwata, Mt. Hayachine, 400 m, 11-19 Jul. 1989, H. Makihara and M. Sharkey, MT] 
(CNCI C-177); 1 q [Hokkaido, Sapporo, Jozankei, 21-28 Sep. 1989, 350 m, K. Maeto and M. Sharkey MT] (CNCI C-158); 1 q [Ibaraki, Tsukuba, Expo site, 27 Oct-3 Nov. 1989, M.J. Sharkey, PT] (CNCI C-133); 1 q [Ibariki, Tsuchiura, Shishizaka-oike, 15-21 Aug. 1989, M. Sharkey, marsh] (CNCI C-141); 1 을 [Aichi, Shitara, Uradani, 900 m, 21 May 1994, K. Yamagishi, PT, beech forest] (CNCI C-155); 1 [Fukuoka, Mt. Hiko, 25 Aug-4 Sep. 1989, Takeno and Sharkey] (CNCI C-144); 2 q 9 [Ibaraki, Tsukuba, NIAES, 1-3 Mar. 1990, M. Sharkey, FIT and MT] (CNCI C-149); 1 ô, 1 ㅇ [Fukuoka, Mt. Hiko, 12-29 May 1989, Takeno and Sharkey] (CNCI C-145); 3 우 우 [Hokkaido, Sapporo, Jozankei, 350 m, 12-21 Sep. 1989, K. Maeto and M. Sharkey, MT] (CNCI C-205); 1 q [Fukuoka, Mt. Hiko, 700 m, 28 Apr.-10 May 1989, M.J. Sharkey, MT] (CNCI C-139); 1 ð̊, 4 우 [Kyushu, 700 m, Fukuoka, MT. Hiko, 21-29 Jul. 1989, MT, K. Takeno and M. Sharkey] (CNCI C-172); 1 [Gifu, Mt. Ena-san (ssw), 1200-1900 m, 5 Jun. 1995, K. Yamagishi] (CNCI C-189); 1 [Hokkaido, Shibetsu-Rubesu,

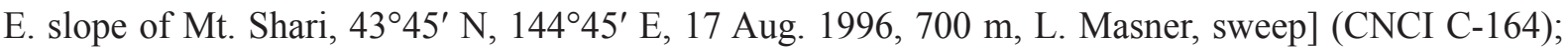
5 우 우 [Aichi, Shitara, Uradani, 900 m, 29 Jun.-3 Jul. 1994, K. Yamagishi, YPT, beech forest] (CNCI C-188); 2 q 9 [Kyushu, Fukuoka, Mt. Hiko, 700 m, 11-13 Aug. 1989, MT, K. Takeno and M. Sharkey] (CNCI C-169); 1 ㅇ [Ibaraki, Tsukuba, Expo site, 7-16 May 1989, M.J. Sharkey, PT] (CNCI C-135); 5 우 [Kyushu, Fukuoka, Mt. Hiko, 700 m, 19-29 Jun. 1989, MT, K. Takeno and M. Sharkey] (CNCI C-174); 1 ㅇ [Hokkaido, Sapporo, Jozankei, 350 m, 21-29 Aug. 1989, K. Maeto and M. Sharkey, MT] (CNCI C-202); 1 [Japan; Hokkaido, Sapporo, Jozankei, 350 m, 20-31 Jul. 1989, MT, K. Maeto and M. Sharkey] (CNCI C-204); 1 ô [Aichi, Toyone, 1300 m, Mt. Chausu, 16 Jul. 1992, K. Yamagishi, sweep] (CNCI C-132); 1 q [Ibaraki, Tsukuba, NIAES, 26 Apr.-2 May 1989, M.J. Sharkey, PT] (CNCI C-190); 1 q [Hokkaido, Sapporo, Jozankei, 350 m, 20-31 Jul. 1989, M. Sharkey, K. Maeto, MT] (CNCI C-137); 1 ㅇ [Ibaraki, Mt. Tsukuba, 800 m, 2-26 Oct. 1989, M.J. Sharkey, PT] (CNCI C-168); 2 우 [Aichi, Shitara, Uradani, 900 m, 9-15 May 1994, K. Yamagishi, EMT, beech forest] (CNCI C-218); 3 우 [Aichi, Shitara, Uradani, 900 m, 30 May-5 Jun. 1994, K. Yamagishi, EMT, beech forest] (CNCI C-220); 1 q [Mainz, 4-17 Sep. 1965, A.W. Stefan] (CNCI C-229); 1 đ [Aichi, Shitara, Uradani, 900 m, 30 May-5 Jun. 1994, K. Yamagishi, EMT, beech forest ] (CNCI C-216); 12 우아 [Hokkaido, Sapporo, Jozankei, 350 m, 10-21 Aug. 1989, K. Maeto and M. Sharkey, MT] (CNCI C-206); 1 q [Aichi, Shitara, Uradani, 900 m, 2-8 May 1994, K. Yamagishi, EMT, beech forest] (CNCI C-224); 1 q [Hokkaido, Sapporo, Jozankei, 350 m, 20-31 Jul. 1989, K. Maeto and M. Sharkey] (CNCI C-209); 2 우 [Hokkaido, Nukabira, 600 m, 5 Jul. 1989, sweep, M.J. Sharkey] (CNCI C-215); 2 우 [Aichi, Shitara, Uradani, 900 m, 4-10 Jul. 1994, K. Yamagishi, EMT, beech forest] (CNCI C-217); 7 q 9 [Aichi, Shitara, Uradani, 900 m, 6-12 Jun. 1994, K. Yamagishi, EMT, beech forest] (CNCI C-221); 3 우 [Hokkaido, Sapporo, Jozankei, 350 m, 29 Aug.-12 Sep. 1989, K. Maeto and M. Sharkey, MT] (CNCI C-207); 5 우 [Honshu, Tochigi, Kuriyama, betw. Meatobuchi and Koniyu, 1420 m, 20-23 Aug. 1991, A. Smetana (J63)] (CNCI C-211); 3 우 [Kyushu, Fukuoka, Mt. Tachibana 20 May-9 Jun. 1979, K. Yamagishi YPT, cutover land] (CNCI C-213).

F.Y.R. MACEDONIA: $1 \hat{\jmath}$ [Verge of oak wood above lake, Yugoslavia, Macedonia, prespa Geril, 22 Jun. 1958, R.L. Coe.] (BMNH B.M. 1958-417).

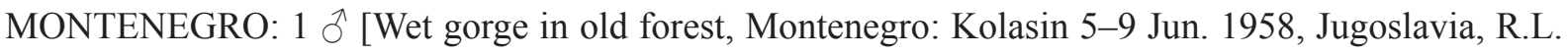
Coe] (BMNH B.M. 1958-417).

NETHERLANDS: 1 [ [Wageningen I.P.O boom-gaard, 7-8-1961 (handwritten), verzameld van pruim (handwritten), mummie: Hyalopterus pruni det. H.R.L, Alloxysta sp. क det. J. Quinlan 1962] (BMNH); 1 ภ, 2 우 [Wageningen, Netherlands, 24 Aug. 1977, leg. H.H. Evenhuis (handwritten), aphid mummy on leaf of Phragmites australis (handwritten), Alloxysta ruficollis (Cameron) det. H.H. Evenhuis 1979] (BMNH); 1 ð, 2 우 [Wageningen, Netherlands, 24 Aug. 1977, leg. H.H. Evenhuis (handwritten), aphid mummy on Centaurea protensis UACO (handwritten), Alloxysta defecta (Hartig) det. H.H. Evenhuis 1981] (BMNH); $1 \hat{\jmath}$ [Langbrook (U.), Netherlands 13 Aug. 1978, leg. H.H. Evenhuis (handwritten), 
aphid mummy on Phragmites australis (handwritten), Alloxysta castanea (Hartig) det. H.H. Evenhuis 1981] (BMNH); 1 q [Bennekon De Meet, Netherlands leg. H.H. Evenhuis (handwritten), aphid mummy on Centaurea sp. 9-7-1975 (handwritten), Alloxysta defecta (Hartig det.) H.H. Evenhuis 1989] (BMNH).

RUSSIA: 3 우 [Russian Far East, Primorskii Krai Lazovski Zapovednik, c. 170 Km E., Vladivostok,

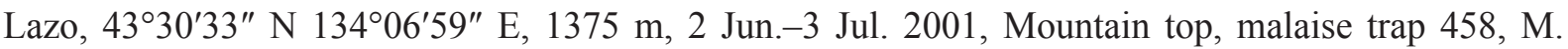
Quest coll. BMNH(E) 2009-59, Alloxysta sp1., det. M. Forshage 2012] (BMNH B.M. 2009-59); 1 ㅇ [Russian Far East, Primorski Krai Lazovski Zapovednik, c.170 Km, E. Vladivostok, Ta-Chingousa,

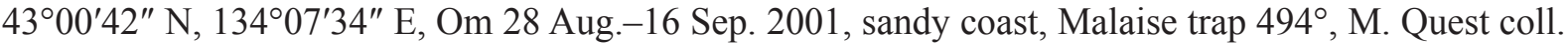
BMNH(E) 2009-59, Alloxysta sp 4, det. M. Forshage 2012] (BMNH B.M. 2009-59); 2 우 [Russian Far East, Primorski Krai Lazovski Zapovednik, c. $170 \mathrm{Km}$, E. Vladivostok, Ta-Chingousa, $43^{\circ} 00^{\prime} 42^{\prime \prime} \mathrm{N}$,

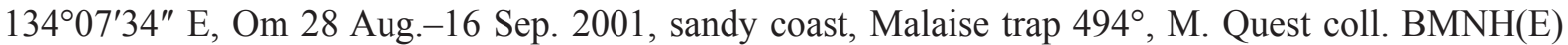
2009-59, Alloxysta spp-indet dwarfs and intermediates] (BMNH B.M. 2009-59); 7 우 [Primoskiy Kray Ussyriysk District, Gomotayozhnoye, 11-15 Aug. 2003, Malaise trap, M.V. Michailovskaya] (USNM); 4 ̂ํㅅ, 45 우우 [Primoskiy Kray Ussyriysk District, Gomotayozhnoye, 11-15 Aug. 2003, Malaise trap M.V. Michailovskaya] (USNM); 1 \& [Primorskiy Kray Ussyriysk District, Gomotayozhnoye, 200 m, $43.66^{\circ}$ N, $132.25^{\circ}$ E, 1-10 Aug. 2002, M.V. Michailovskaya, MT] (USNM); 3 q $q$ [Krasnodor Prov., Temnolesskaya, 15 May-15 Jun. 1999, V. Grebennikov, YPT] (CNCI C-294); 3 우 우 [Primorskiy Kray, Vladivostok environs, ??. ??.1992, A. Okulov] (CNCI C-297).

SLOVAKIA: 1 [ [Koš̀ice, 14-17 Sep. 84, N.D. Springate] (BMNH); 4 우 [Čachtice environs, 2 Aug. 1991, L. Masner, sweep] (CNCI C-267).

SLOVENIA: 4 우 [Slovenia Postojne, 18 Jul. 1958, R.L. Coe Wooded hill N.W. of town] (BMNH

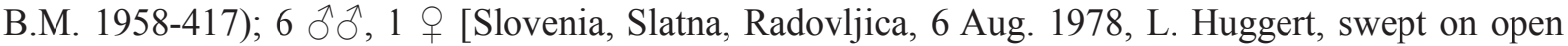
wooded (deciduous) meadow] (CNCI C-275); 1 q [Slovenia, Radovljica, 3 Aug. 1978, L. Huggert swept on dry meadow] (CNCI C-276).

SWEDEN: 1 q [Sk. Lund Zoological Mus. grounds, Aug. 1976: 1 क; So. Malmen Dalaro, 15-26 Aug. 1976, J.Q. and T.H] (BMNH); 2 우 [Sk., Lund Zoological Mus. grounds, Aug. 1976] (BMNH); 1 ○, 1 [Sk., Rolsberga Rovurekulan RN-1354/6188, T.H. and J.Q., Aug. 1976] (BMNH); 1 ㅇ [Sk., Silvakra Stensoffa, RN-1351/6176, T.H. and J.Q., Aug. 1976, A. fulviceps det. N.D.M. Fergusson] (BMNH); 1 ㅇ [Stockholm, Nora, 20 Aug. 1933, G.J. Kerrich] (BMNH B.M. 1969-261); 1 đ, 3 우 [Sk. Fjellfota sjö, 7 Aug. 1938, D.M.S.P. and J.F.P.] (BMNH B.M. 1938-414); 1 ㅇ [Up. Solna Bergshamra, 15-26 Aug. 1976, J.Q. and T.H.] (BMNH); 1 o [Uppsala, Hogadalen, 10-17 Aug. 1990, Malaise trap, F. Ronquist] (CNCI C-305); 1 q [Lappland, Riksgränsen, 500 m, 2 Aug. 1960, W.R.M. Mason] (CNCI C-311); 1 त [Kalmar, Överum, 19 May-29 Jun. 1978, L. Huggert, MT at swampy lake shore] (CNCI C-312).

SWITZERLAND: 1 ㅇ [Zürich W. Dill 1934 (handwritten), Ex. Hyalopterus anuudinis (= pruni), Prunus sp, Charips cameroni D.T. o, Ch. Ferriere det] (BMNH); 1 o [Zürich W. Dill 1934 (handwritten), Ex Hyalopterus anuudinis (= pruni) Prunus sp. (handwritten), Pres by Imp. Inst. Ent., Charips cameroni

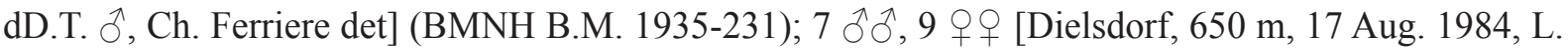
Masner, sweep] (CNCI C-290); 1 q [Jura, Delémont, CABI Lab. 47²2'42" N, 7²0'30" E, 10-19 Jun.

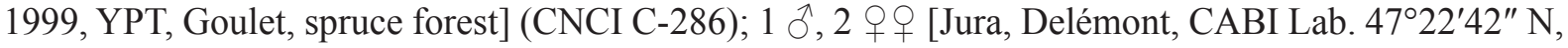
7²0'30" E, 7 Jun. 1999, H. Goulet, spruce/beech] (CNCI C-287); 5 §ิㅅ, 5 우우 [Dielsdorf, $650 \mathrm{~m}$, 17 Aug. 1984, L. Masner, sweep] (CNCI C-290); 1 đ [Turgau, Unterwasser environs, 1440 m, 4 Aug. 1984, L. Masner sweeping] (CNCI C-293).

\section{Distribution}

Palaearctic and Neotropical. 
Certain records: Colombia (Ferrer-Suay et al. 2012f: 322), Corsica (Ferrer-Suay et al. 20131), Germany (Hartig 1841: 352), Iran (Ferrer-Suay et al. 2013a: 36), Italy (Ferrer-Suay et al. 2014b), Madeira (FerrerSuay et al. 2012g: 10), Mexico (Ferrer-Suay et al. 2013m: 32), Montenegro, Serbia and Slovenia (FerrerSuay et al. 2013b: 354), Japan, S. Korea, Malaysia, Nepal and Taiwan (Ferrer-Suay et al. 2013i).

Uncertain records: Austria (Kieffer 1902b: 35), Denmark (Andrews 1978: 82), England (Cameron 1889: 54; Dalla Torre \& Kieffer 1910: 256), Finland (Hellén 1963: 14), France (Kieffer 1902a: 10, 15; 1902b: 595; De Gaulle 1908: 26; Cavro 1954: 12), Germany (Hedicke 1928: 94; Hübner et al. 2002: 507), Kuriles Island (Belizin 1962: 128), Norway (Hofsvang \& Hagvar 1983: 60), Romania (Ionescu 1969: 266; Prelipcean et al. 2004: 60), Scotland (Cameron 1883: 365), The Netherlands (Andrews 1978: 82), USA (Colorado) (Baker 1896: 133; Andrews 1978: 52).

New records: Czech Republic, Germany, Hungary, Italy, Japan, Russia, Sweden, Switzerland.

Alloxysta circumscripta (Hartig, 1841)

Figs 2.12, 4.10

Xystus circumscriptus Hartig, 1841: 352. Type: deposited in ZSM (examined).

Allotria circumscripta - Giraud 1860: 127.

Allotria (Allotria) circumscripta - Dalla Torre \& Kieffer 1902: 40.

Charips (Charips) circumscripta - Dalla Torre \& Kieffer 1910: 277.

Alloxysta circumscripta - Hellén 1963: 17.

\section{Diagnosis}

Alloxysta circumscripta is mainly characterized by a closed radial cell that is 2.3 times as long as wide (Fig. 4.10); the presence of pronotal carinae; the absence of propodeal carinae; female antenna with rhinaria beginning on F5, F2 shorter than F3, and F3 shorter than F4 (Fig. 2.12); male antenna with rhinaria beginning on F4, F2 longer than F3, and F3 shorter than F4. It is similar to A. consobrina, but can be differentiated by the flagellomere proportions (F1 is subequal to F2 and F2 is shorter or subequal to F3 in A. circumscripta (Fig. 2.12), while F1 is longer than F2 and F2 is subequal to F3 in A. consobrina (Fig. 2.14)) and the size of the radial cell (2.5 times as long as wide in A. circumscripta (Fig. 4.10), but 2.7 in A. consobrina (Fig. 4.12)).

\section{Material examined}

Lectotype

GERMANY: + , [lectotype H.H. Evenhuis (orange label)], [Xystus circumscriptus Hartig det. H.H. Evenhuis 1980], [Alloxysta circumscripta (Hartig, 1841) ㅇ M. Ferrer-Suay det. 2011] (ZSM).

\section{Paralectotype}

GERMANY: 1 [ [n collection Hartig as Xystus circumscriptus], [Paralectotype Xystus circumscriptus Hartig, 1841 ( (red label)], [Alloxysta circumscripta (Hartig, 1841) + M. Ferrer-Suay det. 2011] (ZSM).

\section{Distribution}

Europe.

Certain records: Andorra (Ferrer-Suay et al. 2011: 354), England (Müller et al. 1999: 346), Germany (Hartig 1841: 352). 
Uncertain records: Austria (Giraud 1860: 127; Hellén 1963: 17), England (Dalla Torre \& Kieffer 1910: 278), Finland (Hellén 1963: 17), France (De Gaulle 1908: 26), Poland (Kierych 1979b: 15), Scotland (Cameron 1886: 86).

Alloxysta citripes (Thomson, 1862)

Figs 2.13, 4.11

Allotria citripes Thomson, 1862: 410. Type: deposited in MZLU (examined).

Alloxysta citripes var britannica Kieffer, 1902: 11. Synonymized by Evenhuis (1976: 140). Type: BMNH (Evenhuis 1976: 140).

Dilyta citripes - Kieffer 1900: 114.

Alloxysta (Alloxysta) citripes - Dalla Torre \& Kieffer 1902: 38.

Alloxysta (Alloxysta) citripes var britannica - Dalla Torre \& Kieffer 1902: 38.

Alloxysta (Alloxysta) citripes citripes - Dalla Torre \& Kieffer 1910: 261.

Alloxysta (Alloxysta) citripes britannica - Dalla Torre \& Kieffer 1910: 262.

Alloxysta citripes citripes - Andrews 1978: 80.

\section{Diagnosis}

Alloxysta citripes is mainly characterized by a partially open small radial cell that is 2.1 times as long as wide (Fig. 4.11); the presence of pronotal carinae; propodeal carinae that form a plate but are not protruding; female antenna with rhinaria beginning on F4, F1 subequal to pedicel and longer than F2, and F2-F4 subequal in length (Fig. 2.13); male antenna with rhinaria beginning on F1, pedicel-F3 subequal, and F3 slightly shorter than F4. It is similar to A. postica, but can be differentiated by the shape of propodeal carinae (they do not protrude in A. citripes, while they are clearly visible and form a protruding plate in A. postica) and the size of the radial cell (2.1 times as long as wide in A. citripes (Fig. 4.11), but 2.5 times in A. postica (Fig. 5.9)).

\section{Material examined}

Lectotype

SWEDEN: o [1972, 38 (green label)], [lectotype H.H. Evenhuis (orange label)], [Alloxysta citripes (Thomson) det. H.H. Evenhuis 1976], [1984, 407 (green label)], [ZML. 2011071 (green label)] (MZLU).

Additional specimens $(2 \hat{\partial} \widehat{\partial}, 15$ q + )

CROATIA: 1 \& [Yugoslavia, Dalm Isl. Mljet N.P., 6 Sep. 1980, Boucek] (BMNH).

CZECH REPUBLIC: 1 [Moravia, Palava near Mikulov, $\approx 400$ m, 9 Aug. 91, L. Masner, sweep] (CNCI C-264); 1 + [Moravia, Javorina, 800-900 m, 10 Aug. 1991, L. Masner, sweep, climax deciduous forest] (CNCI C-266).

FRANCE: 1 q [B. du Rhone Fonscolombe (3), 2 Jul. 1980, M.W.R. de V. Graham coll. BMNH(E)] (BMNH).

GERMANY: 1 [ [Schwarzwald, Todtmoos environs, 6 Aug. 1984, L. Masner, screen sweeping] (CNCI C-235).

IRAN: 1 ð [IKaraj, 40 km W Tehran, 25-28 Jun. 1978, J.T. Huber, YPT] (CNCI C-316).

JAPAN: $1 \hat{\jmath}$ [Hokkaido, Sapporo, Jozankei, 350 m, 20-31 Jul. 1989, K. Maeto and M. Sharkey] (CNCI C-151); 1 \& [Hokkaido, Sapporo, Jozankei, 350 m, 10-21 Aug. 1989, K. Maeto and M. Sharkey, MT] 
(CNCI C-206); 3 우 [Hokkaido, Sapporo, Jozankei, 350 m, 29 Aug.-12 Sep. 1989, K. Maeto and M. Sharkey, MT] (CNCI C-207).

SWEDEN: 1 [Sk. Silvakia, Stensoffa, RN-1351/6176, T.H. and J.Q. Aug. 1976] (BMNH); 1 q [Uppsala, Hogadalen, 26 Aug.--5 Sep. 1990, Malaise trap, F. Ronquist] (CNCI C-306).

SWITZERLAND: 3 우 [Jura, Delémont, CABI Lab. 47²2'42" N 7²0'30" E, 7 Jun. 1999, H. Goulet, spruce/beech] (CNCI C-287).

UNITED KINGDOM: England: 1 [ [Surrey Chobham Common, 26 Aug. 1982, E.E. Grissell sweeping oak, Cynipidae] (USNM).

\section{Distribution}

Palaearctic.

Certain records: Andorra (Ferrer-Suay et al. 2011: 355), Corsica (Ferrer-Suay et al. 20131), England (Kieffer 1902a: 11; Fergusson 1986: 18), Iran (Rakhshani et al. 2001: 42; 2004: 3), Italy (Ferrer-Suay et al. 2014b), Portugal (Ferrer-Suay et al. 2012i: 10), The Netherlands (Evenhuis 1976: 140), Sweden (Thomson 1862: 410).

Uncertain records: France (Kieffer 1902b: 600; De Gaulle 1908: 26), Germany (Hübner et al. 2002: 508), Hungary (Fülöp et al. 2010: 55), Moldova (Belizin 1966: 6), Scotland (Cameron 1886: 87).

New records: Czech Republic, Germany, Japan and Switzerland.

Alloxysta consobrina (Zetterstedt, 1838)

Figs 2.14, 4.12

Cynips consobrina Zetterstedt, 1838: 410. Type: deposited in MZLU (examined).

Xystus fuscicornis Hartig, 1841: 352. Synonymized by Ferrer-Suay et al. (2013a: 86). Type: ZSM (examined).

Allotria consobrina - Dahlbom 1842: 4.

Allotria (Allotria) consobrina - Dalla Torre \& Kieffer 1902: 41.

Charips (Charips) consobrinus - Dalla Torre \& Kieffer 1910: 288.

Alloxysta consobrina - Forshage in Ferrer-Suay et al. 2012a: 22.

\section{Diagnosis}

Alloxysta consobrina is mainly characterized by a closed radial cell that is 2.7 times as long as wide (Fig. 4.12); the presence of pronotal carinae; the absence of propodeal carinae; and male and female antenna with rhinaria beginning on F4, F2 longer than F3, F3 shorter than F4 (Fig. 2.14), and bowed F1-F3 in males. It is similar to A. circumscripta, but can be differentiated by flagellomere proportions (F1 is longer than $\mathrm{F} 2$ and $\mathrm{F} 2$ is subequal to $\mathrm{F} 3$ in $A$. consobrina (Fig. 2.14), while $\mathrm{F} 1$ is subequal to $\mathrm{F} 2$ and F2 is shorter than or subequal to F3 in A. circumscripta (Fig. 2.12)) and the size of the radial cell (2.7 times as long as wide in A. consobrina (Fig. 4.12), but 2.5 times in A. circumscripta (Fig. 4.10)).

\section{Material examined}

\section{Lectotype}

LAPPLAND (no more detailed information): + [lectotypus Xystus castaneus Htg, Zoologische Staatssammlg. München (pink label)], [Lectotype design. Evenhuis, according to Evenhuis (1982) (red label)], [Alloxysta castanea (Hartig, 1841) o M. Ferrer-Suay det. 2011] (MZLU). 
Additional specimens $(22 \hat{\jmath}, 175$ 우 $)$

AUSTRIA: 2 우 우 [Tyrol nr. Oetz., 8oo-900 m, 5-14 Jun. 1964, J.A.J. Clark] (BMNH B.M. 1965-494).

SERBIA: 1 ㅇ [Beograd 23 May 1962, swept on Lucerne, N. Tanasijevic per V.F. Eastop 87] (BMNH); 1 ठ [Beograd, 23 May 1962, swept on Lucerne, N. Tanasijevic per V.F. Eastop. 89] (BMNH).

CZECH REPUBLIC: 1 đ 1 1 [Moravia, Lanzhot-Ranspurk, 7-9 Aug. 1991, L. Masner, sweep, climax flood forest] (CNCI C-268); 2 우 [Moravia, Javorina, 800-900 m, 10 Aug. 1991, L. Masner, sweep, climax deciduous forest] (CNCI C-266).

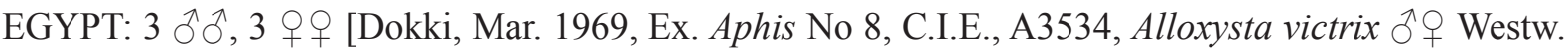
det. J. Quinlan, 1970] (BMNH); 1 ㄱ, 3 우우 [Ex Brevicoryne brassicae L. (handwritten), Egypt, Giza, 8 Aug. 1964, D. Herakly (handwritten), Alloxysta (Först) Hellén? minuta Htg ô, det. J. Quinlan, 1965] (BMNH); 2 ふ઼่, 4 우 [Beheira, 15 Apr. 1975, M. El-DoKroury] (BMNH).

GERMANY: 1 ㅇ [Mainz, 18 Sep.-1 Oct. 1965, A.W. Steffan]; 1 ㅇ [C-228, Hesse, Gelnhausen, Sep. 1967, MT, A.W. Steffan] (CNCI C-236); 2 수, 16 우우 [Schwarzwald, Todtmoos environs, 6 Aug. 1984, L. Masner, screen sweeping] (CNCI C-235).

JAPAN: 1 [Kyushu, Fukuoka, Mt. Hiko, 700 m, 9-10 May 1989, M.J. Sharkey Sweep] (CNCI C-138); 1 q [Ibaraki, Tsukuba, NIAES, 10-17 Jul. 1989, M. Sharkey, FIT and MT] (CNCI C-148); 4 우 [Fukuoka, Mt. Hiko, 700 m, 9-10 May 1989, sweep, M.J. Sharkey] (CNCI C-183); 2 우 [Ibaraki, Tsukuba, NIAES, 8-15 Jun. 1989, M. Sharkey, FIT and MT] (CNCI C-150); 2 우 [Ibaraki, Tsukuba, NIAES, 27 Oct.-13 Nov. 1989, PT, M. Sharkey] (CNCI C-200); 3 우우 [Fukuoka, Mt. Hiko, 12-29 May 1989, Takeno and Sharke] (CNCI C-145); 2 우 [Ibaraki, Tsukuba, Expo site, 7-16 May 1989, M.J. Sharkey, PT] (CNCI C-135).

MALTA: 1 [May 1982, sp DI C.I.E. A. 13986, Alloxysta Först? sp. $q$ det. J. Quinlan, 1982] (BMNH).

PALESTINE: 7 ふึ, 10 우 [Mikwe, 16 Feb. 1947, per Chief Plant Protection Officer Ex Cabbage Aphis, Com. Inst. Ent. Coll. No, 10803, Pres. by Com. Inst. Ent.] (BMNH B.M. 1948-321); 1 o, 1 o [Mikwe, 16 Jul. 1947, C2, per Chief Prant, Protection Officer, Ex Cabbage Aphis, Com. Inst. Ent. Coll. No. 10803, Pres. by Com. Inst. Ent.] (BMNH B.M. 1948-321).

RUSSIA: 1 [ [Primoskiy Kray Ussyriysk District, Gomotayozhnoye, 11-15 Aug. 2003, Malaise trap, M.V. Michailovskaya] USNM); 1 \& [Primorskiy Kray Ussyriysk District, Gomotayozhnoye, $43.66^{\circ} \mathrm{N}$, 132.25 E, el. 200 m, 10-19 Jul. 2002, M.V. Michailovskaya, MT] (USNM)

SLOVENIA: 1 ๙ [Slov. Bohinj Bela, 5 Jun. 1979, P.H. and S.L. Ward] (BMNH B.M. 1979-263).

SWEDEN: 1 [Sk. Lund Zoological Mus. grounds Aug.1976: 3 $\precsim$ $\partial$ and 18 $q$ $q$; Sö., Sandemar T.H. and

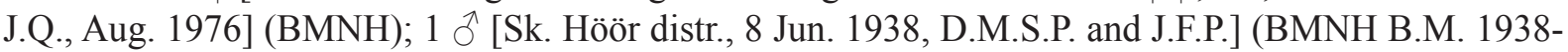
414); 2 우 [Sk, S. Sandby Maryd, T.H. and J.Q. Aug.1976] (BMNH); 4 우 [Sk., Lund Zoological Mus, grounds, Aug. 1976] (BMNH).

SWITZERLAND: 26 $ぇ ึ, 33$ 우 [Dielsdorf, 650 m, 17 Aug. 1984, L. Masner, sweep] (CNCI C-290); 51 우 [Turgau, Unterwasser environs, 1440 m, 4 Aug. 1984, L. Masner sweeping] (CNCI C-293).

SYRIA: 1 [hebanon aley, 14 Jan. 1941, A.S. Iachauk, FA Carly lower aphis (handwritten), Charips sp. G. Nixon det. 1944] (BMNH).

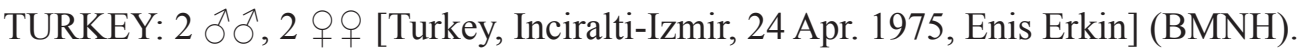




\section{Distribution}

Cosmopolitan.

Certain records: Africa (no country mentioned) (Evenhuis 1974: 167), Andorra (Ferrer-Suay et al. 2011: 356), Argentina (de Santis 1937: 1; Pujade-Villar et al. 2002: 543; Berta et al. 2002: 67), Brazil (Maria de Sousa \& Paes Bueno 1993-1994: 29; Betini 1975: 54, 1976: 76; Lazzari 1985: 9; Teixeira 1991: 47; Cividanes 2002: 251; Pujade-Villar et al. 2002: 543; Vaz et al. 2004: 225), Chile (Pujade-Villar et al. 2002: 543), Colombia (Pujade-Villar et al. 2010b), England (Müller et al. 1999: 352; van Veen et al. 2003: 450), Germany (Hartig 1841: 352; van Veen et al. 2003: 450; Hübner et al. 2002: 508), Italy (Ferrer-Suay et al. 2014b), Lappland (Zetterstedt 1838: 410), Mexico (Ferrer-Suay et al. 2013m: 35), New Zealand (Valentine 1975: 59; Ferrer-Suay et al. 2012h: 232), Peru (Pujade-Villar et al. 2002: 543), Serbia (Ferrer-Suay et al. 2013b: 356), Uruguay (Pujade-Villar et al. 2002: 543), Spain (Archimowitsch 1952: 112), India (Ferrer-Suay et al. 2013i).

Uncertain records: Australia (Froggatt 1904: 603; Carver 1992: 775), France (Kieffer 1902a: 16; De Gaulle 1908: 26), Hawaii (Beardsley 1985: 50), Hungary (Dalla Torre \& Kieffer 1910: 285), Iran (Lotfalizadeh 2002b: 1; Lotfalizadeh \& van Veen 2004: 119), Ireland (Chua 1978: 436), Romania (Feraru et al. 2005: 67), Scandinavia (Zetterstedt 1838: 410), Scotland (Cameron 1886: 85), Tenerife (Kieffer 1904: 63), USA (California) (Horn 1988: 354), USA (Florida) (Ashmead 1887: 9; Spencer 1926: 148), Wales (Weld 1952: 252).

New records: Austria, Czech Republic, Egypt, Japan, Malta, Palestine, Russia, Syria, Switzerland and Turkey.

Alloxysta curta Ferrer-Suay, 2017

Alloxysta curta Ferrer-Suay, 2017: 425. Type: deposited in UB.

\section{Diagnosis}

Alloxysta curta is characterized as a brachypterous species very similar to A. glebaria, with a small closed radial cell. However, these two species can be differentiated by the presence of pronotal carinae (present in A. curta but absent in A. glebaria).

\section{Material examined}

\section{Holotype}

SPAIN: đ [4 Jun. 1996, Lleonart (Argentona), greenhouse, Macrosiphum euphorbiae on Solanum lycopersicum], [Holotype Alloxysta curta Ferrer-Suay đ̃, Design. M. Ferrer-Suay, 2014], [Alloxysta curta Ferrer-Suay đ̊ M. Ferrer-Suay det. 2014] (UB).

\section{Paratypes}

SPAIN: $5 \widehat{\partial} \widehat{\partial}$ [26/06/1995, Lleonart (Argentona), greenhouse on Solanum lycopersicum; Paratype Alloxysta curta Ferrer-Suay ô;; Alloxysta curta Ferrer-Suay ổ M. Ferrer-Suay det. 2014] (UB).

\section{Distribution}

Spain (Ferrer-Suay et al. 2017). 


\section{Alloxysta crassa (Cameron, 1889)}

Figs 2.15, 4.13

Allotria crassa Cameron, 1889: 59. Type: deposited in BMNH (examined).

Dilyta crassa - Kieffer 1900: 114.

Alloxysta (Alloxysta) crassa - Dalla Torre \& Kieffer 1902: 38.

Alloxysta crassa - Hellén 1963: 12.

\section{Diagnosis}

Alloxysta crassa is mainly characterized by a completely open radial cell that is 2.8 times as long as wide (Fig. 4.13); the presence of pronotal carinae; the absence of propodeal carinae; antenna with rhinaria and club shape beginning on F4, F1 longer than pedicel and slightly longer than F2, and F2-F4 subequal in length (Fig. 2.15). It is similar to A. brachycera, but can be differentiated by the F2-F3 proportion (F2 is subequal to $\mathrm{F} 3$ in $A$. crassa, while $\mathrm{F} 2$ is longer than $\mathrm{F} 3$ in A. brachycera).

\section{Material examined}

\section{Lectotype}

UNITED KINGDOM: Scotland: क [Lectotype (round label with blue margins)], [Bonar (handwritten)], [crassa Cam (handwritten)], [Cameron 96 76, Bonar Bridge, Sutherland, Scotland], [Lectotype of Allotria crassa Cam. + det. J. Quinlan, 1973], [B.M. Type Hym. 7.128], [Alloxysta crassa (Cameron, 1889) ㅇ det. M. Ferrer-Suay 2012] (BMNH B.M. Type Hym. 7.128).

\section{Additional specimens (14 $\circ$ )}

FRANCE: 1 q [Pyrénées Orientales, Arles-Corsavij road, D. and J. Clark, 19 May 1961] (BMNH B.M. 1962-149).

GERMANY: 2 q $q$ [Mainz, 15 Nov. 1965, A.W. Steffan] (CNCI C-233).

ITALY: 2 우 [Dolomites, Seis am Scjleru. 1-13 Jun. 1964: 1 울 [Scily Is., Tresco, 2/9/75, J. Noyes] $(\mathrm{BMNH})$.

SLOVENIA: 1 q [Slovenia, Slatna, Radovljica, 6 Aug. 1978, L. Huggert, swept on open wooded (deciduous) meadow] (CNCI C-275).

SWEDEN: 3 우 [Sk. Lund, Zoological Mus. grounds, Aug. 1976] (BMNH); 2 우 [Sk. Röstanga, 6 Jul. 1938, D.M.S.P. and J.F.P.] (BMNH B.M. 1938-414); 1 ㅇ [Sk. Silvakia Stensoffa, RN-1351/6176, T.H. and J.Q. Aug. 1976] (BMNH).

SWITZERLAND: 1 [ [Dielsdorf, 650 m, 17 Aug. 1984, L. Masner, sweep] (CNCI C-290).

\section{Distribution}

Europe.

Certain records: Scotland (Cameron 1889: 59).

New records: France, Germany, Italy, Slovenia, Sweden and Switzerland.

Alloxysta crassicornis (Thomson, 1862)

Figs 2.16, 4.14

Allotria crassicornis Thomson, 1862: 407. Type: deposited in MZLU (examined). 
Allotria (Allotria) crassicornis - Dalla Torre \& Kieffer 1902: 40.

Charips (Charips) crassicornis - Dalla Torre \& Kieffer 1910: 281.

Alloxysta crassicornis - Hellén 1963: 19.

\section{Diagnosis}

Alloxysta crassicornis is mainly characterized by a closed radial cell that is 2.6 times as long as wide (Fig. 4.14); the presence of pronotal carinae; propodeal carinae that form a plate with slightly curved margins; and antenna with rhinaria and club shape beginning on F3, F1 subequal to pedicel and slightly longer than F2, and F2-F4 subequal in length (Fig. 2.16). According to these features, A. crassicornis is very similar to $A$. arcuata, but can be differentiated by flagellomere proportions (F2-F4 are subequal in length in A. crassicornis (Fig. 2.16), while $\mathrm{F} 2$ is subequal to $\mathrm{F} 3$ and $\mathrm{F} 3$ is shorter than $\mathrm{F} 4$ in $A$. arcuata (Fig. 2.4)) and the size of the radial cell (2.6 times as long as wide in A. crassicornis (Fig. 4.14), but 2.3 times in A. arcuata (Fig. 4.3)).

\section{Material examined}

\section{Lectotype}

SWEDEN: + [Esperörd 12 lund. 38.; 89.; क̂ं; ㅇ; crassicornis T-2 (gray label, handwritten)], [crassicornis (handwritten)], [Lectotype Allotria crassicornis Thomson, 1862 ㅇ desig. M. Ferrer-Suay 2011 (red label)], [Alloxysta crassicornis (Thomson, 1862) ㅇ M. Ferrer-Suay det. 2011], [ZML.2011 062 (green label)] (MZLU).

\section{Distribution}

Europe.

Certain records: Sweden (Thomson 1862: 407).

Uncertain records: Finland (Hellén 1963: 20), France (Kieffer 1902b: 599; De Gaulle 1908: 26), Romania (Ionescu 1969: 260).

\section{Alloxysta darci (Girault, 1933)}

Fig. 10.3

Allotria d'arci Girault, 1933: 2. Type: deposited in QM (examined).

Charips d'arci-Weld 1952: 252.

Alloxysta d'arci-Andrews 1978: 81.

Alloxysta darci - Carver 1992: 777.

\section{Diagnosis}

Alloxysta darci is mainly characterized by a small closed radial cell; the absence of pronotal carinae; the presence of a propodeal plate; antenna with rhinaria and club shape beginning on F4; F1 shorter than pedicel; and antenna longer than body length in both males and females. This species is similar to A. brevis, but can be differentiated by the length of antenna (longer than the body in $A$. darci, but shorter in A. brevis), and the marginal setae on the fore wing (longer in A. darci than in A. brevis).

\section{Material examined}

\section{Holotype}

AUSTRALIA: + [Allotria d'arci Girault $q$ type (handwritten, in front)], [Forest, Wynnum (handwritten, behind)], [Charips d'arci (Gir.) EF Riek det 1953], [Alloxysta darci (Girault) Mary Carver det.1992] (QM). 


\section{Distribution}

Palaearctic and Australian region.

Certain records: Australia (Girault 1933: 2; Ferrer-Suay et al. 2012a), Spain (Ferrer-Suay et al. 2013g: 324).

Alloxysta fracticornis (Thomson, 1862)

Figs 2.17, 4.15

Allotria fracticornis Thomson, 1862: 408. Type: deposited in MZLU (examined).

Allotria (Allotria) fracticornis - Dalla Torre \& Kieffer 1902: 40.

Charips (Charips) fracticornis - Dalla Torre \& Kieffer 1910: 281.

Alloxysta fracticornis - Andrews 1978: 83.

\section{Diagnosis}

Alloxysta fracticornis is mainly characterized by a closed radial cell that is 2.2 times as long as wide (Fig. 4.15); the absence of pronotal carinae; the presence of propodeal carinae; and male and female antenna with rhinaria beginning on F3, F1-F3 subequal in length (Fig. 2.17), and curved F3 in males. It is similar to A. mullensis, but can be differentiated by the F1-pedicel proportion (F1 is longer than pedicel in A. fracticornis (Fig. 2.17), but subequal to pedicel in A. mullensis (Fig. 2.28)), flagellomere proportions (F1-F3 are subequal in length in A. fracticornis (Fig. 2.17), but F1 is longer than F2 and $\mathrm{F} 2$ is subequal to $\mathrm{F} 3$ in A. mullensis (Fig. 2.28)), and flagellomere shape (F3 is curved in A. fracticornis males, but no flagellomeres are curved in A. mullensis males).

\section{Material examined}

\section{Lectotype}

SWEDEN: $\widehat{\partial}$ [fracticornis (gray label, handwritten)], [fracticornis (handwritten)], [Lectotype Allotria fracticornis Thomson, $1862 \AA$ desig. M. Ferrer-Suay 2011 (red label)], [Alloxysta fracticornis (Thomson, 1862) §ै M. Ferrer-Suay det. 2011], [ZML.2011 063 (green label)] (MZLU).

\section{Additional specimens $(27 \hat{\jmath} \hat{\partial}, 39 \stackrel{+}{\phi})$}

AUSTRIA: 2 우 [Austria: Tirol Obergurgl, 2000 m, Jul. 81, Day and Fitton: 1 q; Iran: Damavand, 40 Km E. Tehran, Jul.-Sep. 1978 M. Cox] (BMNH).

CZECH REPUBLIC: 1 § [Moravia: Kobyli, Jul. 1970, M. Kocourek] (BMNH).

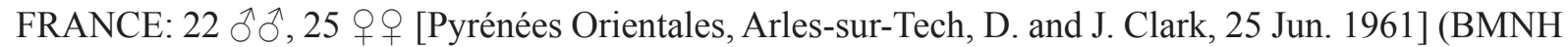
B.M. 1962-149); 1 ठิ [Vendée Longeville, 19-26 Sep. 1965, J.A.J. Clark] (BMNH B.M. 1965-489); 1 ô [B. du Rhone Fonscolombe (3), 2 Jul. 1980, M.W.R. de V. Graham coll.] (BMNH B.M. 1995-489).

IRAN: 1 [ [Damavand, $40 \mathrm{~km}$ E. Tehran in Orchard, 7-15 Jul. 1977, M. Cox] (BMNH); 1 q [Mazandoran province: Dauravand, 40 Km E of Tehran, Jul.-Sep. 1978: M. Cox] (BMNH B.M. 2007-70); 1 ๙ [Tehran Prov., Shahdasht, 25 Jun. 1978, J.T. Huber] (CNCI C-317).

JAPAN: 1 [Fukuoka, Mt. Hiko, 700 m, 9-10 May 1989, sweep, M.J. Sharkey] (CNCI C-183).

RUSSIA: 2 $q$ [Russian Far East, Primorski Krai Lazovski Zapovednik, c.170Km, E. Vladivostok,

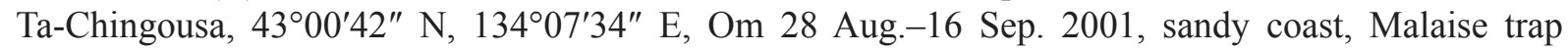
494², M. Quest coll., Alloxysta sp4, det. M. Forshage 2012] (BMNH B.M. 2009-59); 1 q [Russian Far 
East, Primorski Krai Lazovski Zapovednik, c.170 Km, E. Vladivostok, Ta-Chingousa, 4300'42" N, $134^{\circ} 07^{\prime} 34^{\prime \prime}$ E, Om 28 Aug.-16 Sep. 2001, sandy coast, Malaise trap 494², M. Quest coll., Alloxysta sppindet dwarfs and intermediates] (BMNH B.M. 2009-59).

SLOVAKIA: 1 q [C.R.R.R., Koš̀ice, 14-17 Sep. 84, N.D. Springate] (BMNH).

SLOVENIA: 1 [Rupa, 28 Apr. 1975, J.S.N., Grasland]; 1 \& [Slov. Viata Valley, 13 Jun. 1979, P.H. and S.L. Ward] (BMNH B.M. 1979-263).

SPAIN: 2 우 [Madrid, Cercedilla, 22 Oct. 1978, J.S. Noyes] (BMNH B.M. 1978-488).

SWITZERLAND: 1 ○ [Dielsdorf, 650 m, 17 Aug. 1984, L. Masner, sweep] (CNCI C-290).

\section{Distribution}

Palaearctic.

Certain records: France (Ferrer-Suay et al. 2015a), Italy (Ferrer-Suay et al. 2014b), Spain (Ferrer-Suay et al. 2013g), Sweden (Thomson 1862: 408).

Uncertain records: Austria (Andrews 1978: 83), Poland (Kierych 1979b: 14), Romania (Ionescu 1969: 251).

New records: Iran, Japan, Russia and Switzerland.

Alloxysta fuscipes (Thomson, 1862)

Figs 2.18, 4.16

Allotria fuscipes Thomson, 1862: 410. Type: deposited in MZLU (examined).

Dilyta fuscipes - Kieffer 1900: 114.

Alloxysta (Alloxysta) fuscipes - Dalla Torre \& Kieffer 1902: 38.

Alloxysta fuscipes - Hellén 1931: 4.

\section{Diagnosis}

Alloxysta fuscipes is characterized by a partially open radial cell that is 2.9 times as long as wide (Fig. 4.16); the presence of pronotal carinae; the absence of propodeal carinae; antenna with rhinaria and club shape beginning on F4; female antenna with F1 longer than pedicel and shorter than F2 and F2-F4 subequal in length (Fig. 2.18); male antenna with curved F2 and F3, F1 longer than pedicel and shorter than F2, F2 longer than F3, and F3 longer than F4. No other known species of Alloxysta closely resembles A. fuscipes.

\section{Material examined}

\section{Lectotype}

SWEDEN: $q$ [L-d; fuscipes (handwritten)], [1872, 40], [Lectotype Allotria fuscipes Thomson, 1862 q desig. M. Ferrer-Suay 2011 (red label)], [Alloxysta fuscipes (Thomson, 1862) $\odot$ M. Ferrer-Suay det. 2011], [ZML. 2011064 (green label)] (MZLU).

\section{Paralectotypes}

SWEDEN: 1 ㅇ [Lund 1972, 39], [Paralectotype Allotria fuscipes Thomson, 1862 q (red label)], [Alloxysta fuscipes (Thomson, 1862) ㅇ M. Ferrer-Suay det. 2011]; 1 q [Råby, 8/9. L (handwritten)], 
[1972, 41], [Paralectotype Allotria fuscipes Thomson, 1862 + (red label)], [Alloxysta fuscipes (Thomson, 1862) \& M. Ferrer-Suay det. 2011] (MZLU).

Additional specimens $(1 \hat{\delta}, 3$ 우)

ICELAND: 1 \%, 3 우 [Ex. Cavariella archangelicae (Scop.) (handwritten), Iceland, Reykjavik, 20 Jul. 1975, J. Couchman (handwritten), Alloxysta fuscipes (Thomson) det. H.H. Evenhuis, 1976] (BMNH).

\section{Distribution}

Europe.

Certain records: Serbia and Slovenia (Ferrer-Suay et al. 2013b: 355), Sweden (Thomson 1862: 410).

Uncertain records: Austria (Hellén 1963: 13), England (Andrews 1978: 83), Finland (Hellén 1931: 4; 1963: 13), Iceland (Hellén 1931: 4), (Hellén 1963: 13), Norway (Hellén 1966: 393), Russia (Hellén 1963: 13), Scotland (Cameron 1886: 88).

\section{Alloxysta glebaria Hellén, 1963}

Fig. 2.19

Alloxysta glebaria Hellén, 1963: 22. Type: deposited in MZH (examined).

\section{Diagnosis}

Alloxysta glebaria is mainly characterized as a brachypterous species with a radial cell present. According to these features, $A$. glebaria is very similar to $A$. marshalliana, but can be easily differentiated by the shape of the radial cell (closed in A. glebaria but completely open in A. marshalliana).

\section{Material examined}

\section{Holotype}

FINLAND: [Hammarland; Hellén; 4622], [Typus Alloxysta glebaria Hellén (handwritten) (red label)], [Sp2 not understandable (handwritten)], [glebaria (handwritten)], [Mus. Zool. Helsinki, Loan No. HY 2012 -1827 (yellow label)], [Alloxysta glebaria Hellén, 1963 + M. Ferrer-Suay det. 2012] (MZH).

\section{Distribution}

Europe.

Certain records: Finland (Hellén 1963: 22).

\section{Alloxysta halterata (Thomson, 1862)}

Fig. 2.20

Allotria halterata Thomson, 1862: 410. Type: deposited in MZLU (examined).

Pezophycta halterata - Kieffer 1900: 114.

Alloxysta halterata - Hellén 1963: 20.

\section{Diagnosis}

Alloxysta halterata is easily differentiated from the other brachypterous species of Alloxysta (A. brachyptera, A. pedestris and A. apteroidea) as it has pronotal carinae while the others do not. 
Females with normal sized wings are similar to A. victrix because both species have a closed radial cell and no propodeal carinae, but they can be differentiated by the size of the radial cell (2.4 times as long as wide in some A. halterata females but 3.0 times in A. victrix (Fig. 5.21)) and the F1-F2 proportion in females ( $\mathrm{F} 1$ is subequal to $\mathrm{F} 2$ in $A$. halterata, while $\mathrm{F} 1$ is longer than $\mathrm{F} 2$ in $A$. victrix (Fig. 3.17)).

\section{Material examined}

\section{Lectotype}

SWEDEN: $\widehat{\partial}$ [1969, 97 (green label)], [1984, 408 (green label)], [sintype A. halterata Thomson, det. N.D.M. Fergusson, 1984], [ZML 2000, 002 (green label)], [Lectotype Allotria halterata Thomson, 1862 $\lesssim$ desig. M. Ferrer-Suay 2011 (red label)], [Alloxysta halterata (Thomson, 1862) § M. Ferrer-Suay det. 2011] (MZLU).

\section{Additional specimens $(3 \hat{\jmath}, 2$ q + )}

FINLAND: 1 [Helsingfors, 12.9.1933, G.J. Kerrich, BM 1969, 261, A. pedestris N.D.M. Fergusson,

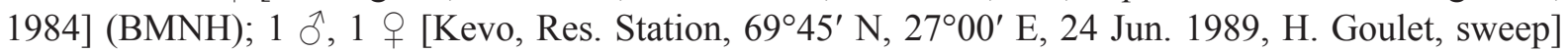
(CNCI C-309).

FRANCE: 1 ○े [Pyrenees Orientales Nr. MontFerrer, 19 Jun. 1962, J.A.J. Clark, BM (red label)] (BMNH B.M. 1962-342).

SLOVENIA: 1 ○ [Slovenia, Slatna, Radovljica, 6 Aug. 1978, L. Huggert, swept on open wooded (deciduous) meadow] (CNCI C-275).

\section{Distribution}

Europe.

Certain records: England (Müller et al. 1999: 346), Madeira (Ferrer-Suay et al. 2012g: 11), Sweden (Thomson 1862: 410).

Uncertain records: England (Hellén 1963: 20), Finland (Hellén 1963: 20), Germany (Hübner et al. 2002: 507), Romania (Ionescu 1969: 233), Scotland (Cameron 1886: 88).

New record: France.

Alloxysta heptatoma Hellén, 1963

Fig. 2.21

Alloxysta heptatoma Hellén, 1963: 22. Type: deposited in MZH (examined).

\section{Diagnosis}

Alloxysta heptatoma is mainly characterized by a small closed radial cell; the presence of pronotal carinae; well-defined propodeal carinae with sharp edges; and antenna with rhinaria and club shape beginning on F5, F1 shorter than pedicel, and F1-F3 subequal in length (Fig. 2.21). There is no other known Alloxysta species closely similar to A. heptatoma.

\section{Material examined}

\section{Holotype}

FINLAND: 9 [Dragsfjärd; Fennia; Hellén; 4032; heptatoma m(handwritten)], [aperta Hg (handwritten)], [typus Alloxysta heptatoma Hellén (handwritten) (red label)], [Mus. Zool. Helsinki, Loan No. HY 20121828 (yellow label)], [Alloxysta heptatoma Hellén, 1963 q M. Ferrer-Suay det. 2012] (MZH). 
Additional specimens $(2$ 우)

RUSSIA: 2 우 [Primoskiy Kray Ussyriysk District, Gomotayozhnoye, 11-15 Aug. 2003, Malaise trap, M.V. Michailovskaya] (USNM).

\section{Distribution}

Europe.

Certain records: Finland (Hellén 1963: 22).

Uncertain recods: Germany (Hübner et al. 2002: 507).

New record: Russia.

Alloxysta kovilovica Ferrer-Suay \& Pujade-Villar, 2013

Figs 2.22, 4.18

Alloxysta kovilovica Ferrer-Suay et al., 2013b: 256. Type: deposited in UB (examined).

\section{Diagnosis}

Alloxysta kovilovica is mainly characterized by a closed radial cell that is 2.5 times as long as wide; the absence of pronotal and propodeal carinae; and antenna with club shape and rhinaria beginning on F3, F1 longer than pedicel and F2, F2 equal to F3, and F3 slightly shorter than F4. No other known species of Alloxysta is closely related.

\section{Material examined}

\section{Holotype}

SERBIA: $q$ [14 Jun. 2010, alfalfa field, complex landscape, Kovilovo, Serbia, Alloxysta brevis (Thomson) q det. A. Stojanović. 2012] (UB).

\section{Additional specimens $(1$ )}

CROATIA: 1 \& [Istria Rusnjak, 1 May 1975, J.S. and M.E. Noyes, B.M. 1975-208] (BMNH).

\section{Distribution}

Europe.

Certain records: Serbia (Ferrer-Suay et al. 2013b: 256).

\section{Alloxysta leunisii (Hartig, 1841)}

Figs 2.23, 4.19

Xystus leunisii Hartig, 1841: 351. Type: deposited in ZSM (examined).

Allotria leunisii - Taschenberg 1866: 129.

Allotria (Allotria) leunisii - Dalla Torre \& Kieffer 1902: 40.

Charips (Charips) leunisii - Dalla Torre \& Kieffer 1910: 275.

Alloxysta leunisii-Andrews 1978: 84. 


\section{Diagnosis}

Alloxysta leunisii is mainly characterized by a closed radial cell that is 2.0 times as long as wide (Fig. 4.19), the presence of pronotal carinae, the absence of propodeal carinae, antenna with rhinaria and club shape beginning on F2 in females and F3 in males, female antenna with F1 longer than pedicel and subequal to F2 and F3 (Fig. 2.23), and male antenna with the same proportions as females except that F3 is longer than F2. It is similar to A. consobrina, but can be differentiated by the place where rhinaria begin in females (F2 in A. leunisii (Fig. 2.23) and F3/F4 in A. consobrina (Fig. 2.14)), flagellomere shape in males (F1 is slightly curved in A. leunisii while F1-F3 are strongly curved in A. consobrina), and the size of the radial cell (2.0 times as long as wide in A. leunisii (Fig. 4.19) but 2.7 times in A. consobrina (Fig. 4.12)).

\section{Material examined}

\section{Lectotype}

GERMANY: [ [?], [lectotype H.H. Evenhuis (orange label)], [Xystus leunisii Hartig det. H.H. Evenhuis 1980], [Alloxysta leunisii (Hartig, 1841) + M. Ferrer-Suay det. 2011] (ZSM).

\section{Paralectotypes}

GERMANY: 4 우 [In collection Hartig as Xystus leunisii; Paralectotype Xystus leunisii Hartig, 1841 (red label)], [Alloxysta leunisii (Hartig, 1841) q M. Ferrer-Suay det. 2011] (ZSM).

Additional specimens $(43 \hat{\jmath}, 16$ 우)

GERMANY: 7 우 [Schwarzwald, Todtmoos environs, 6 Aug. 1984, L. Masner, screen sweeping] (CNCI C-235).

SWITZERLAND: 43 $\widehat{\jmath}, 8$ 우 [Dielsdorf, 650 m, 17 Aug. 1984, L. Masner, sweep] (CNCI C-290); 1 [Turgau, Unterwasser environs, 1440 m, 4 Aug. 1984, L. Masner, sweeping] (CNCI C-293).

\section{Distribution}

Europe.

Certain records: England (van Veen et al. 2003: 450), Germany (Hartig 1841: 351; Höller et al. 1993: $13)$.

Uncertain records: Romania (Feraru et al. 2005: 67).

New record: Switzerland.

Alloxysta longipennis (Hartig, 1841)

Figs 2.24, 4.20

Xystus longipennis Hartig, 1841: 352. Type: deposited in ZSM (examined).

Allotria longipennis - Taschenberg 1866: 130.

Dilyta longipennis - Kieffer 1900: 114.

Alloxysta (Alloxysta) longipennis - Dalla Torre \& Kieffer 1902: 38.

Alloxysta longipennis - Andrews 1978: 85.

\section{Diagnosis}

Alloxysta longipennis is mainly characterized by a partially open radial cell that is 2.6 times as long as wide (Fig. 4.20); the presence of pronotal and propodeal carinae that form a plate with straight sides; and 
female antenna with rhinaria beginning on F3, F1 longer than pedicel and F2, F2 subequal to F3, and F3 shorter than F4 (Fig. 2.24). Male characteristics are unknown. It is similar to A. melanogaster, but can be differentiated by flagellomere proportions in females (F1 is longer than pedicel in A. longipennis (Fig. 2.24), while $\mathrm{F} 1$ is subequal to pedicel in A. melanogaster (Fig. 2.27)) and the size of the radial cell (2.6 times as long as wide in A. longipennnis (Fig. 4.20) but 2.3 times in A. melanogaster (Fig. 4.23)).

\section{Material examined}

Lectotype

GERMANY: $q$ [1149], [], [lectotype H.H. Evenhuis (orange label)], [Xystus longipennis Hartig det. H.H. Evenhuis 1980], [Alloxysta longipennis (Hartig, 1841) ㅇ M. Ferrer-Suay det. 2011] (ZSM).

\section{Distribution}

Europe.

Certain records: Germany (Hartig 1841: 352).

\section{Alloxysta macrophadna (Hartig, 1841)}

Figs 2.25, 4.21

Xystus macrophadnus Hartig, 1841: 352. Type: deposited in ZSM (examined).

Allotria macrophadna - Giraud 1860: 130.

Alloxysta macrophadna - Förster 1869: 340.

Allotria macrophadnus - Cameron 1889: 55.

Dilyta macrophadnus - Kieffer 1900: 114.

Alloxysta (Alloxysta) macrophadna - Dalla Torre \& Kieffer 1902: 38.

Alloxysta macrophadnus - Rohwer \& Fagan 1919: 340.

Charips macrophadnus - Muesebeck \& Krombein 1951: 607.

Alloxysta macrophadna-Andrews 1978: 85.

\section{Diagnosis}

Alloxysta macrophadna is mainly characterized by a big, partially open radial cell (Fig. 4.21); the presence of pronotal carinae; the absence of propodeal carinae; and antenna with rhinaria and club shape beginning on $\mathrm{F} 4$ in females and $\mathrm{F} 3$ in males. It is similar to $A$. obscurata, but can be differentiated by the proportions of the flagellomere (F1 is subequal to F2, F2 is longer than F3, and F3 is subequal to F4 in A. macrophadna (Fig. 2.25), while $\mathrm{F} 1$ is longer than $\mathrm{F} 2, \mathrm{~F} 2$ is shorter than $\mathrm{F} 3$, and $\mathrm{F} 3$ is shorter than F4 in A. obscurata (Fig. 2.31)), flagellomere shape (F2 and F3 are strongly curved in A. macrophadna males, but no flagellomeres are curved in A. obscurata males), and the size of the radial cell (3.0 times as long as wide in A. macrophadna (Fig. 4.21) but 2.7 times in A. obscurata (Fig. 5.3)).

\section{Material examined}

\section{Lectotype}

GERMANY: [ [] , [lectotype H.H. Evenhuis (orange label)], [Xystus macrophadnus Hartig det. H.H. Evenhuis 1974], [Alloxysta macrophadna (Hartig, 1841) $ᄋ$ M. Ferrer-Suay det. 2011] (ZSM).

\section{Paralectotypes}

GERMANY: 3 q $\bigcirc$ [In collection Hartig as Xystus macrophadnus; Paralectotype Xystus macrophadnus Hartig, 1841 (red label); Alloxysta macrophadna (Hartig, 1841) ㅇ M. Ferrer-Suay det. 2011] (ZSM). 
Additional specimens $(33 \hat{\partial} \widehat{\partial}, 39 q+$ )

CZECH REPUBLIC: 1 ㅇ [Moravia, Zidlochovice, 20 Nov. 84, N.D. Springate] (BMNH); 1 [central Bohemia, 30 Jul. 1991, J. Macek] (CNCI C-278); 1 ð [Moravia, Lanzhot-Ranspurk, 9-12 Aug. 1991, L. Masner, climax hardwood forest, PT] (CNCI C-270); 1 ㅇ [Moravia, Ladnice environs 7-9 Aug. 1991, L. Masner riparian, forest] (CNCI C-272).

FINLAND: 2 $q$ [Helsingfords, 19 Sep. 1933, G.J. Kerrich] (BMNH B.M. 1969-261); 1 [ [Kevo, Res. Station, 69² $45^{\prime}$ N, $27^{\circ} 00^{\prime}$ E, 24 Jun. 1989, H. Goulet, sweep] (CNCI C-309).

FRANCE: $1 \lesssim$ [Haute Savoie, Bossy sur Frangy, 17-30 Aug. 1993, J. Steffen, on wood pile] (CNCI C-289).

GERMANY: 1 ㅇ [Wern, Riedenerwald, 15 Aug. 1978, L. Huggert, sweep in open oak wood] (CNCI C-234); 1 ô, 5 우 [Schwarzwald, Todtmoos environs, 6 Aug. 1984, L. Masner, screen sweeping] (CNCI C-235).

IRELAND: 1 [Co. Donegal: Saggart A.W. Stelfox, 29 Sep. 1935 (green label), Saggar (n. Dv. Aws, 29.9.35) (handwritten, green label), A.W. Stelfox Collection 1966] (USNM).

ITALY: 1 § [Dolomites Seis am Schlern., 1-13 Jun. 1964] (BMNH).

JAPAN: 1 ภ, 2 우 [Hokkaido, Aizan, 800 m, 4 Jul. 1989, sweep, M.J. Sharkey] (CNCI C-186); 1 ㅇ

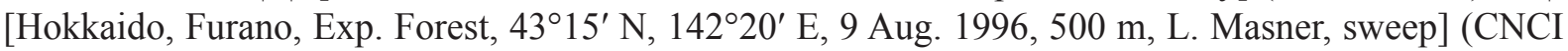

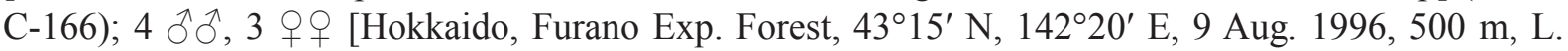
Masner, sweep] (CNCI C-162); 1 q [Hokkaido, Sapporo, Jozankei, 21-28 Sep. 1989, K. Maeto and M. Sharkey] (CNCI C-193); 4 ठิ $\widehat{\partial}$ [Gifu, Mt. Ena-san (ssw), 1200-1900 m, 5 Jun. 1995, K. Yamagishi]

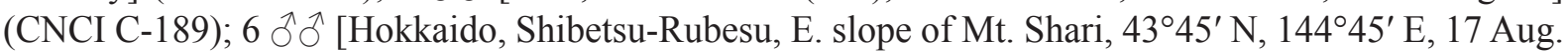
1996, 700 m, L. Masner, sweep] (CNCI C-164); 3 ồ, 1 q [Hokkaido, Furano, Exp. Forest, 43ำ15' N, $142^{\circ} 20^{\prime}$ E, 9 Aug. 1996, 500 m, L. Masner, sweep] (CNCI C-163).

NORWAY: 1 ㅇ [Trondhem, 3 Jul. 1980, F. Joldsa] (BMNH).

POLAND: 1 [Przemysl, 12 Sep. 1984, N.D. Springate] (BMNH).

RUSSIA: 1 ㅇ [Magadanskaya Oblast, Aborigen Field Station, 500 m, 25 Jul.-10 Aug. 1990, D.M. Wood] (CNCI C-298).

SLOVAKIA: 1 q [Košice, 14-17 Sep. 84, N.D. Springate] (BMNH).

SLOVENIA: 1 + [Slov. Vrata Valley, 13 Jun. 1979, P.H. and S.L. Ward] BMNH B.M. 1979-263); 1 ๙ [Rupa, 28 Apr. 1975, J.S.N., Grassland] (BMNH); 1 đ [Slovenia, Slatna, Radovljica, 6 Aug. 1978, L. Huggert, swept on open wooded (deciduous) meadow] (CNCI C-275).

SWEDEN: 1 đ, 8 우 [Sk. Lund, Zoological Mus. grounds, Aug. 1976] (BMNH); 1 , 2 우우 [Sk. Röstanga, 6 Jul. 1938, D.M.S.P. and J.F.P.] (BMNH B.M. 1938-414); 1 ㅇ [Kalmar, Överum, 19 May-29 Jun. 1978, L. Huggert, MT at swampy lake shore] (CNCI C-312); 1 q [Värmland, Ekshärad, 20-29 Jul. 1960, W.R.M. Mason] (CNCI C-313).

SWITZERLAND: 7 ふぇ, 1 ㅇ [Dielsdorf, 650 m, 17 Aug. 1984, L. Masner, sweep] (CNCI C-290).

UNITED KINGDOM: Scotland: 1 \& [Perth and Kinross, Acham, PM, A.W. Stelfox, 15 Jul. 1951, A.W. Stelfox Collection 1966] (USNM). 


\section{Distribution}

Holarctic.

Certain records: Andorra (Ferrer-Suay et al. 2011: 357), England (Müller et al. 1999: 346), Germany (Hartig 1841: 352; Höller et al. 1993: 13), Iran (Ferrer-Suay et al. 2013a: 38), Montenegro (Ferrer-Suay et al. 2013b: 358);

Uncertain records: Austria (Giraud 1860: 130; Hellén 1963: 12), Belgium (Lameere 1907; Crèvecoeur \& Maréchal 1933), Bulgaria and Balkan peninsula (Vasileva-Sumnalieva 1976: 23), England (Cameron 1889: 53; Kieffer 1902a: 10), Finland (Hellén 1963: 12), France (Kieffer 1902a: 10; De Gaulle 1908: 26), Ireland (O’Connor \& Nash 1997), Italy (Hellén 1963: 12), Lappland (Hellén 1963: 12), Norway (Hellén 1966: 393), Poland (Kierych 1979b: 14; Krawczyk et al. 2009: 161), Romania (Barnea et al. 2005: 87), Russia (Hellén 1963: 12), Scotland (Cameron 1883: 368; 1886: 53), Sweden (Thomson 1862: 408), Switzerland (Hellén 1963: 12), The Netherlands (Evenhuis 1974: 165), USA (Tennessee) (Andrews 1978: 59).

New records: Czech Republic and Japan.

\section{Alloxysta marshalliana (Kieffer, 1900)}

Figs 2.26, 4.22

Nephycta marshalliana Kieffer, 1900: 114. Type: deposited in BMNH (examined).

Alloxysta marshalliana - Hellén 1931: 5.

\section{Diagnosis}

Alloxysta marshalliana is mainly characterized as a brachypterous species with a radial cell present. According to these features, A. marshalliana is very similar to A. glebaria, but can be easily differentiated by the shape of the radial cell (completely open in A. marshalliana (Fig. 4.22) but closed in A. glebaria).

\section{Material examined}

\section{Lectotype}

UNITED KINGDOM: England: $\widehat{\delta}$ [Lectotype (round label with blue in the margin)], [Nephycta marshalliana Kieffer = Alloxysta brachyptera ô (nec Hartig) (handwritten, orange label)], [Cameron 96-76 Clober Wa], [Lectotype Nephycta marshalliana K. §̃, det. J. Quinlan, 1977], [B.M. Type Hym 7.173] (BMNH B.M. Type Hym 7.173).

Additional specimens $(3 \widehat{\partial} \widehat{\jmath}, q$ not known)

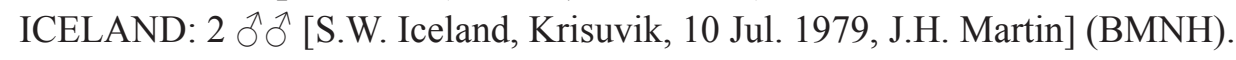

GERMANY: $1 \precsim$ [Schwarzwald, Todtmoos environs, 6 Aug. 1984, L. Masner, screen sweeping] (CNCI C-235).

\section{Distribution}

Europe: England (Kieffer 1900: 114), Romania (Ionescu 1969: 236).

New records: Iceland and Germany. 


\section{Alloxysta melanogaster (Hartig, 1840)}

Figs 2.27, 4.23

Xystus melanogaster Hartig, 1840: 200. Type: deposited in ZSM (examined).

Allotria melanogaster - Giraud 1860: 129.

Allotria (Allotria) melanogaster - Dalla Torre \& Kieffer 1902: 40.

Alletria melanogastra - Lameere 1907: 195.

Charips (Charips) melanogaster - Dalla Torre \& Kieffer 1910: 279.

Alloxysta melanogaster - Hellén 1963: 21.

\section{Diagnosis}

Alloxysta melanogaster is mainly characterized by a partially open radial cell that is 2.3 times as long as wide (Fig. 4.23); the presence of pronotal and propodeal carinae; female antenna with rhinaria beginning on F3, F1 subequal to pedicel, F1 longer than F2, F2 subequal to F3, and F4 longer than F3 (Fig. 2.27); male antenna with rhinaria beginning on F2, F1 longer than pedicel and F2, and F2-F4 subequal in length. It is similar to A. longipennis, but can be differentiated by the proportions of the flagellomere in females ( $\mathrm{F} 1$ is subequal to pedicel in A. melanogaster (Fig. 2.27), while F1 is longer than pedicel and F2 and F2 is subequal to F3 in A. longipennis (Fig. 2.24)) and the size of the radial cell (2.3 times as long as wide in A. melanogaster (Fig. 4.23) but 2.6 times in A. longipennnis (Fig. 4.20)).

\section{Material examined}

\section{Lectotype}

GERMANY: $\widehat{o}[\widehat{\jmath}]$, [lectotype H.H. Evenhuis (orange label)], [Xystus melanogaster Hartig det. H.H. Evenhuis 1980], [Alloxysta melanogaster (Hartig, 1840) §̊ M. Ferrer-Suay det. 2011] (ZSM).

\section{Paralectotype}

GERMANY: 1 o [314], [In collection Hartig as Xystus melanogaster], [Paralectotype Xystus melanogaster Hartig, $1840 \widehat{\partial}$ (red label)], [Alloxysta melanogaster (Hartig, 1840) $\overbrace{}^{\lambda} \mathrm{M}$. Ferrer-Suay det. 2011] (ZSM).

\section{Additional specimens ( 1 )}

JAPAN: 1 q [Aichi, Midori -Ku, Nagoya city, 2 Jun. 1984, R. Baczyuski] (CNCI C-180).

\section{Distribution}

Palaearctic and Oriental.

Certain records: Germany (Hartig 1840: 200), Iran (Ferrer-Suay et al. 2013a: 38), Italy (Ferrer-Suay et al. 2014b), Japan, Thailand and Taiwan (Ferrer-Suay et al. 2013i).

Uncertain records: Austria (Giraud 1860: 129), Belgium (Lameere 1907), Finland (Hellén 1963: 21), France (De Gaulle 1908: 26; Dalla Torre \& Kieffer 1910: 279), Romania (Ionescu 1969: 252), Scotland (Cameron 1886: 86).

Alloxysta mullensis (Cameron, 1883)

Figs 2.28, 4.24

Allotria mullensis Cameron, 1883: 366. Type: deposited in BMNH (examined).

Allotria (Allotria) mullensis - Dalla Torre \& Kieffer 1902: 40. 
Charips (Charips) mullensis - Dalla Torre \& Kieffer 1910: 284.

Alloxysta mullensis - Quinlan 1974: 8.

\section{Diagnosis}

Alloxysta mullensis is mainly characterized by a closed radial cell that is 2.2 times as long as wide (Fig. 4.24); the absence of pronotal carinae; the presence of propodeal carinae that form a plate; and male and female antenna with rhinaria beginning on F4, F1 longer than F2, F2 subequal to F3, and F3 shorter than F4 (Fig. 2.28). It is similar to A. fracticornis, but can be differentiated by the proportion of the F1-pedicel ratio (F1 is subequal to pedicel in A. mullensis (Fig. 2.28), but longer than pedicel in A. fracticornis (Fig. 2.17)), flagellomere proportions (F1 is longer than F2 and F2 is subequal to F3 in A. mullensis females (Fig. 2.28), while F1-F3 are subequal in length in A. fracticornis females (Fig. 2.17)), and flagellomere shape (no flagellomeres are curved in A. mullensis males, but F3 is curved in A. fracticornis males).

\section{Material examined}

\section{Lectotype}

UNITED KINGDOM: Scotland: $q$ [Holotype (round label with red in the margin)], [mullensis (handwritten)], [mullensis Cam (handwritten)], [Cameron. 96 - 76., Mull, on the underside of this label is the name mullensis], [Holotype $q$ of Allotria mullensis Cameron. det. J. Quinlan, 1973 (white label)], [B.M. Type HYM. 7.125 (white label)], [Lectotype Allotria mullensis $q$ Cameron, 1883 design. FerrerSuay and J.P-V 2011 (red label)], [Alloxysta mullensis (Cameron, 1883) 中, Ferrer-Suay and J.P-V det. (white label)] (BMNH B.M. Type HYM. 7.125).

Additional specimens $(5 \hat{\jmath} \widehat{\partial}, 24$ 우)

DENMARK: 1 [S. Zealand, Feddet near Praesto, 27 Aug. 1994, grassy marsh area] (CNCI C-241); $1 \lesssim$ [W. Zealand, Gyrstinge, 20 km S of Holbaek, 28 Aug. 1994] (CNCI C-243).

FRANCE: 1 [Fonscolombe (2), 7 Jun. 1982, M.W.R. de V. Graham coll.] (BMNH B.M. 1995-177) (handwritten).

GERMANY: 1 \& [Hesse, Gelnhausen, Sep. 1967, MT, A.W. Steffan] (CNCI C-228).

IRAQ: $1 \lesssim$ [Ex Aphis craccivora on Horse Bea, Coll. 3 Apr. 1964 (handwritten), Trioxys angelicae Hal., Associated with Lysiphlebus fabarum Marsh., Pachyneuron aphidis Bouché (handwritten), Iraq Baghdad, A. Al-Azawi (handwritten), Charips ㅇ? sp. det. J. Quinlan, 1964] (BMNH B.M. 1964-516).

JAPAN: 1 [ [Kyushu, Fukuoka, Mt. Hiko, 700 m, 9-10 May 1989, M.J. Sharkey Sweep] (CNCI C-138); 1 o [Mt. Hayachine, 5-11 Jul. 89, Makihara and M. Sharkey, 400 m, MT] (CNCI C-173); 1 q [Fukuoka, Mt. Hiko, 10-21 Jul. 1989, Takeno and M. Sharkey] (CNCI C-154); 3 q 9 [Hokkaido, Sapporo, Jozankei, 350 m, Aug. 1989, K. Maeto and M. Sharkey, MT] (CNCI C-203); 3 우 [Hokkaido, Sapporo, Jozankei, 350 m, 12-21 Sep. 1989, K. Maeto and M. Sharkey, MT] (CNCI C-205); 3 q $q$ [Hokkaido, Sapporo, Jozankei, 350 m, 21-29 Aug. 1989, K. Maeto and M. Sharkey, MT] (CNCI C-202); 1 ภ̊, 3 q $q$ [Hokkaido, Sapporo, Jozankei, 350 m, 10-21 Aug. 1989, K. Maeto and M. Sharkey, MT] (CNCI C-206).

RUSSIA: 2 ふぇ, 4 우 [Primoskiy Kray Ussyriysk District, Gomotayozhnoye, 11-15 Aug. 2003, Malaise trap, M.V. Michailovskaya] (USNM).

SPAIN: 1 q [Madrid, Cercedilla, 22 Oct. 1978, J.S. Noyes] (BMNH B.M. 1978-488).

SLOVENIA: 1 q [C-276, Yugoslavia: Slovenia, Radovljica, 3 Aug. 1978, L. Huggert, swept on dry meadow] (CNCI). 


\section{Distribution}

Paleaearctic and Neotropical.

Certain records: France (Ferrer-Suay et al. 2015a), Italy (Ferrer-Suay et al. 2014b), Mexico (FerrerSuay et al. 2013m: 37), Spain (Ferrer-Suay et al. 2013g), Montenegro (Ferrer-Suay et al. 2013b: 358), Scotland (Cameron 1883: 366; 1886: 86), Slovenia (Ferrer-Suay et al. 2013b: 358).

Uncertain records: Iran (Lotfalizadeh 2002a: 36), Russia (Bokina 1997: 435);

New records: Denmark, France, Germany, Iraq, Japan and Spain.

\section{Alloxysta nigricans Hellén, 1963}

Figs 2.29, 5.1

Alloxysta nigricans Hellén, 1963: 16. Type: deposited in MZH (examined).

\section{Diagnosis}

Alloxysta nigricans is mainly characterized by a closed radial cell that is 2.7 times as long as wide, the presence of pronotal carinae and a propodeal plate, and antenna with rhinaria and club shape beginning on F4 in both males and females and F1 longer than pedicel (Fig. 2.29). There is no other known species of Alloxysta similar to A. nigricans.

\section{Material examined}

\section{Holotype}

FINLAND: + [Taipalsaari], [Fennia], [Hellén], [2920], [not understandable (handwritten)], [nigricans m (handwritten)], [Holotypus (red label)], [Mus. Zool. Helsinki, Loan No. HY 4122 (yellow label)], [Mus. Zool. Helsinki, Loan No HY 2012 - 1831 (yellow label)], [Alloxysta nigricans Hellén, 1963 M. Ferrer-Suay det. 2012] (ZMH).

\section{Paratype}

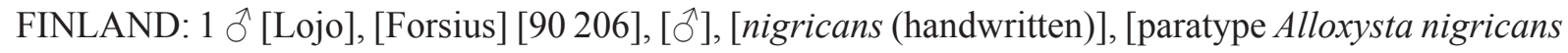
Hellén (handwritten) (red label)], [Mus. Zool. Helsinki, Loan No. HY 2012 - 1833 (yellow label)], [Alloxysta nigricans Hellén, 1963 ð M. Ferrer-Suay det. 2012] (ZMH).

\section{Distribution}

Europe.

Certain records: Finland (Hellén 1963: 16).

\section{Alloxysta nigrita (Thomson, 1862)}

Figs 2.30, 5.2

Allotria nigrita Thomson, 1862: 409. Type: deposited in MZLU (examined).

Dilyta nigrita - Kieffer 1900: 114.

Alloxysta (Alloxysta) nigrita - Dalla Torre \& Kieffer 1902: 39.

Alloxysta nigrita-Andrews 1978: 87. 


\section{Diagnosis}

Alloxysta nigrita is mainly characterized by a completely open radial cell that is 2.9 times as long as wide in both males and females (Fig. 5.2); the presence of pronotal carinae; the absence of propodeal carinae; antenna with rhinaria beginning on F4 in both males and females; female antenna with F1 longer than pedicel and F2, F2 shorter than F3, and F3 longer than F4 (Fig. 2.30); male antenna with F1 longer than pedicel and subequal to F2, F2 longer than or subequal to F3, and F3 shorter than F4. It is similar to A. brachycera, but can be differentiated by the proportion of F2-F3 (F2 is shorter than F3 in A. nigrita (Fig. 2.30), but longer than F3 in A. brachycera (Fig. 2.7)) and the size of the radial cell (2.9 times as long as wide in A. nigrita (Fig. 5.2), but 2.7 times in A. brachycera (Fig. 4.6)).

\section{Material examined}

\section{Lectotype}

SWEDEN: $q$ [L-d], [Lectotype Allotria nigrita Thomson, 1862 q desig. M. Ferrer-Suay 2011 (red label)], [Alloxysta nigrita (Thomson, 1862) $\odot$ M. Ferrer-Suay det. 2011], [ZML. 2011065 (green label)] (MZLU).

\section{Paralectotype}

SWEDEN: $q$ [Lund], [Paralectotype Allotria nigrita Thomson, 1862 q (red label)], [Alloxysta nigrita (Thomson, 1862) $q$ M. Ferrer-Suay det. 2011] (MZLU).

Additional specimens $(1+$ )

UNITED KINGDOM: England: 1 \& [Surrey Chobham Common, 26 Aug. 1982, E.E. Grissell, sweeping oak] (BMNH).

\section{Distribution}

Palaearctic.

Certain records: Madeira (Ferrer-Suay et al. 2012g: 11), Sweden (Thomson 1862: 409).

Uncertain records: Bulgaria and Balkan peninsula (Vasileva-Sumnalieva 1976: 23), France (Kieffer 1902b: 44), Hungary (Fülöp et al. 2010: 55), Israel (Argaman 1988: 114), Romania (Barnea et al. 2005: 87).

New record: England.

\section{Alloxysta obscurata (Hartig, 1840)}

Figs 2.31, 5.3

Xystus obscuratus Hartig, 1840: 200. Type: deposited in ZSM (examined).

Allotria obscuratus - Taschenberg 1866: 130.

Allotria obscurata - Dalla Torre 1893: 34.

Dilyta obscurata - Kieffer 1900: 114.

Alloxysta (Alloxysta) obscurata - Dalla Torre \& Kieffer 1902: 39.

Alloxysta obscurata - Andrews 1978: 87.

\section{Diagnosis}

Alloxysta obscurata is mainly characterized by a partially open radial cell that is 2.7 times as long as wide (Fig. 5.3); the presence of pronotal carinae; the absence of propodeal carinae; female antenna with 
rhinaria beginning on F3, F1 longer than pedicel and F2, F2 subequal to F3, and F3 shorter than F4 (Fig. 2.31); male antenna with rhinaria beginning on F4, F2 slightly curved, F1 longer than pedicel and $\mathrm{F} 2$, F2 longer than F3, and F3 longer than F4. It is similar to A. macrophadna, but can be differentiated by the proportions of the flagellomere (F1 is longer than F2, F2 is shorter than F3, and F3 is shorter than $\mathrm{F} 4$ in A. obscurata (Fig. 2.31), while $\mathrm{F} 1$ is subequal to $\mathrm{F} 2, \mathrm{~F} 2$ is longer than $\mathrm{F} 3$, and $\mathrm{F} 3$ is subequal to $\mathrm{F} 4$ in A. macrophadna (Fig. 2.25)), the shape of the flagellomere (no flagellomeres are curved in males of A. obscurata, but $\mathrm{F} 2$ and $\mathrm{F} 3$ are strongly curved in males of $A$. macrophadna), and the size of the radial cell (2.7 times as long as wide in A. obscurata (Fig. 5.3), but 3.0 times in A. macrophadna (Fig. 4.21)).

\section{Material examined}

\section{Lectotype}

GERMANY: + , [second from top, lectotype H.H. Evenhuis (orange label)], [Xystus obscuratus Hartig det. H.H. Evenhuis 1980], [Alloxysta obscurata (Hartig, 1840) $ᄋ$ M. Ferrer-Suay det. 2011] (ZSM).

\section{Paralectotype}

GERMANY: + , [Xystus obscuratus Hartig det. H.H. Evenhuis 1980], [first from top, Paralectotype

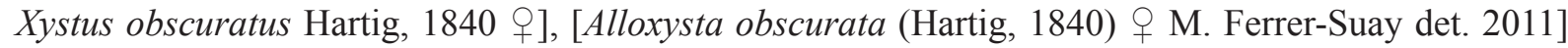
(ZSM).

Additional specimens $(9 \hat{\partial} \hat{\partial}, 52$ q $q)$

AUSTRIA: 1 \& [Semmeringgebiet, Reichenan dist., 25 May-6 Jun. 1957, G.E.J. Nixon] (BMNH).

CROATIA: 1 [swept from vegetation by lake, Croatia, Plitvice, 4-10 Aug., Jugoslavia: 1955, R.L. Coe.] (BMNH B.M. 1955-460).

CZECH REPUBLIC: 3 q 9 [Moravia, Dyje River, near Znojmo, 12 Aug. 91, L. Masner] (CNCI C-263); 1 \& [Moravia, Lanzhot-Ranspurk, 7-9 Aug. 1991, L. Masner, sweep, climax flood forest] (CNCI C-268); 2 우 [Moravia, 16 km N Blansko, 8 Aug. 1991, L. Masner, sweep. Tilia-Acer forest] (CNCI C-271); 1 ô [Moravia, Lanzhot-Ranspurk, 9-12 Aug. 1991, L. Masner, climax hardwood forest, PT] (CNCI C-270); 1 q [Moravia, Ladnice environs 7-9 Aug. 1991, L. Masner riparian, forest] (CNCI C-272).

GERMANY: 3 우 [Schwarzwald, Todtmoos environs, 6 Aug. 1984, L. Masner, screen sweeping] (CNCI C-235).

JAPAN: 1 § [Fukuoka, Mt. Hiko, 700 m, 9-10 May 1989, sweep, M.J. Sharkey] (CNCI C-183); 2 우 [Hokkaido, Sapporo, Jozankei, 350 m, 20-31 Jul. 1989, K. Maeto and M. Sharkey] (CNCI C-140); 4 우 [Hokkaido, Sapporo, Jozankei, 350 m, 20-31 Jul. 1989, K. Maeto and M. Sharkey] (CNCI C-151); 2 우오 [Hokkaido, Sapporo, Jozankei, 350 m, Aug. 1989, K. Maeto and M. Sharkey, MT] (CNCI C-203);

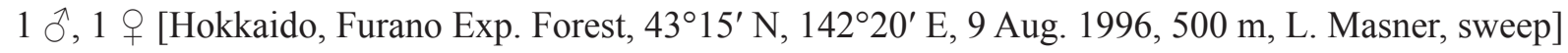

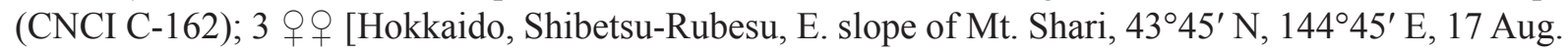
1996, 700 m, L. Masner, sweep] (CNCI C-164); 1 \& [Hokkaido, Sapporo, Jozankei, 350 m, 20-31 Jul. 1989, MT, K. Maeto and M. Sharkey] (CNCI C-204); 3 우 [Hokkaido, Sapporo, Jozankei, 350 m, 1021 Aug. 1989, K. Maeto and M. Sharkey, MT] (CNCI C-206); 1 q [Hokkaido, Nukabira, 600 m, 5 Jul. 1989, sweep, M.J. Sharkey] (CNCI C-215); 1 q [Hokkaido, Sapporo, Jozankei, 350 m, 29 Aug.-12 Sep. 1989, K. Maeto and M. Sharkey, MT] (CNCI C-207).

POLAND: 1 [ [Przemysl, 12 Sep. 1984, N.D. Springate: 1 †] (BMNH).

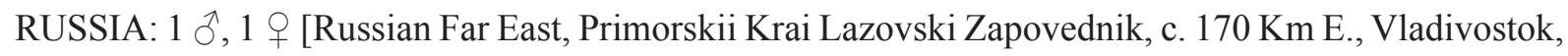
Lazo, 4330'33" N, 13406'59" E, 1375 m, 2 Jun.-3 Jul. 2001, Mountain top, malaise trap 458, M. Quest 
coll., Alloxysta sp1., det. M. Forshage 2012] (BMNH B.M.2009-59); 1 q [Magadanskaya Oblast, Aborigen Field Station, 500 m, 25 Jul.-10 Aug. 1990, D.M. Wood] (CNCI C-298).

SLOVAKIA: 1 ô, 3 우 [Čachtice environs, 2 Aug. 1991, L. Masner, sweep] (CNCI C-267); Koš̀ice, 14-17 Sep. 1984, N.D. Springate] (BMNH).

SWEDEN: 1 [Sk. Röstanga, 6 Jul. 1938, D.M.S.P. and J.F.P.] (BMNH B.M. 1938-414); 1 q [Sk. Kivik, 19 Jul. 1938, D.M.S.P and J.F.P.] (BMNH B.M. 1938-414).

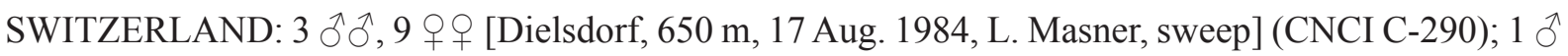
[Jura, Delémont, CABI Lab., 47²2'42" N, 7²0'30" E, 7 Jun. 1999, H. Goulet, spruce/beech] (CNCI C-287); 1 ㅇ [Solothurn, Weissenstein, 1225 m, 47 $7^{\circ} 15^{\prime} 10^{\prime \prime}$ N, 7³0'00" E, 17 Jun. 1999, Goulet/White, lush meadow] (CNCI C-285); 3 우 [Turgau, Unterwasser environs, 1440 m, 4 Aug. 1984, L. Masner, sweeping] (CNCI C-293).

\section{Distribution}

Holartic.

Certain records: Andorra (Ferrer-Suay et al. 2011: 358), Colombia (Ferrer-Suay et al. 2012f: 325), Germany (Hartig 1840: 200; Hübner et al. 2002: 507), Japan (Ferrer-Suay et al. 2013i).

Uncertain records: Canada (Vancouver Island) (Andrews 1978: 70), Canada (British Columbia) (Andrews 1978: 56), Canada (Quebec) (Bouchard et al. 1982: 184), France (Kieffer 1902b: 597; De Gaulle 1908: 26), Hungary (Fülöp et al. 2010: 55), Iceland (Andrews 1978: 87), Poland (Kierych 1979b: 15), Romania (Ionescu 1969: 267), Scotland (Cameron 1886: 88), USA (Alaska) (Andrews 1978: 70), USA (Colorado) (Baker 1896: 132,134).

New records: Austria, Czech Republic, Croatia, Japan, Poland, Russia, Sweden and Switzerland.

Alloxysta palearctica Ferrer-Suay \& Pujade-Villar sp. nov. urn:Isid:zoobank.org:act:E3599771-EFEE-468C-83BC-9B49CFCA5DBA

Fig. 11

\section{Diagnosis}

Alloxysta palearctica sp. nov. is mainly characterized by its completely open radial cell, pronotal carinae present, propodeal plate present, placodeal sensilla and club shape begin on F4, F1 equal to pedicel, F1 and $\mathrm{F} 2$ very bumped. The combination of these features easily distinguishes $A$. palearctica sp. nov. from other species of Alloxysta.

\section{Etymology}

This new species is named after the biogeographic region where it was collected.

\section{Material examined}

Holotype

JAPAN: $\lesssim$ [Hokkaido, Nukabira, 600 m, 5 Jul. 1989, sweep, M.J. Sharkey] (CNCI C-215).

\section{Description}

Length. Female: unknown. Male: $0.9 \mathrm{~mm}$. 

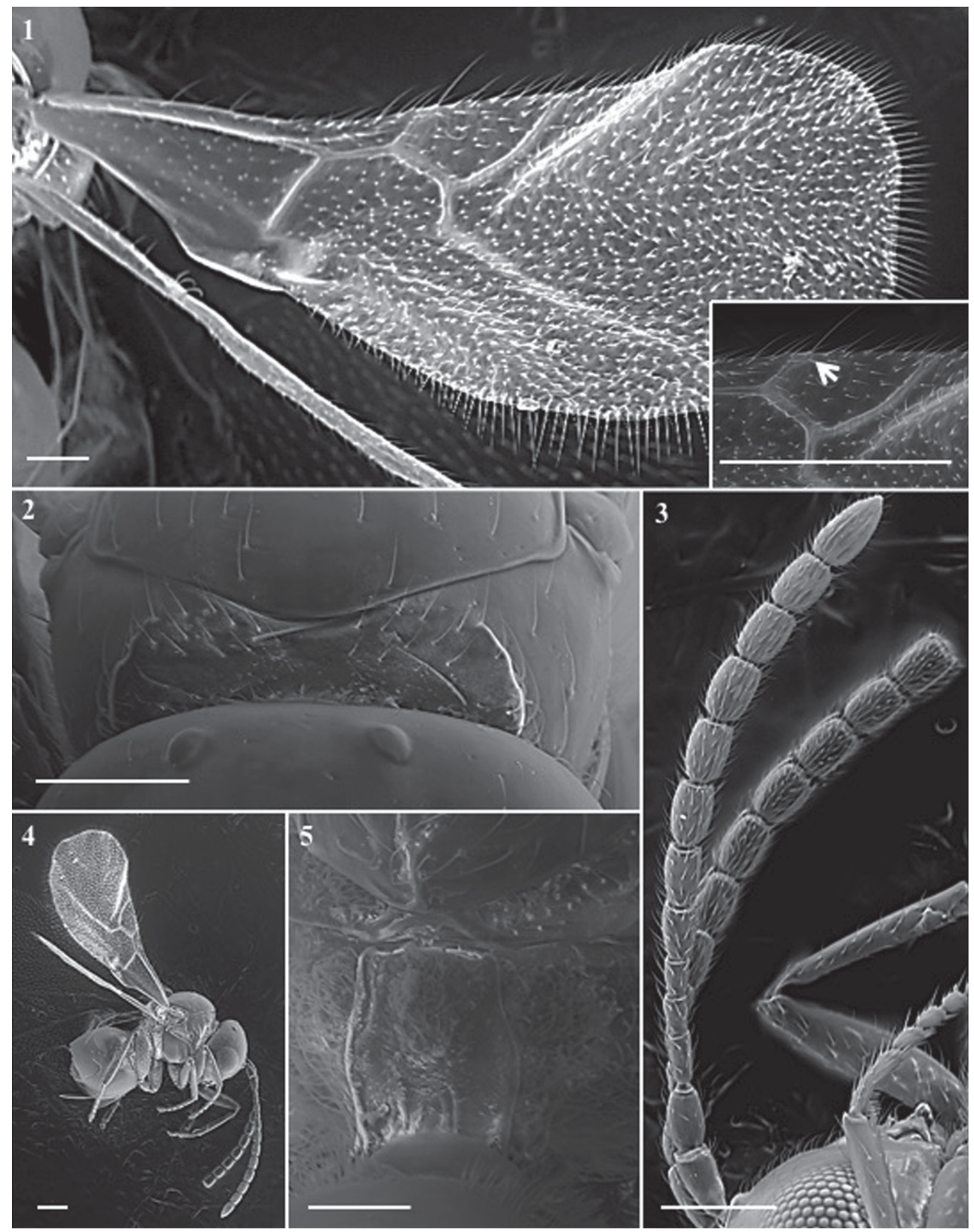

Fig. 11. Alloxysta pascuali Ferrer-Suay sp. nov. 1. Fore wing. 2. Pronotum. 3. Antenna. 4. Body. 5. Propodeum. Scale bars: $50 \mu \mathrm{m}$. 
Coloration. Head, mesosoma and metasoma yellowish brown. Antenna yellowish slightly darkening towards the end. Legs yellow and veins yellowish brown.

HEAD. Transversely ovoid, smooth and shiny, slightly wider than high in front view. Setae below and between toruli, few scattered setae above toruli. Scattered setae on vertex and many setae on face. Transfacial line 0.8 times height of compound eye. Malar space 0.2 times height of compound eye.

ANTENNA. 14-segmented, filiform. All antennomeres covered with sparse setae. F1-F3 smooth and thinner than remaining flagellomeres, F4-F11 with placodeal sensilla and club shaped. Antennal formula: 4.5 (2.7); 4.5 (2.0); 4.4 (2.1); 4.0 (2.0); 4.8 (2.5); F4-F11 subequal in length, width and shape (Fig. 11.3).

Mesosoma. Pronotum covered by few sparse setae, carinae present (Fig. 11.5). Mesoscutum smooth and shiny, round in dorsal view with few scattered setae. Scutellum smooth and shiny with scattered setae, more abundant on apex of scutellum. Propodeum covered by abundant pubescence, with two carinae forming a protruding plate, this plate has a few setae on anterior third (Fig. 11.7).

ForE WING. Longer than body, 1.4 times as long as mesosoma and metasoma together. Covered with dense pubescence; marginal setae present and very long (Fig. 11.1). Completely open radial cell, 2.3 times as long as wide. R1 short and curved; Rs long and slightly curved (Fig. 11.4).

Metasoma. Anterior part with an incomplete ring of setae, glabrous at center, wider laterally. Metasoma smooth and shiny, T3 and T4 clearly distinguished.

\section{Distribution}

Eastern Palaearctic: Japan.

Alloxysta pallidicornis (Curtis, 1838)

Figs 2.32, 5.4

Cynips pallidicornis Curtis, 1838: 688 (April 1). Type: deposited in MVMA (examined).

Alloxysta pallidicornis - Quinlan \& Fergusson 1981: 254.

\section{Diagnosis}

Alloxysta pallidicornis is easily differentiated from other Alloxysta species by the following combination of features: a completely open radial cell (Fig. 5.4); the presence of pronotal carinae; propodeal carinae that are well-defined, are separated by setae in the anterior half, and form a plate in the posterior half; and antenna with rhinaria beginning on F2 and F1 and a very large length-to-width ratio (Fig. 2.32).

\section{Material examined}

\section{Lectotype}

UNITED KINGDOM: England: $q$ [Holotype (round label with red margins)], [Holotype of Cynips pallidicornis Curt. det. Fergusson and Quinlan 1980], [Alloxysta forticornis (Gir.) $q$ det J. Quinlan, 1980], [MUS. VIC. ENTO 2011-IIL (green label)], [Lectotype Cynips pallidicornis Curtis $\bigcirc$ design. M. Ferrer-Suay 2013], [Alloxysta pallidicornis (Curtis, 1838) M. Ferrer-Suay det. 2011] (MVMA).

\section{Distribution}

Eastern Palaearctic, Nearctic and Nepal.

Certain records: England (Curtis 1838: 688; Fergusson 1986: 19), Nepal (Ferrer-Suay et al. 2013i), USA (Evenhuis \& Kiriak 1985: 19). 
Uncertain records: Austria (Giraud 1860: 130), Finland (Hellén 1963: 11), France (Andrews 1978: 83), Germany (Zetterstedt 1838: 410; Taschenberg 1866: 129; Hübner et al. 2002: 507), Norway (Zetterstedt 1838: 410; Hellén 1963: 11), Spain (Torras-Casals 1996: 196), Sweden (Thomson 1862: 408), Canada and USA (Andrews 1978: 54).

Alloxysta pascuali Ferrer-Suay sp. nov. urn:1sid:zoobank.org:act:C6E39FE2-320A-4425-8CC7-5D73706CAF38

Fig. 12

\section{Diagnosis}

Alloxysta pascuali sp. nov. is mainly characterized by a completely open radial cell; the presence of pronotal carinae and a propodeal plate; antenna with placodeal sensilla, club shape beginning on $\mathrm{F} 4$, and F1 shorter than pedicel. The combination of these features readily distinguishes $A$. pascuali sp. nov. from its congeners.

\section{Etymology}

This new species is dedicated to Pascual Mas Palomares, brother-in-law of the first author, who wants to thank him for his constant support.

\section{Material examined}

Holotype

JAPAN: ㅇ [Kyushu, 700 m, Fukuoka, MT. Hiko, 10-21 Jul. 1989, MT, K. Takeno and M. Sharkey] (CNCI C-171).

Paratypes $(2+\circ)$

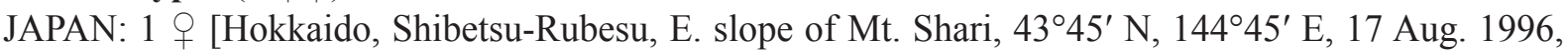
700 m, L. Masner, sweep] (UB).

GERMANY: 1 \& [Schwarzwald, Todtmoos environs, 6 Aug. 1984, L. Masner, screen sweeping] (CNCI C-235).

\section{Description}

LeNGTH. Female: 0.82-0.99 mm. Male: unknown.

Coloration. Head, mesosoma and metasoma yellowish brown. Scape, pedicel, F1-F3 dark yellow, F4F11 yellowish brown. Legs yellow and veins yellowish brown.

HEAD. Transversely ovoid, smooth and shiny, slightly wider than high in front view. Setae below and between toruli, few scattered setae above toruli. Scattered setae on vertex and many setae on face. Transfacial line 0.8 times height of compound eye. Malar space 0.5 times height of compound eye.

ANTENNA. 13-segmented, filiform. All antennomeres covered with sparse setae. F1-F3 smooth and thinner than remaining flagellomeres, F4-F11 with placodeal sensilla and club shaped. Antennal formula: 3.5 (2.0); 2.5 (1.3); 2.0 (1.3); 2.0 (1.3); 2.8 (2.0); F4-F11 subequal in length, width and shape (Fig. 12.3).

Mesosoma. Pronotum covered by sparse setae, fewer in distolateral corners and abundant on anterior margins, with two long and curved carinae present (Fig. 12.2). Mesoscutum smooth and shiny, round in dorsal view with few scattered setae. Scutellum smooth and shiny with scattered setae, more abundant on apex of scutellum. Propodeum covered by abundant pubescence, with two carinae forming a wide and protruding plate, this plate has a few setae on anterior half (Fig. 12.5). 

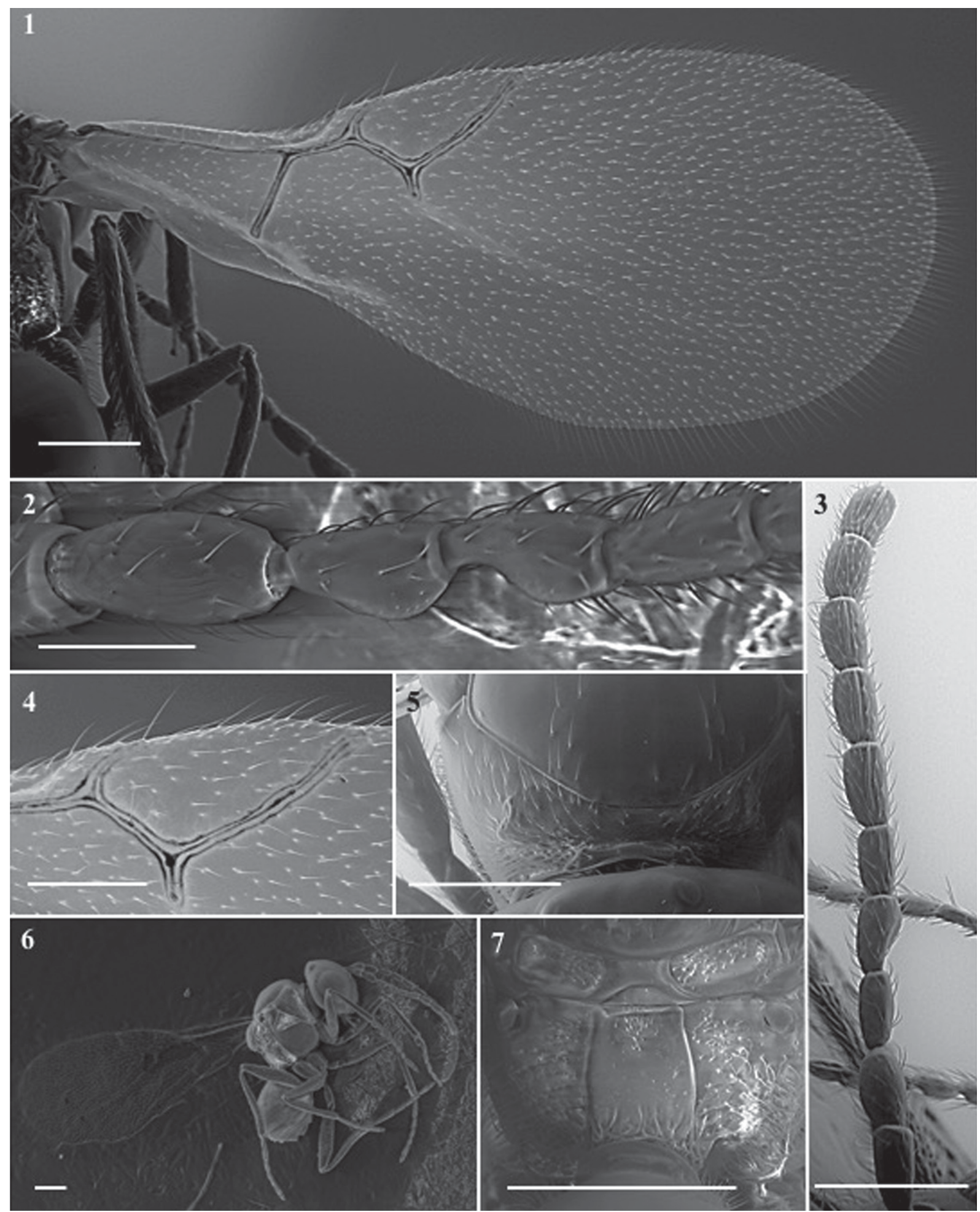

Fig. 12. Alloxysta palearctica Ferrer-Suay \& Pujade-Villar sp. nov. 1. Fore wing. 2. Detail of antenna. 3. Antenna. 4. Radial cell. 5. Pronotum. 6. Body. 7. Propodeum. Scale bars: $50 \mu \mathrm{m}$. 
FORE WING. Longer than body, 1.3 times as long as mesosoma and metasoma together. Covered with dense pubescence; marginal setae present and very long (Fig. 12.1). Completely open radial cell, 2.0 times as long as wide. R1 short and almost straight; Rs long and slightly curved (Fig. 12.1).

Metasoma. Anterior part with an incomplete ring of setae, glabrous at center, wider laterally. Metasoma smooth and shiny, T3 and T4 clearly distinguished.

\section{Distribution}

Eastern Palaearctic: Germany, Japan.

Alloxysta patens Hellén, 1963

Figs 2.33, 5.5

Alloxysta patens Hellén, 1963: 15. Type: deposited in MZH (examined).

\section{Diagnosis}

Alloxysta patens is mainly characterized by a partially open radial cell that is 2.8 times as long as wide; the absence of pronotal and propodeal carinae; and antenna with rhinaria and club shape beginning on F4, F1 subequal to pedicel, F1 longer than F2, F2 subequal to F3, and F3 shorter than F4 (Fig. 2.33). No other known species of Alloxysta is comparatively similar to this one.

\section{Material examined}

\section{Lectotype}

FINLAND: + [Holotype (round label with red margins)], [Holotype of Cynips pallidicornis Curt. det Fergusson and Quinlan 1980], [Alloxysta forticornis (Gir.) ㅇ det J. Quinlan, 1980], [MUS. VIC. ENTO 2011-IIL (green label)], [Lectotype Cynips pallidicornis Curtis + design. M. Ferrer-Suay 2013], [Alloxysta pallidicornis (Curtis, 1838) + M. Ferrer-Suay det. 2011] (MZH).

\section{Distribution}

Europe.

Certain records: Finland (Hellén 1963: 15).

\section{Alloxysta pedestris (Curtis, 1838)}

Fig. 3.1

Cynips pedestris Curtis, 1838: 688. Type: deposited in MVMA (examined).

Allotria pedestris - Cameron 1886: 88.

Nephycta pedestris - Kieffer 1900: 114.

Alloxysta pedestris - Hellén 1963: 19.

\section{Diagnosis}

Alloxysta pedestris is mainly characterized as a brachypterous species without a visible radial cell, without pronotal or propodeal carinae, and with antenna with F1 longer than pedicel and F2, and F2 subequal to F3 (Fig. 3.1). It is similar to A. apteroidea, but can be differentiated by the length of the fore wing (reaching the anterior end of the metasoma in $A$. pedestris, very short, practically absent, in A. apteroidea) and the F1-pedicel proportion (F1 is longer than pedicel in A. pedestris (Fig. 3.1), but shorter than pedicel in A. apteroidea (Fig. 2.3)). 


\section{Material examined}

\section{Lectotype}

UNITED KINGDOM: England: 0 [Lectotype (round label with blue in the margin)], [Holotype of Cynips pedestris Curt. det. Fergusson and Quinlan 1980], [ENT-936; Alloxysta pedestris (Curtis, 1838)

$\hat{\jmath}$ M. Ferrer-Suay det. 2011] (MVMA).

\section{Paralectotype}

UNITED KINGDOM: England: 1 [Paralectotype (round label with blue in the margin)], Type of Cynips pedestris Curt., G.J. Kerrich det. 1948, = Pezophycta p. + ], [MUS. VIC. ENTO 2011-IIL (green label)], [Alloxysta pedestris (Curtis, 1838) ㅇ M. Ferrer-Suay det. 2011] (MVMA).

\section{Additional specimens}

NORWAY: $1 \lesssim$ [Kyrkjtangen Nordåsv, Hoy: Bergen, 1980-05-19, A. Fjeldså leg] (BMNH).

\section{Distribution}

Europe.

Certain records: England (Curtis 1838: 688).

Uncertain records: Austria (Hellén 1963: 19), Denmark (Hellén 1963: 19), Finland (Hellén 1963: 19), Germany (Hellén 1963: 19), Ireland (O’Connor \& Nash 1997; O’Connor 2002: 122), Madeira (Borges et al. 2008), Norway (Hellén 1966: 393), Romania (Ionescu 1969: 237), Scotland (Cameron 1886: 88), Sweden (Dalla Torre \& Kieffer 1910: 292).

\section{Alloxysta piceomaculata (Cameron, 1883)}

Figs 3.2, 5.6

Allotria piceomaculata Cameron, 1883: 367. Type: deposited in BMNH (examined).

Dilyta piceomaculata - Kieffer 1900: 114.

Alloxysta (Alloxysta) piceomaculata - Dalla Torre \& Kieffer 1902: 39.

Alloxysta piceomaculata - Hellén 1963: 13.

\section{Diagnosis}

Alloxysta piceomaculata is mainly characterized by an open radial cell that is 2.8 times as long as wide; the presence of pronotal carinae; the absence of propodeal carinae; and antenna with rhinaria and club shape beginning on F4, F1 longer than pedicel and F2, F2 shorter than F3, and F3 subequal to F4. It is similar to A. nigrita, but can be differentiated by the proportion of the F3-F4 (F3 is subequal to F4 in A. piceomaculata (Fig. 3.2), while F3 is longer than F4 in A. nigrita (Fig. 2.30)) and the shape of the radial cell (R1 and Rs do not reach the margin of the fore wing in A. piceomaculata (Fig. 5.6), but do reach it in A. nigrita (Fig. 5.2)).

\section{Material examined}

\section{Holotype}

UNITED KINGDOM: Scotland: $q$ [Holotype (round label with red margin)], [Cameron 96-76. Dumfries], [Dumfries, piceomaculata (handwritten)], [piceomaculata Cam (handwritten)], [Holotype A Allotria piceomaculata C. det. J. Quinlan, 1973 (handwritten)], [B.M. TYPE HYM. 7.127], [Alloxysta piceomaculata (Cameron, 1883) det. M. Ferrer-Suay 2012] (BMNH B.M. Type Hym. 7.127). 


\section{Distribution}

Europe.

Certain records: Scotland (Cameron 1883: 367).

\section{Alloxysta pilipennis (Hartig, 1840)}

Figs 3.3, 5.7

Xystus pilipennis Hartig, 1840: 199. Type: deposited in ZSM (examined).

Allotria pilipennis - Thomson 1862: 406.

Allotria (Allotria) pilipennis - Dalla Torre \& Kieffer 1902: 40.

Charips (Charips) pilipennis - Dalla Torre \& Kieffer 1910: 283.

Alloxysta pilipennis - Hellén 1963: 19.

\section{Diagnosis}

Alloxysta pilipennis is mainly characterized by a closed radial cell that is 2.5 times as long as wide (Fig. 5.7); the presence of pronotal and propodeal carinae; and female antenna with rhinaria beginning on F3, F1 longer than pedicel and F2, and F2-F4 subequal in length (Fig. 3.3). It is similar to A. pusilla, but can be differentiated by the proportions of the flagellomere in females (F2 is subequal to F3 in A. pilipennis females (Fig. 3.3), but shorter than F3 in A. pusilla females (Fig. 3.7)), flagellomere proportions and shape in males (F1-F3 are not subequal and not curved in A. pilipennis males, but F1-F3 are subequal in length and slightly curved in A. pusilla males), and the size of the radial cell (2.5 times as long as wide in A. pilipennis females (Fig. 5.7), but 2.7 times in A. pusilla females (Fig. 5.11)).

\section{Material examined}

\section{Lectotype}

GERMANY: + [1709 (blue label)], [o], [lectotype H.H. Evenhuis (orange label)], [Xystus pilipennis Hartig det. H.H. Evenhuis 1980], [Alloxysta pilipennis (Hartig, 1840) ㅇ M. Ferrer-Suay det. 2011] (ZSM).

\section{Paralectotypes}

GERMANY: 1 q [711 (red label)], [Paralectotype Xystus pilipennis Hartig, 1840 q (red label)], [Alloxysta pilipennis (Hartig, 1840) + M. Ferrer-Suay det. 2011] (ZSM); 4 q 9 [Paralectotype Xystus pilipennis Hartig, 1840 q (red label)], [Alloxysta pilipennis (Hartig, 1840) $q$ M. Ferrer-Suay det. 2011] (ZSM).

\section{Additional specimens $(11 \widehat{\jmath}, 41+\circ$ 우}

CZECH REPUBLIC: 1 [ [Moravia, Palava near Mikulov, $\approx 400$ m, 9 Aug. 1991, L. Masner, sweep] (CNCI C-264); 1 + [Moravia, Ladnice environs, 7-9 Aug. 1991, L. Masner riparian, forest] (CNCI C-272).

GERMANY: 1 [ [Hesse, Gelnhausen, Sep. 1967, MT, A.W. Steffan] (CNCIC-228); 5 q $q$ [Schwarzwald, Todtmoos environs, 6 Aug. 1984, L. Masner, screen sweeping] (CNCI C-235).

IRAN: 1 [Damavand, 40 km, E. of Tehran in orchard, 7-15 Jul. 1977, M. Cox] (BMNH). 1 [Tehran Prov., Shahdasht, 25 Jun. 1978, J.T. Huber] (CNCI C-317); 1 §, 1 우 [Karaj, 40 km W Tehran, 25-28 Jun. 1978, J.T. Huber, YPT] (CNCI C-316).

JAPAN: 2 우 [Aichi Shitara, Uradani, $900 \mathrm{~m}$, beech forest, 23-29 May 1994, K. Yamagishi, YPT] (CNCI C-187); 1 \& [Iwate, Mt. Hayachine, 21 Jun. 1989, M.J. Sharkey, sweep] (CNCI C-198); 1 q 
[Ibaraki, Tsukuba, NIAES, 14-21 Jul. 1989, M.J. Sharkey, PT] (CNCI C-191); 1 q [Kyushu, 700 m, Fukuoka, MT. Hiko, 10-21 Jul. 1989, MT, K. Takeno and M. Sharkey] (CNCI C-171); 1 \& [Hokkaido, Aizan, 800 m, 4 Jul. 1989, sweep, M.J. Sharkey] (CNCI C-186); 1 q [Fukuoka, Mt. Hiko, 10-21 Jul. 1989, Takeno and M. Sharkey] (CNCI C-154); 1 \& [Fukuoka, Mt. Hiko, 18-25 Sep. 1989, K. Takeno and M. Sharkey] (CNCI C-159); 1 q [Hokkaido, Furano, Exp. Forest, 43¹5' N, 142²0’ E, 9 Aug. 1996, 500 m, L. Masner, sweep] (CNCI C-166); 1 o [Fukuoka, Mt. Hiko, 12-29 May 1989, Takeno and Sharkey] (CNCI C-145); 1 q [Fukuoka, Mt. Hiko, 700 m, 28 Apr.-10 May 1989, M.J. Sharkey, MT] (CNCI C-139); 1 § [Hokkaido, Hidako Mts below Pyo Tan, 500 m, 14 Aug. 1996, L. Masner, S.S. J-12] (CNCI C-161); 1 q [Aichi, Midori -Ku, Nagoya city, 2 Jun. 1984, R. Baczyuski] (CNCI C-180); 1 đ

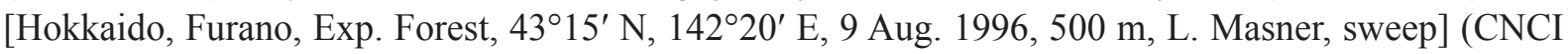
C-163); 1 [Aichi, Shitara, Uradani, 900 m, 13-19 May 1994, K. Yamagishi, EMT, beech forest] (CNCI C-223); 2 우 [Hokkaido, Sapporo, Jozankei, 350 m, 10-21 Aug. 1989, K. Maeto and M. Sharkey, MT] (CNCI C-206); 3 우우 [Aichi, Shitara, Uradani, 900 m, 2-8 May 1994, K. Yamagishi, EMT, beech forest] (CNCI C-224); 2 우우 [Iwate, Mt. Hayachine, 21 Jun. 1989, M. Sharkey, sweep] (CNCI C-208); 2 우 [Aichi, Shitara, Uradani, 900 m, 4-10 Jul. 1994, K. Yamagishi, EMT, beech forest] (CNCI C-217).

RUSSIA: 2 우 [Russian Far East, Primorski Krai Lazovski Zapovednik, c.170 Km, E. Vladivostok,

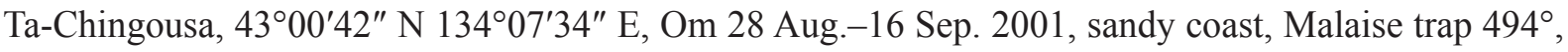
M. Quest coll., Alloxysta spp-indet dwarfs and intermediates] (BMNH B.M. 2009-59); 1 § [Primoskiy Kray Ussyriysk District, Gomotayozhnoye, 11-15 Aug. 2003, Malaise trap, M.V. Michailovskaya] (USNM); 1 ㅇ [Primorskiy Kray, Vladivostok environs, ??. ??.1992, A. Okulov] (CNCI C-297); 1 q [Magadanskaya Oblast, Aborigen Field Station, 500 m, 25 Jul.-10 Aug. 1990, D.M. Wood] (CNCI C-298).

SWEDEN: 1 ○ [Sk., Lund Zoological Mus, grounds, Aug. 1976] (BMNH).

SWITZERLAND: 6 ふึึึ, 3 우 [Dielsdorf, 650 m, 17 Aug. 1984, L. Masner, sweep] (CNCI C-290).

UNITED KINGDOM: England: 1 q [Surrey, Box Hill (near Dorking) 28 Aug. 1982, E.E. Grissell herbaceous vegetation] (USNM).

\section{Distribution}

Holarctic and Neotropical.

Certain records: Colombia (Ferrer-Suay et al. 2012f: 325), Germany (Hartig 1841: 352), Iran (FerrerSuay et al. 2013a: 39), Spain (Ferrer-Suay et al. 2013g: 327).

Uncertain records: Austria (Giraud 1860: 129; Hellén 1963: 16), England (Dalla Torre \& Kieffer 1910: 282), Finland (Hellén 1963: 16), France (De Gaulle 1908: 26; Dalla Torre \& Kieffer 1910: 282), Israel (Argaman 1988: 115), Poland (Kierych 1979b: 16), Norway (Hellén 1966: 393), Romania (Ionescu 1969: 249), Scotland (Cameron 1886: 85), Sweden (Thomson 1862: 407), Switzerland (Hellén 1963: 16), The Netherlands (Hellén 1963: 16), USA (Andrews 1978: 61).

New records: Czech Republic, Iran, Japan and Russia.

Alloxysta pleuralis (Cameron, 1879)

Figs 3.4, 5.8

Allotria pleuralis Cameron, 1879: 113. Type: deposited in BMNH (examined). 
Allotria (Allotria) pleuralis - Dalla Torre \& Kieffer 1902: 40.

Charips (Charips) pleuralis - Dalla Torre \& Kieffer 1910: 279.

Alloxysta pleuralis - Andrews 1978: 88.

\section{Diagnosis}

This species is easily differentiated from other species of Alloxysta by the following combination of features: a partially open radial cell (Fig. 5.8); the presence of pronotal carinae; two well-defined propodeal carinae that reach the posterior margin independently; female antenna with F1 longer than F2, F2 shorter than F3, and F3 shorter than F4 (Fig. 3.4); male antenna with F1-F3 subequal in length and slightly curved.

\section{Material examined}

\section{Lectotype}

UNITED KINGDOM: Scotland: + [8], [Cameron 96-76 (in front)], [pleuralis (behind, handwritten)], [Lectotype (round label with blue margins)], [Lectotype + Allotria pleuralis Cameron det. J. Quinlan, 1973], [B.M. Type Hym. 7. 122], [Alloxysta pleuralis (Cameron, 1879) \&, M. Ferrer-Suay det. 2012 (white label)] (BMNH).

\section{Paralectotype}

UNITED KINGDOM: Scotland: 1 \& [Cameron 96-76], [Paralectotype (round label with blue margins)],

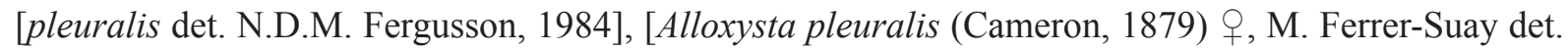
2012 (white label)] (BMNH).

Additional specimens ( $3 \widehat{\diamond}, 14 q \uparrow)$

CZECH REPUBLIC: 1 \% [central Bohemia, 30 Jul. 1991, J. Macek] (CNCI C-274).

GERMANY: 1 \& [Harz Mts-Brocken Dist., 4 Jul. 1927, H.T. Pagden, A. pleuralis det. N.D.M. Fergusson] (BMNH); 1 ठ [Rhine Valley near Hügelheim, 4750'10" N 7³7'9" E, 15 Jun. 1999, H. Goulet, sweeping old alfalfa] (CNCI C-230); 1 \& [Schwarzwald, Todtmoos environs, 6 Aug. 1984, L. Masner, screen sweeping] (CNCI C-235).

IRAN: 2 $q$ [Karaj, 40 km W Tehran, 25-28 Jun. 1978, J.T. Huber, YPT] (CNCI C-316); 1 q [Karaj, 40 km W Tehran, 3 Jul. 1978, J.T. Huber sweep] (CNCI C-315).

JAPAN: 1 [Tsukuba, Ibaraki, 21 Jun. 1987, sweep, C.M. Yoshimoto] (CNCI C-182).

NETHERLANDS: 1 ठ [BAA-99, 1961, Wageningen, J.P.O boomgaard op appel, als luis verzameld 24 Jun. 1961, mummie: 26 Jun., uitgekomen 10 Jul. 1961, mummie van Aphis pomi, 14, Alloxysta $\widehat{~}$ sp. det. J. Quinlan 1962] (handwritten) (BMNH); 1 ? [Groesveld Netherlands, 24 Aug. 1961, leg. H.H. Evenhuis, aphid mummy on cultivated apple, Alloxysta pleuralis (Cameron) det. H.H. Evenhuis 1979] (handwritten) (BMNH); $1 \widehat{\delta}$ [Oosterbeek, Netherlands, 11 Aug. 1964, leg. J. Norlander, aphid mummy on cultivated Cotoneaster pendula, Alloxysta pleuralis (Cameron) det. H.H. Evenhuis 1979] (BMNH) (handwritten).

SWEDEN: 1 q [Sk., Lund Zoological Mus. grounds, Aug. 1976] (BMNH).

SWITZERLAND: 5 우 [Dielsdorf, 650 m, 17 Aug. 1984, L. Masner, sweep] (CNCI C-290).

\section{Distribution}

Palaearctic. 
Certain records: England (Müller et al. 1999: 346), Scotland (Cameron 1886: 85; Fergusson 1986: 19), Serbia and Slovenia (Ferrer-Suay et al. 2013b: 358).

Uncertain records: England (Cameron 1879: 113; Fergusson 1986: 19), France (Kieffer 1902a: 14; De Gaulle 1908: 26; Gautier 1921: 305), Germany (Hübner et al. 2002: 507), India (Ahmad \& Singh 1996: 26), Ireland (Fergusson 1986: 19), Israel (Argaman 1988: 115), Norway (Westrum et al. 2010), Poland (Barczak 1991: 87), Spain (Tizado \& Nuñez-Perez 1993: 97).

New records: Czech Republic, Iran, Japan, Sweden and Switzerland.

\section{Alloxysta postica (Hartig, 1841)}

Figs 3.5, 5.9

Xystus posticus Hartig, 1841: 352. Type: deposited in ZSM (examined).

Allotria posticus - Taschenberg 1866: 130.

Allotria postica - Cameron 1890: 234.

Dilyta posticus - Kieffer 1900: 114.

Alloxysta (Alloxysta) postica - Dalla Torre \& Kieffer 1902: 39.

Alloxysta postica - Andrews 1978: 88.

\section{Diagnosis}

Alloxysta postica is mainly characterized by a partially open radial cell that is 2.5 times as long as wide (Fig. 5.9), the presence of pronotal carinae and propodeal carinae, female antenna with rhinaria beginning on F4, and pedicel-F4 subequal in length (Fig. 3.5). It is similar to A. citripes, but they can be differentiated by the shape of the propodeal carinae (clearly visible and forming a protruding plate in A. postica while not protruding in A. citripes) and the size of the radial cell (2.5 times as long as wide in A. postica (Fig. 5.9), but 2.1 times in A. citripes (Fig. 4.11)).

\section{Material examined}

\section{Lectotype}

GERMANY: $ᄋ$ [o], [lectotype H.H. Evenhuis (orange label)], [Xystus posticus Hartig det. H.H. Evenhuis 1980], [Alloxysta postica (Hartig, 1841) o M. Ferrer-Suay det. 2011] (ZSM).

\section{Additional specimens ( $3 \hat{\jmath} \widehat{\partial}, 38$ 웅)}

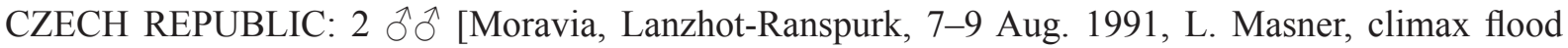
forest] (CNCI C-265); 1 ô, 1 ㅇ [Moravia, Lanzhot-Ranspurk, 7-9 Aug. 1991, L. Masner sweep, climax flood forest] (CNCI C-269); 7 우 [Moravia, Lanzhot-Ranspurk, 9-12 Aug. 1991, L. Masner, climax hardwood forest, PT] (CNCI C-270).

GERMANY: 1 q [Schwarzwald, Todtmoos environs, 6 Aug. 1984, L. Masner, screen sweeping] (CNCI C-235).

JAPAN: 1 q [Aomori, Tawodako, Kurowori, 40³0’ N, 14057’ E, 22-23 Aug. 1996, 800 m, L. Masner, YPT, forest] (CNCI C-157); 1 q [Hokkaido, Horoka, 800 m, 5 Jul. 1989, sweep, M.J. Sharkey] (CNCI C-152); 1 + [Hokkaido, Sapporo, Wisumai, 100 m, 30 Jun. 1989, M.J. Sharkey, sweep] (CNCI C-194); 1 앙 [Hokkaido, Sapporo, Jozankei, 350 m, Aug. 1989, K. Maeto and M. Sharkey, MT] (CNCI C-203); 1 ㅇ [Ibaraki, Tsukuba, NIAES, 27 Oct. 13 Nov. 1989, PT, M. Sharkey] (CNCI C-200); 1 ? [Ibaraki, Tsuchiura, Shishizuka-oike, 27 Oct. 13 Nov. 1989, M.J. Sharkey, MT] (CNCI C-192); 1 ㅇ [Fukuoka, Mt. Hiko, 700 m, 28 Apr.-10 May 1989, M.J. Sharkey, MT] (CNCI C-139); 1 \& [Honshu, Iwate, Iwaizuwi, 
Hitsutori, 770 m, 11-17 Aug. 1991, A. Smetana (J47)] (CNCI C-210); 1 q [Aichi, Shitara, Uradani, 900 m, 11-17 Jul. 1994, K. Yamagishi, EMT, beech forest] (CNCI C-222); 1 ㅇ [Aichi, Shitara, Uradani, 900 m, 13-19 Jun. 1994, K. Yamagishi, EMT, beech forest] (CNCI C-225); 1 ठิ [Honshu, Iwate, Kawai, Yoshibezawa, 1050 m, 12-17 Aug. 1991, A. Smetana (J50)] (CNCI C-212).

RUSSIA: 18 q [Primoskiy Kray Ussyriysk District, Gomotayozhnoye, 11-15 Aug. 2003, Malaise trap, M.V. Michailovskaya] (USNM); 1 q [Primorskiy Kray, Vladivostok environs, ??. ??.1992, A. Okulov] (CNCI C-297).

\section{Distribution}

Palaearctic.

Certain records: France (Ferrer-Suay et al. 2015a), Germany (Hartig 1841: 352).

Uncertain records: Bulgaria and Balkan Peninsula (Vasileva-Sumnalieva 1976: 23), Czech Republic (Dalla Torre \& Kieffer 1910: 257).

New records: Japan and Russia.

Alloxysta proxima Belizin, 1962

Figs 3.6, 5.10

Alloxysta proxima Belizin, 1962: 128. Type: deposited in ZIN (examined).

\section{Diagnosis}

Alloxysta proxima is mainly characterized by a completely open radial cell that is 2.3 times as long as wide (Fig. 5.10); the absence of pronotal propodeal carinae; and female antenna with the rhinaria beginning on F4, F1 longer than pedicel, F2 longer than F1, F2 longer than F3, and F3 subequal to F4 (Fig. 3.6). The combination of these features means that no other known species of Alloxysta is similar to A. proxima.

\section{Material examined}

\section{Holotype}

RUSSIA: ㅇ [Preobrazhenskoe, O. Mednyi, K. Gorodkov, 1959], [Holotypus Alloxysta proxima m V. Belizin det 9 (red label)], [Alloxysta proxima Belizin, 1962 M. Ferrer-Suay det. 2011] (ZIN).

\section{Distribution}

Palaearctic.

Certain records: Medny Island (Preobrazenskoe, Russia) (Belizin 1962: 128), France (Ferrer-Suay et al. 2015a).

Alloxysta pseudoconsobrina Ferrer-Suay, 2017

Alloxysta pseudoconsobrina Ferrer-Suay, 2017: 430. Type: deposited in BMNH.

\section{Diagnosis}

Alloxysta pseudoconsobrina is characterized as a brachypterous species very similar to A. curta. They can be differentiated by the presence of propodeal carinae (present in A. curta, but absent in 
A. pseudoconsobrina) and the F1-pedicel proportion (F1 is subequal to pedicel in A. curta, but longer than pedicel in A. pseudoconsobrina).

\section{Material examined}

Holotype

UNITED KINGDOM: England: $\lesssim$ [Surrey: Barnes Common: 12 Sep. 2009: J.S. Noyes, BMNH(E) 2008-118] (BMNH B.M. 2008-118).

\section{Distribution}

England (Ferrer-Suay et al. 2017).

Alloxysta pusilla (Kieffer, 1902)

Figs 3.7, 5.11

Allotria (Allotria) pusilla Kieffer, 1902: 13. Type: deposited in NHMA(Dessart 1969: 192, not examined).

Charips (Charips) pusillus pusillus - Dalla Torre \& Kieffer 1910: 279.

Alloxysta pusilla pusilla - Andrews 1978: 89.

\section{Diagnosis}

Alloxysta pusilla is mainly characterized by a closed radial cell that is 2.7 times as long as wide in females (Fig. 5.11) and 2.4 times in males; the presence of pronotal and propodeal carinae that form a plate; female antenna with rhinaria beginning on F3, F1 longer than pedicel and F2, F2 shorter than F3, and F3 shorter than F4 (Fig. 3.7); male antenna with rhinaria beginning on F1, pedicel-F3 subequal in length, F4 longer than F3, and F1-F3 slightly curved. It is similar to A. pilipennis, but can be differentiated by the proportions of the flagellomere in females (F2 is shorter than F3 in A. pusilla females (Fig. 3.7), but subequal to $\mathrm{F} 3$ in A. pilipennis females (Fig. 3.3)), flagellomere proportions and shape in males (F1-F3 are subequal in length and slightly curved in A. pusilla males, but F1-F3 are not subequal and not curved in A. pilipennis males), and the size of the radial cell (2.7 times as long as wide in A. pusilla females (Fig. 5.11), but 2.4 times in A. pilipennis females (Fig. 5.7)).

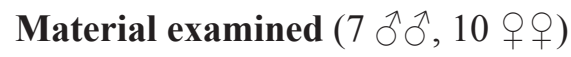

CZECH REPUBLIC: 1 \& [Moravia, Dyje River, near Znojmo, 12 Aug. 91, L. Masner] (CNCI C-263).

GERMANY: 1 [Wern, Riedenerwald, 15 Aug. 1978, L. Huggert, sweep, in open oak wood] (CNCI

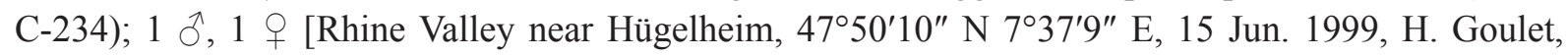
sweeping old alfalfa] (CNCI C-230).

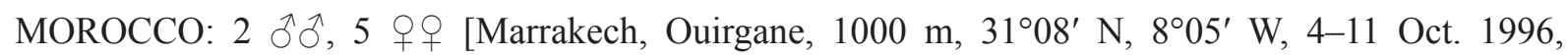
C. Kassebeer, MT] (CNCI C-279).

NETHERLANDS: 1 ○े [Wageningen, 26 Jul. 1978, L. Huggert, swept in marsh] (CNCIC-310).

PORTUGAL: 1 § [Madeira, June 1935, G.V. Vredenburg, Brit. Mus. 1935-615] (BMNH).

TURKEY: 2 ふð, 2 qq [Bornova-Izmir, 4 May 1976, E. Fakültesi (handwritten), Ex. Myzus persicae] (BMNH). 


\section{Distribution}

Palaearctic and Oriental.

Certain records: Andorra (Ferrer-Suay et al. 2011: 358), England (Sanders \& van Veen 2010: 706), France (Kieffer 1902a: 13; De Gaulle 1908: 26), Japan, Thailand and Taiwan (Ferrer-Suay et al. 2013i).

Uncertain records: Germany (Hübner et al. 2002: 507), Italy (Mantero 1906), Romania (Ionescu 1969: 255).

New records: Czech Republic, Morocco, The Netherlands, Portugal and Turkey.

Alloxysta quedenfeldti (Kieffer, 1909)

Figs 3.8, 5.12

Charips quedenfeldti Kieffer, 1909: 482. Type: deposited in ZMHB (examined).

Alloxysta quedenfeldti - Andrews 1978: 89.

\section{Diagnosis}

Alloxysta quedenfeldti is mainly characterized by a closed radial cell that is 2.8 times as long as wide (Fig. 5.12); the absence of pronotal and propodeal carinae; and female antenna with rhinaria beginning on F3, F1 subequal to pedicel, and F1-F3 subequal in length (Fig. 3.8). It is similar to A. aperta, but differs in the proportion of the pedicel-F1 (F1 is subequal to pedicel in A. quedenfeldti (Fig. 3.8), but longer than pedicel in A. aperta (Fig. 2.2)) and where rhinaria begin (on F3 in A. quedenfeldti (Fig. 3.8), but on $\mathrm{F} 4$ in $A$. aperta (Fig. 2.2)).

\section{Material examined}

\section{Holotype}

ALGERIA: $\widehat{\partial}$ [Blidah-Médéah, Algerien, Juli-August 84 Quedenfeldt(blue label)], [Charips quedenfeldti K. (handwritten)], [holotype H.H. Evenhuis 1984 (red label)], [Alloxysta quedenfeldti (Kieffer) ô det. H.H. Evenhuis 1904], [Zool. Mus. Berlin] (ZMHB).

\section{Distribution}

North Africa.

Certain records: Algeria (Kieffer 1909: 482).

Alloxysta ramulifera (Thomson, 1862)

Figs 3.9, 5.13

Allotria ramulifera Thomson, 1862: 407. Type: deposited in MZLU (examined).

Allotria (Allotria) ramulifera - Dalla Torre \& Kieffer 1902: 40.

Charips (Charips) ramulifera - Dalla Torre \& Kieffer 1910: 281.

Alloxysta ramulifera - Hellén 1963: 20.

\section{Diagnosis}

Alloxysta ramulifera is mainly characterized by a small closed radial cell that is 2.0 times as long as wide (Fig. 5.13); the presence of pronotal carinae that are very small and sometimes difficult to see 
under the pubescence; propodeal carinae that form a plate; and antenna with rhinaria and club shape beginning on F4, F1 subequal to pedicel, F1 longer than F2, F2 subequal to F3, and F3 shorter than F4 (Fig. 3.9). Alloxysta ramulifera is very similar to A. arcuata, both species having pronotal carinae, propodeal plates, and a small and closed radial cell. They can be distinguished by the shape of the pronotal carinae (small and sometimes very difficult to see under the pubescence in A. ramulifera, but thick and clearly visible in A. arcuata), the shape of propodeal plate (in A. ramulifera the carinae are straight, are separated by setae in the first third, and form a plate in the last two thirds, while they form a complete plate in A. arcuata), and the size of the radial cell (2.0 times as long as wide in A. ramulifera (Fig. 5.13), but 2.3 times in A. arcuata (Fig. 4.3)).

\section{Material examined}

\section{Lectotype}

SWEDEN: $q$ [Årefkutan, 1840. 18.10 (handwritten)], [All. ramulifera. n. $q$ (handwritten)], [lectotype H.H. Evenhuis (orange label)], [Allotria ramulifera Thomson, det. H.H. Evenhuis, 1978], [ZML 2000, 007 (green label)], [Alloxysta ramulifera (Thomson, 1862) 우 M. Ferrer-Suay det. 2011] (MZLU).

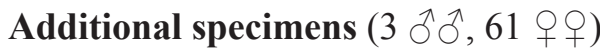

DENMARK: 7 + $q$ [W. Zealand, Gyrstinge, $20 \mathrm{~km} \mathrm{~S}$ of Holbaek, 28 Aug. 1994] (CNCI C-243); 1 [S. Zealand, Karrebaeksminde, 28 Aug. 1994, edge of salt water bay] (CNCI C-242).

FRANCE: 4 우 [Pyrénées Orientales, Arles-sur-Tech, D. and J. Clark, 25 Jun. 1961] (BMNH B.M. 1962-149); 1 \& [B. du Rhone Fonscolombe (3), 2 Jul. 1980, M.W.R. de V. Graham coll.] (BMNH B.M. 1995-489).

GERMANY: 2 $ð$, 2 qq [Göltingen, 29 Aug. 1959, H. Prilop Ex Dorilas fabae, C.I.E., coll. no. 16782] (BMNH); 1 \& [Baden Waldkirch, A. Koebele B.M. 1942-95] (BMNH B.M. 1942-95).

IRAN: 1 ภ, 1 ㅇ [Karaj, 40 km W Tehran, 3 Jul. 1978, J.T. Huber sweep] (CNCI C-315).

ITALY: 1 ㅇ [(Lago) Bolsena, 27 Aug. 1972, Boucek] (BMNH).

MOROCCO: 1 ㅇ [Marrakech, Ouirgane, 1000 m, 31 ${ }^{\circ} 08^{\prime}$ N, $8^{\circ} 05^{\prime}$ W, 4-11 Oct. 1996, C. Kassebeer, MT] (CNCI C-279).

NETHERLANDS: 2 우 [Laersum, Broekhuizen, Vinca minor vegetation, Mal. trap., June 1976, Leg. H.J. Vlug, Netherlands, + , Alloxysta minuta (Hartig) det. H.H. Evenhuis, 1978] (BMNH).

RUSSIA: 1 [ [Russian Far East, Primorski Krai Lazovski Zapovednik, c.170 Km E. of Vladivostok,

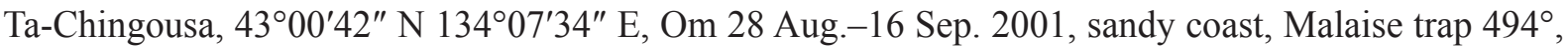
M. Quest coll., Alloxysta spp-indet dwarfs and intermediates] (BMNH B.M. 2009-59).

SERBIA: 1 ㅇ [Srbija Drazevac, nr. Belgrade, 27-28 Jun. 1981, M. Day and M. Fitton] (BMNH); 1 ㅇ [Srbija Drazevac, nr. Belgrade, 27-28 Jun. 1981, M. Day and M. Fitton] (BMNH).

SWEDEN: 31 $q$ [sk. kivik, 19 Jul. 1938, d.m.s.p. and j.f.p.] (BMNH B.M. 1938-414); 1 [ [Uppsala, Hogadalen, 10-17 Aug. 1990, Malaise trap, F. Ronquist] (CNCI C-305); 3 웅 [Uppsala, Hogadalen, 16-10 Aug. 1990, Malaise trap, F. Ronquist] (CNCI C-307); 1 ㅇ [Uppsala, Hogadalen, 4-16 Sep. 1990, S: UP, Malaise trap leg, F. Ronquist, CYNIPOIDEA det. K. bolte] (CNCI C-308).

SWITZERLAND: 3 우 [Dielsdorf, 650 m, 17 Aug. 1984, L. Masner, sweep] (CNCI C-290). 


\section{Distribution}

Palaearctic.

Certain records: England (Müller et al. 1999: 346), Madeira (Borges et al. 2008; Ferrer-Suay et al. 2012g: 12), Spain (Ferrer-Suay et al. 2013g), Sweden (Thomson 1862: 408).

Uncertain records: Austria (Giraud 1860: 127; Hellén 1963: 21), Belgium (Lameere 1907), England (Andrews 1978: 86), Finland (Hellén 1963: 21), France (Kieffer 1902b: 601; De Gaulle 1908: 26), Germany (Hartig 1840: 200; Förster 1869: 340; Hübner et al. 2002: 507), Hungary (Dalla Torre \& Kieffer 1910: 277), Israel (Argaman 1988: 115), Poland (Kierych 1979b: 15), Romania (Ionescu 1969: 235; Prelipcean et al. 2004: 60), Scotland (Cameron 1886: 86), The Netherlands (Hellén 1963: 21).

New records: Denmark, Iran, Morocco, Russia and Switzerland.

Alloxysta rufiventris (Hartig, 1840)

Figs 3.10, 5.14

Xystus rufiventris Hartig, 1840: 200. Type: deposited in ZSM (examined).

Allotria rufiventris - Taschenberg 1866: 130.

Dilyta rufiventris - Kieffer 1900: 114.

Alloxysta (Alloxysta) rufiventris - Dalla Torre \& Kieffer 1902: 39.

Alloxysta rufiventris - Andrews 1978: 90.

\section{Diagnosis}

Alloxysta rufiventris is mainly characterized by a partially open radial cell that is 2.2 times as long as wide (Fig. 5.14); the absence of pronotal carinae; the presence of propodeal carinae that form a plate; and antenna with rhinaria and club shape beginning on F4 and pedicel-F4 subequal in length (Fig. 3.10). According to these features, there is no other species of Alloxysta similar to A. rufiventris.

\section{Material examined}

\section{Lectotype}

GERMANY: $\widehat{\delta}[\hat{\jmath}]$, [lectotype H.H. Evenhuis (orange label)], [Xystus rufiventris Hartig det. H.H. Evenhuis 1980], [Alloxysta rufiventris (Hartig, 1840) ${ }^{\top}$ M. Ferrer-Suay det. 2011] (ZSM).

\section{Distribution}

Palaearctic.

Certain records: Germany (Hartig 1840: 200).

Alloxysta salicicola Belizin, 1973

Figs 3.11, 5.15

Alloxysta salicicola Belizin, 1973: 36. Type: deposited in ZIN (examined).

\section{Diagnosis}

Alloxysta salicicola is mainly characterized by a partially open radial cell that is 2.6 times as long as wide; the presence of pronotal carinae; smooth propodeum; and antenna with rhinaria and club shape beginning on F3, F1 longer than pedicel and F2, and F2 subequal to F3. It is similar to A. macrophadna, 
but can be easily differentiated by flagellomere proportions (F2-F4 are subequal in A. salicicola (Fig. 3.11), but not subequal in A. macrophadna (Fig. 2.25)) and the size of the radial cell (2.6 times as long as wide in $A$. salicicola (Fig. 5.15) but 3.3 times in A. macrophadna (Fig. 4.21)).

\section{Material examined}

\section{Holotype}

RUSSIA: + [Severo-Kurilsk, Paramushir I., aphids on Salix, 5 Aug. 1964 (red label)], [Holotypus Alloxysta salicicola m V. Belizin det $\supsetneq$ (red label)], [Alloxysta salicicola Belizin, 1973 ㅇ M. FerrerSuay det. 2011] (ZIN).

\section{Distribution}

Palaearctic.

Certain records: Russia (Belizin 1973: 36), Slovenia (Ferrer-Suay et al. 2013b: 359).

\section{Alloxysta sawoniewiczi (Kierych, 1988)}

Figs 3.12, 5.16

Adelixysta sawoniewiczi Kierych, 1988: 351. Type: deposited in MZPW (Kierych 1988: 353) (not examined).

Alloxysta sawoniewiczi - Menke \& Evenhuis 1991: 150.

\section{Diagnosis}

Alloxysta sawoniewiczi is closely related to A. arcuata. Both have a closed radial cell, pronotal and propodeal carinae, and F1 subequal to pedicel. They can be distinguished by the length of the antenna (A. sawoniewiczi has antenna shorter than body length, while in A. arcuata it is longer), where rhinaria begin (in A. sawoniewiczi on F5 in females (Fig. 3.12) and on F4 in males, while in A. arcuata on F3 in females (Fig. 2.4) and F2 in males), and the shape of the propodeal carinae (in A. sawoniewiczi, they are narrow and well-defined in the anterior half and wide and forming a plate in the posterior half with sharp margins, while in A. arcuata they form a complete plate with few setae on top and margins slightly curved).

Material examined $(1 \hat{\jmath}, 12$ + $q)$

JAPAN: 1 + [Aichi Shitara, Uradani, 900 m, beech forest, 23-29 May 1994, K. Yamagishi, YPT] (CNCI C-187); 1 q [Kyushu, Fukuoka, Mt. Hiko, 700 m, 9-10 May 1989, M.J. Sharkey, sweep] (CNCI C-138); 1 q [Fukuoka, Mt. Hiko, 700 m, 9-10 May 1989, M.J. Sharkey, sweep] (CNCI C-183); 1 q [Kyushu, Fukuoka, Mt. Hiko, 700 m, 18-25 Aug. 1989, K. Takeno and M. Sharkey, MT] (CNCI C-185); 1 q [Hokkaido, Sapporo, Jozankei, 350 m, 20-31 Jul. 1989, K. Maeto and M. Sharkey] (CNCI C-140); 1 [Hokkaido, Sapporo, Jozankei, 350 m, 12-21 Sep. 1989, K. Maeto and M. Sharkey, MT] (CNCI C-205); 3 q $q$ [Ibariki, Tsukuba, NIAES, 13 Nov.-22 Dec. 1989, M.J. Sharkey, PT] (CNCI C-142); 1 ठૈ, 1 q [Tsukuba, Ibaraki, 21 Jun. 1987, C.M. Yoshimoto, sweep] (CNCI C-182); 2 우 [C-207, Hokkaido, Sapporo, Jozankei, 350 m, 29 Aug.-12 Sep. 1989, K. Maeto and M. Sharkey, MT] (CNCI C-207).

\section{Distribution}

Palaearctic.

Certain records: Poland (Kierych 1988: 351), Nepal, Thailand and Taiwan (Ferrer-Suay et al. 2013i).

New record: Japan. 
Alloxysta semiaperta Fergusson, 1986

Figs 3.13, 5.17

Alloxysta semiaperta Fergusson, 1986: 11. Type: deposited in BMNH (examined).

\section{Diagnosis}

Alloxysta semiaperta is mainly characterized by a partially open radial cell that is 2.7 times as long as wide (Fig. 5.17); the presence of pronotal carinae; the absence of propodeal carinae; and antenna with rhinaria and club shape beginning on F3 in females and F4 in males, F1 longer than pedicel and F2, F2 shorter than F3 in females and subequal in males, and F3 shorter than F4 (Fig. 3.13). It is similar to A. salicicola, but can be differentiated by the length-to-width ratio of the flagellomeres, which is greater in A. salicola.

\section{Material examined}

\section{Holotype}

UNITED KINGDOM: England: $\$$ [Oxon, 1 Sep. 1977, L.A. Mound Ex Epilobium], [Holotype (round label with red margin)], [Holotype of Alloxysta semiaperta det. N.D.M. Fergusson, 1984], [B.M. Type Hym. 7. 174] (BMNH B.M. Type Hym. 7. 174).

\section{Paratype}

UNITED KINGDOM: England: $\widehat{\jmath}$ [Oxon, 1 Sep. 1977], [L.A. Mound, Ex. Epilobium (handwritten)], [§], [Paratype (round label with yellow margin); A. semiaperta det. N.D.M. Fergusson] (BMNH).

Additional specimens $(1 \hat{\rho}, 7$ 우)

SWEDEN: 1 ð, 7 우 [Sk. Kivik, 19 Jul. 1938, D.M.S.P and J.F.P.] (BMNH B.M. 1938-414).

\section{Distribution}

Palaearctic.

Certain records: England (Fergusson 1986: 19).

Uncertain records: Ireland (O’Connor \& Nash 1997).

New record: Sweden.

Alloxysta slovenica Ferrer-Suay \& Pujade-Villar, 2013

Figs 3.14, 5.18

Alloxysta slovenica Ferrer-Suay et al., 2013b: 259. Type: deposited in UB (examined).

\section{Diagnosis}

Alloxysta slovenica is mainly characterized by a partially open radial cell that is 2.2 times as long as wide; absence of pronotal carinae; propodeal carinae that form a plate; and antenna with rhinaria and club shape beginning on F4, F1 shorter than pedicel and F2, F2 slightly longer than F3, and F3 shorter than F4. There is no other known Alloxysta species closely similar to A. slovenica.

\section{Material examined}

Holotype

SLOVENIA: + [pinned. Original label: VP3 (handwritten), Alloxysta sp. det. A. Stojanović. 2011] (UB). 
Additional specimens (1 $q$ )

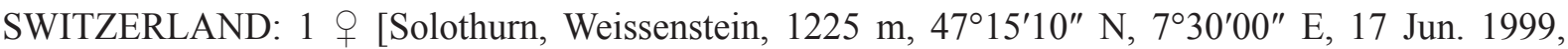
Goulet/White, lush meadow] (CNCI C-285).

\section{Distribution}

Palaearctic.

Certain records: Slovenia (Ferrer-Suay et al. 2013b: 361).

\section{Alloxysta soluta Hellén, 1963}

Figs 3.15, 5.19

Alloxysta soluta Hellén, 1963: 10. Type: deposited in MZH (examined).

\section{Diagnosis}

Alloxysta soluta is mainly characterized by a partially open radial cell that is 2.3 times as long as wide (Fig. 5.19), the absence of pronotal and propodeal carina, and antenna with rhinaria and club shape beginning on F4 and F1 longer than pedicel (Fig. 3.15). There is no other known Alloxysta species closely related.

\section{Material examined}

\section{Lectotype}

FINLAND: + [Jomala], [Fennia], [Hellén], [2313], [typus (red label)], [Alloxysta soluta Hell. (handwritten)], [Mus. Zool. Helsinki Loan No. HY 4113 (yellow label)], [Mus. Zool. Helsinki Loan No. HY 2012 - 1832 (yellow label)], [Lectotype Alloxysta soluta Hellén, 1863 q desig. M. Ferrer-Suay 2012 (red label)], [Alloxysta soluta Hellén, 1963 M. Ferrer-Suay det. 2012] (MZH).

\section{Paralectotype}

FINLAND: q [Jomala], [Fennia], [Hellén], [2645], [soluta m (handwritten)], [typus (red label)], [Mus. Zool. Helsinki Loan No. HY 2012 - 1829 (yellow label)], [Paralectotype Alloxysta soluta Hellén, 1863 우 M. Ferrer-Suay 2012 (red label)], [Alloxysta soluta Hellén, 1963 ․ M. Ferrer-Suay det. 2012] (MZH).

\section{Distribution}

Palaearctic.

Certain records: Finland (Hellén 1963: 11).

\section{Alloxysta tscheki (Giraud, 1860)}

Figs 3.16, 5.20

Allotria tscheki Giraud, 1860: 128. Type: deposited in NMW (examined).

Allotria (Allotria) tscheki - Dalla Torre \& Kieffer 1902: 41.

Charips (Charips) tscheki - Dalla Torre \& Kieffer 1910: 285.

Charips tscheki-Dunn 1949: 106.

Alloxysta tscheki-Hellén 1963: 18. 


\section{Diagnosis}

Alloxysta tscheki is morphologically very similar to A. consobrina and it is practically impossible to differentiate them under the microscope (see comments).

\section{Material examined}

\section{Lectotype}

AUSTRIA: $\hat{\jmath}$ [All. Tscheki det. Giraud Type (handwritten)], [Ex. Aphidibus; Ribes rubr. (handwritten)], [Allotria Tscheki Giraud (handwritten)], [Allotria Tscheki Giraud 2ふへર, det. H.H. Evenhuis, 1976 (handwritten, orange label)], [das rechte Männchen wäre als Lektotypus zu wählen, H.H. Evenhuis (handwritten, orange label)], [NHMW (yellow label)], [LECTOTYPE Allotria tscheki Giraud, $1860 \overbrace{}^{\Uparrow}$ Desig. H.H. Evenhuis, 1976 (red label)], [Alloxysta tscheki (Giraud, 1860) §̊ det. M. Ferrer-Suay 2013] (NMW).

\section{Paralectotypes}

AUSTRIA: $1 \hat{\jmath}$ [Tscheki, Pirstiny, 1867 (handwritten)], [All. Tscheki det. Giraud Type (handwritten)], [Allotria Tscheki Giraud, $2 \widehat{\jmath} \widehat{\jmath}$, det. H.H. Evenhuis, 1976 (handwritten)], [NHMW (yellow label)], [PARALECTOTYPE Allotria tscheki Giraud, $1860 \hat{\jmath}$ (red label)], [Alloxysta tscheki (Giraud, 1860) $\lambda$ det. M. Ferrer-Suay 2013] (NMW); 1 \& [All. Tscheki det. Giraud Type (handwritten)], [understandable, Ex. Aphis Ribes Tschek col.], [Allotria Tscheki Giraud 2 q 9 det. H.H. Evenhuis 1976 (handwritten, orange label)], [NHMW (yellow label)], [PARALECTOTYPE Allotria tscheki Giraud, $1860 \hat{\curvearrowright}$ (red label)], [Alloxysta tscheki (Giraud, 1860) o det. M. Ferrer-Suay 2013] (NMW); 2 우 [Type (handwritten)], [All. Tscheki det. Giraud Type (handwritten)], [Allotria Tscheki Giraud (handwritten)], [2우, det. H.H. Evenhuis, 1976 (handwritten, orange label)], [NHMW (yellow label)], [PARALECTOTYPE Allotria tscheki Giraud, 1860 $\widehat{o}$ (red label)], [Alloxysta tscheki (Giraud, 1860) $q$ det. M. Ferrer-Suay 2013] (NMW).

\section{Distribution}

Palaearctic.

Certain records: Austria (Giraud 1860: 128), Iran (Ferrer-Suay et al. 2013a: 39), Romania (Feraru et al. 2005: 67), Japan (Ferrer-Suay et al. 2013i).

Uncertain records: Austria (Giraud 1860: 128; Hellén 1963: 19), Belgium (Lameere 1907), England (Cameron 1889: 54), Finland (Hellén 1963: 19), France (De Gaulle 1908: 26; Dalla Torre \& Kieffer 1910: 285), Germany (Taschenberg 1866: 129; van Veen et al. 2003: 450), Hungary (Dalla Torre \& Kieffer 1910: 285), Scotland (Cameron 1886: 85), Western Europe (Andrews 1978: 91).

\section{Comments}

The morphological features of $A$. tscheki are very similar, if not identical, to those of $A$. consobrina. According to Van Veen et al. (2003), who treated A. consobrina as A. fuscicornis, these two species have to be considered as biologically different, because their typical hosts are different: A. consobrina tends to attack Brevicorine brassicae (L., 1758) through Diaretiella rapae (M'cIntosh, 1855), while A. tscheki appears in Cryptomizus sp. through Aphidius ribes Haliday, 1834. Additionally, taking into account the sequences of the ITS2 gene, these two species have different haplotypes. However, these arguments can be disputed. Firstly, Alloxysta consobrina is a cosmopolitan species that has already been found in a diverse range of hosts, although not yet in Aphidius ribes. Secondly, the molecular results are not conclusive due to the low number of specimens analyzed. Also, Van Veen et al. (2003) only took into account specimens collected in a reduced area and studied just five specimens of $A$. tscheki. For these reasons, we consider that intraspecific variability has not been studied thoroughly enough and that a complete study should be done in order to clarify the status of these two species. While awaiting a more comprehensive assessment, we treat these two species as separate (Ferrer-Suay et al. 2015b: 33). 
Alloxysta victrix (Westwood, 1833)

Figs 3.17, 5.21

Allotria victrix Westwood, 1833: 495. Type: deposited in OUMNH (Andrews, 1978: 92) (not examined).

Xystus victrix - Rondani 1878: 177.

Allotria (Allotria) victrix - Dalla Torre \& Kieffer 1902: 41.

Charips (Charips) victrix victrix - Dalla Torre \& Kieffer 1910: 285.

Charips victrix - Rohwer \& Fagan 1917: 360.

Alloxysta victrix - Hellén 1963: 16.

Alloxysta victrix victrix - Andrews 1978: 92.

\section{Diagnosis}

Alloxysta victrix is mainly characterized by a large closed radial cell that is 3.0 times as long as wide (Fig. 5.21); the presence of pronotal carinae; the absence of propodeal carinae; the lack of setae on longitudinal areas where carinae are present in other species of Charipinae (Fig. 9.2); and male and female antenna with rhinaria beginning on F3, F1 longer than pedicel and F2, F2-F4 subequal (Fig. 3.17), and F1-F3 curved in males. It is similar to A. consobrina, but can be differentiated by the proportions of the flagellomere (F2-F4 are subequal in length in A. victrix (Fig. 3.17), while F2 is subequal to F3 and $\mathrm{F} 3$ is shorter than F4 in A. consobrina (Fig. 2.14)), the size of the radial cell (3.0 times as long as wide in A. victrix (Fig. 5.21), but 2.7 times in A. consobrina (Fig. 4.12)), and the degree of propodeal pubescence (in A. victrix, the propodeum lacks setae at the longitudinal area where the carinae are present in other Charipinae, while in A. consobrina, the propodeum is completely covered with dense setae).

\section{Material examined ( $376 \hat{\jmath} \widehat{\partial}, 522$ q $q)$}

CYPRUS: 1 [Limassol, Feb. 1934, G.A. Mavromoustakis] (BMNH B.M. 1935-55); 1 đ, 2 우 [Limassol, 25 May 1934, G.A. Mavromoustakis] (BMNH B.M. 1935-55); 1 q [Kilani, Krios R., 11 Sep. 1937, G.A. Mavromoustakis] (BMNH B.M. 1937-808).

CZECH REPUBLIC: 1 [Bohemia: Praha-Kunratice, 18 Jun. 1964, P. Mikula] (BMNH); 6 우 [Moravia, Dyje River, near Znojmo, 12 Aug. 1991, L. Masner] (CNCI C-263); 1 o [central Bohemia, 30 Jul. 1991, J. Macek] (CNCI C-274); 15 $\widehat{\jmath} \widehat{\jmath}, 39$ 우 우 [Moravia, Palava near Mikulov, $\approx 400$ m, 9 Aug.

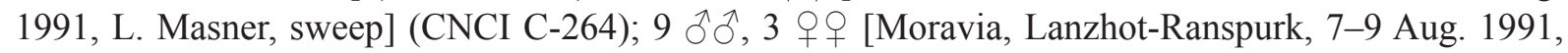

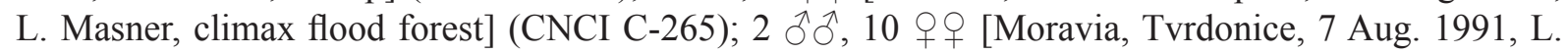

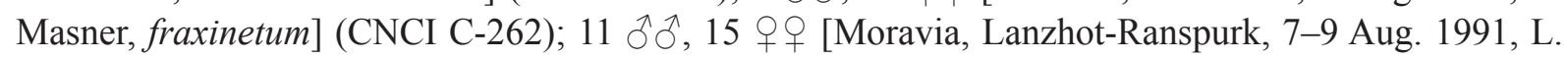
Masner, sweep, climax flood forest] (CNCI C-268); 2 ठิ $\widehat{\jmath}, 9$ 우우 [Moravia, $16 \mathrm{~km} \mathrm{~N}$ Blansko, 8 Aug.

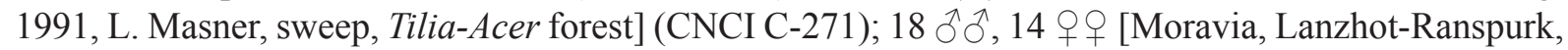
7-9 Aug. 1991, L. Masner, sweep, climax flood forest] (CNCI C-269); 1 ô, 7 우 [Moravia, Javorina,

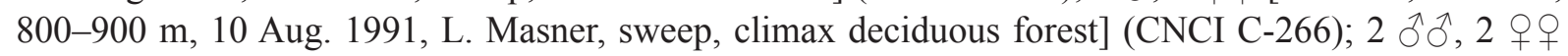
[Moravia, Lanzhot-Ranspurk, 9-12 Aug. 1991, L. Masner, climax hardwood forest, PT] (CNCI C-270); 1 [ [Moravia, Vranov, Dyje River, 13 Aug. 1991, L. Masner] (CNCI C-273); 13 $\widehat{\jmath}, 26 q q$ [Moravia, Ladnice environs 7-9 Aug. 1991, L. Masner, riparian forest] (CNCI C-272); 1 [Praha-Troja, 6-9 Sep. 1999, YPT, L. Masner, steppe] (CNCI C-284); 2 q $q$ [Moravia, Zidlochovice, 20 Nov. 1984, N.D. Springate] (BMNH).

DENMARK: 1 [W. Zealand, Gyrstinge, 20 km S of Holbaek, 28 Aug. 1994] (CNCI C-243).

FRANCE: 2 $q$ [Montpelier, 1980 Dufom (handwritten), Alloxysta victrix (Westwood) 9 det. J. Quinlan, 1987] (BMNH); 8 ठิ $\widehat{\jmath}, 10$ + $q$ [Pyrénées Orientalis nr. Arñes-sur. Tech, D. and J. Clark, 23 May 1961]

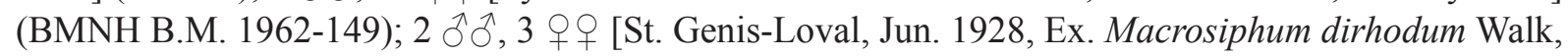


R. Pussard, Charips victrix Wstw, $q$ Ch. Ferriere det] (BMNH); 1 \& [St. Genis-Laval, Jun. 1928, Ex Macrosiphum dirhodum, Walk., R. Pussard, Charips victrix Westw., Ch. Ferriere det., Pres. by Imp. But. Ent.] (BMNH B.M. 1929-196); 1 ㅇ [B. du Rhone Fonscolombe (3), 2 Jul. 1980, M.W.R. de V. Graham

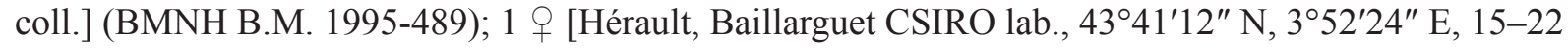
May 1993, P.G. Mason, champ sauvage, MT] (CNCI C-302); 2 우 [Haute Savoie, Bossy sur Frangy,

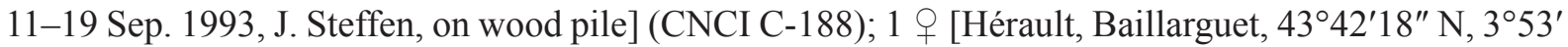
E, 12-24 Jun. 1995, P.G. Mason, pine look forest, PT and MT] (CNCI C-301); 2 + 9 [Haute Savoie, Bossy sur Frangy, 17-30 Aug. 1993, J. Steffen, on wood pile] (CNCI C-289); 1 q [Hérault, Montferrier sur Lez, 4340' N, 351' E, 23 May 1994, P.G. Mason, YPT] (CNCI C-300).

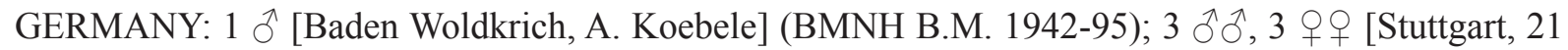
Jul. 1937, T.H. Rowsell and B.J. Clifton] (BMNH B.M. 1937-539); 1 §, 2 우 [Wern, Riedenerwald, 15 Aug. 1978, L. Huggert, sweep, in open oak wood] (CNCI C-234); 1 \& [Mainz, 18 Sep.-1 Oct. 1965, A.W. Steffan] (CNCI C-236); 1 [ [Munich, 23 Jul. 1984, R. Wharton] (CNCI C-239); 1 [ [Mainz, 26 Aug.-3 Sep. 1965, A.W. Steffan] (CNCI C-231); 2 우 [Mainz, 18-28 Jun. 1968, A.W. Steffan] (CNCI C-226); 1 으 [Ingelheim am Rhein, MT, 1 May-5 Jun. 1969, A.W. Steffan] (CNCI C-227); 1 $\lambda, 4$ 우 [Ingelheim am Rhein, ??.??.1968, MT in orchard, A.W. Steffan] (CNCI C-238); 6 우 [Ingelheim am Rhein, 1-30 Sep. 1968, MT, A.W. Steffan, orchard, Cynipoidea] (CNCI C-237); 2 우 [Mainz, 15 Nov. 1965, A.W. Steffan] (CNCI C-233); 15 ふ઼, 82 우 [Schwarzwald, Todtmoos environs, 6 Aug. 1984, L. Masner, screen sweeping] (CNCI C-235); 33 ㅅํ, 20 우우 [Anmuhle, near Hamburg Sachsenwald, 22 Aug. 1984, L. Masner, screen sweeping] (CNCI C-296).

IRAN: 1 [ [Karaj, 40 km W Tehran, 25-28 Jun. 1978, J.T. Huber, YPT] (CNCI C-316).

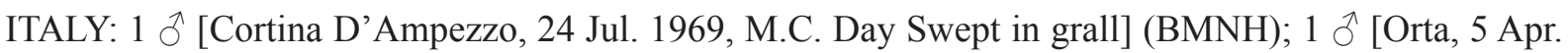
1950, G.J. Kerrich] (BMNH); 1 q [Dolomites, Seis am Schlein, 1-13 Jun. 1964] (BMNH); 3 우 [Abruzzo (AQ), L'Aquila, Aterno River, sweeping riparian veg. and Salix, 20 Jun. 1992, J. D. Pinto] (CNCI C-253).

JAPAN: $1 \widehat{\partial}$ [Hokkaido, Hidako Mts below Pyo Tan, 500 m, 14 Aug. 1996, L. Masner, S.S. J-12] (CNCI C-161); 1 ㅇ [Tsukuba, Ibaraki, 21 Jun. 1987, sweep, C.M. Yoshimoto] (CNCI C-182); 1 ㅇ [Hokkaido,

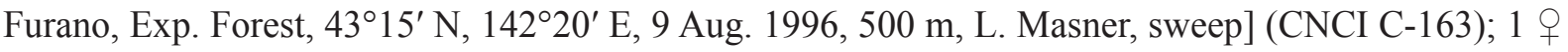
[Ibaraki, Tsukuba, Expo site, 21-31 May 1989, M.J. Sharkey] (CNCI C-214).

F.Y.R MACEDONIA: 1 q [Verge of oak wood above lake, Yugoslavia, Macedonia, Prespa Geril, 22 Jun. 1958, R.L. Coe.] (BMNH B.M. 1958-417).

MALTA: 3 ふふૈ, 1 q [May 1982, sp C.I.E. A. 13986, Alloxysta Först? sp. $q$ det. J. Quinlan, 1982] (BMNH); 1 §̊, 1 q [Malta, May 1982, sp. DI, C.I.E., A. 13986] (BMNH).

MOROCCO: 1 [ [Middle Atlas, flowers at edge of wood, Frane $5.500 \mathrm{ft}, 10 \mathrm{Jun}$. 1961, P.N. Lawrence] (BMNH B.M. 1961-328); 18 우 [Marrakech, Ouigane, $1000 \mathrm{~m}, 31^{\circ} 08^{\prime} \mathrm{N}, 8^{\circ} 05^{\prime} \mathrm{W}, 3-8$ May 1996, MT,

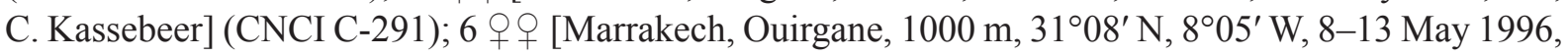
C. Kassebeer, MT] (CNCI C-295); 2 우 [Marrakech, Ouirgane, $1000 \mathrm{~m}, 31^{\circ} 08^{\prime} \mathrm{N}, 8^{\circ} 05^{\prime} \mathrm{W}, 4-11$ Oct. 1996, C. Kassebeer, MT] (CNCI C-279); 1 ㅇ [Marrakech, Ouirgane, 1000 m, 31 ${ }^{\circ} 08^{\prime}$ N, $8^{\circ} 05^{\prime} \mathrm{W}, 19-25$ Mar. 1997, C. Kassebeer, MT] (CNCI C-281); 1 [Marrakech, Ouirgane, 1000 m, 31 ${ }^{\circ} 08^{\prime}$ N, $8^{\circ} 05^{\prime}$ W, 11-17 Jun. 1996, C. Kassebeer, MT] (CNCI C-282); 1 ㅇ [Marrakech, Ouirgane, 1000 m, 31.08' N, 8.05' W, 17-23 Jun. 1996, C. Kassebeeer] (CNCI C-283).

NETHERLANDS: 1 ○ิ, 3 우 [Wageningen, 26 Jul. 1978, L. Huggert, swept in marsh] (CNCI C-310). 
POLAND: 1 ภึ, 2 우우 [Przemysl, 12 Sep. 1984, N.D. Springate] (BMNH).

PORTUGAL: 1 \& [Gondomar Douto Litoral, 18 Jun. 1962, NLH Krauss] (USNM).

ROMANIA: 1 [Brasov, $13 \mathrm{Km}$ S. of Brasov, vegetation under prunes, 11-12 Aug. 1967, B.H. and M.C., Cogan] (BMNH B.M. 1969-270).

RUSSIA: 1 [Russian Far East, Primorski Krai Lazovski Zapovednik, c.170 km E. of Vladivostok, Ta-

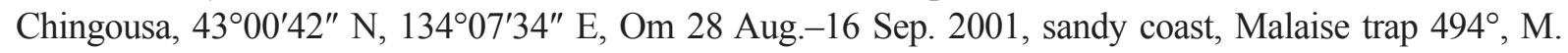
Quest coll., Alloxysta sp4, det. M. Forshage 2012] (BMNH B.M. 2009-59); 1 q [Primoskiy Kray Ussyriysk District, Gomotayozhnoye, 11-15 Aug. 2003, Malaise trap, M.V. Michailovskaya] (USNM).

SLOVAKIA: 1 ठૈ, 11 q [Slovakia, Čachtice environs, 2 Aug. 1991, L. Masner, sweep] (CNCI C-267).

SLOVENIA: 1 § [Postojne, 18 Jul. 1958, R.L. Coe, herbage in mixed forest] (BMNH B.M. 1958-417); 1 [ [Postojne, 18 Jul. 1958, R.L. Coe, wooded hill N.W. of town] (BMNH B.M. 1958-417); 1 ๙ [Slatna, Radovljica, 6 Aug. 1978, L. Huggert, swept on open wooded (deciduous) meadow] (CNCI C-275).

SWEDEN: 1 đ [Sk. Lund Zoological Mus. grounds Aug. 1976: 6 đ and 20 ๆ; Sö. Sandemar, T.H. and

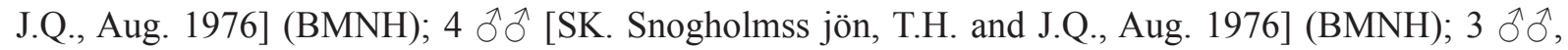
2 우 [Sk. Fyladalen, T.H. and J.Q., Aug. 1976] (BMNH); 6 우 [Sk., Rolsberga, Rovurekulan, RN1354/6188] (BMNH); 1 [Up. Solna Bergshamra, T.H. and J.Q., Aug. 1976] (BMNH); 1 q [Sö, Sandemars slott, T.H. and J.Q., Aug. 1976] (BMNH); 1 q [Up., Salna Bergshamra, T.H. and J.Q., Aug. 1976] (BMNH); 1 q [Sö, Sandemars slott, T.H. and J.Q., Aug. 1976] (BMNH); 1 q [Sö, Dalarö

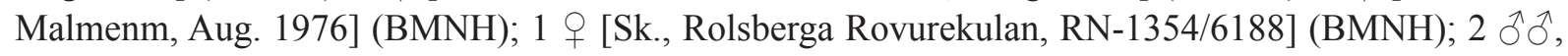
13 우 [Sk., Silvåkra Stensoffa, T.H. and J.Q., Aug. 1976, RN-1351/6176] (BMNH); 1 [So Malmen, Dalaro, J.Q. and T.H., 15-26 Aug. 1976] (BMNH); 1 đ [Sk., Snogholmssjön, T.H. and J.Q., Aug. 1976] (BMNH); 1 \& [Sö, Sandemar, T.H. and J.Q., Aug. 1976] (BMNH); 1 q [Sö, Dalarö Malmen, Aug. 1976]

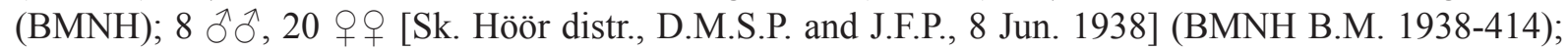
1 [Sk, S. Sandby Maryd, T.H. and J.Q., Aug. 1976] (BMNH); 1 \& [Sö, Gålö, Stegsholm, T.H. and J.Q., Aug. 1976] (BMNH); 5 우 [Sk., Lund Zoological Mus. grounds, Aug. 1976] (BMNH); 1 우 [Sk., Rolsberga Rovurekulan, T.H. and J.Q., Aug. 1976, RN-1354/6188] (BMNH); 1 क [So, Ludgo s:n, Tovetorp Field Station, 58.947750, 17.148542, sweep, around station rounds, M. Gates and M. Buffington, 6 Aug. 2012] (USNM).

SWITZERLAND: 201 $\widehat{\partial}, 85$ 우 [Dielsdorf, 650 m, 17 Aug. 1984, L. Masner, sweep] (CNCI C-290); 1 ㅇ [Solothurn, Weissenstein, $1225 \mathrm{~m}, 4^{\circ} 15^{\prime} 10^{\prime \prime}$ N, 7³0'00" E, 17 Jun. 1999, Goulet/White, lush meadow] (CNCI C-285); 3 우 [Turgau, Unterwasser environs, 1440 m, 4 Aug. 1984, L. Masner, sweeping] (CNCI C-293).

UNITED KINGDOM: England: 4 + $\odot$ [Lincolnshire, Moorside near Fulsby Wood, 22-25 Aug. 1986,

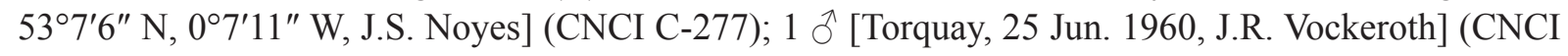
C-299).

\section{Distribution}

Cosmopolitan.

Certain records: Andorra (Ferrer-Suay et al. 2011: 359), Corsica (Ferrer-Suay et al. 20131), England (Westwood 1833: 495; Müller et al. 1999: 346; van Veen et al. 2003: 450), Japan (Ferrer-Suay et al. 2013i), Madeira (Borges et al. 2008; Ferrer-Suay et al. 2012g: 13), Mexico (Ferrer-Suay et al. 2013m: 
37), New Zealand (Valentine 1975: 59; Ferrer-Suay et al. 2012h: 237), Serbia and Montenegro (FerrerSuay et al. 2013b: 363).

Uncertain records: Australia (Girault 1932: 3), Austria (Giraud 1860: 127; Hellén 1963: 16), Belgium (Fabianus 1900), Brazil (Peronti et al. 2007: 132), Canada (Fitch 1861: 841), Chile (Guerra et al. 1998: 335), England (Curtis 1838: 688; Cameron 1883: 366; Dalla Torre \& Kieffer 1910: 285, 288; West et al. 1998: 1458), Finland (Hellén 1963: 6), France (Kieffer 1902a: 15-16; 1902b: 70, 600; De Gaulle 1908: 26), Germany (Hartig 1840: 199; Hübner et al. 2002: 507; Höller et al. 1993: 13), Greenland (Buhl 1997: 164), Hungary (Dalla Torre \& Kieffer 1910: 285; Fülöp et al. 2010: 55), Ireland (O’Connor \& Nash 1997), Israel (Argaman 1988: 114), Italy (Pagliano 1995: 3), Lappland (Zetterstedt 1838: 410; Hellén 1963: 16), Norway (Hellén 1966: 393; Westrum et al. 2010), Poland (Kierych 1979b: 15; Krawczyk et al. 2009: 161), Romania (Ionescu 1969: 261; Feraru \& Mustata 2005: 75), Russia (Belizin 1962: 127; Hellén 1963: 16; Bokina 1997: 435), Scotland (Cameron 1883: 366), Spain (Torras-Casals 1996: 196), Sweden (Thomson 1862: 406; 1877: 814), The Netherlands (Hellén 1963: 16; Andrews 1978: 92), USA (Fitch 1861: 841; Kieffer 1909: 481; Sullivan \& van den Bosch 1971; Mertins 1985: 186).

New records: Cyprus, Czech Republic, Denmark, Iran, Malta, Morocco, Portugal, Romania, The Netherlands and Switzerland.

\section{Alloxysta xanthocera (Thomson, 1862)}

Figs 3.19, 5.22

Allotria xanthocera Thomson, 1862: 407. Type: deposited in MZLU (examined).

Allotria (Allotria) xanthocera - Dalla Torre \& Kieffer 1902: 41.

Charips (Charips) xanthocerus - Dalla Torre \& Kieffer 1910: 282.

Alloxysta xanthocera - Hellén 1963: 18.

\section{Diagnosis}

Alloxysta xanthocera is characterized by a closed radial cell that is 2.6 times as long as wide (Fig. 5.22); the presence of pronotal carinae; propodeal carinae that form a wide plate with curved sides; and antenna with rhinaria and club shape not beginning on the same flagellomere, club shape beginning on F2, rhinaria beginning on F1, F1 longer than pedicel and F2, and F2-F4 subequal in length (Fig. 3.18). No other known species of Alloxysta is closely similar to A. xanthocera.

\section{Material examined}

\section{Lectotype}

SWEDEN: $q$ [xanthocera (handwritten)], [xanthocera (handwritten)], [Lectotype Allotria xanthocera Thomson, 1862 q desig. M. Ferrer-Suay 2011 (red label)], [Alloxysta xanthocera (Thomson, 1862) $q$ M. Ferrer-Suay det. 2011], [ZML. 2011066 (green label)] (MZLU).

\section{Distribution}

Palaearctic.

Certain records: Sweden (Thomson 1862: 407).

Uncertain records: Finland (Hellén 1963: 18), Israel (Argaman 1988: 115), Romania (Ionescu 1969: 262). 
Alloxysta xanthopa (Thomson, 1862)

Figs 3.19, 5.23

Allotria xanthopa Thomson, 1862: 408. Type: deposited in MZLU (examined).

Dilyta xanthopa - Kieffer 1900: 114.

Alloxysta (Alloxysta) xanthopa - Dalla Torre \& Kieffer 1902: 39.

Alloxysta xanthopa - Andrews 1978: 93.

\section{Diagnosis}

Alloxysta xanthopa is characterized by a completely open radial cell that is 2.4 times as long as wide (Fig. 5.23); the presence of pronotal carinae; propodeal carinae that form a plate; and antenna with rhinaria beginning on F3, F1 longer than pedicel and slightly longer than F2, F2 shorter than F3, and F3 subequal to F4 (Fig. 3.19). These features easily differentiate it from other species of Alloxysta.

\section{Material examined}

\section{Lectotype}

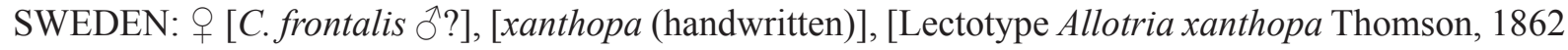
$\uparrow$ desig. M. Ferrer-Suay 2011 (red label)], [Alloxysta xanthopa (Thomson, 1862) + M. Ferrer-Suay det. 2011], [ZML. 2011068 (green label)] (MZLU).

\section{Additional specimens ( 1 \%)}

JAPAN: 1 q [Mt. Hyachine, Iwate, 500 m, 21 Jun. 1989, sweep, M.J. Sharkey] (CNCI C-201).

\section{Distribution}

Palaearctic.

Certain records: Sweden (Thomson 1862: 409), Nepal (Ferrer-Suay et al. 2013i).

New record: Japan.

Apocharips trapezoidea (Hartig, 1841)

Figs 3.20, 5.24

Xystus trapezoideus Hartig, 1841: 352. Type: deposited in ZSM (examined).

Allotria trapezoideus - Taschenberg 1866: 130.

Allotria trapezoidea - Cameron 1884: 267.

Dilyta trapezoidea - Kieffer 1900: 114. — Evenhuis 1982: 26.

Alloxysta (Alloxysta) trapezoidea - Dalla Torre \& Kieffer 1902: 39.

Apocharips trapezoidea - Menke \& Evenhuis 1991: 152.

\section{Diagnosis}

Apocharips trapezoidea is mainly characterized by a completely open radial cell that is 1.0 times as long as wide (Fig. 5.24); the presence of pronotal and propodeal carinae; an apex of scutellum with M-shaped carinae; female antenna with rhinaria beginning on F5, F1 longer than pedicel and F2, F2 subequal to F3, and F3 shorter than F4 (Fig. 3.20); male antenna with rhinaria beginning on F1, F1 longer than pedicel and F2, and F2-F4 subequal in length. This species is easily differentiated from other species of Apocharips by the completely open radial cell with parallel R1 and Rs veins (Fig. 5.24). The closest 
congener is $A$. hansoni Menke, 1993, but it can be easily differentiated by the presence of radial carinae around the clypeus (absent in A. trapezoidea but present in A. hansoni).

\section{Material examined}

\section{Lectotype}

GERMANY: $\hat{\jmath}$, [lectotype H.H. Evenhuis (orange label)], [Xystus trapezoideus Hartig det. H.H. Evenhuis 1980], [Apocharips trapezoidea §̊ (Hartig, 1840) det. JP-V 2007] (ZSM).

Additional specimens $(4 \hat{\partial} \widehat{\partial}, 5$ $+q)$

FRANCE: 2 $ぇ, 1$ [ [Var: St. Tropez, 12 Jun. 80, Boucek] (BMNH); 1 [Var: St. Tropez, 16 Jun. 80, Boucek] (BMNH).

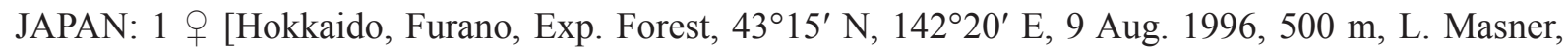
sweep] (CNCI C-163).

SPAIN: 1 [Calella, A. Costa NE, Jun. 1971, Boucek] (BMNH); 1 [Canary Is., Tenerife, El Medano, 18 Mar. 1983, K.J. Hedqvist, Hedqvist coll.] (BMNH B.M. 2011-27).

SWEDEN: 1 \& [Sö, Sandemars slott, T.H. and J.Q., Aug. 1976, Apocharips xanthocephala det. N.D.M. Fergusson, 1985] (BMNH).

TUNISIA: 1 ô [Tunis 1979, Alloxysta †? sp. det. J. Quinlan, 1979] (BMNH).

\section{Distribution}

Palaearctic.

Certain records: Andorra (Pujade-Villar 2005: 545), Corsica (Ferrer-Suay et al. 20131), France (FerrerSuay et al. 2015a), Germany (Hartig 1841: 352), Canary Islands (Pujade-Villar 2005: 545), Peninsular Spain (Nieves-Aldrey et al. 2003: 39), Tunisia.

Uncertain records: Austria (Andrews 1978: 91), Bulgaria and Balkan Peninsula (Vasileva-Sumnalieva 1976: 23), England (Andrews 1978: 91), Ethiopia (Silvestri 1915: 273), Finland (Hellén 1963: 5), Ireland (O’Connor \& Nash 1997), Italy (Silvestri 1915: 274), Scotland (Andrews 1978: 91), Sweden (Thomson 1862: 409).

Dilyta aleevae Pujade-Villar \& Paretas-Martínez, 2011

Fig. 6.1

Dilyta aleevae Paretas-Martínez et al., 2011a: 31. Type: deposited in ZIN (examined).

\section{Diagnosis}

Dilyta aleevae is mainly characterized by a lack of punctures on the posterior part of the metasoma; female antenna with F1 slightly shorter than pedicel, F1 longer than F2, F2 slightly longer than F3 and F4, F3 subequal to F4, a slight clavation from F5, and sensilla beginning on F6 (Fig. 6.1); male antenna with F1 longer than pedicel and slightly arched, F1 longer than F2 and F3, F2 subequal to F3, F3 shorter than F4, F1 as long as F4, a slight clavation from F4, and sensilla beginning on F6. It is similar to D. rathmanae Menke \& Evenhuis, 1991 but can be differentiated by the proportions of the flagellomere (F1 is longer than F2, F2 is slightly longer than F3 or F4, and F3 is subequal to F4 in D. alevae females (Fig. 6.1), while F1 is almost double the length of F2, F3 or F4, and F2 or F3 is subequal to F4 in 
D. rathmanae females (Fig. 6.4); and F1 is longer than the pedicel in D. alevae males, but subequal to the pedicel in D. rathmanae males).

\section{Material examined}

\section{Holotype}

KAZAKHSTAN: + [Shchuchinsk, 15.IX, Kokchetavskaya oblast, M.Alejeva 1951 (in Russian)], [Holotypus Dilyta aleevae m V. Belizin det. (red)], [Dilya sp. aff talitzkii Kovalev O. det.1985, Holotype Dilyta aleevae sp. n. Pujade-Villar and Paretas-Martínez (red)] (ZIN).

\section{Paratypes}

KAZAKHSTAN: 2 q $q, 2 \widehat{\partial}$, same locality data as holotype, [Paratypus Dilyta aleevae m V. Belizin det. (red), Paratype Dilyta aleevae sp. n. Pujade-Villar and Paretas-Martínez (red)] (ZIN).

\section{Distribution}

Palaearctic.

Certain records: Kazakhstan (Paretas-Martínez et al. 2011a: 32).

\section{Dilyta japonica Paretas-Martínez \& Ferrer-Suay, 2011}

Fig. 6.2

Dilyta japonica Paretas-Martínez \& Ferrer-Suay in Paretas-Martínez et al., 2011a: 32. Type: deposited in CNCI (examined).

\section{Diagnosis}

Dilyta japonica is mainly characterized by a punctate area on the posterior part of the metasoma and the female antenna slightly clavate from F6 with F1 very long and thin, much longer than the pedicel (almost double); F1 longer than F2, F3, F4, and F5; and F7-F11 wider than the previous segments (Fig. 6.2). According to these features, there is no other species of Dilyta very similar to D. japonica.

\section{Material examined}

\section{Holotype}

JAPAN: + [Hokkaido, Horota 800 m, 5 Jul. 1989, Sweep, M.J. Sharkey; Holotype Dilyta japonica sp. n. Paretas-Martínez \& Ferrer-Suay (red)] (CNCI C-449).

\section{Distribution}

Certain records: Japan (Paretas-Martínez et al. 2011a: 32).

Dilyta longinqua Paretas-Martínez \& Pujade-Villar, 2011

Fig. 6.3

Dilyta longinqua Paretas-Martínez \& Pujade-Villar in Paretas-Martínez et al., 2011a: 32. Type: deposited in ZIN (examined).

\section{Diagnosis}

Dilyta longinqua is mainly characterized by a punctate area on the posterior part of the metasoma; female antenna slightly clavate from F6 with F1 subequal to pedicel or slightly longer, F2 shorter than 
F3, F3 shorter than F4, and sensilla beginning on F6 (Fig. 6.3); male antenna slightly clavate from F3 with $F 1$ subequal to pedicel, $F 2$ shorter than $F 1$ or F3, F3 subequal to F1, F3-F12 wider than the previous flagellomeres, and sensilla beginning on F3. It is similar to D. subclavata, but can be differentiated by the proportions of the flagellomere (F1 is slightly shorter than or subequal to pedicel, F2 is subequal to F3, and F4 is slightly shorter than F1, but longer than F2 or F3 in D. subclavata females (Fig. 6.6), while F1 is slightly longer than pedicel, F2 and F3 are each shorter than F1, F1 is subequal to F4, F4-F12 are wider than previous flagellomeres, antenna are slightly clavate from F4, and sensilla begin on F4 in males of D. subclavata).

\section{Material examined}

\section{Holotype}

CHINA: + [Shaowu, Fukien, 10 Nov. 1945, M.Chao; from Psyllidae on firmian (in Russian)], [Holotypus Dilyta longinqua V. Belizin det. (red)], [Holotype Dilyta longinqua sp. n. Paretas-Martínez and PujadeVillar (red)] (ZIN).

\section{Paratypes}

CHINA: $2 \hat{\jmath}, 2$ 우, same locality data as for holotype, [Paratypus Dilyta aleevae m V. Belizin det. (red)], [Paratype Dilyta longinqua sp. n. Paretas-Martínez and Pujade-Villar (red)] (ZIN).

\section{Distribution}

Palaearctic.

Certain records: China (Paretas-Martínez et al. 2011a: 34).

Dilyta sinica Ferrer-Suay \& Paretas-Martínez, 2011

Fig. 6.5

Dilyta sinica Ferrer-Suay \& Paretas-Martínez in Paretas-Martínez et al., 2011a: 35. Type: deposited in UB (examined).

\section{Diagnosis}

Dilyta sinica is mainly characterized by punctures on the posterior half of the metasoma and the male antenna slightly clavate from F4 with F1 very long, wide, and arched, F1 much longer than pedicel (almost double), F1 longer than F2 and F3 together, F2 slightly shorter than or subequal to F3, F4 longer than F2 and F3, F4-F12 wider than previous segments, and sensilla beginning on F4 (Fig. 6.5). According to these features, there is no other species of Dilyta very similar to D. sinica.

\section{Type material}

\section{Holotype}

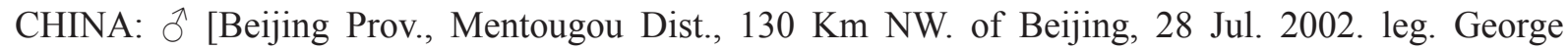
Melika.], [Liyan Ling (Linshan mnt.), $1749 \mathrm{~m}$ a.s.1., $40^{\circ} 00.279^{\prime} \mathrm{N}, 115^{\circ} 30.758^{\prime} \mathrm{E}$, sweep, on alpine mead], [Holotype Dilyta sinica sp. n. Ferrer-Suay and Paretas-Martínez (red)] (UB).

\section{Paratype}

CHINA: $1 \delta^{\Uparrow}$ [Beijing, $130 \mathrm{Km} \mathrm{N}$ of Liyan Ling (Linshan moun., leg. H. Baur, 40 $00.279^{\prime} \mathrm{N}$, $115^{\circ} 30.758^{\prime}$ E, sweeping, 4 Aug. 2002], [Paratype Dilyta sinica sp. n. Ferrer-Suay and Paretas-Martínez (red)] (SPL). 


\section{Distribution}

Palaearctic.

Certain records: China (Paretas-Martínez et al. 2011a: 35).

\section{Dilyta subclavata Förster, 1869}

Fig. 6.6

Dilyta subclavata Förster, 1869: 338. Type: deposited in NMW (examined).

Alloxysta (Alloxysta) subclavata - Dalla Torre \& Kieffer 1910: 255.

Dilyta subclavata - Hellén 1963: 5.

\section{Diagnosis}

Dilyta subclavata is mainly characterized by a punctate area on the posterior part of the metasoma; female antenna slightly clavate from F6 with F1 slightly shorter than or subequal to pedicel, F2 subequal to F3, F4 slightly shorter than F1 but longer than F2 and F3, and sensilla beginning on F6-F7 (Fig. 6.6); male antenna slightly clavate from F4 with F1 slightly longer than pedicel and sometimes slightly arched, F2 shorter than F1 and F3, F3 shorter than F1, F1 subequal to F4, F4-F12 wider than previous segments, and sensilla beginning on F4. It is similar to D. longinqua, but can be differentiated by the proportions of the flagellomere (F1 is subequal to pedicel or slightly longer, F2 is shorter than F3, F3 is shorter than F4, antenna are slightly clavate from F6, and sensilla begin on F6 in D. longinqua females (Fig. 6.3), while F1 is subequal to pedicel, F2 is shorter than F1 or F3, F3 is subequal to F1, F3-F12 are wider than the previous flagellomeres, antenna are slightly clavate from F3, and sensilla begin on F3 in males of D. longinqua).

\section{Material examined}

\section{Lectotype}

GERMANY: 2 + , on one pin [Först.; Collect. G Mayr], [Dil. subclavata Förster, Type; Dilyta subclavata Frst.], [Dilyta subclavata Förster 2 우 det. H.H. Evenhuis 1976 (orange)], [das rechte Weibchen wäre als Lektotypus zu wählen H.H. Evenhuis (orange)] (NMW).

\section{Additional specimens $(5 \precsim \widehat{\partial}, 25$ 우 )}

AUSTRIA: $1 \widehat{\delta}$ [Ex Gelechia musco sel Z, Merkestein, 29 Jun. 1959, C Jan. L.B. Coll. No. 43.64, Alloxysta (Först) Hellén? sp. det. J. Quinlan, 1965] (BMNH) (handwritten).

FRANCE: 1 ô, 1 q [Avignon (Vancluse), 13 May 1981, Ex Psylla pyri on pear trees, Collector: F. Herard, European Parasite Lab. V.S.D.A. Sèvres, Dilyta subclavata + Forst. det J. Quinlan, 1982] (BMNH).

GERMANY: $1 \hat{\sigma}$ [Schwarzwald, Todtmoos environs, 6 Aug. 1984, L. Masner, screen sweeping] (CNCI C-235).

JAPAN: 1 q [Hokkaido, 20 km N. Akkeshi, marsh, 100 m, 15 Jul. 1996, L. Masner, sweep] (CNCI C-160).

NETHERLANDS: 1 ô, 1 [ [Langbrock (prov. Wfrecht), 10 Jun. 1976, collected from leaf of Fraxinus excelsis leg H.H. Evenhuis, near colony of Psyllopsis fraxini (Hom., Psyllidae) (handwritten), ô, Dilyta subclavata Förster det. H.H. Evenhuis 1976] (BMNH).

NORWAY: 1 đ̊, 1 \& [Hoy Fauna Lonningen, 15 Aug. 1980, F. Jelosa] (BMNH); 1 [ [Kronli, Falkflanf, Nsy: Bodø 14 Jul. 1980, A. Fjeldsa leg] (BMNH). 
POLAND: 4 우 [Borowki, N.W. Poland, 16-28 Sep. 1934, G.J. Kerrich] (BMNH).

RUSSIA: 14 우 [Russian Far East, Primorskii Krai Lazovski Zapovednik, c. 170 Km E, Vladivostok, Lazo, 4330'33" N, 13406'59" E, 1375 m, 2 Jun.-3 Jul. 2001, mountain top, Malaise trap 458, M. Quest coll., Dilyta subclavata det. M. Forshage 2012] (BMNH B.M. 2009-59).

SPAIN: 1 + [Madrid, Vaciamadrid, 19 Oct. 1978, J.S. Noyes] (BMNH B.M. 1978-488).

SWEDEN: 1 q [Sö, Sandemars slott, T.H. and J.Q., Aug. 1976] (BMNH).

\section{Distribution}

Holarctic.

Certain records: Germany (Förster 1869: 338), Hungary (Fülöp et al. 2010: 54), Norway (ParetasMartínez et al. 2011a: 36), Peninsular Spain (Nieves-Aldrey et al. 2003: 39), The Netherlands (ParetasMartínez et al. 2011a: 36), USA (Paretas-Martínez et al. 2011a: 36).

Uncertain records: England (Marshall 1870: 181; Fergusson 1986: 16), Finland (Hellén 1963: 5), France (De Gaulle 1908: 26; Dalla Torre \& Kieffer 1910: 255), Ireland (Marshall 1870: 181), Moldova (Belizin 1966: 7), Poland (Kierych 1979a: 458), Romania (Feraru \& Mustata 2005: 75), Sweden (Thomson 1877: 814; Hellén 1963: 5).

New records: Austria, Japan, Norway and Russia.

Phaenoglyphis abbreviata (Thomson, 1877)

Figs 6.7, 7.1

Allotria (Auloxysta) abbreviata Thomson, 1877: 812. Type: deposited in MZLU (examined).

Allotria abbreviata - Dalla Torre 1893: 29.

Hemicrisis abbreviata - Kieffer 1900: 113.

Phaenoglyphis abbreviata - Dalla Torre \& Kieffer 1902: 42. — Andrews 1978: 93.

Phaenoglyphis (Phaenoglyphis) abbreviata - Hellén 1963: 7.

\section{Diagnosis}

Phaenoglyphis abbreviata is mainly characterized by a closed radial cell that is 2.5 times as long as wide; the presence of pronotal and propodeal carinae; the presence of notauli; rounded scutellar foveae separated by a small and well-defined carina (Fig. 7.1); and female antenna with rhinaria beginning on F3, F1 subequal to pedicel, F1 longer than F2, F2 shorter than F3, and F3 subequal to F4 (Fig. 6.7). It is similar to $P$. moldavica, but can be differentiated by the proportions of the flagellomere (F1 is longer than F2 and F2 is shorter than F3 in P. abbreviata (Fig. 6.7), while F1-F3 are subequal in length in P. moldavica (Fig. 6.14)), and the shape of the scutellar foveae (small, rounded, and separated by a wide carina in P. abbreviata (Fig. 7.1) but big and separated by a fine carina in P. moldavica (Fig. 7.8)).

\section{Material examined}

\section{Lectotype}

SWEDEN: 9 [Esperöd 12 Aug 38 (handwritten)], [O], [X. nitidus (handwritten); 1972, 50], [lectotype H.H. Evenhuis (orange label)], [Allotria (Auloxysta) abbreviata Thomson, det. H.H. Evenhuis 1977], [ZML. 2004, 346 (green label)] (MZLU). 
Paralectotype

SWEDEN: $q$ [abbreviate (handwritten)], [Sm.; 1969, 89 (green label)], [1972, 49], [paralectotype H.H. Evenhuis (orange label)], [Allotria (Auloxysta) abbreviata Thomson, det. H.H. Evenhuis 1977], [ZML. 2004, 345] (MZLU).

\section{Distribution}

Palaearctic.

Certain records: France (Ferrer-Suay et al. 2015a), Sweden (Thomson 1877: 812).

Uncertain records: Finland (Hellén 1963: 7), Romania (Ionescu 1969: 273).

Phaenoglyphis evenhuisi Pujade-Villar \& Paretas-Martínez, 2006

Figs $6.8,7.2,10.1$

Phaenoglyphis evenhuisi Pujade-Villar \& Paretas-Martínez, 2006: 479. Type: deposited in UB (examined).

\section{Diagnosis}

Phaenoglyphis evenhuisi is mainly characterized by having the pronotum and mesoscutum completely sculptured; a closed radial cell that is 3.0 times as long as wide; notauli are only indicated; scutellar foveae with a transverse posterior carina that is completely defined and separated by a carina (Fig. 7.2); female antenna with rhinaria and club shape beginning on F4, F1 longer than pedicel and F2, F2 subequal to F3, and F3 shorter than F4 (Fig. 6.8). It is similar to P. pubicollis, but can be differentiated by where the rhinaria begin (F4 in P. evenhuisi (Fig. 6.8) but F1 in P. pubicollis (Fig. 6.17)) and the shape of the notauli (only indicated in P. evenhuisi (Fig. 7.2) while only indicated anteriorly and well-marked posteriorly in P. pubicollis (Fig. 7.11)).

\section{Material examined}

\section{Holotype}

ANDORRA: $q$ [Santa Coloma (AND), 16-30 Jun. 1993, Trampa Malaise, J. Pujade leg (white label)], [Phaenoglyphis nr. nigripes (Thomson), det. H.H. Evenhuis 1997 (white label)], [Holotype (red label)], [Phaenoglyphis evenhuisi J. P-V and Paretas-Martínez sp. nov., female (white label)] (UB).

\section{Distribution}

Palaearctic.

Certain records: Andorra (Pujade-Villar \& Paretas-Martínez 2006: 479), France (Ferrer-Suay et al. 2015a).

Phaenoglyphis fuscicornis (Thomson, 1877)

Figs 6.9, 7.3

Allotria (Auloxysta) fuscicornis Thomson, 1877: 813. Type: deposited in MZLU (examined).

Phaenoglyphis fuscicornis - Kieffer 1900: 113. — Andrews 1978: 94.

Phaenoglyphis (Phaenoglyphis) fuscicornis - Hellén 1963: 6. 


\section{Diagnosis}

Phaenoglyphis fuscicornis is mainly characterized by a closed radial cell that is 2.8 times as long as wide in both males and females; the presence of pronotal and propodeal carinae; the presence of notauli; scutellum with two oval scutellar foveae separated by a carina and not delimited posteriorly (Fig. 7.3); female antenna with club shape beginning on F3, rhinaria beginning on F1, F1 longer than pedicel, F1 slightly longer than F2, F2 slightly shorter than F3, and F3 subequal to F4 (Fig. 6.9); male antenna with club shape beginning on F3, rhinaria beginning on F5, F1 curved, F1 longer than pedicel and F2, F2 subequal to F3, and F3 slightly shorter than F4. It is similar to P. americana Baker, 1896 (Figs 8.3, 9.3), but can be differentiated by where the club shape begins in females (on F3 in P. fuscicornis (Fig. 6.9), but on F2 in $P$. americana), the shape of the notauli (deeply excavated anteriorly and weakly posteriorly in P. fuscicornis (Fig. 7.3), but clearly visible all along in P. americana) and where rhinaria and club shape begin in males (club shape begins in F3 and rhinaria in F5 in P. fuscicornis, while both begin in F2 in P. americana).

\section{Material examined}

\section{Lectotype}

SWEDEN: ${ }^{\lambda}$, [Lund], [1969, 91 (green label)], [1972, 52], [ZML.2004, 342 (green label)], [Lectotype

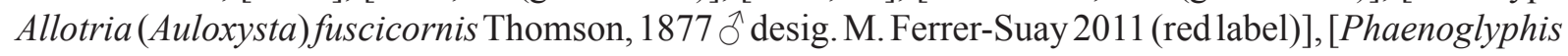
fuscicornis (Thomson, 1877) § M. Ferrer-Suay det. 2011] (MZLU).

\section{Paralectotype}

SWEDEN: 1 ô [L-d], [1972, 53], [ZML.2004, 341 (green label)], [Paralectotype Allotria (Auloxysta)

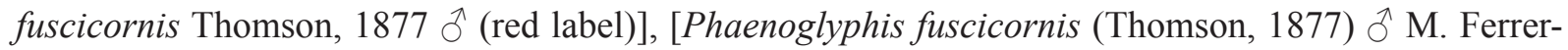
Suay det. 2011] (MZLU).

\section{Distribution}

Palaearctic.

Certain records: France (Ferrer-Suay et al. 2015a: 132), Sweden (Thomson 1877: 813).

Uncertain records: Finland (Hellén 1963: 7), Romania (Ionescu 1969: 275).

Phaenoglyphis heterocera (Hartig, 1841)

Figs 6.10, 7.4

Xystus heterocerus Hartig, 1841: 351. Type: deposited in ZSM (examined).

Allotria heterocerus - Taschenberg 1866: 129.

Allotria heterocera-Cameron 1890: 234.

Dilyta heterocera - Kieffer 1900: 114.

Allotria (Allotria) heterocera - Dalla Torre \& Kieffer 1902: 40.

Charips (Charips) heterocerus - Dalla Torre \& Kieffer 1910: 275.

Phaenoglyphis heterocera - Evenhuis 1982: 24.

\section{Diagnosis}

Phaenoglyphis heterocera is mainly characterized by rounded scutellar foveae not delimited (Fig. 7.4) and antenna with F1 shorter than pedicel in females (Fig. 6.10), but longer in males, and rhinaria and club shape beginning on F3. It is similar to P. stenos Andrews, 1978, but can be differentiated by where the rhinaria begin (on F3 in P. heterocera, but F2 in P. stenos) and flagellomere proportions (F1 is shorter 
than pedicel and F1-F3 are subequal in length in P. heterocera females (Fig. 6.10), but F1 is longer than pedicel and F1-F3 are not subequal in $P$. stenos females).

\section{Material examined}

\section{Lectotype}

GERMANY: $\widehat{\partial}[637],[ð]$, [lectotype H.H. Evenhuis (orange label)], [Xystus heterocerus Hartig, det. H.H. Evenhuis 1980] (ZSM).

\section{Paralectotype}

GERMANY: $1 \precsim$ [In collection Hartig as Xystus heterocerus; Paralectotype Xystus heterocerus (Hartig, 1841) $\widehat{\jmath}$ (red label)], [Phaenoglyphis heterocera (Hartig, 1841) § M. Ferrer-Suay det. 2011] (ZSM).

Additional specimens $(5 \hat{\partial} \widehat{\partial}, 3$ + $\odot)$

AUSTRIA: $2 \lesssim \widehat{\jmath}$ [Leibnitz, R. Mur, 3 Jun. 1969, B.H. and M.C. Cogan, R.I. and R. Vane-Wright] (BMNH B.M. 1970-152).

EGYPT: 1 ठ [Siwa, 18-19 Apr. 1935, J. Ower-Cooper, Armstrong College Expedition, gardens] (BMNH B.M. 1935-354).

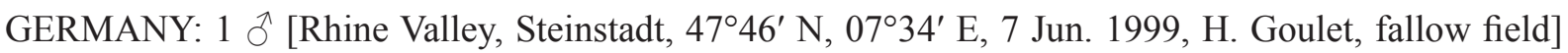
(CNCI C-232).

HUNGARY: 1 ठૈ [Baranya Beremend, 24 Apr.-9 May 1963, L. Horacsek] (BMNH B.M. 1964-373).

JAPAN: 1 앙k [Hoido, Aizan, 800 m, 4 Jul. 1989, M.J. Sharkey, sweep] (CNCI C-186); 2 우 [Hokkaido, Sapporo, Wisumai, 100 m, 30 Jun. 1989, M.J. Sharkey, sweep] (CNCI C-194).

\section{Distribution}

Palaearctic.

Certain records: Germany (Hartig 1841: 351).

Uncertain records: Austria (Dalla Torre \& Kieffer 1910: 275), England (Cameron 1890: 234), France (Kieffer 1902b: 598; De Gaulle 1908: 26), Romania (Feraru \& Mustata 2005: 75), Sweden (Andrews 1978: 84), The Netherlands (Dettmer 1925: 124).

New records: Egypt, Hungary and Japan.

\section{Phaenoglyphis insperatus Belizin, 1973}

Figs $6.11,7.5$

Phaenoglyphis insperatus Belizin, 1973: 36. Type: deposited in ZIN (examined).

\section{Diagnosis}

Phaenoglyphis insperatus is mainly characterized by oval scutellar foveae separated by a carina and slightly open posteriorly (Fig. 6.11); female antenna with rhinaria and club shape beginning on the last three quarters of F1, F1 longer than pedicel and F2, and F2-F4 subequal in length (Fig. 7.5). It is similar to $P$. stricta, but can be differentiated by the shape of scutellar foveae (they are rounded and slightly open posteriorly in P. insperatus (Fig. 7.5), while they have straight sides and are open both anteriorly 
and posteriorly in P. stricta (Fig. 7.14)) and the size of the radial cell (2.9 times as long as wide in P. insperatus but 2.4 times in P. stricta).

\section{Material examined}

\section{Holotype}

TAJIKISTAN: + [Kukhilal 2400, r. Pyandzh, V. Tobias, 18 Jul. 65] (ZIN).

\section{Distribution}

Palaearctic.

Certain records: Tajikistan (Belizin 1973: 36).

Phaenoglyphis insularis (Belizin, 1973)

Figs 6.12, 7.6

Auloxysta insularis Belizin, 1973: 37. Type: deposited in ZIN (examined).

Phaenoglyphis insularis - Pujade-Villar et al. 2010a: 287.

\section{Diagnosis}

Phaenoglyphis insularis is mainly characterized by a lack of notauli; rounded scutellar foveae separated by a weak carina and open at the bottom (Fig. 7.6); and antenna with rhinaria and club shape beginning on F3, F1 longer than pedicel and slightly longer than F2, F2 slightly longer than F3, and F3 subequal to F4 (Fig. 6.12). It is similar to P. calverti, but can be differentiated by the proportion of the F2-F3 (F2 is longer than F3 in P. insularis (Fig. 6.12), but shorter than F3 in P. calverti) and the shape of the mesoscutum (very gibbous with very few setae in P. insularis (Fig. 7.6), but not gibbous with a few setae only present on the anterior margin in $P$. calverti).

\section{Material examined}

\section{Holotype}

RUSSIA: + [Severo-Kuril'sko-v, Paramushir, tli na ive, 5 Aug. 1964; Holotypus Auloxysta insularis V. Belizin det. $\circ$ (red label)] (ZIN).

\section{Distribution}

Palaearctic.

Certain records: Russia (Belizin 1973: 37).

\section{Phaenoglyphis longicornis (Hartig, 1840)}

Figs $6.13,7.7$

Xystus longicornis Hartig, 1840: 199. Type: deposited in ZSM (examined).

Allotria longicornis - Taschenberg 1866: 129.

Allotria (Allotria) longicornis - Dalla Torre \& Kieffer 1902: 40.

Charips (Charips) longicornis - Dalla Torre \& Kieffer 1910: 277.

Alloxysta longicornis - Andrews 1978: 84.

Phaenoglyphis longicornis - Evenhuis 1978: 172. 


\section{Diagnosis}

Phaenoglyphis longicornis is mainly characterized by a closed radial cell that is 2.7 times as long as wide; the presence of pronotal and propodeal carinae; the presence of notauli; oval scutellar foveae with straight margins separated by a carina and not delimited at the bottom (Fig. 7.7); female antenna with rhinaria beginning on F1, F1 longer than pedicel and F2, F2 subequal to F3, and F3 shorter than F4 (Fig. 6.13). It is similar to P. stricta, but they can be differentiated by where rhinaria begin (on all of the surface of F1 in P. longicornis (Fig. 6.13), while only on the distal three quarters of F1 in P. stricta (Fig. 6.19)), the shape of the scutellar foveae (not delimited on the bottom in P. longicornis (Fig. 7.7), while not delimited on top and bottom in P. stricta (Fig. 7.14)), and the size of the radial cell (2.7 times as long as wide in P. longicornis but 2.4 times in P. stricta).

\section{Material examined}

\section{Holotype}

GERMANY: $q$ [711], [†], [holotype (red label)], [Xystus longicornis Hartig, det. H.H. Evenhuis 1977] (ZSM).

\section{Distribution}

Palaearctic.

Certain records: France (Ferrer-Suay et al. 2015a), Germany (Hartig 1840: 199), India (Ferrer-Suay et al. 2013i).

Uncertain records: England (Dalla Torre \& Kieffer 1910: 277), Romania (Ionescu 1969: 252), Scotland (Cameron 1886: 86), Sweden (Thomson 1862: 407).

Phaenoglyphis moldavica Ionescu, 1969

Figs $6.14,7.8$

Phaenoglyphis moldavica Ionescu, 1969: 275. Type: deposited in MGAB (examined).

\section{Diagnosis}

Phaenoglyphis moldavica is mainly characterized by well-marked notauli with a line of setae at each side; rounded scutellar foveae separated by a carina and completely defined (Fig. 7.8); antenna with rhinaria and club shape beginning on F3 in both males and females; female antenna with pedicel-F4 subequal in length (Fig. 6.14); male antenna with F1 subequal to pedicel and longer than F2, F2 shorter than F3, and F3 subequal to F4. According to these features, there is no other Phaenoglyphis species similar to $P$. moldavica.

\section{Material examined}

Holotype

ROMANIA: $q$ [Phaenoglyphis moldavica $\mathrm{n}$. sp (red label)] (MGAB).

\section{Paratype}

ROMANIA: đ̊ [Phaenoglyphis moldavica $\mathrm{n}$. sp (handwritten); ciric-iasi 1 Jul. 1957. § $\uparrow]$ (MGAB).

\section{Distribution}

Palaearctic.

Certain records: Romania (Ionescu 1969: 276). 
Allotria (Auloxysta) nigripes Thomson, 1877: 813. Type: deposited in MZLU (examined).

Allotria nigripes Thomson: Dalla Torre 1893: 34.

Allotria (Bothrioxysta) nigripes - Dalla Torre \& Kieffer 1902: 40.

Auloxysta nigripes - Ashmead 1903: 142.

Charips (Bothrioxysta) nigripes - Dalla Torre \& Kieffer 1910: 268.

Bothrioxysta nigripes - Rohwer \& Fagan 1917: 362.

Phaenoglyphis nigripes - Rohwer \& Fagan 1919: 337.

Charips nigripes - Muesebeck \& Krombein 1951: 607.

Phaenoglyphis (Auloxysta) nigripes - Hellén 1963: 7.

Phaenoglyphis nigripes - Andrews 1978: 95.

\section{Diagnosis}

Phaenoglyphis nigripes is mainly characterized by the absence of notauli and scutellar foveae (Fig. 7.9), and antenna with rhinaria and club shape beginning on F3, F1 subequal to pedicel, F1 longer than F2 and shorter than F3, and F3 shorter than F4 (Fig. 6.15). It is similar to P. laevis Andrews, 1978, but they can be differentiated by the length of the antenna (shorter than the body in P. nigripes but longer in P. laevis) and where the rhinaria begin (on F3 in P. nigripes (Fig. 6.15) but on F5 in P. laevis).

\section{Material examined}

\section{Holotype}

NORWAY: + [TROMS, 20 Km S of Setermoen, along E6 Road, sweeping, 15 Jul. 1999, leg. G. Melika; 4198] (MZLU).

\section{Distribution}

Palaearctic.

Certain records: Norway (Thomson 1877: 813).

Uncertain records: England (Cameron 1889: 57), Finland (Hellén 1963: 8), Ireland (O’Connor \& Nash 1997), Romania (Ionescu 1969: 248; Trandafirescu et al. 2004: 260), Scotland (Cameron 1889: 57), Spain (Torras-Casals 1996: 196).

Phaenoglyphis proximus Belizin, 1966

Figs 6.16, 7.10

Phaenoglyphis proximus Belizin, 1966: 8. Type: deposited in ZIN (examined).

\section{Diagnosis}

Phaenoglyphis proximus is mainly characterized by the presence of notauli; the presence of oval scutellar foveae separated by a thin carina and completely defined (Fig. 7.10); antenna with rhinaria and club shape beginning on F2, F1 subequal to pedicel, F1 longer than F2, and F2-F4 subequal in length (Fig. 6.16). According to these features, there is no other species of Phaenoglyphis very similar to P. proximus. 


\section{Material examined}

\section{Holotype}

MOLDOVA: + [Kishinev, 5.7.60, zatsch. polosa, 129V. Talitzkiy], [Holotypus Phaenoglyphis proximus V. Belizin d. $\mathrm{m}$ + ] (red label) (ZIN).

\section{Distribution}

Palaearctic.

Certain records: Moldova (Belizin 1966: 8).

Phaenoglyphis pubicollis (Thomson, 1877)

Figs 6.17, 7.11

Allotria (Auloxysta) pubicollis Thomson, 1877: 812. Type: deposited in MZLU (examined).

Allotria pubicollis - Dalla Torre 1893: 35.

Hemicrisis pubicollis - Kieffer 1900: 113.

Phaenoglyphis pubicollis - Dalla Torre \& Kieffer 1902: 42.

\section{Diagnosis}

Phaenoglyphis pubicollis is mainly characterized by the presence of notauli that are more evident at the anterior margin; the presence of oval scutellar foveae separated by a carina and open at the bottom (Fig. 7.11); female antenna with rhinaria and club shape beginning on F1, F1 longer than pedicel and F2, and F2-F4 subequal in length (Fig. 6.17). It is similar to P. evenhuisi, but they can be differentiated by where rhinaria begin (F1 in P. pubicollis (Fig. 6.17), but F4 in P. evenhuisi (Fig. 6.8)) and the shape of the notauli (only indicated anteriorly and well-marked posteriorly in P. pubicollis (Fig. 7.11) while only indicated in P. evenhuisi (Fig. 7.2)).

\section{Material examined}

\section{Lectotype}

SWEDEN: $q$ [Lund Åroskut 31/7 1840; pubicollis (handwritten)], [1972, 48], [holotype], [Allotria pubicollis Thomson det. H.H. Evenhuis 1973], [1979, 306], [1983, 754 (green label)], [ZML. 2004, 344 (green label)] (MZLU).

\section{Distribution}

Palaearctic.

Certain records: Sweden (Thomson 1877: 812).

Phaenoglyphis ruficornis (Förster, 1869)

Figs 6.18, 7.12

Hemicrisis ruficornis Förster, 1869: 339. Type: deposited in NMW (examined).

Phaenoglyphis ruficornis - Evenhuis 1973: 219. — Pujade-Villar \& Paretas-Martínez 2006: 479.

Hemicrisis ruficornis - Ronquist 1999: 163. 


\section{Diagnosis}

Phaenoglyphis ruficornis is mainly characterized by the presence of notauli, with a few wrinkles on their distal side; the presence of scutellar foveae that are horizontally elongated, separated by a carina, and completely open at the bottom (Fig. 7.12); antenna with rhinaria and club shape beginning on F1 in both males and females, F1 longer than pedicel and F2, and F2-F4 subequal in length in both males and females (Fig. 6.18). It is similar to P. pubicollis and P. evenhuisi because of their sculpturing. However, they can be differentiated because P. ruficornis has a mostly smooth mesoscutum, with a few wrinkles on the distal side of the notauli (Fig. 7.12), while P. pubicollis and P. evenhuisi have distinctive imbricate sculpturing on all surfaces (Fig. 7.8, 7.11).

\section{Distribution}

Holarctic.

Certain records: Canada (Menke \& Evenhuis 1991: 150), Germany (Förster 1869: 339), USA (Menke \& Evenhuis 1991: 150).

Uncertain records: Israel (Argaman 1988: 115), Japan (Watanabe 1950: 87).

Phaenoglyphis salicis (Cameron, 1883)

Figs 6.19, 7.13

Allotria salicis Cameron, 1883: 367. Type: deposited in BMNH (examined).

Hemicrisis salicis - Cameron 1886: 89.

Phaenoglyphis salicis - Cameron 1890: 237.

\section{Diagnosis}

Phaenoglyphis salicis is mainly characterized by a closed radial cell that is 2.5 times as long as wide; the presence of pronotal and propodeal carinae; the weak presence of notauli; the presence of oval scutellar foveae that are completely defined and with two lines on top (Fig. 7.13); female antenna with rhinaria beginning on F3, F1 longer than pedicel and F2, F2 shorter than F3, and F3 subequal to F4 (Fig. 6.19). It is similar to P. gutierrrezi Andrews, 1978, but they can be differentiated by flagellomere proportions (F2 is shorter than F3 and F3-F4 are subequal in length in P. salicis (Fig. 6.19), while F2-F4 are subequal in length in P. gutierrezi) and the shape of the scutellar foveae (completely defined and with two lines at the top in P. salicis (Fig. 7.13), but slightly open at the bottom in P. gutierrezi).

\section{Material examined}

\section{Holotype}

UNITED KINGDOM: England: $q$ [Holotype (round label with red in the margin)], [Cameron 96-76/ salicis], [217-11-129; Allotria salicis Cameron 1883 \& (orange label)], [BM (red label)], [B.M. TYPE HYM 7178], [HOLOTYPE $q$ of Allotria salicis Cam. det. J. Quinlan 1997], [Phaenoglyphis salicis (Cameron), J. P-V det. 2004/ nr abbreviate (Thomson)] (BMNH B.M. Type Hym 7178).

Additional specimens $(1 \hat{\partial}, 2$ 우)

AUSTRIA: 1 ठ [Leibnitz, R. Mur, 3 Jun. 1969, B.M. 1970-152, B.H. and M.C. Cogan, R.I. and R. VaneWright] (BMNH).

GERMANY: 1 [Rhine Valley near Hügelheim, 4750'10" N, 7³7'9" E, 15 Jun. 1999, H. Goulet, sweeping old alfalfa] (CNCI C-230). 
ITALY: 1 ㅇ [Abruzzo (AQ), L'Aquila, Aterno River, sweeping riparian veg. and Salix, 20 Jun. 1992, J.D. Pinto] (CNCI C-253).

\section{Distribution}

Palaearctic.

Certain records: England (Cameron 1888: 210), Scotland (Cameron 1883: 367).

Uncertain records: Ireland (O’Connor \& Nash 1997), Romania (Ionescu 1969: 274), Spain (TorrasCasals 1996: 196, 197).

New records: Austria, Germany and Italy.

\section{Phaenoglyphis stricta (Thomson, 1877)}

Figs 6.20, 7.14

Allotria (Auloxysta) stricta Thomson, 1877: 812. Type: deposited in MZLU (examined).

Allotria stricta - Dalla Torre 1893: 35.

Phaenoglyphis stricta - Kieffer 1900: 113. — Andrews 1978: 96.

Phaenoglyphis striata - Dalla Torre \& Kieffer 1902: 42 (lapsus).

Phaenoglyphis (Phaenoglyphis) stricta - Hellén 1963: 6.

\section{Diagnosis}

Phaenoglyphis stricta is mainly characterized by scutellar fovea with straight sides and open on the top and on the bottom (Fig. 7.14); female antenna with rhinaria and club shape beginning on the last two thirds of F1, F1 longer than pedicel and F2, and F2-F4 subequal in length (Fig. 6.20). It is similar to $P$. insperatus, but they can be differentiated by the shape of the scutellar foveae (straight sides and open both on the top and on the bottom in P. stricta (Fig. 7.14), but rounded and slightly open at the bottom in P. insperatus (Fig. 7.5)) and the size of the radial cell (2.4 times as long as wide in P. stricta but 2.9 times in P. insperatus).

\section{Material examined}

\section{Lectotype}

SWEDEN: 9 [Skalstugan, Ing. Alp. Jemtl., 16-21 40], [1969, 88 (green label)], [1972, 45], [1979, 307], [Allotria (Auloxysta) stricta Thomson, 1877, redet. E. Kierych 1979], [ZML.2004, 339 (green label)], [Lectotype Allotria (Auloxysta) stricta Thomson, 1877 ㅇ desig. M. Ferrer-Suay 2011 (red label)], [Phaenoglyphis stricta (Thomson, 1877) ㅇ M. Ferrer-Suay det. 2011] (MZLU).

\section{Distribution}

Palaearctic.

Certain records: Andorra (Ferrer-Suay et al. 2011: 360), Mexico (Ferrer-Suay et al. 2013m: 39), Japan (Ferrer-Suay et al. 2013i: 22), Sweden (Thomson 1877: 812).

Uncertain records: Denmark (Hellén 1963: 6), England (Andrews 1978: 96), Finland (Hellén 1963: 6), France (Andrews 1978: 96), Germany (Andrews 1978: 96; Hübner et al. 2002: 507), Russia (Hellén 1963: 6). 
Xystus villosus Hartig, 1841: 353. Type: deposited in ZSM (examined).

Allotria villosus - Taschenberg 1866: 130.

Allotria villosa - Dalla Torre 1893: 36.

Dilyta (Alloxysta) villosa - Kieffer 1900: 114.

Alloxysta (Alloxysta) villosa - Dalla Torre \& Kieffer 1902: 39; 1910: 260.

Phaenoglyphis villosa - Evenhuis \& Barbotin 1977: 184.

Alloxysta villosa - Andrews 1978: 92.

\section{Diagnosis}

Phaenoglyphis villosa is characterized by a partially open radial cell that is $2.1-2.7$ times as long as wide; the presence of pronotal and propodeal carina; the absence of notauli; scutellum with two deep oval foveae more or less separated by a carina or completely fused (Fig. 7.15); female antenna with rhinaria beginning on F3, F1 as long as pedicel or slightly longer, F1 subequal to F2, F2 shorter than F3, and F3 shorter than F4 (Fig. 6.21); male antenna with rhinaria beginning on F3, F1 subequal to $\mathrm{F} 2$, and F2 shorter than F3. At the moment, $P$. villosa is easily differentiated from the other species of Phaenoglyphis, because it is the only one with a partially open radial cell.

\section{Material examined}

\section{Holotype}

GERMANY: $q$ [o; Holotype (orange label); Xystus villosus Hartig, det. H.H. Evenhuis 1977; Phaenoglyphis villosa (Hartig, 1841) 9 M. Ferrer-Suay det. 2011] (ZSM).

Additional specimens $(28 \hat{\partial} \widehat{\partial}, 64$ 우 $)$

CYPRUS: 1 §ึ, 2 우 [Limassol, 25 May 1934, G.A. Mavromoustakis] (BMNH B.M. 1935-55).

CZECH REPUBLIC: 2 우 [central Bohemia, 30 Jul. 1991, J. Macek] (CNCI C-278); 1 임oravia, Lanzhot-Ranspurk, 7-9 Aug. 1991, L. Masner, sweep, climax flood forest] (CNCI C-268); 1 ㅇ [Moravia, 16 km N Blansko, 8 Aug. 1991, L. Masner, sweep. Tilia-Acer forest] (CNCI C-271); 1 q [Moravia, Javorina, 800-900 m, 10 Aug. 1991, L. Masner, sweep, climax deciduous forest] (CNCI C-266); 1 ๙ [Moravia, Zidlochovice, 20 Nov. 1984, N.D. Springate] (BMNH).

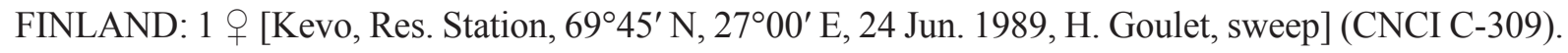

FRANCE: 1 [ [Hérault, Baillarguet CSIRO lab., 4341'12" N, 352'24" E, 15-22 May 1993, P.G. Mason, champ sauvage, MT] (CNCI C-302).

GERMANY: 1 q [Mainz, 18 Sep.-1 Oct. 1965, A.W. Steffan] (CNCI C-236); 1 q [Ingelheim am Rhein, ??.??.1968, MT in orchard, A.W. Steffan] (CNCI C-238); 2 우 [Ingelheim am Rhein, 1-30 Sep. 1968, MT, A.W. Stephan, orchard, cynipoidea] (CNCI C-237); 3 $\widehat{\jmath}, 3$ 우 [Schwarzwald, Todtmoos environs, 6 Aug. 1984, L. Masner, screen sweeping] (CNCI C-235); 1 ऽ [Anmuhle, near Hamburg Sachsenwald, 22 Aug. 1984, L. Masner, screen sweeping] (CNCI C-296).

IRAN: 3 q $q$ [Karaj, 40 km W Tehran, 25-28 Jun. 1978, J.T. Huber, YPT] (CNCI C-316); 8 q $q$ [Karaj, 40 km W Tehran, 3 Jul. 1978, J.T. Huber sweep] (CNCI C-315).

JAPAN: 1 \& [Ibaraki, Tsukuba, NIAES, 14-21 Jul. 1989, M.J. Sharkey, PT] (CNCI C-191); 1 q [Ibaraki, Tsukuba, NIAES, 31 May-8 Jun. 1989, M.J. Sharkey, PT] (CNCI C-195); 1 ð [Hokkaido, 20 km N. 
Akkeshi, marsh, 100 m, 15 Jul. 1996, L. Masner, sweep] (CNCI C-160); 3 ふ઼ [Fukuoka, Mt. Hiko, 700 m, 9-10 May 1989, M.J. Sharkey, sweep] (CNCI C-183); 1 ㅇ [Ibaraki, Tsukuba, NIAES, 8-15 Jun. 1989, M. Sharkey, FIT and MT] (CNCI C-150); 1 \& [Ibaraki, Tsukuba, NIAES, 27 Oct.-13 Nov.

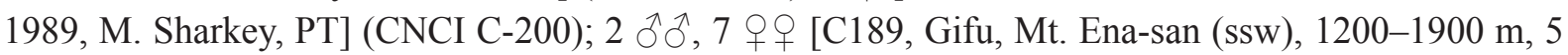
Jun. 1995, K. Yamagishi]; 1 \& [Ibaraki, Tsukuba, Expo site, 7-16 May 1989, M.J. Sharkey, PT] (CNCI C-135); 1 $\widehat{~[G i f u, ~ K a s a g a w a, ~ I k e d a-c h o, ~} 24$ May 1984, R. Baczyuski] (CNCI C-181).

MALTA: 1 q [Gozo, Ghasri Valley, 28 Dec. 1970, R.I. Vane-Wright] (BMNH B.M. 1971-38).

MOROCCO: 3 우우 [Marrakech, Ouirgane, $1000 \mathrm{~m}, 31^{\circ} 08^{\prime} \mathrm{N}, 8^{\circ} 05^{\prime} \mathrm{W}, 8-13$ May 1996, C. Kassebeer, MT] (CNCI C-295); 1 q [Marrakech, Ouirgane, 1000 m, 31 $08^{\prime}$ N, $8^{\circ} 05^{\prime}$ W, 4-11 Oct. 1996, C. Kassebeer, MT] (CNCI C-279).

PALESTINE: 1 [Rfar Ano, 25 Jul. 1950, ex Dacus olea, leg. Bylinski-Solz, 3167, Com. Inst. Ent. Coll. No. 2247] (BMNH).

RUSSIA: 1 ð, 2 우 [Primoskiy Kray Ussyriysk District, Gomotayozhnoye, 11-15 Aug. 2003, Malaise trap, M.V. Michailovskaya] (USNM).

SLOVAKIA: 1 q [Moravia, Lanzhot-Ranspurk, 9-12 Aug. 1991, L. Masner, climax hardwood forest, PT] (CNCI C-270); 1 [Košice, 14-17 Sep. 1984, N.D. Springate] (BMNH).

SLOVENIA: 1 q [Radovljica, 3 Aug. 1978, L. Huggert, swept on dry meadow] (CNCI C-276).

SWEDEN: 2 q [Sk., Lund Zoological Mus. grounds, Aug. 1976] (BMNH); 1 [ [Kalmar, Överum, 19 May-29 Jun. 1978, L. Huggert, MT at swampy lake shore] (CNCI C-312).

SWITZERLAND: 13 ふึ̊ึ, 10 qq [Dielsdorf, 650 m, 17 Aug. 1984, L. Masner, sweep] (CNCI C-290).

TURKEY: 1 đ̊ [Bornova-Izmir, 4 May 1976, E. Fakültesi (handwritten), Ex. Myzus persicae] (BMNH).

UNITED KINGDOM: England: 1 \& [Lincolnshire, Moorside near Fulsby Wood, 22-25 Aug. 1986, $53^{\circ} 7^{\prime} 6^{\prime \prime} \mathrm{N}, 0^{\circ} 7^{\prime} 11^{\prime \prime} \mathrm{W}, \mathrm{J} . \mathrm{S}$. Noyes] (CNCI C-277).

\section{Distribution}

Cosmopolitan.

Certain records: Andorra (Pujade-Villar et al. 2007: 171), Argentina (Pujade-Villar et al. 2002: 543), Balearic Islands (Pujade-Villar et al. 2001: 85), Belgium (Pujade-Villar et al. 2007: 171), Canada (Menke \& Evenhuis 1991: 150; Pujade-Villar et al. 2007: 171), Chile (Pujade-Villar et al. 2002: 543), China (Pujade-Villar et al. 2007: 171), Colombia (Ferrer-Suay et al. 2012f: 327), England (Müller et al. 1999: 346), Germany (Pujade-Villar et al. 2007: 171), Greece (Pujade-Villar et al. 2007: 171), Hungary (Pujade-Villar et al. 2007: 171), India (Ferrer-Suay et al. 2013i), Iran (Pujade-Villar et al. 2007: 171), Japan (Pujade-Villar et al. 2007: 171; Takada \& Nakamura 2010: 270), Madeira (Borges et al. 2008; Ferrer-Suay et al. 2012g: 14), Mexico (Ferrer-Suay et al. 2013m: 40), Morocco (Pujade-Villar et al. 2007: 171), New Zealand (Valentine 1975: 60; Evenhuis \& Barbotin 1977: 185; Ferrer-Suay et al. 2012h: 238), Norway (Pujade-Villar et al. 2007: 171), Taiwan (Pujade-Villar et al. 2007: 171), The Netherlands (Evenhuis \& Barbotin 1977: 185), Ukraine (Pujade-Villar et al. 2007: 171), USA (PujadeVillar et al. 2007: 171). 
Uncertain records: Algeria (Kieffer 1909: 482), Australia (Girault 1931: 2; Carver 1992: 783; Wilson \& Swincer 1984: 47), Belgium (Crèvecoeur \& Maréchal 1933: 269), Bulgaria and Balkan Peninsula (Vasileva-Sumnalieva 1976: 24), Canada (Andrews 1978: 34), England (Cameron 1889: 58; Dalla Torre \& Kieffer 1910: 280), Finland (Hellén 1931: 5; 1958: 67; 1963: 7), France (Kieffer 1902a: 11-13; 1902b: 595, 597; De Gaulle 1908: 26; Andrews 1978: 84), Germany (Hartig 1841: 353; Hübner et al. 2002: 509), South Africa (Gaston et al. 2003: 1096), Hawaii (Beardsley 1985: 50), Iceland (Weld 1952: 253), Iraq (Al-Jassani \& Al-Adil 1986: 59), Ireland (O’Connor \& Nash 1997), USA (Ashmead 1898: 156), Moldova (Belizin 1966: 7), Poland (Kierych 1979b: 14), Romania (Ionescu 1963: 174; 1969: 247; Prelipcean et al. 2004: 60), Russia (Belizin 1962: 126; 1973: 36; Hellén 1963: 7), Peninsular Spain (Torras-Casals 1996: 196-197; Suay et al. 1998: 106), Sweden (Thomson 1862: 409), USA (Andrews 1978: 34; Menke \& Evenhuis 1991: 150), USA (Kieffer 1909: 481), USA (Andrews 1978: 34).

New records: Cyprus, Czech Republic, Morocco, Palestine, Turkey and Switzerland.

\section{Phaenoglyphis xanthochroa Förster, 1869}

Figs 6.22, 7.16

Phaenoglyphis xanthochroa Förster, 1869: 339. Type: deposited in ZMHB (examined).

Phaenoglyphis (Phaenoglyphis) xanthochroa - Hellén 1963: 6.

Phaenoglyphis xanthochroa - Andrews 1978: 96.

\section{Diagnosis}

Phaenoglyphis xanthochroa is easily differentiated from the other species of Phaenoglyphis by its dark yellow body and its deeply excavated notauli.

\section{Material examined}

\section{Lectotype}

GERMANY: $q$ [Phaenoglyphis xanthochroa $q$ III (handwritten)], [Zool. Mus. Berlin], [Weld 1931 (rounded red label)], [Lüttich (handwritten)], [Lectotype Phaenoglyphis xanthochroa Förster, 1869 q desig. M. Ferrer-Suay 2011 (red label)], [Phaenoglyphis xanthochroa Förster, 1869 M. Ferrer-Suay det. 2011] (ZMHB).

Additional specimens ( $4 \hat{\partial} \widehat{\partial}, 10$ 우)

CZECH REPUBLIC: 2 $\widehat{\jmath}, 3$ 우 [Moravia, 16 km N Blansko, 8 Aug. 1991, L. Masner, sweep, TiliaAcer forest] (CNCI C-271).

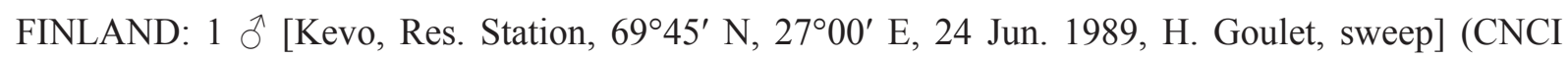
C-309).

GERMANY: 1 §ึ, 2 우우 [Schwarzwald, Todtmoos environs, 6 Aug. 1984, L. Masner, screen sweeping] (CNCI C-235).

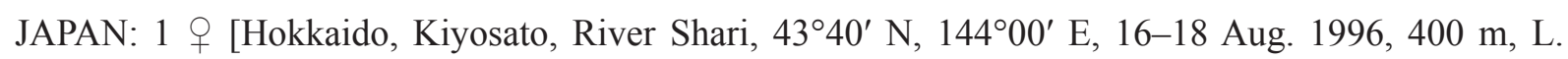
Masner, YPT, forest] (CNCI C-165); 1 \& [Hokkaido, Sapporo, Jozankei, 350 m, 20-31 Jul. 1989, K. Maeto and M. Sharkey] (CNCI C-151).

SWITZERLAND: 2 우 [Jura, Delémont, CABI Lab. 47²2'42" N 7²0'30" E, 7 Jun. 1999, H. Goulet, spruce/beech] (CNCI C-287); 1 q [Dielsdorf, 650 m, 17 Aug. 1984, L. Masner, sweep] (CNCI C-290). 


\section{Distribution}

Palaearctic.

Certain records: England (Müller et al. 1999: 346), Germany (Förster 1869: 339), The Netherlands (Evenhuis 1978: 171), Japan (Ferrer-Suay et al. 2013i).

Uncertain records: England (Marshall 1870; Kieffer 1902b: 14), Finland (Hellén 1963: 6), France (De Gaulle 1908: 26), Ireland (O’Connor \& Nash 1997), Poland (Krawczyk et al. 2009: 163), Sweden (Thomson 1877: 812).

New records: Czech Republic, Japan, and Switzerland.

\section{Discussion}

The importance of revising old entomological collections is demonstrated here. Thanks to this work, new information has appeared and helped us to improve our knowledge about an important group of parasitoid wasps. This is the last revision of the subfamily Charipinae as a part of a series of papers compiling revisions at a worldwide level. Papers focusing on each different biogeographic region have already been published: Oriental (Ferrer-Suay et al. 2013i), Australia (Ferrer-Suay et al. 2014d), Africa (Ferrer-Suay et al. 2013j), Neotropical (Ferrer-Suay et al. 2013k) and Nearctic (Ferrer-Suay et al. 2014a).

This is the first time that a revision of Charipinae from the Palaearctic region has been done, concerning the entire Palaearctic region and with updated data. This region is relatively well-known for Charipinae compared to other regions. Most of the species described came from the Palaearctic. However, even so, in this work 33 species have been cited for the first time from different countries, and two new species have been found.

Up to now, the species boundaries have been based on morphological features. However, sometimes these features are not sufficiently reliable to delimit species, and we need to test their reliability. For this reason, it is neccesary to complete these faunistic studies with molecular markers. This way, with a combination of morphological and molecular markers, we can be almost completely sure of the species boundaries. This line of research is currently underway.

Charipinae are known to be harmful for aphid biological control programs. They have an adverse effect on the performance of the primary parasitoids by decreasing their abundance and modifying their behaviour (Sampaio et al. 2017). Host specificity of these hyperparasitoids is still under debate, and for many Charipinae species very little is known about their trophic relationships. One view is that polyphagy in the Charipinae is limited to a few species, whereas most species are specialized to a variable extent, with specialization on a certain aphid and primary parasitoid combination at the extreme end (Evenhuis 1976; Sullivan \& Völk1 1999). The opposite view is held by Fergusson (1986), who considered the Charipinae to contain relatively few species that are morphologically variable or fairly polyphagous. DNA analysis of one species group indicated that the latter point of view is incorrect and that Charipinae are more diverse and more specialized (van Veen et al. 2003). Until now, only one study (Ferrer-Suay et al. 2014f) has investigated the specificity of species of Charipinae, and that study represents the first qualitative analysis of host specificity and host overlap in the subfamily Charipinae. It focused on the cosmopolitan genus Alloxysta, for which many of the hosts are known (Ferrer-Suay et al. 2014f). According to this study, within this subfamily, A. arcuata, A. brevis, A. fuscicornis and A. victrix are the most generalist species that share many aphid hosts, while for primary parasitoid hosts the most generalist species are: A. arcuata, A. brevis, A. pleuralis and A. victrix. Alloxysta citripes, A. halterata, A. leunisii and A. ramulifera appear, up to now, as the most specialized in relation to primary parasitoid hosts. 
The Charipinae is a cosmopolitan subfamily and well distributed around the six biogeographical regions, according to our records. They are of course limited in their distribution to those places where aphids and their primary parasitoids are found. We could infer that the Charipinae has a continuous distribution, and the gaps found between the regions are possibly due to the fact that it has not been studied or collected in those places.

It will be necessary to continue with studies on Charipinae, collecting new material as well as revising old collections or material collected by different research groups. This way, the original distribution patterns of species can be elucidated and improved. This work is presented as a useful tool for those who have to identify Charipinae in the Palaearctic region.

Although knowledge of taxonomy on Charipinae has been greatly improved, some points are still under debate and need special attention in the future. The molecular knowledge on this subfamily is still very scarce, with sequences available only for some of the common species. Future research should concentrate on expanding molecular analysis in order to improve our knowledge of the group's diversity, species limits, evolution, biogeography, etc. Another important point under debate within this subfamily is the paraphyly of the genus Phaenoglyphis, the basal genus of the subfamily Charipinae. Once the morphological and molecular knowledge of this subfamily is complete, the next step will be to solve its internal phylogeny. This important step will help us to determine the limits between species and to see if the morphological limits established up to now correspond to the molecular limits. Additionally, it will be necessary to improve knowledge about the trophic associations of Charipinae, to collect material in the field, and to establish the relationships between hosts and parasitoids. This way, we would have useful information for aphid management programs.

\section{Acknowledgements}

We are very grateful to John Huber for allowing us to study all the Malaise trap material in alcohol deposited at the Canadian National Collection of Insects (CNCI), where we found the specimens used for this study. We also want to thank David Notton and Matthew Buffington for their hospitality during our stays at the Natural History Museum (London, United Kingdom) and the United States National Museum of Natural History (Smithsonian Institution) (Washington, United States), respectively. The following people are acknowledged for providing information about the current location of some type specimens: David Notton (Natural History Museum, UK); Jennifer Read and John Huber (CNCI, Canada), Juho Paukkunen, Pekka Malinen, Olof Biström and Laura Hiisivuori (Zoological Museum at the Finnish Museum of Natural History); Matthew Buffington (National Museum of Natural History, Washington); Roy Danielsson (Lund University Museum, Sweden); Melania Stan (Muzeul de Istoria Naturala, "Grigore Antipa", Bucharest, Romania); Catriona McPhee (National Museum of Victoria, Australia); Sergey Belokobylskij (Zoological Institute of the Russian Academy of Sciences, Russia) and Frank Koch (Zoologisches Museum Humboldt-Universität, Berlin, Germany). This research was supported by the projects CGL2008-00180 and CGL2011-22889 of the Ministerio de Ciencia e Innovación (Spain) and the grant AP2009-4833 of the Ministerio de Educación (Spain).

\section{References}

Ahmad Md.E. \& Singh R. 1996. Records of aphid parasitoids from the Bihar and associations with their hosts and food plants. Journal of Advanced Zoology 17 (1): 26-33.

Al-Jassani R.F. \& Al-Adil K.M. 1986. Insect enemies of the black bean aphid Aphis fabae Scop. in AbuGraib. Journal of Biological Sciences Research 48: 812-813. 
Andrews F.G. 1978. Taxonomy and host specificity of Nearctic Alloxystinae with a catalogue of the World species (Hymenoptera: Cynipidae). Ocassional Papers in Entomology 25: 1-128.

Archimowitsch A. 1952. Fauna de insectos en España que acuden a los "bytagranos" de la remolacha. Boletín de la Sociedad Española de Historia Natural, Serie biológica L: 91.

Argaman Q. 1988. Additions to the Cynipoid fauna of Israel (Hymenoptera, Cynipoidea). Israel Journal of Entomology 22: 109-117.

Ashmead W.H. 1887. Report on insects injurious to garden crops in Florida. U.S. Department of Agriculture, Division of Entomology, Bulletin 14: 9-29.

Ashmead W.H. 1898. Descriptions of new parasitic Hymenoptera. Proceedings of the U.S. Department of Agriculture, Entomology 4: 155-171.

Ashmead W.H. 1903. Classification of the gall-wasps and the parasitic Cynipoids, or the superfamily Cynipoidea III. Psyche 10: 140-155.

Baker C.F. 1896. New American parasitic Cynipidae (Allotriinae). Canadian Entomologist 28: 131-135.

Barczak T. 1991. The alloxystids as hyperparasitoids of the Aphis fabae group in Poland (Hym., Cynipoidea: Alloxystidae; Hom.: Aphididae). Poliskie Pismo Entomologiczne 61: 85-95.

Barnea O., Mustata M., Mustata G. \& Feraru E. 2005. The complex of parasitoids controling some colonies of aphids. Lucrările simpozionului Entomofagii şi rolul lor în păstrarea echilibrului natural. Universitatea "Al I. Cuza”, Iaşi.

Beardsley J.W. 1985. Notes on Hawaiian Alloxystidae and Cynipidae (Hymenoptera: Cynipoidea). Proceedings of the Hawaiian Entomological Society 25: 49-52.

Belizin V.I. 1962. New Parasitoid Cynipoidea species (Hymenoptera) from the Far East. Communications of the Far East Branch of the Russian Academy of Sciences (Siberian Section) 16: 125-129.

Belizin V.I. 1966. Paraziticheskie tsinipidy (Hymenoptera, Cynipoidea) moldavskoj SSR [Parasitic Cynipids (Hymenoptera, Cynipoidea) in the Moldavian SSR]. Trudy Moldavskoho nauchono-issled. Instituta Sadovodstva, Vinogradarstva i Vinodelija (Entomologia) 13: 1-14.

Belizin V.I. 1968. New genera and species of gall wasps (Hymenoptera, Cynipoidea) of the Soviet Far East and adjacent territories. District Station of Plant Protection (Kursk) 5: 701-719.

Belizin V.I. 1973. New cynipids (Hymenoptera, Cynipoidea) from the USSR and neighbouring countries. Revue d'Entomologie de l'URSS 52 (1): 29-38.

Berta D.C., Colomo M.V. \& Ovruski N.E. 2002. Interrelaciones entre los áfidos colonizadores del tomate y sus himenópteros parasitoides en Tucamán (Argentina). Boletin de Sanidad Vegetal Plagas 28: 67-77.

Bertolaccini I., Núñez-Pérez E. \& Tizado E.J. 2004. Plantas hospedadoras alternativas de áfidos plaga de cultivos de leguminosas, sus parasitoides e hiperparasitoides en la provincia de León (Spain). Boletin de la Asociación española de Entomologia 28 (3-4): 33-47.

Betini A. 1975. Afideos da macieire (Pyrus malus L.), seus predadores e parasitas. Acta Biologica Paranaense, Curitiba 4 (3-4): 33-74.

Betini A. 1976. Afideos da ameixeira (Prunus domestica L.) e pessegueiro (Prunus persica sto.), seus predadores e parasitas. Acta Biologica Paranaense, Curitiba 5 (1-2): 69-90.

Bokina I.G. 1997. Hyperparasites of grain aphids in forest steppe of the northern Ob river basin in West Siberia. Zoologicheskii Zhurnal 76 (4): 432-437.

Borges P.A.V., Abreu C., Aguiar A.M.F., Carvalho P., Jardim R., Melo I., Oliveira P., Sérgio C., Serrano A.R.M. \& Vieira P. 2008. A List of the Terrestrial Fungi, Flora and Fauna of Madeira and Selvagens 
Archipelagos. Direcção Regional do Ambiente de Madeira and Universidade dos Açores, Funchal and Angra do Heroísmo.

Bouchard D., Tourneur J.C. \& Paradis R.O. 1982. Les parasitoides du puceron vert du pommier Aphis pomi de Geer, dans le sud-ouest du Quebec. Annales de la Société entomologique du Québec 27 (3): 184-190.

Buhl P.N. 1997. Microhymenoptera from Zackenberg, North East Greenland (Hymenoptera: Chalcidoidea, Cynipoidea et Ceraphronoidea). Entomologiske Meddelelser 65: 161-164.

Cameron P. 1879. On some new or little known British Hymenoptera. Transactions of the Entomological Society of London 1879: 107-119. https://doi.org/10.1111/j.1365-2311.1879.tb01980.x

Cameron P. 1883. Descriptions of sixteen new species of parasitic Cynipidae, chiefly from Scotland. Transactions of the Entomological Society of London 16 (4): 365-374.

https://doi.org/10.1111/j.1365-2311.1883.tb02952.x

Cameron P. 1886. The fauna of Scotland, with special reference to Clydesdale and the western district. Proceedings of the Natural History Society of Glasgow 3: 53-95.

Cameron P. 1888. On some new or little known British parasitic Cynipidae. Entomologist's Monthly Magazine 24: 209-211.

Cameron P. 1889. On the British species of Allotrinae, with descriptions of other new species of parasitic Cynipidae. Memoirs of Manchester Literary and Philosophical Society 2: 53-69.

Cameron P. 1890. A Monograph of the British Phytophagous Hymenoptera. Volume III. Ray Society, London.

Carver M. 1992. Alloxystinae (Hymenoptera, Cynipoidea, Charipidae) in Australia. Invertebrate Taxonomy 6 (3): 769-785.

Cavro E. 1954. Catalogue des Hyménoptères du département du Nord et des régions limithrophes. III. Térébrants (Parasites byte tarières). Bulletin de la Société entomologique du Nord de la France 75 (suppl.): 1-134.

Ceballos G. 1941. Las tribus de los Himenópteros de España. Consejo Superior de Investigaciones Científicas, Instituto español de Entomologia, Madrid.

Chua T.H. 1978. Pattern and influencing factors or emergence in Diaretiella rapae and its parasites. Zeitschrift für angewandte Entomologie 85 (4): 436-442.

Cividanes F.J. 2002. Impacto de inimigos naturais e de fatores meteorológicos sobre uma populaçao de Brevicoryne brassicae (L.) (Hemiptera: Aphididae) em couve. Neotropical Entomology 31 (2): 249-255.

Crèvecoeur A. \& Maréchal P. 1933. Matériaux pour servir à l'établissement d'un nouveau catalogue des Hyménoptères de Belgique IV. Bulletin et Annales de la Société royale entomologique de Belgique 73 (11): 373-382.

Curtis J. 1838. British Entomology; being Illustrations and Descriptions of the Genera of Insects found in Great Britain and Ireland: Containing Coloured Figures of Nature of the Most Rare and Beautiful Species and in Many Instances of the Plants upon which they are found 15: 674-721. Privately published, London.

Dahlbom G. 1842. Onychia och Callaspodia. Berling, Lund.

Dalla Torre C.G. 1893. Catalogus Hymenopterorum, Vol. II: Cynipiden. Englemann, Lipsiae.

Dalla Torre K.W. \& Kieffer J.J. 1902. Cynipidae. In: Wytsman P. (ed.) Genera Insectorum, Vol. I. Desemt-Verteneuil, Brussels. 
Dalla Torre K.W. \& Kieffer J.J. 1910. Das Tierreich XXIV: Cynipidae. R. Friedlander \& Sons, Berlin.

De Gaulle J. 1908. Catalogue Systématique \& Biologique des Hyménoptères de France. Librairie Paul Klincksieck, Paris.

De Santis L. 1937. El hiperparásito del pulgón verde de los cereales. In: Lopez Cristobal (ed.) Los "Pulgones verdes" de los cereales y sus parásits 3: 14-16. La Plata Univ.

Dessart P. 1969. Les types de Cynipidae décrits par l'abbé Jean-Jaques KIEFFER, conservés dans la collection Léon CARPENTIER au Musée d'Histoire naturelle d'Amiens (France). Bulletin et Annales de la Société royale entomologique de Belgique 105: 180-201.

Dettmer H. 1925. Neue Cynipiden aus den Niederlanden. Natuurhistorisch Maandblad Maastricht 14: $122-125$.

Dunn J.A., 1949. The parasites and predators of potato aphids. Bulletin of Entomological Research 40 (1): 97-122. https://doi.org/10.1017/S0007485300022744

Evans G.A. \& Stange L.A. 1997. Parasitoids associated with the brown citrus aphid, Toxoptera citricida, in Florida (Insecta: Hymenoptera). Entomological Circular 384: 1-5.

Evenhuis H.H. 1973. Studies on Cynipidae Alloxystinae. 3. The identity of Phaenoglyphis ruficornis (Förster, 1869) comb. nov. Entomologische Berichten 33: 218-219.

Evenhuis H.H. 1974. Studies on Cynipidae Alloxystinae 4. Alloxysta macrophadna (Hartig, 1841) and Alloxysta brassicae (Ashmead, 1887). Entomologische Berichten 34: 165-168.

Evenhuis H.H. 1976. Studies on Cynipidae Alloxystinae 5. Alloxysta citripes (Thomson) and Alloxysta ligustri sp. nov., with remarks on host specifity in the subfamily. Entomologische Berichten 36: 140-144.

Evenhuis H.H. 1978. Studies on Cynipidae Alloxystinae 7. Remarks on Cameron's species and a discussion of Phaenoglyphis species with incomplete parapsidal furrows. Entomologische Berichten 38: $169-175$.

Evenhuis H.H. 1982. A study of Hartig's Xystus species with type designations and new synonyms (Hymenoptera: Cynipidae Alloxystinae and Charipinae). Spixiana 5: 19-29.

Evenhuis H.H. \& Barbotin F. 1977. Studies on Cynipidae Alloxystinae. 6. Phaenoglyphis villosa (Hartig) and Alloxysta arcuata (Kieffer). Entomologische Berichten 37: 184-190.

Evenhuis H.H. \& Kiriak I.G. 1985. Studies on Alloxystidae (Hymenoptera, Cynipoidea) 8. Cynips minuta Zetterstedt and Xystus minutus Hartig. Entomologische Berichten 45: 16-20.

Fabianus F. 1900. Liste de quelques Hyménoptères capturés à Malonne. Revue mensuelle de la Société entomologique namuroise 4: 23-27.

Feraru E. \& Mustata G. 2005. Species of parasitoids that control the populations of aphids (Homoptera: Aphididae) from some orchards of Iasi and Vaslui counties. Lucrările simpozionului Entomofagii şi rolul lor în păstrarea echilibrului natural, Universitatea "Al I. Cuza”, Iaşi.

Feraru E., Mustata G. \& Barnea O. 2005. The diversity of the parasitoids in some colonies of Aphids (Homoptera: Aphididae) installed on grassy plants. Lucrările simpozionului Entomofagii şi rolul lor în păstrarea echilibrului natural, Universitatea "Al I. Cuza”, Iaşi.

Fergusson N.D.M. 1986. Charipidae, Ibaliidae and Figitidae (Hymenoptera: Cynipoidea). Handbooks for the Identification of British Insects 8 (1c): 1-55.

Ferrer-Suay M., Selfa J. \& Pujade-Villar J. 2011. Nuevos registros de la subfamilia Charipinae (Hymenoptera, Cynipoidea, Figitidae) para Andorra junto con una clave identificativa. Boletín de la Asociación Española de Entomología 35 (3-4): 345-367.

Available from https://dialnet.unirioja.es/servlet/articulo?codigo=3966356 [accessed 21 Mar. 2018]. 
Ferrer-Suay M., Paretas-Martínez J., Selfa J. \& Pujade-Villar J. 2012a. Taxonomic and synonymic world catalogue of the Charipinae and notes about this subfamily (Hymenoptera: Cynipoidea: Figitidae). Zootaxa 3376: 1-92. Available from http://www.mapress.com/zootaxa/list/2012/3376.html [accessed 21 Mar. 2018].

Ferrer-Suay M., Selfa J., Ribes A. \& Pujade-Villar J. 2012c. Contribucions al coneixement dels Charipins de Catalunya (Insecta, Hymenoptera). Orsis 26: 117-138.

Available from http://www.raco.cat/index.php/Orsis/article/view/254194 [accessed 21 March 2018].

Ferrer-Suay M., Selfa J. \& Pujade-Villar J. 2012d. Revision of V.I. Belizin's type material of Alloxysta (Hymenoptera: Figitidae: Charipinae) deposited in the Zoological Institute of the Russian Academy of Sciences. Zoosystematica Rossica 21 (2): 279-290.

Ferrer-Suay M., Selfa J. \& Pujade-Villar J. 2012e. Revision of the type material of Ionescu collection related to Charipinae subfamily (Hymenoptera: Figitidae) deposited in the "Grigore Antipa" National Museum of Natural History (Bucharest). Travaux du Muséum d'Histoire naturelle "Grigore Antipa" 55 (2): 277-284. https://doi.org/10.2478/v10191-012-0017-7

Ferrer-Suay M., Selfa J. \& Pujade-Villar J. 2012f. Charipinos de Colombia (Hymenoptera: Figitidae), con la descripción de dos nuevas especies. Revista Colombiana de Entomología 38 (2): 320-328.

Ferrer-Suay M., Selfa J. \& Pujade-Villar J. 2012g. Revision of Charipinae (Hymenoptera: Cynipoidea: Figitidae) from Madeira and first record of Alloxysta from Portugal. Boletim do Museu Municipal do Funchal 62 (332): 5-17.

Ferrer-Suay M., Paretas-Martínez J., Selfa J. \& Pujade-Villar J. 2012h. Charipinae fauna from New Zealand with descriptions of two new species of Alloxysta Förster (Hymenoptera: Cynipoidea: Figitidae: Charipinae). Australian Journal of Entomology 51: 229- 238.

https://doi.org/10.1111/j.1440-6055.2012.00859.x

Ferrer-Suay M., Selfa J., Seco-Fernández M.V., Melika G., Alipour A., Rakhshani E., Talebi A.A. \& Pujade-Villar J. 2013a. A contribution to the knowledge of Charipinae (Hymenoptera: Cynipoidea: Figitidae) associated with aphids from Iran, including new records. North-Western Journal of Zoology 9 (1): 30-44.

Ferrer-Suay M., Selfa J., Tomanović Z., Janković M., Kos K., Rakhshani E. \& Pujade-Villar J. 2013b. Revision of Alloxysta from the north-western Balkan Peninsula with description of two new species (Hymenoptera: Figitidae: Charipinae). Acta Entomologica Musei Nationalis Pragae 53 (1): 347-368.

Ferrer-Suay M., Selfa J., Notton D. \& Pujade-Villar J. 2013c. Revision of the types of species of Alloxysta Förster, 1869 described by Cameron and Fergusson (Hymenoptera: Figitidae: Charipinae) deposited in the Natural History Museum (London) including a key to Alloxysta species of the Great Britain fauna. European Jounal of Taxonomy 53: 1-27. https://doi.org/10.5852/ejt.2013.53

Ferrer-Suay M., Selfa J. \& Pujade-Villar J. 2013d. Revision of Thomson and Zetterstedt collections of Alloxysta genus deposited in Lund Museum of Zoology (Sweden). Entomologisk Tidskrift 134: 77-102.

Ferrer-Suay M., Selfa J. \& Pujade-Villar J. 2013e. Revision of Curtis collection of Alloxysta (Hymenoptera: Figitidae: Charipinae) deposited in National Museum of Victoria (Australia). Memoirs of Museum Victoria 70: 11-16.

Ferrer-Suay M., Selfa J., Ribes A. \& Pujade-Villar J. 2013g. A key of the Charipinae (Hymenoptera, Cynipoidea, Figitidae) from Spain, including new records and species. Boletín de la Sociedad Española de Entomología 37 (3-4): 315-341.

Ferrer-Suay M., Selfa J. \& Pujade-Villar J. 2013h. The Alloxysta (Hymenoptera: Figitidae: Charipinae) type material in the National Museum of Natural History and the Canadian National Collection of Insects. The Canadian Entomologist 145 (6): 603-625. https://doi.org/10.4039/tce.2013.52 
Ferrer-Suay M., Selfa J. \& Pujade-Villar J. 2013i. Charipinae fauna (Hymenoptera: Figitidae) from Asia, with description of eleven new species. Zoological Studies 52: 41.

https://doi.org/10.1186/1810-522X-52-41

Ferrer-Suay M., Selfa J. \& Pujade-Villar J. 2013j. A review of Alloxysta species (Hymenoptera: Cynipoidea: Figitidae: Charipinae) from Africa. African Entomology 21 (2): 255-266.

Ferrer-Suay M., Selfa J. \& Pujade-Villar J. 2013k. Review of the Neotropical Charipinae (Hymenoptera, Cynipoidea, Figitidae). Revista Brasileira de Entomologia 57 (3): 279-299. https://doi.org/10.1590/S0085-56262013005000020

Ferrer-Suay M., Selfa J., Villeman C., Andreï-Ruiz M.C. \& Pujade-Villar J. 20131. First record of Charipinae (Hymenoptera: Cynipoidea: Figitidae) from the Corsica Island. Redia XCVI: 3-8.

Ferrer-Suay M., Selfa J., Equihua-Martínez A., Estrada-Venegas E., Lomeli-Flores R., Peña Martínez R. \& Pujade-Villar J. 2013m. Charipinae (Hymenoptera: Cynipoidea: Figitidae) from Mexico with description of three new species. Annals of the Entomological Society of America 106 (1): 26-41. https://doi.org/10.1603/AN12022

Ferrer-Suay M., Selfa J. \& Pujade-Villar J. 2014a. First records, new species and a key of the Charipinae (Hymenoptera: Cynipoidea: Figitidae) from the Nearctic region. Annals of the Entomological Society of America 107 (1): 50-73. https://doi.org/10.1603/AN13077

Ferrer-Suay M., Selfa J. \& Pujade-Villar J. 2014b. New Charipinae (Hymenoptera: Cynipoidea: Figitidae) from Italy. Redia XCVII: 3-13.

Ferrer-Suay M., Selfa J., Seco M.V. \& Pujade-Villar J. 2014c. Revision of Hellén types of Alloxysta Förster (Hymenoptera: Figitidae, Charipinae). Entomologica Fennica 25: 86-101.

Ferrer-Suay M., Selfa J. \& Pujade-Villar J. 2014d. New Australasian records of Alloxysta Förster (Hymenoptera: Cynipoidea: Figitidae: Charipinae) from the Canadian National Collection of Insects, Ottawa. Australian Entomologist 41 (2): 91-106.

Ferrer-Suay M., Selfa J. \& Pujade-Villar J. 2014e. New Charipinae (Hymenoptera: Cynipoidea: Figitidae) from Italy. Redia XCVII: 3-13.

Ferrer-Suay M., Janković M., Selfa J., van Veen F., Tomanović Z., Kos K., Rakhshani E. \& PujadeVillar J. 2014f. Qualitative analysis of aphid and primary parasitoid trophic relations of genus Alloxysta (Hymenoptera: Cynipoidea: Figitidae: Charipinae). Environmental Entomology 43 (6):14851495. https://doi.org/10.1603/EN14131

Ferrer-Suay M., Selfa J., Czyrnek M. \& Pujade-Villar J. 2015a. Charipinae Dalla Torre \& Kieffer, 1910 (Hymenoptera: Cynipoidea: Figitidae) from the Mercantour National Park (Alpes-Maritimes, France), with descriptions of three new species. Zoosystema 37 (1): 115-138. https://doi.org/10.5252/z2015n1a5

Ferrer-Suay M., Selfa J. \& Pujade-Villar J. 2015b. New contribution to the knowledge of the genus Alloxysta (Hymenoptera: Cynipoidea: Figitidae): revision of some type material. Annalen des naturhistorischen Museums in Wien, Serie B 117: 23-36.

Ferrer-Suay M., Selfa J. \& Pujade-Villar J. 2016. Revision of the Barbotin's Charipinae collection with description of a new Alloxysta species (Hymenoptera: Cynipoidea: Figitidae). Butlletí de la Institució catalana d'Història natural 80: 29-46.

Ferrer-Suay M., Selfa J., Seco-Fernández M.V. \& Pujade-Villar J. 2017. Review of the brachypterous species of Alloxysta Förster, 1869 (Hymenoptera: Cynipoidea: Figitidae: Charipinae) with a description of two new species. Turkish Journal of Zoology 41: 424-435.

Fitch A. 1861. Sixth report on the noxious and other insects of the state of New York. Transactions of New York State, Agricultural Society 20: 745-868. 
Förster A. 1869. Ueber die Gallwespen. Verhandllungen der kaiserlich-königlichen zoologishbotanischen Gesellschaft in Wien 19: 327-370.

Froggatt W.W. 1904. Experimental work with the peach aphis. Agricultural Gazette of New South Wales 15: 603-612.

Fülöp D., Melika G., Bechtold M. \& Bozsó M. 2010. Checklist of charipines of Hungary (Hymenoptera, Figitidae: Charipinae). Folia Entomologica Hungarica 71: 53-56.

Gaston K.J., Jones A.G., Hänel C. \& Chown S.L. 2003. Rates of species introduction to a remote oceanic island. Proceedings of the Royal Society of London. Series B - Biological Sciences 270: 1091-1098. https://doi.org/10.1098/rspb.2003.2332

Gautier D. 1921. Description de deux especes nouvelles, Trioxys placidus (Hym.: Braconidae) et Alloxysta gautieri J.-J. Kieffer (Hym.: Cynipidae). Bulletin de la Société entomologique de France 26: 302-307.

Giraud J. 1860. Enumeration des Figitides de l'Autriche. Verhandlungen der kaiserlich-königlichen zoologisch-botanischen Gesellschaft in Wien 10: 123-176.

Girault A.A. 1931. Hymenoptera, Thysanoptera; nova Australiensis. Privately published, Brisbane.

Girault A.A. 1932. New lower Hymenoptera from Australia and India. Privately published, Brisbane.

Girault A.A. 1933. Some Beauties Inhabitant not of Commercial Boudoirs but of Nature's Bosom, Notably New Insects. Privately published, Brisbane.

Guerra M., Fuentes-Contreras E. \& Niemeyer H.M. 1998. Differences in behavioral responses of Sitobion avenae (Hemiptera: Aphididae) to volatile compounds, following parasitism by Aphidius ervi (Hymenopteta: Braconidae). Écoscience 5 (3): 334-337.

Hartig T. 1840. Ueber die Familie der Gallwespen. Zeitschrift für Entomologie 2: 176-210.

Hartig T. 1841. Erster Nachtrag zur Naturgeschichte der Gallwespen. Zeitschrift für Entomologie 3: $322-358$.

Hedicke H. 1928. Beiträge zur Kenntnis der Cynipiden, (Hym.); XV: Neue und wenig bekannte Cynipiden aus dem unt Bemerkungen über eine andere Arten. Verhandlungen des Vereins für naturwissenschaftliche Unterhaltung 19: 72-96.

Hellén W. 1931. Zur Kenntnis der Cynipiden-fauna Islands. Göteborgs Kungliga Vetenskaps och Vitterhets Samhälles Handlingar 2 (5): 1-8.

Hellén W. 1963. Die Alloxystininen Finnlands (Hymenoptera: Cynipidae). Fauna Fennica 15: 1-23.

Hellén W. 1966. Cynipiden- and Proctotrupoidenfunde aus Norwegen (Hympenoptera). Norsk Entomologisk Tidsskrift 13: 393.

Hofsvang T. \& Hagvar E.B. 1983. Primary parasitoids (Hym., Aphidiidae) and hyperparasitoids on aphids from Norway. Fauna Norvegica, Serie B 30: 60-62.

Höller C., Borgemeister C., Haardt H. \& Powell W. 1993. The relationship between primary parasitoids and hyperparasitoids of cereal aphids - an analysis of field data. Journal of Animal Ecology 62: 12-21.

Horn D.J. 1988. Parasitism of cabbage aphid and green peach aphid (Homoptera: Aphididae) on collards in relation to weed management. Environmental Entomology 17 (2): 354-358.

Hübner G., Völkl W. \& Francke K.D. 2002. Mandibular gland secretions in alloxystine wasp (Hymenoptera, Cynipoidea, Charipidae): do ecological or phylogenetical constraints influence occurrence or composition? Biochemical Systematics and Ecology 20: 505-523.

https://doi.org/10.1016/S0305-1978(01)00137-5 
Ionescu M.A. 1959. Contributions to the study of parasitic Cynipides (Hymenoptera, Cynipoidea) of the rumian people's republic. Revue de Biologie (Academia Reuplicii Populare Rômine) 4 (2): 263-272.

Ionescu M.A. 1963. Contributions to the study of the superfamily Cynipoidea (Insecta-Hymenoptera) in the Romanian People's Republic. Revue de Biologie (Academia Republicii Populare Rômine) 8: 165176.

Ionescu M.A. 1969. Fauna Republicii Socialiste România. Insecta. Hymenoptera. Cynipoidea. Academia Republicii Socialiste România IX (6).

Kieffer J.J. 1900. Ueber Allotrinen. Wiener Entomologische Zeitung 19: 112-115.

Kieffer J.J. 1902a. Description de quelques Cynipides nouveaux ou peu connus et de deux de leurs parasites (Hymenopteres). Bulletin de la Société d'Histoire naturelle de Metz 10: 1-18.

Kieffer J.J. 1902b. Les Cynipides (part 2). $3^{\text {ième }}$ Tribu - Allotriines (Allotriinae). In: Andre E. (ed.) Species des Hyménoptères d'Europe et d'Algérie 7 (2): 5-78 + 592-602. Privately published, Beaune. https://doi.org/10.5962/bhl.title.10281

Kieffer J.J. 1904. Description de quelques Cynipides exotiques dont l'un forme un genre nouveau. Bulletin de la Société d'Histoire naturelle de Metz 2 (11): 59-66.

Kieffer J.J. 1909. Beschreibung neuer in Blattläusen schmarotzender Cynipiden. Naturwissenschaftliche Zeitschrift für Forst- und Landwirtschaft Stuttgart 7: 479-482.

Kierych E. 1979a. Notes on the genera Dilyta Förster, 1869 and Glyptoxysta Thomson, 1877. (Hymenoptera, Cynipoidea, Alloxystidae). Pt.1. Annales Zoologici 34 (15): 453-460.

Kierych E. 1979b. Galasówkowate Cynipoidea. Katalog Fauny Polski. Polska Akademia Nauk Instytut Zoologii 26 (2): 1-103.

Kierych E. 1988. A new genus and a new species of cynipoids (Hymenoptera, Cynipoidea, Charipidae) from Poland. Annales Zoologici 41: 351-354.

Krawczyk A., Hurej M. \& Jackowski J. 2009. Hyperparasitoids of aphids on maize in Opole region in Poland. Polish Journal of Entomology 78 (2): 161-168.

Lameere A. 1907. Manuel de la faune de Belgique; 3. Insectes supérieurs, Hyménoptères, Diptères, Lepidoptères. H. Lamertin, Brussels.

Lazzari S.N. 1985. Natural enemies dos afideos (Homoptera, Aphididae) da cevada (Hordeum sp.) no Paraná. Anais da Sociedade Entomológica do Brazil 14 (1): 5-15.

Lotfalizadeh H. 2002a. Natural enemies of cotton aphids in Moghan Region, Northwest of Iran. Proceedings of $15^{\text {th }}$ Iranian Plant Protection Congress, 7-11 Sep. 2002, Vol I. Pests: 36. Razi University of Kermanshah.

Lotfalizadeh H. 2002b. Parasitoids of Cabbage Aphid, Brevicoryne brassicae (L.) (Hom.: Aphididae) in Moghan Region. Agricultural Science 12 (1): 15-25

Lotfalizadeh H. \& van Veen F. 2004. Report of Alloxysta fuscicornis (Hym.: Cynipidae), a hyperparasitoid of aphids in Iran. Journal of the Entomological Society of Iran 23 (2): 119-120.

Mantero G. 1906. Materiali per un Catalogo degli Imenotteri Liguri. Parte IV. Cinipidi. Annali del Museo Civico di Storia Naturale di Genova 42: 445-467

Maria de Sousa B. \& Paes Bueno V.H. 1993-1994. Levantamento de predadores e parasitódes adultos associados a Brevicoryne brassicae (Linnaeus, 1758) (Homoptera: Aphididae), em culturas de couve. Boletín do International Cooperativa Biodiversidade Group 46: 23-34.

Marshall T.A. 1870. On some British Cynipidae. Entomologist's Monthly Magazine 6: 178-181. 
Menke A.S. \&. Evenhuis H.H. 1991. North American Charipidae: key to genera, nomenclature, species checklists, and a new species of Dilyta Förster (Hymenoptera: Cynipoidea). Proceedings of the Entomological Society of Washington 93: 136-158.

Mertins J.W. 1985. Hyperparasitoids from pea aphid mummies, Acyrtosiphon pisum (Homoptera: Aphididae), in North America. Annals of the Entomological Society of America 78 (2): 186-197.

Muesebeck C.F.W. \& Krombein K.V. 1951. Hymenoptera of America North of Mexico. Synoptic Catalog. Agriculture Monograph 2. U.S. Government Printing Office, Washington D.C.

Müller C.B., Adriaanse I.C.T., Belshaw R. \& Godfray H.C.J. 1999. The structure of an aphid-parasitoid community. Journal of Animal Ecology 68: 346-370. https://doi.org/10.1046/j.1365-2656.1999.00288.x

Nieves-Aldrey J.L., Fontal-Cazalla F.M., Garrido-Torres A.M. \& Rey del Castillo C. 2003. Inventario de Hymenoptera (Hexapoda) en el Ventorrillo: un rico enclave de biodiversidad en la Sierra de Guadarrama (España Central). Graellsia 59 (2-3): 25-43.

Oatman E.R., Trumble J.T. \& Voth V. 1983. Composition and relative abundance of parasites associated with aphid populations on strawberry in Southern California. Environmental Entomology 12 (6): 17141717.

O'Connor J.P. 2002. Alloxysta pedestris (Curtis) (Hymenoptera: Charipidae) new to County Dublin. Irish Naturalist's Journal 27 (3): 122-123.

O’Connor J.P. \& Nash R. 1997. A review of the Irish Charipidae (Hymenoptera) including nine species new to Ireland. Irish Naturalists' Journal 25: 410-411.

Pagliano G. 1995. Hymenoptera Cynipoidea. In: Minelli A., Ruffo S. \& La Posta S. (eds) Checklist delle specie della fauna italiana 96: 1-7. Calderini, Bologna.

Paretas-Martínez J. \& Pujade-Villar J. 2006. Two genera of Charipinae (Hymenoptera: Figitidae) from Australia: revision of the genus Thoreauana Girault, 1930 and description of Dilapothor n. gen. Australian Journal of Entomology 45: 219-226.

Paretas-Martínez J., Arnedo M.A., Melika G., Selfa J., Seco-Fernández M.V., Fülöp D. \& Pujade-Villar J. 2007a. Phylogeny of the parasitic wasp subfamily Charipinae (Hymenoptera, Cynipoidea, Figitidae). Zoologica Scripta 36: 153-172.

Paretas-Martínez J., Melika G. \& Pujade-Villar J. 2007b. Description of Lobopterocharips arreplegata gen.n. \& sp.n. (Hymenoptera: Figitidae: Charipinae) from Nepal, with notes on its phylogenetic position. Insect Systematics \& Evolution 38: 473-479.

Paretas-Martínez J., Ferrer-Suay M., Kovalev O., Melika G., Selfa J. \& Pujade-Villar J. 2011. Revision of the species of Dilyta Förster (Hymenoptera: Figitidae: Charipinae) present in the Holarctic, with description of four new species from the eastern Palaearctic. Zootaxa 2780: 29-38.

Peronti A.L.B.G., Fraga F.B., Rosa K.C.C., Teixeira M.T. \& Silva M.L. 2007. Efeitos da fragmentaçao florestal e da expansao agrícola sobre a comunidade de insetos fitófagos e himenópteros parasitoides no Parque Nacional da Serra dos Órgaos e arredores. In: Cronemberger C. \& Viveiros de Castro E.B. (eds) Ciência e Conservaçao na Serra dos Órgaos. Ibama, Brasília.

Prelipcean C., Mustata G. \& Prelipcean A. 2004. Natural control realized by parasitoid insects inside the Aphis fabae Scop. colonies. Analele stiintifice ale Universitatii "Al Jan. Cuza” Iasi, s. Biologie animal 50: 60-65.

Pujade-Villar J. 2005. Primera cita de Apocharips trapezoidea (Hartig, 1841) fuera del continente europeo (Hymenoptera: Cynipoidea: Figitidae: Charipinae: Charipini). Vieraea 33: 545. 
Pujade-Villar J. \& Paretas-Martínez J. 2006. Phaenoglyphis 'versus' Hemicrisis, and the description of a new sculptured species of Charipinae (Hymenoptera: Figitidae). European Journal of Entomology 103: 477-481.

Pujade-Villar J., Ferrer-Suay M.M., Selfa J., Seco-Fernández M.L., Kovalev O., Melika G. \& ParetasMartínez J. 2010a. On Bothrioxysta colorata Belizin, 1962 (Figitidae: Charipinae) and some other Charipinae species described by Belizin. Entomofauna, Zeitschrift für Entomologie 31 (18): 285-292.

Pujade-Villar J., Ferrer-Suay M.M., Selfa J. \& Caicedo-Ramírez G. 2010b. Primera cita de Chariinae (Hym., Figitidae) para Colombia. Boletín del Museo de Entomología de la Universidad del Valle 11 (2): $28-32$.

Pujade-Villar J., Ros-Farré P., Durán S. \& Ventura D. 2001. Cynipoideus col.lectats a Menorca (Hymenoptera). Sessió d'Entomologia de la Institució Catalana d'Història Natural-Societat Catalana de Lepidopterologia 11: 81-86.

Available from http://www.raco.cat/index.php/SessioEnto/article/view/192159/258069 [accessed 21 Mar. 2018]

Pujade-Villar J., Díaz N., Evenhuis H.H. \& Ros-Farré P. 2002. South American Charipinae: Review and description of two new species (Hymenoptera: Cynipoidea: Figitidae). Annals of the Entomological Society of America 95 (5): 541-546.

Pujade-Villar J., Paretas-Martínez J., Selfa J., Secó-Fernández M.V., Fülop D. \& Melika G. 2007. Phaenoglyphis villosa (Hartig 1841) (Hymenoptera: Figitidae: Charipinae): a complex of species or a single but very variable species? Annales de la Société entomologique de France 43 (2): 169-179. https://doi.org/10.1080/00379271.2007.10697508

Quinlan J. 1974. The British Cynipoidea (Hymenoptera) described by P. Cameron. Bulletin of the Natural History Museum, Entomology Series 31: 1-21.

Quinlan J. \& Fergusson N.D.M. 1981. The status and identity of the Cynipoidea (Hymenoptera) described by J. Curtis. Entomological Gazette 32: 251-256.

Rakhshani E., Talebi A.A. \& Sadeghi E. 2001. The first report of aphid hyperparasitoid, Alloxysta (Alloxysta) citripes (Thomson) (Hymenoptera: Cynipidae) from Iran. Applied Entomology and Phytopathology 69 (1): 184-185.

Rakhshani E., Talebi A.A., Sadeghi E., Kavallieratos N.G. \& Rashed A. 2004. Seasonal parasitism and hyperparasitism of walnut aphid, Chromaphis juglandicola (Kaltenbach) (Hom., Aphididae) in Tehran province. Journal of the Entomological Society of Iran 23 (2): 1-11.

Rohwer S.A. \& Fagan M. 1917. The type-species of the genera of the Cynipoidea, or the gall wasps and parasitic Cynipoids. Proceedings of the United States National Museum 53: 357-380.

Rohwer S.A. \& Fagan M. 1919. Additions and corrections to "The type-species of the genera of the Cynipoidea, or the gall wasps and parasitic Cynipoids". Proceedings of the United States National Museum 55: 337-340.

Rondani C. 1878. Osservazioni sopra parecchie specie de esapodi Afidicidi e sui loro nemici. Nuovi Annali delle Scienze Naturali e Rendiconto dei Lavori dell' Accademia delle Scienze dell'Instituto Di Bologna 9: 5-33.

Ronquist F. 1999. Phylogeny, classification and evolution of the Cynipoidea. Zoologica Scripta 28: 139-164.

Sampaio M.V., Korndörfer A.P., Pujade-Villar J., Hubaide J.E.A., Ferreira S.E., Arantes S.O., Bortoletto D.M., Guimaraes C.M., Sánchez-Espigares J.A. \& Caballero-López B. 2017. Brassica aphid (Hemiptera: Aphididae) populations are conditioned by climatic variables and parasitism level: 
a study case of Triângulo Mineiro, Brazil. Bulletin of Entomological Research, 107 (3): 410-418. https://doi.org/10.1017/S0007485317000220

Sanders D. \& van Veen F.J.F. 2010. The impact of an ant-aphid mutualism on the functional composition of the secondary parasitoid community. Ecological Entomology 35 (6): 704-710.

https://doi.org/10.1111/j.1365-2311.2010.01230.x

Silvestri F. 1915. Contributo all a conoscenza degli insetti dell'olivo del'Eritrea e dell'Africa meridionale. Bolletino del Laboratorio di Zoologia Generale e Agraria della Facoltà Agraria in Bytici 9: 240-234.

Spencer H. 1926. Biology of the parasites and hyperparasites of Aphis. Annals of the Entomological Society of America 19: 119-157.

Suay V.A., Luna F. \& Michelena J.M. 1998. Parasitoids not aphidiins [sic] of aphids (Chalcidoidea: Aphelinidae) and hyperparasitoids of the superfamilies Chalcidoidea, Ceraphronoidea and Cynipoidea (Hymenoptera: Apocrita: Parasitica) in the province of Valencia. Boletín de la Asociación española de Entomologia 22 (1-2): 99-113.

Sullivan D.J. 1987. Insect hyperparasitism. Annual Review of Entomology 32: 49- 70.

Sullivan D.J. \& van den Bosch R. 1971. Field ecology of the primary parasites and hyperparasites of the potato aphid, Macrosiphum euphorbiae, in the east San Francisco Bay Area. Annals of the Entomological Society of America 64 (2): 389-394.

Sullivan D.J. \& Völk1 W. 1999. Hyperparasitism: Multitrophic ecology and behavior. Annual Review of Entomology 44:291-315.

Takada H. \& Nakamura T. 2010. Native primary parasitoids and hyperparasitoids attacking an invasive aphid Uroleucon nigrotuberculatum in Japan. Entomological Science 13: 269-272.

Taschenberg E.L. 1866. Die Hymenoptera Deutschlands. E. Kummer, Liepzig.

Teixeira M. 1991. Estudo das interaçoes "plantalafidea/parasitoide e hiperparasitóide" em ambientes naturais e antrópicos. Centro de Ciéncias Biológicas e da Saúde, Programa de Pós-Graduaçao em Ecologia e Recursos Naturais, Universidade Federal de Sao Carlos.

Thomson C.G. 1862. Forsok till uppstallning och beskrifning af Sveriges Figiter. Öfversigt af Kongliga Svenska Vetenskaps-akademiens förhandlingar 18: 395-420.

Thomson C.G. 1877. Ofversikt af Sveriges Cynips-arter. Opuscula Entomologica 8: 778-820.

Tizado E.J. \& Nuñez-Pérez E. 1993. Some data on Alloxistinae (Hym., Charipidae) in Spain. Aphidophaga 5-International Organization of Biological Control Symposium 1993: 97. The International Organization for Biological Control, Zürich.

Torras-Casals C. 1996. Especies de Alloxystinae (Hymenoptera, Cynipoidea, Charipinae), hiperparásitos de áfidos en la provincia de Barcelona. Real Sociedad Española de Historia Natural (vol. extraord. XII Bienal, Madrid): 196-197.

Trandafirescu M., Trandafirescu I., Gavat C. \& Spita V. 2004. Entomophagus complexes of some pests in apple peach orchards in Southeastern Romania. Journal of Fruit and Ornamental Plant Research 12: 253-261.

Valentine E.W. 1975. Additions and corrections to Hymenoptera on aphids in New Zealand. The New Zealand Entomologist 6 (1): 59-61.

Van Veen F.J., Belshaw R. \& Godfray H.C.J. 2003. The value of the ITS2 region for the identification of species boundaries between Alloxysta hyperparasitoids (Hymenoptera: Charipidae) of aphids. European Journal of Entomology 100: 449-453. https://doi.org/10.14411/eje.2003.067 
Vasileva-Sumnalieva L. 1976. On the fauna and biology of the subfamily Charipinae (Hymenoptera, Cynipidae) in Bulgaria. Acta Zoologica Bulgarica 5: 22-26.

Vaz L.A.L., Tavares M.T. \& Lomônaco C. 2004. Diversidade e tamanho de himenópteros parasitóides de Brevicoryne brassicae L. e Aphis nerii Boyer de Fonscolombe (Hemiptera: Aphididae). Neotropical Entomology 30 (2): 225-230. https://doi.org/10.1590/S1519-566X2004000200013

Watanabe C. 1950. Charipidae of Japan. Insecta Matsumurana 17 (2): 83-89.

Weld L.H. 1920. A new parasitic Cynipid reared from a clover aphid (Hym.). Entomological News 31: $14-16$.

Weld L.H. 1952. Cynipoidea (Hym.). 1905-1950. Privately published, Ann Arbor, Michigan.

West S.A., Cook J.M., Werren J.H. \& Godfray H.C.J. 1998. Wolbachia in two insect host-parasitoid communities. Molecular Ecology 7: 1457-1465. https://doi.org/10.1046/j.1365-294x.1998.00467.x

Westrum K., Klingen I., Hofsvang T. \& Hågvar E.B. 2010. Checklist of primary parasitoids and hyperparasitoids (Hymenoptera, Apocrita) on aphids (Hemiptera, Aphididae) from Norway. Norwegian Journal of Entomology 57: 142-153.

Westwood J.O. 1833. Notice of the habits of a Cynipidous insect parasitic upon the Aphis rosae with descriptions of several other parasitic Hymenoptera. Magazine of Natural History 6: 491-497. Available from http://www.biodiversitylibrary.org/item/19636\#page/505/mode/1up [accessed 2 Feb. 2018].

Wilson C.G. \& Swincer D.E. 1984. Hyperparasitism of Therioaphis trifolii f. maculate (Homoptera: Aphididae) in South Australia. Journal of the Australian Entomological Society 23 (1): 47-50.

Zetterstedt J.W. 1838. Insecta Lapponica descripta: Hymenoptera: 315-476. Voss, Leipzig.

Zuparko R.L. \& Dahlsten D.L. 1995. Parasitoid complex of Eucallipterus tiliae (Homoptera: Drepanosiphidae) in northern California. Environmental Entomology 24 (3): 730-737. https://doi.org/10.1093/ee/24.3.730

Manuscript received: 3 April 2017

Manuscript accepted: 7 August 2017

Published on: 19 April 2018

Topic editor: Gavin Broad

Desk editor: Kristiaan Hoedemakers

Printed versions of all papers are also deposited in the libraries of the institutes that are members of the EJT consortium: Muséum national d'Histoire naturelle, Paris, France; Botanic Garden Meise, Belgium; Royal Museum for Central Africa, Tervuren, Belgium; Natural History Museum, London, United Kingdom; Royal Belgian Institute of Natural Sciences, Brussels, Belgium; Natural History Museum of Denmark, Copenhagen, Denmark; Naturalis Biodiversity Center, Leiden, the Netherlands; Museo Nacional de Ciencias Naturales-CSIC, Madrid, Spain; Real Jardín Botánico de Madrid CSIC, Spain. 